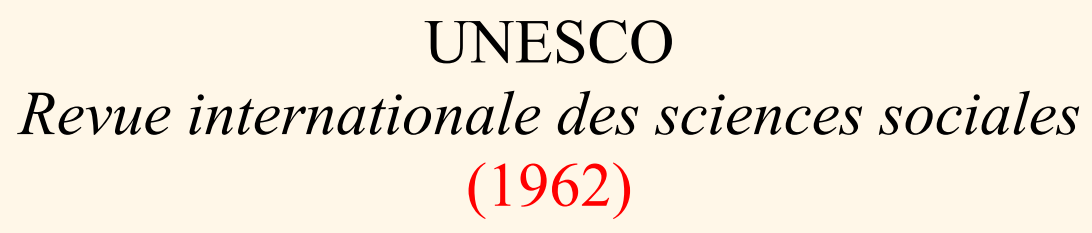

\title{
Images de la femme dans la société
}

Un document produit en version numérique par Gemma Paquet, bénévole, professeure à la retraite du Cégep de Chicoutimi Courriel:mgpaquet@videotron.ca

dans le cadre de la collection: "Les classiques des sciences sociales" fondée dirigée par Jean-Marie Tremblay, professeur de sociologie au Cégep de Chicoutimi

Site web: http://www.uqac.uquebec.ca/zone30/Classiques_des_sciences_sociales/index.html

Une collection développée en collaboration avec la Bibliothèque

Paul-Émile-Boulet de l'Université du Québec à Chicoutimi

Site web: http://bibliotheque.uqac.uquebec.ca/index.htm 
Cette édition électronique a été réalisée par Gemma Paquet, bénévole, professeure à la retraite du Cégep de Chicoutimi à partir de :

\section{Images de la femme dans la société}

Un dossier publié dans la Revue internationale des sciences sociales, revue trimestrielle, vol. XIV, no 1, 1962 (pp. 3 à 178). Paris : UNESCO, 1962.

Remarque : Nous avons communiqué à plusieurs reprises avec la direction de la Revue internationale des sciences sociales afin d'obtenir une confirmation écrite d'autorisation de diffuser ce beau dossier comparatif sur les femmes, en vain.

Polices de caractères utilisée :

Pour le texte: Times, 12 points.

Pour les citations : Times 10 points.

Pour les notes de bas de page : Times, 10 points.

Édition électronique réalisée avec le traitement de textes Microsoft Word 2001 pour Macintosh.

Mise en page sur papier format

LETTRE (US letter), 8.5' x 11',)

Édition complétée le 16 septembre 2004 à Chicoutimi, Québec.

\section{Fait avec}

Macintosh 


\section{Images de la femme dans la société}

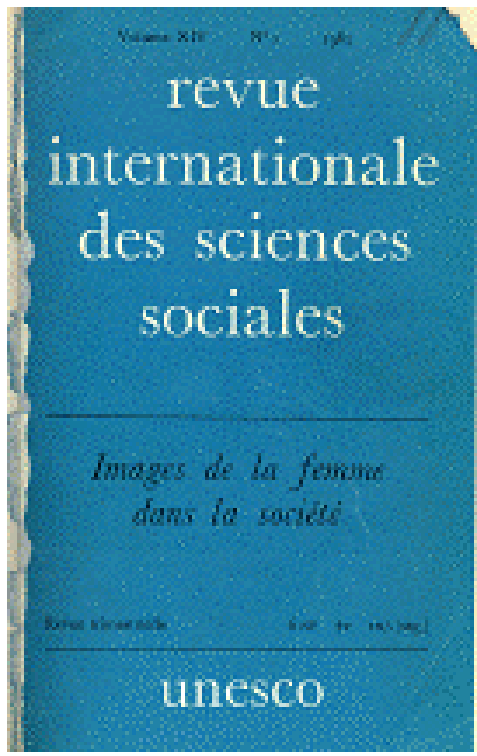

Un dossier publié dans la Revue internationale des sciences sociales, revue trimestrielle, vol. XIV, no 1, 1962 (pp. 3 à 178).

Paris : UNESCO, 1962. 


\section{Table des matières}

\section{Images de la femme dans la société}

"Introduction", par P. Chombart de Lauwe, [L'auteur travaille à l'École pratique des hautes études, Groupe d'ethnologie sociale, Paris.]

I. M. J. Chombart de Lauwe, "Image de la situation de la femme dans la société urbaine française" [M. J. Chombart de Lauwe, Groupe d'ethnologie sociale, CNRS, Paris.]

II. Les attitudes à l'égard de la condition de la femme dans les familles ouvrières polonaises

1. Antonina Kloskowska, "Généralités. Attitudes à l'égard des rôles respectifs et de l'égalité des deux sexes et à l'égard des enfants" [L'auteur travaille à l'Université de Lodz.]

2. J. Piotrowski, "Attitudes à l'égard du travail des femmes" [L'auteur travail à l'Université de Varsovie.]

III. Femmes et professions au Maroc

1. Nelly Forget, "Attitudes à l'égard du travail professionnel de la femme au Maroc" [L'auteure travaille avec le Groupe d'ethnologie sociale, Paris.]

2. Khadidja Nouacer, "Évolution et travail professionnel de la femme au Maroc" [L'auteure travaille comme assistante sociale à Rabat.]

IV. Trois études préliminaires : Canada, Côte-d'Ivoire, Togo, par Guy Rocher, R. Clignet et F. N'Sougan Agblemagnon

1. Guy Rocher, "Les modèles et le statut de la femme canadienne-française" [l'auteur est sociologue et professeur de sociologie à l'Université de Montréal]

2. R. Clignet, "Les attitudes de la société à l'égard des femmes en Côte d'Ivoire" [L'auteur travaille à l'Institut de statistiques, Abidjan.]

3. F. N'Sougan Agblemagnon, "Recherche sur les attitudes vis-à-vis de la femme togolaise" [L'auteur travaille au Centre d'études sociologiques, Paris et Rome.]

V. Leopold Rosenmayr, "La femme autrichienne, son rôle et l'image qu'on s'en fait" [L'auteur travaille à l'Université de Vienne.]

VI. Olivera Buric, "Les attitudes à l'égard de la condition de la femme en Yougoslavie" [L'auteur travaille à l'Institut des sciences sociales de Belgrade] 
Images de la femme dans la société

\section{Introduction}

\section{P. Chombart de Lauwe}

Retour à la table des matières du livre

Table des matières de l'introduction

1. Le problème : l'image de la femme dans la société comme moteur ou frein du changement des structures sociales

2. L'enquête internationale : principes, méthode et organisation

3. Deux images dynamiques: la femme au travail et l'égalité des sexes

a) L'image de la femme au travail

b) La question de l'égalité de la femme et de l'homme dans la famille et dans la société

4. Problèmes généraux d'évolution et perspectives

a) Traditions culturelles et universalisme

b) Oppositions entre les images dynamiques et les comportements

c) La perception des situations et la perception de l'évolution

5. La nouvelle image de la femme dans la société à venir

a) La nouvelle image de la femme et l'avenir du couple humain

b) La nécessaire transformation des structures sociales

c) Développements ultérieurs des recherches 
Retour à la table des matières de l'introduction

Jamais le statut de la femme dans la société n'a posé plus de questions à l'échelle mondiale qu'il n'en pose aujourd'hui. jamais il n'est apparu plus clairement que l'évolution des structures sociales y était liée. Les bouleversements qui se sont opérés ou qui s'opèrent dans les pays socialistes et dans les sociétés en cours de développement, de la Chine à l'Afrique, de l'URSS à l'Amérique du Sud, ne sont pas les seules raisons des secousses qui agitent la vieille Europe ou l'Amérique du Nord. À l'Est comme à l'Ouest, les déclarations de principe, les discussions passionnées qui ont lieu dans la vie politique, les mouvements d'opinion, la presse sont autant de preuves de l'ampleur et de l'importance du problème.

De nombreuses études sociologiques ont déjà mis en relief divers aspects de l'évolution du statut de la femme. Le travail professionnel, la participation de la vie politique, le rôle social, le rôle familial, etc., ont donné lieu à la publication d'ouvrages et d'articles divers dans beaucoup de pays. Nous ne songeons à en faire ici ni un tableau complet, ni la synthèse. L'enquête internationale que nous avons entreprise apportera, nous l'espérons, à ces recherches un élément nouveau, mais elle se situe différemment. Ce n'est pas la femme dans la société et l'évolution de sa situation que nous étudions, mais l'image que s'en font les hommes et les femmes dans diverses régions du monde, en fonction de leur culture, des transformations industrielles qui s'opèrent, des courants de pensée nouveaux qui apparaissent, de la place qu'ils occupent eux-mêmes dans la société, de leur expérience personnelle. Nous essayons de comprendre comment ils perçoivent et se représentent la femme dans l'ensemble des structures sociales et comment ils la voient changer. Les images qui naissent dans ces conditions et leurs modifications peuvent soit freiner, soit accélérer les changements de statut réel et, dans certains cas, les provoquer, si elles correspondent aux aspirations profondes des peuples.

Dans ces conditions, si le changement de statut de la femme est une des pièces maîtresses de la transformation des structures sociales et si l'image qu'on se fait de ce changement joue un rôle si considérable, le but de notre entreprise apparaît plus clairement. À travers la représentation et la perception, les images et les modèles, les aspirations et les valeurs qui y sont liées, c'est toute la possibilité d'évolution d'une société que nous envisageons. Dans une enquête internationale, c'est l'harmonisation de cette évolution dans les différents pays, en tenant compte de l'apport original de chacun d'eux, que nous voudrions commencer à étudier.

Telle que nous pouvons la décrire actuellement, l'enquête donne une idée encore incomplète des possibilités ouvertes par cette voie de recherche. Pourtant, avant de franchir de nouvelles étapes, nous avons pensé qu'une première confrontation avec un large public était nécessaire. Sur les buts de l'enquête et le problème posé, sur la méthode et l'organisation du travail, sur les premiers résultats et les perspectives ultérieures, des discussions utiles pour l'orientation des recherches internationales peuvent déjà avoir lieu. 


\section{Le problème : l'image de la femme dans la société comme moteur ou frein du changement des structures sociales}

$\underline{\text { Retour à la table des matières de l'introduction }}$

Une question de définition se pose tout d'abord. Étudier comment les hommes et les femmes se représentent la femme dans l'ensemble des structures sociales suppose au préalable l'étude de la manière dont ils perçoivent les faits, les situations, les personnages qui vont s'ordonner dans cette représentation. Souvent, les comportements sociaux et les attitudes des sujets sont orientés suivant une série de perceptions, sans que les intéressés les relient encore très clairement les unes aux autres. Ils se réfèrent à deux catégories d'images, les unes aux contours plus nets, ayant une structure mieux définie, qui correspondent réellement à des représentations - au sens que Durkheim et d'autres auteurs ont donné à ce mot - les autres plus floues, plus mouvantes, qui sont soumises aux fluctuations des perceptions successives.

La perception doit elle-même ici être entendue dans le sens large d'appréhension, à un moment donné, d'un ensemble d'éléments du milieu social tenant à l'expérience des sujets dans une culture donnée. Nous avons insisté sur les différences de perception des objets, des situations et d'autrui suivant les classes sociales à l'intérieur d'un même pays. À plus forte raison, devons-nous tenir compte des écarts bien plus grands qui existent entre les civilisations différentes. Nous rejoignons, dans cette perspective, une longue série de travaux sur la perception sociale, publiés dans divers pays, que nous ne pouvons pas examiner dans une étude aussi brève.

Suivant leurs perceptions, les individus ont une conscience plus ou moins claire des groupes auxquels ils appartiennent, de l'ensemble des structures sociales, de leur situation dans cet ensemble, et les images qui apparaissent à leur esprit s'y relient plus ou moins nettement. Les individus d'un même groupe, d'un même milieu social, d'une même catégorie, dans une même société, ont, en général, des images identiques, plus ou moins proches d'une représentation bien définie ou d'une simple perception souvent diffuse.

Ces images que nous saisissons ainsi se rapportent à des modèles (patterns) hérités de la tradition, importés d'autres cultures ou élaborés récemment dans un contexte nouveau, mais elles ont un contenu collectif et une force active qui varient suivant les milieux sociaux, les moments et les personnalités. Ainsi, l'image que se fait un ouvrier français de la femme dans la famille se rapporte à un modèle traditionnel de la femme-épouse et de la femme-mère, et à un modèle plus récent, relatif au travail professionnel de la femme, à sa participation à la vie politique. Cette image 
éveille, en se formant, des sentiments parfois contradictoires, qui poussent l'ouvrier à agir de telle ou telle manière avec sa propre femme, avec ses enfants ou avec des camarades de travail. Elle aura une influence sur ses prises de position politiques ou syndicales, et pourra l'amener à modifier son échelle de valeurs ou sa vision de l'ensemble de la société. Dans ce sens, le mot «image » ou l'expression «image guide » (Leitbild en allemand) nous paraît mieux s'adapter que l'expression « modèle culturel » (pattern of culture). Nous verrons plus loin les distinctions à introduire à ce sujet.

L'image du statut de la femme, définie de cette manière, a une grande importance dans tous les pays, mais nous avons cru nécessaire, dans une première phase de recherche, de nous limiter à un nombre restreint de sociétés représentant des types d'évolution particulièrement significatifs à l'heure actuelle - Pologne, Canada, France, Maroc, Afrique noire. Chacune de ces sociétés et les images qui leur correspondent seront décrites plus longuement dans les différents articles, mais il est nécessaire d'en dire dès maintenant quelques mots.

La Pologne, pays socialiste, offre une évolution brusque, provoquée à la fois par la guerre, le changement de régime et les transformations techniques. Alors que la vie rurale y dominait très largement il y a trente ans, le pourcentage de la population urbaine atteint maintenant près de la moitié de la population totale [A. Kloskowska]. Le pourcentage des femmes mariées exerçant un travail professionnel a doublé pour l'ensemble du pays. Mais, malgré les changements profonds, certaines traditions demeurent fortes. Les raisons qui poussent les ouvrières à travailler sont surtout, nous le verrons, des raisons d'ordre financier [Piotrowski].

De forte tradition rurale également, le Canada de langue française s'oppose à la Pologne par un niveau de vie beaucoup plus élevé et par un régime politique conservateur jusqu'à une période très récente. L'industrialisation et le développement économique y sont particulièrement poussés, mais le taux de participation des femmes mariées à la vie professionnelle y est très faible. Les images de la famille et de la parenté y ont gardé une force attractive exceptionnelle [G. Rocher].

La France se trouve dans une situation intermédiaire. Les traditions familiales y sont restées relativement fortes, mais les courants idéologiques contradictoires, les chocs des guerres successives et la progression économique de ces dernières années ont tendu à modifier rapidement la situation. Dans la région parisienne, où s'est effectuée l'enquête, le pourcentage des femmes mariées avec enfant, ayant un travail professionnel, est relativement élevé [M. J. Chombart de Lauwe].

Cette situation intermédiaire apparaît également, mais d'une manière différente, en Autriche [L. Rosemayr].

Les rapports entre l'industrialisation et l'évolution de la situation de la femme y sont liés à la fois à l'évolution urbaine de Vienne et à la place particulière du pays entre l'Europe de l'Est et l'Europe de l'Ouest. Bien que sa voisine géographique, la Yougoslavie offre un autre exemple, du fait de l'expérience socialiste qui s'y poursuit [O. Burié]. Les oppositions entre les images traditionnelles d'un passé récent et les modèles nouveaux y sont d'autant plus instructives.

Le Maroc se caractérise par un type de civilisation très différent. Économiquement, il s'agit d'un pays en voie d'industrialisation ayant un niveau de vie encore très 
bas par rapport aux précédents, bien que la situation y soit moins défavorable que dans de nombreux autres pays africains. La très grande majorité de la population est restée rurale, et le nombre des femmes exerçant un travail professionnel est encore très faible. L'existence de la polygamie, les traditions familiales très vivaces, le port du voile, la conception du mariage ont marqué profondément le statut de la femme. Mais la participation à la résistance et à la libération du pays lui ont donné brusquement d'immenses possibilités. Aussi les changements sont-ils bien plus profonds et bien plus rapides que dans les pays européens. Les images auxquelles se réfèrent les sujets sont à la fois plus complexes et plus dynamiques.

Ces oppositions sont plus fortes encore en Afrique noire, où la situation coloniale et les modèles traditionnels ont joué, chacun dans leur sens, des rôles particulièrement importants. Il doit s'y ajouter des différences culturelles très considérables à l'intérieur d'un même pays, la persistance des systèmes complexes de parenté même en milieu urbain, et le fait que les coutumes liées à la dot ont donné à la femme un rôle d'échange particulier [R.Clignet]. Cependant, dans certaines régions, la tradition laissait aux femmes une sorte de pouvoir dissimulé, dont le rôle est plus considérable qu'on ne pensait. Les nouvelles organisations féminines qui se constituent peuvent s'appuyer parfois sur ces traditions [N'Sougan Agblemagnon].

Pour mieux situer les problèmes féminins propres à ces différents terrains d'observation dans le contexte international actuel, il faudrait faire appel à toutes les recherches portant sur l'évolution du statut de la femme. L'ouvrage publié sous la direction de Denise Schieffner sur la femme africaine et plusieurs travaux collectifs sur l'industrialisation et l'urbanisation au sud du Sahara, des articles de la revue Présence africaine, etc., nous permettront, par exemple, de suivre différentes étapes des changements et de mieux définir le rôle des images guides que nous voulons étudier. Dans les pays industrialisés depuis longtemps, des travaux comme ceux de A. Myrdal (Suède), V. Klein (Angleterre), A. Pizzorno (Italie), J. Stoetzel et A. Girard, P. Fougeyrollas, M. Guilbert et V. Isambert-Jamati, A. Michel, etc., sur la famille, les rôles masculins et féminins, la vie professionnelle féminine nous aideront à cerner les rôles économiques et affectifs. Parmi les enquêtes en cours, celles de A. Pizzorno et d'autres chercheurs en Italie, la nouvelle recherche de V. Isambert en France sur les attitudes des parents à l'égard de l'éducation des filles, celle de O. Miret en Tunisie, nous apporteront souvent une aide encore plus directe, étant donné les recoupements des préoccupations.

Les nouveaux codes nous offrent une occasion de réflexion d'un autre ordre. Le code polonais, qui remplace l'expression puissance «paternelle » par celle de puissance «parentale », ou le code marocain, qui prévoit une protection de la femme en tenant compte des traditions religieuses propres au pays, nous font saisir directement la transformation des institutions liée aux images nouvelles. D'un autre côté, les discussions politiques sur le vote des femmes, les congrès internationaux sur ce sujet, les assemblées féminines dans les pays africains et asiatiques, les réunions de femmes chefs d'entreprise, les travaux des associations familiales devront faire l'objet d'analyses plus approfondies dans la perspective de notre recherche actuelle.

Il semble que, dans les pays où les femmes ont connu plus particulièrement une situation de dépendance, les réactions soient les plus violentes et les mesures institutionnelles les plus radicales. La loi chinoise de 1950 sur le mariage et les diverses décisions qui ont réglé le statut de la femme dans ce pays vont plus loin, en matière de principes, que ce n'est le cas dans tous les autres pays. Mais cette exigence n'est 
pas liée seulement à la doctrine communiste. Dans des pays musulmans où les femmes ont participé aux maquis ou à la révolution, leurs revendications, dans un tout autre contexte, ne sont pas moins vigoureuses.

Il ne faut pas s'étonner de voir, sur tous les plans, un déchaînement de passion lorsqu'on aborde le sujet du rôle de la femme et de son statut dans la société. D'un côté, trop d'hommes (et de femmes) sont attachés, sans s'en rendre entièrement compte, aux modèles anciens et croient que toute conception de la famille est impossible hors de ces modèles. De l'autre, trop de femmes ont souffert au plus intime de leur être de l'injustice et de l'incompréhension de leurs partenaires et des législateurs. Nos sociétés ont vécu longtemps, nous l'avons dit ailleurs, accrochées au mythe de là «virilité », et à l'image d'une supériorité biologique, psychologique, juridique, spirituelle de l'homme. A la limite, elles ont abouti aux caricatures bourgeoise, fasciste, ou nazie du père protecteur, du chef prédestiné, du héros guerrier de race supérieure. Il fallait bien alors trouver dans la «féminité » la contrepartie de soumission, de douceur, d'abnégation.

On conçoit facilement la violence des réactions et les aberrations auxquelles arrivent certaines féministes, dont on comprend facilement les blessures. Un peu partout naissent, pour répondre au mythe précédent, ce que nous avons appelé des contre-mythes de la virilité ${ }^{1}$. Ceux - ou plutôt celles -qui s'y attachent ont alors tendance à confondre égalité des sexes et similitude, ce qui n'a plus aucun sens. Cependant, chez les législateurs les plus révolutionnaires mais les plus lucides, les différences entre l'homme et la femme restent très marquées. Les codes des Pays communistes prévoient des mesures de protection de la femme au moment de la maternité, de l'éducation des enfants en bas âge, voire des périodes de menstruation. La véritable égalité des sexes consiste à créer des structures sociales et des institutions telles que les femmes puissent jouir pleinement des mêmes droits que les hommes tout en restant elles-mêmes.

L'image de cette femme libérée, égale de l'homme bien que différente, est précisément celle que nous voyons apparaître, sous des formes diverses, dans les pays étudiés. Suivant ce qu'elle sera, suivant sa puissance d'attraction, la société tout entière prendra tel ou tel visage. La vie politique, l'entreprise, l'organisation de l'espace résidentiel, les institutions éducatives seront fatalement orientées en fonction de cette image. Le planificateur aurait tort d'oublier, dans ses prévisions, ce facteur essentiel qui peut un jour renverser ses calculs les plus savants. Ce n'est pas vers le nez de Cléopâtre que se tournent aujourd'hui les historiens des temps modernes, mais vers la femme, dans sa personnalité tout entière, dans sa liberté conquise, qui, finalement, à sa manière, par les préoccupations qu'elle impose, préside aux destinées du monde.

1 «Les mythes de la virilité et la guerre des sexes ». Article à paraître dans un ouvrage collectif. 


\section{L'enquête internationale : principes, méthode et organisation}

Retour à la table des matières de l'introduction

Entreprendre une enquête internationale sur un sujet aussi brûlant pouvait paraître présenter des difficultés insurmontables. En fait, les problèmes qu'elle pose ne sont pas différents de ceux que posent les autres entreprises du même genre. Ce n'est pas le sujet, mais le principe même des enquêtes comparatives à l'échelle mondiale que nous voudrions d'abord mettre en cause.

Si de telles enquêtes n'ont pas, jusqu'ici, pris plus d'ampleur, c'est, il faut bien le dire, qu'une méthode acceptable n'a pas encore été trouvée. Nous avons assisté à de nombreux congrès, au cours desquels des projets ont été élaborés, mais la proportion des travaux qui sont effectivement réalisés ensuite reste en général très limitée. Cela ne tient pas seulement aux difficultés matérielles de financement et d'organisation, mais beaucoup plus, croyons-nous, aux hésitations des participants devant les problèmes d'orientation.

Dans le cas présent, nous avons procédé à une élaboration par étapes successives, qui ne prétend nullement résoudre tous les problèmes, mais peut susciter quelques réflexions. Les principes de départ peuvent être résumés en quelques mots :

1. À partir d'une proposition fondée sur des expériences faites dans un ou plusieurs pays, préparer, avec trois ou quatre chercheurs, des hypothèses de travail, un premier plan de questionnaire, des éléments de guides d'interviews, un avantprojet d'échantillonnage dans un nombre très restreint de pays.

2. Prévoir des approches différentes - ethnographiques, sociologiques et psychologiques - en tenant compte du contexte historique, de la conjoncture économique, du développement technique, des traditions culturelles et des courants idéologiques.

3. Demander le plus possible aux représentants de chaque pays de procéder aux enquêtes à l'intérieur de leur propre culture, pour éviter les préjugés du visiteur, qui sont encore plus graves que ceux des sujets eux-mêmes. Nous pensons que,, trop souvent, les enquêtes rapides faites par un chercheur dans une société différente de la sienne fournissent des renseignements plus sûrs sur sa propre mentalité et sur sa façon de percevoir un autre peuple qu'elles ne donnent une image exacte de ce peuple. Malheureusement, dans de nombreux cas, en particulier dans les pays en cours de développement, ce souhait est irréalisable. Il faut 
alors que les chercheurs étrangers travaillent de plus en plus en collaboration avec des représentants du pays qui prennent des responsabilités dans l'enquête.

4. L'utilisation d'un questionnaire passe-partout, même élaboré en commun par les chercheurs des différents pays, est une illusion. Le nombre des questions qui peuvent avoir le même sens dans plusieurs contextes culturels différents est forcément très limité. Ce ne sont pas toujours les questions les plus intéressantes. Les questionnaires et les guides d'interviews doivent être repensés sur le terrain, au cours des premières phases d'observation libre. De toute façon, dans la perspective interdisciplinaire dont nous avons parlé, les études pour questionnaires ne doivent pas nécessairement tenir la première place dans la recherche.

Ces principes n'ont été mis en application que partiellement dans la première série d'enquêtes que nous présentons. Mais l'historique de la préparation montrera comment nous avons cherché à nous en approcher autant que possible. Essayons d'en suivre les diverses phases.

Première phase. Les recherches que nous avions entreprises en France sur la perception et la représentation des structures sociales n'avaient donné lieu qu'à des enquêtes limitées à l'intérieur d'une seule culture. En liaison avec les travaux que nous avions commencés d'autre part, sur l'évolution de la famille, l'étude de l'image de la situation de la femme dans la société nous est apparue, en 1957, comme une occasion excellente d'enquête comparative internationale. C'est pourquoi nous l'avons soumise à un groupe de chercheurs de plusieurs pays, lors d'une première discussion dans une rencontre internationale, à laquelle participaient A. Kloskowska (Pologne), Ishwaran (Inde), N'Sougan Agblemagnon (Togo), L. Rosenmayr (Autriche). Le thème proposé fut accepté et les participants s'engagèrent à entreprendre, dans leurs pays respectifs, des enquêtes pilotes sur les attitudes des hommes de milieux ouvriers à l'égard de la condition de la. femme dans la société.

Deuxième phase. Cependant, ce premier projet ne prit réellement corps, sous une forme différente, que lors d'un séjour de plusieurs mois que A. Kloskowska et $\mathrm{G}$. Rocher (Canada) firent à Paris et au cours duquel un premier projet de questionnaire fut élaboré. Nous voulions débuter avec trois pays seulement, pour mettre au point une méthode de travail valable avant d'étendre notre enquête à d'autres régions. La Pologne, la France et le Canada nous semblaient fournir l'occasion de comparaisons Est-Ouest intéressantes et l'enquête paraissait réalisable rapidement. Les questions retenues avaient été choisie en tenant compte des préoccupations des populations de chaque pays. Par exemple, il avait paru nécessaire, en considération des familles canadiennes, d'accorder une place plus importante qu'on ne l'avait prévu aux problèmes d'éducation des enfants liés à l'évolution de la situation de la femme. Après cette confrontation, les trois responsables revinrent à leurs terrains d'étude, pleins d'espoir et de bonnes intentions.

Troisième phase. Pour des raisons d'organisation pratique, l'enquête canadienne dut être retardée. Cependant, les Polonais et les Français se mettaient à l'œuvre avec des moyens financiers obtenus dans leurs pays respectifs. Très rapidement, il apparut que le questionnaire commun devait être sérieusement transformé. Pendant ce temps, en France, le personnel chargé de l'enquête avait changé, par suite de déplacements. Disposant, grâce à l'appui du Conseil supérieur de la recherche scientifique et du 
Conservatoire des arts et métiers, de moyens plus importants que ceux qui avaient été prévus, nous avions décidé d'étendre immédiatement l'enquête à plusieurs milieux différents et d'interroger systématiquement l'homme et la femme dans la même famille ${ }^{1}$. Au cours d'un échange de lettres avec nos collègues polonais, nous décidâmes de garder certaines questions communes et de faire porter les comparaisons principalement sur les milieux ouvriers, comme il avait été prévu au départ. L'enquête française, plus étendue, pouvait préparer le terrain à d'autres recherches ultérieures.

L'enquête polonaise se déroula alors sur les deux terrains que décrivent les articles présentés dans ce numéro de la Revue, sous la direction de A. Kloskowska et J. Piotrowski. On réalisa l'enquête française sur quatre points de la région parisienne, en interrogeant des sujets de trois milieux sociaux différents.

Quatrième phase. Tandis que les dépouillements de ces enquêtes continuaient à s'opérer, il parut possible d'aborder de nouvelles régions d'étude en Afrique blanche et en Afrique noire et de poursuivre la préparation au Canada. Un premier voyage au Maroc (P. Chombart de Lauwe) permit de s'assurer la participation de divers collègues marocains et français qui s'intéressaient à ce sujet et de procéder à une première série d'interviews et de discussions de groupe. Un séjour de plusieurs mois permit ensuite à N. Forget de mettre au point, avec des Marocains, un questionnaire adapté à cette nouvelle culture, de le faire traduire en arabe et de mener toute l'enquête dont l'article dû à N. Forget décrit une partie.

En Afrique noire, N'Sougan Agblemagnon traçait les grandes lignes de l'enquête au Togo tandis qu'un nouveau chercheur, R. Clignet, qui avait déjà mené avec des collègues ivoiriens d'autres recherches suivant une ligne proche de la nôtre, proposait de reprendre les documents réunis et d'en tirer parti pour préparer une nouvelle phase d'étude. Ces travaux rejoignaient ceux qui avaient été entrepris dans d'autres régions d'Afrique sous la direction de D. Paulme. Les efforts devaient naturellement être coordonnés. En même temps, d'autres chercheurs - L. Rosenmayr, Ishwaran - qui n'avaient suivi les projets qu'au début, pouvaient reprendre leurs travaux et les collègues d'autres pays, comme R. Clignet, pouvaient communiquer des expériences intéressantes dans ce domaine.

De cette façon, la ligne des comparaisons Est-Ouest (Pologne-Canada-France) se croisait avec celle des comparaisons Nord-Sud (pays européens et pays africains en voie de développement). La gamme des cultures et des situations économiques était suffisante pour donner une idée des principaux problèmes à résoudre.

Cinquième phase. L'idée d'une présentation des travaux en cours nous est apparue alors avec une force de plus en plus grande. L'enquête française, qui a bénéficié d'une aide plus importante, donnera lieu prochainement à la rédaction d'un ouvrage, dont l'article de M. J. Chombart de Lauwe présente un premier aspect. Les enquêtes polonaises sont intéressantes en elles-mêmes, indépendamment de toutes les comparaisons qu'elles permettent. L'enquête marocaine offre l'exemple d'un troisième niveau de recherche et pose de nombreux problèmes relevant de l'observation sociologique, propres aux pays en voie de développement. Les articles canadien, togolais, ivoirien, etc., ouvrent des perspectives nouvelles et montrent comment un dialogue fructueux peut s'établir en cours d'opération. Enfin l'apport de L. Rosenmayr et de O. Buric, qui ont bien voulu orienter une partie de leurs enquêtes en cours dans le sens de nos

1 Voir l'article de M. J. Chombart de Lauwe, p. 26. 
préoccupations, ouvre des possibilités nouvelles - et capitales - aux développement ultérieurs.

Du point de vue méthodologique, nous avons donc été amenés à organiser des enquêtes coordonnées plutôt qu'une seule enquête internationale et nous pensons que cela est profitable. Les matériaux sont recueillis en fonction de certaines hypothèses communes et en vue d'aboutir à des comparaisons, mais, sur chaque terrain, les opérations se déroulent d'une manière originale, compte tenu du contexte culturel. Chaque élément observé doit être replacé dans une culture particulière dont il constitue une partie et, ensuite seulement, comparé aux autres éléments de même nature observés dans d'autres cultures avec les mêmes précautions.

\section{Deux images dynamiques : la femme au travail et l'égalité des sexes}

En fonction de ces préoccupations méthodologiques et de l'élaboration progressive des instruments de travail, les résultats qui peuvent être présentés dès maintenant sont forcément limités. Mais, en liaison avec l'ensemble des problèmes qui sont soulevés dans tous les pays au sujet de l'évolution du statut de la femme, deux images méritent une attention particulière : celle de la femme au travail et celle de l'égalité des sexes.

\section{a) L'image de la femme au travail}

\section{$\underline{\text { Retour à la table des matières de l'introduction }}$}

L'image de la femme au travail fait déjà ressortir entre les pays des différences instructives. Les ouvriers polonais voient plus facilement que les ouvriers français la femme, mariée ou non, exercer une activité professionnelle. Mais, lorsqu'il s'agit d'une mère avec des enfants en bas âge, l'opposition est quasi unanime, aussi bien en France qu'en Pologne (95\% d'un côté, 96\%, de l'autre). Au Maroc, les oppositions au travail sont plus nombreuses, en raison d'images traditionnelles très vivantes, mais les changements sont extrêmement rapides. Au Canada, l'image de la femme exclusivement mère et épouse paraît plus fixée dans la structure même d'un pays à la fois économiquement très développé et en pleine expansion.

Les raisons qui poussent les femmes à travailler sont, avant tout et partout, d'ordre économique (c'est le cas pour la quasi-totalité des sujets interrogés en France), mais, si, dans les milieux les moins favorisés, il s'agit de survivre, dans les milieux aisés, la 
femme désire gagner de l'argent pour se permettre, et permettre à sa famille, d'avoir plus de moyens pour les loisirs, la culture, les distractions.

Les autres raisons sont bien moins souvent évoquées. Elles révèlent, en général, des oppositions entre les hommes et les femmes interrogés. L'ennui à la maison et le goût pour le métier ne tiennent, dans les divers pays qu'une place assez faible dans les motivations exprimées. Le désir d'indépendance n'apparaît que de façon très limitée au Maroc. Peut-être joue-t-il un rôle plus important en Afrique noire. En France, il est le privilège des femmes des milieux aisés qui, seules, ont une véritable liberté de choix en raison de leur niveau de vie plus élevé. Nous touchons ici au problème essentiel, que nous avons souvent signalé, des rapports entre les contraintes matérielles et la limitation des aspirations. En Pologne, les femmes voudraient souvent travailler, mais relativement peu nombreux sont les maris qui approuvent ce désir. Les femmes qui travaillent veulent, en général, cesser de le faire, tandis que celles qui n'ont pas de travail voudraient souvent en avoir. En France, nous retrouvons des différences du même genre dans les milieux ouvriers, mais les mauvaises conditions de travail, les bas salaires, le «salaire unique » s'ajoutent aux modèles traditionnels pour faire préférer aux femmes de ces milieux la vie de la maison. Au contraire, dans les milieux aisés, les préférences des femmes vont au travail extérieur, quelle que soit leur situation actuelle.

Les inconvénients du travail féminin sont surtout signalés par les hommes. La promiscuité entre les sexes, le danger de mauvaise tenue, sont des arguments qui ont une grande importance au Maroc, et sont loin d'être négligeables en France (avec d'ailleurs des nuances qui varient suivant les milieux). Le refus d'être commandé par des femmes est également plus fréquent au Maroc, mais s'exprime ailleurs. Un peu partout le fait de négliger les enfants et la maison est mis en avant. D'une manière générale, l'ensemble de ces objections donne facilement mauvaise conscience aux femmes, même dans les pays industrialisés.

En résumé, nous pourrions dire que l'ensemble des sujets interrogés auraient du travail féminin, l'image guide suivante : la femme célibataire doit travailler (sauf les oppositions dues à la préparation de la jeune fille au rôle traditionnel d'épouse, telles qu'elles apparaissent encore au Maroc et chez quelques ouvriers polonais, et la crainte de certains milieux aisés français de voir la femme acquérir trop facilement une indépendance économique), la femme mariée sans enfants peut travailler (parfois avec certaines réserves de la part des hommes), la femme mariée avec des enfants en bas âge ne doit pas travailler, sauf cas exceptionnels. Nous verrons plus loin les conséquences de cette vision sur l'organisation du travail et sur la planification.

\section{b) La question de l'égalité de la femme et de l'homme dans la famille et dans la société}

$\underline{\text { Retour à la table des matières de l'introduction }}$

Cette question se pose à propos du travail et à propos d'autres activités. L'influence de la pratique d'un métier sur l'égalité entre les sexes, à la maison, est reconnue dans les pays où le travail féminin est assez développé et elle est parfois redoutée. De même, il existe une tendance fréquente à réclamer des droits égaux dans l'entreprise, 
lorsque la femme travaille. Le travail apparaît volontiers comme un moyen de réparer une injustice et de donner plus de « dignité » à la femme dans la vie sociale.

L'apport d'un salaire par la femme tend à bousculer partout les rôles économiques traditionnels. En Pologne comme en France, la gestion du budget, qui revenait à la femme dans les familles ouvrières (plus souvent pour des raisons de manque d'intérêt de la part de l'homme que du fait de la prédominance systématique de la femme) lui échappe progressivement. Les responsabilités et les tâches de la vie économiques sont de plus en plus partagées également. Au Maroc et en France, dans les milieux à revenus élevés, nous arrivons plus lentement au même résultat, en partant de la situation inverse ' puisque les femmes ne s'occupaient que peu ou pas du tout des questions financières.

Avec l'image de la femme, bonne ménagère, d'abord, puis bonne mère et ayant bon caractère, les Polonais mettent en parallèle une image du père qui doit surtout gagner l'argent de la famille, donner le bon exemple, être attaché à la maison et, en dernier lieu, s'intéresser à l'éducation des enfants. Les images françaises, en milieu ouvrier, ne sont pas tellement différentes, et le Canada offrira sans doute des images plus traditionnelles encore. Au Maroc, intervient l'influence de la doctrine religieuse qui admet difficilement qu'on laisse à la femme des responsabilités manifestes, tout en reconnaissant à la mère un rôle capital dans l'éducation.

La persistance de ces images explique, en partie, l'inquiétude des hommes et, dans une large mesure, des femmes devant le bouleversement de la hiérarchie sociale et le changement des rapports sociaux entre les sexes, Les enquêtes française et marocaine sont particulièrement explicites à ce sujet. La peur d'une perte de prestige de l'homme au sein de la famille semble rare chez les ouvriers polonais. Mais, en fait, les appréhensions des sujets de l'échantillon français sont plus complexes. La femme se défie elle-même de sa propre agressivité, qui se libère à l'occasion du nouveau statut qui lui est donné. C'est l'harmonie du couple qui est mise en cause et la possibilité d'un amour véritable qui semble échapper. Ici apparaissent les conséquences du décalage entre les images et les comportements, sur lesquelles nous reviendrons plus loin.

\section{Problèmes généraux d'évolution et perspectives}

$\underline{\text { Retour à la table des matières de l'introduction }}$

Ces quelques remarques laissent déjà entrevoir des possibilités de comparaison. Mais ce sont surtout les problèmes généraux d'évolution posés par une telle enquête sur les images qui doivent retenir notre attention. La transformation du statut de la femme lors du passage des sociétés traditionnelles aux sociétés industrielles, la perception de cette évolution et les aspirations qu'elle fait naître, l'influence des images dynamiques sur la planification sont autant de sujets de réflexion que nous devons aborder. 


\section{a) Traditions culturelles et universalisme}

$\underline{\text { Retour à la table des matières de l'introduction }}$

Suivant les théories, les auteurs ou les pays, de nombreuses remarques convergentes ont été faites sur les conséquences des échanges culturels. Qu'il s'agisse du passage des « sociétés closes » aux «sociétés ouvertes », de la «communauté » à la « société », des «traditions particulières » à l'universalisme, « des » civilisations à « la » civilisation, il existe une croyance générale à l'apparition de formes nouvelles de culture qui dépassent les frontières des groupes humains fermés. Mais l'étude de la gestation des images nouvelles qui orientent ces transformations présente des difficultés qu'il importe de mettre en relief.

La notion de totalité culturelle reste valable pour l'observation des aspects de la vie sociale liés à la tradition. Elle ne l'est plus pour les autres, à moins qu'on ne considère l'humanité dans son ensemble comme un tout. De là est née une discussion sans objet entre les ethnologues et les sociologues. Sans doute ne pouvons-nous étudier l'image de la mère dans la société marocaine qu'en liaison avec tous les autres traits culturels propres à cette société, qui doit d'ailleurs être étudiée dans le cadre de l'ensemble des sociétés de civilisation musulmane. Mais l'image de la femme au travail qui interfère avec celle de la mère ne peut être mise en relief que comparativement avec des sociétés parvenues à un stade de développement industriel plus élevé. Nous devons donc travailler constamment à plusieurs niveaux de comparaison.

Cela dit, les comportements des hommes et les images qui les orientent sont intimement liés aux milieux sociaux dans lesquels ils vivent quotidiennement. Comme nous l'avons signalé ailleurs, les milieux sociaux nouveaux ne recouvrent plus les anciennes divisions de groupes et de cultures. Ils sont, dans les sociétés en transformation, des réalités mouvantes qu'il est possible d'analyser avec des méthodes d'observation appropriées. Les classes sociales sont souvent plus proches de tels milieux sociaux que de véritables groupes, au sens habituel du mot, mais elles n'en ont pas moins une existence certaine et une influence déterminante dans l'évolution des sociétés industrielles.

Dans ces conditions, les traits culturels propres à un groupe particulier ou à un milieu social donné peuvent revêtir une importance aussi grande que les traits culturels qui caractérisent une société. Les notions de subculture et de culture deviennent de plus en plus relatives. Il existe de moins en moins une image de la femme française ou de la femme américaine, dans la représentation des habitants de la France ou des États-Unis, mais de plus en plus des images différentes suivant les milieux. C'est ce que l'enquête française a voulu commencer à démontrer.

Par contre, il existe, dans l'ensemble du monde actuel, des types de tendances, des images dynamiques opposées, auxquelles les populations se rallieraient plus ou moins volontiers. Pour certains aspects du statut de la femme, la Chine et l'Espagne représenteraient, par exemple, les pôles extrêmes. C'est peut-être sur des pays de ce genre, 
encore plus différents entre eux que ceux où ont eu lieu les premières enquêtes, qu'il faudrait faire porter les observations futures. Mais les difficultés vont alors croissant.

\section{b) Oppositions entre les images dynamiques et les comportements}

$\underline{\text { Retour à la table des matières de l'introduction }}$

En fonction même des influences diverses auxquelles les sujets étudiés se trouvent soumis, nous avions insisté, dès les premières discussions préparatoires à l'enquête, sur le divorce entre les situations vécues et les images de référence.

Ce fait nous avait déjà frappé dans des enquêtes précédentes qui portaient, en particulier, sur les attitudes des parents à l'égard des enfants. Le problème a été bien mis en relief dans la présente recherche internationale par plusieurs chercheurs, notamment par A. Kloskowska qui s'y attarde longuement, et avec laquelle nous sommes à peu près entièrement d'accord. Il resterait à mettre au point une question de vocabulaire. La distinction entre les «modèles» conscients et normatifs et les patterns correspondant à des « comportements socio-culturels » authentiques est bien conforme à ce que nous avons nous-même observé.

Nous distinguerons aussi les modèles construits volontairement et consciemment par la propagande, des modèles qui sont élaborés spontané ment par les groupes ou qui naissent dans les milieux sociaux. Cependant, nous avons signalé plus haut que les influences de plusieurs modèles différents, liés à la tradition et aux courants nouveaux, pouvaient se rencontrer dans une « image » à laquelle se réfèrent les sujets dans leurs comportements - ces images dépendant aussi de la manière dont les sujets « perçoivent » les situations et les structures sociales. En fonction de ces influences diverses, elles contiennent en elles-mêmes des contradictions et doivent être distinguées des modèles qui les font naître et des «formes » de comportements (patterns of behaviour). Or ce sont ces images complexes que nous atteignons dans l'observation et auxquelles se réfèrent directement les sujets; c'est pourquoi nous avons déjà, dans des enquêtes précédentes, insisté sur leur importance et leur force active.

Le point capital est alors la rupture, que confirment la plupart des rapports présentés, entre les images de référence, dans lesquelles les éléments traditionnels sont souvent dominants, et les comportements de fait liés à des situations vécues. Ainsi la femme marocaine qui travaille peut-être quelquefois «honteuse » d'avoir à le faire parce que son image de référence reste la mère traditionnelle, cantonnée à la maison. Au contraire, un représentant des classes moyennes, en France, peut se référer à une image nouvelle de la femme au travail, qu'il accepte sincèrement, tout en désirant que sa propre femme reste au foyer dans la situation présente, en raison des circonstances. Si ces décalages ne sont pas bien saisis dès le départ des enquêtes, ils peuvent entraîner des erreurs considérables dans l'interprétation des résultats. 
La contradiction du même genre signalée par J. Piotrowski entre les opinions recueillies dans les enquêtes et la réalité existant dans les familles ne doit pas lui faire émettre un doute sur la validité de la recherche. Cette contradiction a, au contraire, un sens précis et confirme certaines hypothèses de travail. Il en est de même des variations d'opinion en liaison avec les changements de situation qui soulignent les rapports intimes entre le milieu social et les comportements. L'enquête n'est pas une enquête d'opinion; elle étudie les images, leur perception et leur influence sur les comportements. Mais cela demande d'autres développements sur la perception.

\section{c) La perception des situations et la perception de l'évolution}

Nous arrivons au point central de l'étude, telle qu'elle se déroule actuellement dans l'enquête française en particulier (M. J. Chombart de Lauwe). Nous avons insisté sur l'influence de la perception dans la genèse des images guides, mais la perception de l'évolution joue plus encore que la perception des situations actuelles. La prise de conscience des changements déjà opérés, à laquelle aboutissent les sujets, entraîne la vision d'autres changements possibles. Des aspirations nouvelles apparaissent, en même temps que des inquiétudes et des craintes. Nous atteignons l'aspect fondamental du processus. C'est en fonction de cette perception de l'évolution qu'une femme peut espérer se libérer d'une situation oppressante, mais, en même temps, elle peut craindre les conséquences dont nous avons parlé pour la vie de son ménage. Il ne faudra donc pas s'étonner que nous ayons donné jusqu'ici et que nous soyons décidés à donner plus encore dans l'avenir une place prioritaire à cette partie de la recherche.

\section{La nouvelle image de la femme dans la société à venir}

$\underline{\text { Retour à la table des matières de l'introduction }}$

Dans l'ensemble de l'enquête internationale, comme dans la littérature, la presse ou le cinéma, un fait se dégage clairement : la naissance d'une nouvelle image de la femme dans la société. Cette image déclenche des passions, car elle implique à la fois une modification des rapports entre les sexes et une transformation de l'ensemble des structures sociales. Comment, dans ces conditions, la recherche entreprise doit-elle être poursuivie? 


\section{a) La nouvelle image de la femme et l'avenir du couple humain}

\section{$\underline{\text { Retour à la table des matières de l'introduction }}$}

La femme a pris sa place dans la vie de travail, non plus comme la collaboratrice de l'homme dans une petite exploitation agricole, mais comme un travailleur normal dans l'entreprise industrielle. Ce principe semble admis progressivement, en liaison avec le développement technique. Pour la femme célibataire, il n'est plus combattu que par une minorité. Mais une série de conditions sont posées dans le travail même, en fonction des qualités ou des défauts attribués à la femme, de l'organisation du travail, surtout des soins à donner aux enfants, des rapports entre les sexes et de la conception de l'égalité.

Les enquêtes ont fait ressortir, nous l'avons dit, une vision schématique du travail de la femme suivant trois états : la femme célibataire doit travailler, la femme mariée sans enfants ou avec de grands enfants peut travailler, la femme mariée avec des enfants en bas âge ne doit pas travailler. Cette vision paraît refléter une certaine sagesse, mais elle est difficilement conciliable, dans la pratique, avec les principes d'égalité exprimés par ailleurs, car les restrictions tendent fatalement à limiter les possibilités de progression dans un métier.

Cependant, la notion d'égalité totale des sexes gagne manifestement du terrain. Les hommes et les femmes en sentent la vérité, mais ils en ont peur chacun à leur manière. Le droit de vote est admis plus facilement que le droit au travail ou la possibilité de commander, parce qu'il bouleverse moins directement les rapports traditionnels entre les sexes. La révolution qui s'opère est beaucoup plus profonde, beaucoup plus intime, aussi ne faut-il pas s'étonner, comme nous l'avons dit au début, qu'elle suscite tant de passions. Les « contre-mythes » féministes opposés aux mythes traditionnels de la virilité créent un climat dans lequel les arguments rationnels perdent de leur efficacité. C'est finalement dans la notion du couple qu'il faut chercher la clé du problème.

A quoi servirait à l'homme de préserver sa « supériorité », à quoi servirait à la femme de gagner sa « liberté » s'ils se sentaient par là même étrangers ou ennemis ? C'est pourquoi l'accord sur une image de référence acceptée par les deux sexes est si important pour l'avenir. Bien des femmes ont exprimé la crainte que leur inspiraient leur propre agressivité et leur désir de revanche. Une vieille rivalité entre les sexes ressort du fond des temps. Comment la dépasser aujourd'hui au lieu de la faire renaître plus aiguë que jamais ? À l'intérieur de la famille, le partage ,des responsabilités progresse, le dialogue du couple change et s'enrichit, l'égalité s'affirme progressivement, mais, répétons-le, les différences demeurent et la vie du couple n'est possible que grâce à elles.

Ici pourrait s'ouvrir de nouveau le débat sur la nature et la culture. Nous conduirait-il très loin ? En termes d'évolution, le problème se pose autrement. L'évolution biologique et l'évolution sociale ne sont pas opposées. D'un côté comme de 
l'autre, on peut concevoir une progression de l'égalité dans la différenciation; on ne peut guère comprendre une progressive similitude qui aboutirait, en fin de compte, à une définitive solitude.

\section{b) La nécessaire transformation des structures sociales}

$\underline{\text { Retour à la table des matières de l'introduction }}$

L'inquiétude ressentie par les hommes et les femmes interrogés ne peut prendre fin que si les conditions d'une véritable libération de la femme et les véritables possibilités d'une communion réelle dans le couple sont réalisées. La transformation de l'ensemble des structures sociales, dont nous avons souligné la nécessité, doit être effectuée en relation avec la nouvelle image de la femme.

L'organisation du travail doit être repensée. L'aménagement des horaires, des congés, des échelles de salaires, des rapports sociaux dans les ateliers, de la répartition des tâches, de l'orientation professionnelle, de la promotion sociale, etc., doit tenir compte des besoins et des aspirations liés à la nouvelle image de la femme. De même, la vie sociale dans les quartiers résidentiels doit être réorganisée pour permettre à la femme d'être libérée de nombreuses tâches qui l'écrasent actuellement. Les équipements sociaux, sanitaires, culturels et surtout éducatifs doivent être développés. La conception des crèches, des garderies, des maternelles, des écoles, des maisons de jeunes dépendra du rôle que l'on attribue à la femme.

La conception de l'enseignement et de l'éducation est liée intimement, elle aussi, à l'image de la femme. Les études entreprises à ce sujet en France par V. IsambertJamati sur l'éducation des filles en relation avec l'évolution des rôles de l'homme et de la femme apporteront sans doute des éléments nouveaux de réflexion, en liaison avec les enquêtes dont nous parlons aujourd'hui, De toute façon, la répartition des tâches entre l'école et la famille dépend, dans une large mesure, du rôle de la femme dans l'éducation.

L'évolution des structures et des fonctions de la famille, telle que nous l'étudions dans d'autres recherches, est, elle aussi, orientée avant tout par la conception du rôle de la femme dans la société. La vie du couple et l'organisation de la phratrie dépendent de la situation de la femme. Le rôle du père lui-même change complètement si la femme prend en charge de nouvelles responsabilités. Or nous connaissons l'importance de l'image du rôle du père dans l'évolution des rapports sociaux. L'autorité dans la famille et l'autorité dans la société ont trop souvent été rapprochées pour qu'il soit utile d'y revenir ici.

Toutes ces transformations impliquent une modification des lois. Nous avons fait allusion, à propos des pays étudiés, aux changements intervenus dans les codes polonais et marocain, par exemple. Mais bien d'autres modifications interviendront dans tous les pays, avant que des solutions durables soient trouvées. Les curieuses discussions qui ont eu lieu il n'y a pas si longtemps dans divers milieux parlemen- 
taires montrent que les images traditionnelles sont plus vivaces qu'on ne l'aurait cru dans des pays depuis longtemps industrialisés.

\section{c) Développements ultérieurs des recherches}

$\underline{\text { Retour à la table des matières de l'introduction }}$

L'importance des recherches sur la perception de l'évolution du statut de la femme, sur les modèles, les formes de comportements, les images guides qui s'y rapportent, apparaîtra mieux, nous l'espérons, après ces quelques remarques, si brèves soient-elles. Il reste cependant à rappeler les limites de l'étude qui tiennent, en quelque sorte, au fait que les recherches sont liées à l'évolution de toutes les structures sociales.

Ce qui compte pour nous, c'est la conscience de l'évolution de la société dans l'esprit des hommes et des femmes des différents pays. Les enquêtes présentées mettent en relief le rôle de la perception et de la modification des images de référence dans l'apparition des aspirations et dans la transformation sociale. Mais il est alors nécessaire de relier l'image nouvelle de la femme dans la société à celle de la famille de demain, à celle de l'enfant idéal, à celle de la ville future, à celle des classes sociales, de l'État, de la religion, etc. Les enquêtes internationales devront se développer, compte tenu de ces liaisons nécessaires. Les essais que nous soumettons actuellement au public ne prétendent apporter qu'un élément de réflexion dans une entreprise beaucoup plus vaste, dont il faudra définir progressivement le cadre et l'orientation. 


\section{I \\ Image de la situation de la femme dans la société urbaine française}

Étude auprès d'ouvriers et de représentants des petites classes moyennes Une enquête collective du Groupe d'ethnologie sociale

\section{J. Chombart de Lauwe}

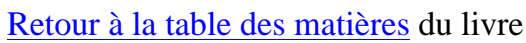

Table des matières de l'article sur la femme en France

Introduction

1. La méthode

a) Les techniques d'enquête

b) Le choix de l'échantillon

c) Les caractéristiques des échantillons ouvriers et intermédiaires

2. Les attitudes à l'égard de la condition actuelle
a) Le travail professionnel de la femme

3. Les préoccupations économiques et les aspirations réelles des femmes

a) La société. La femme et le couple. Le principe de l'égalité des sexes

4. La perception de l'évolution du statut de la femme en fonction des changements des structures sociales et des conditions de vie
a) Les changements des droits
b) L'ouverture de nouveaux débouchés pour les femmes
c) Les changements dans la vie familiale 
5. La perception des changements de comportement des deux sexes a l'égard l'un de l'autre

a) La perception des changements en fonction du sexe, de l'âge et du milieu social, d'origine et actuel

6. La nature de cette évolution

a) Les conséquences. La femme est-elle plus heureuse aujourd'hui ?

Conclusion

L'enquête française, présentée en premier, est celle qui a disposé des moyens les plus importants, grâce à l'apport du Conseil supérieur de la recherche scientifique et du CNRS. Elle a servi d'expérience pilote parallèlement à l'enquête polonaise. L'ensemble des résultats, qui débordent largement le cadre de cet article, figurera dans un ouvrage à paraître en 1962, qui offrira d'autres éléments de réflexion en vue des recherches futures.

\section{Introduction}

$\underline{\text { Retour à la table des matières sur la femme en France }}$

La manière dont les hommes perçoivent et se représentent la société et l'évolution des structures sociales a été l'un des thèmes centraux de recherche du Groupe d'ethnologie sociale. L'étude dont nous exposons ici les résultats s'inscrit dans cette ligne. En effet, les modifications que l'on constate dans les attitudes à l'égard du statut de la femme sont le signe de transformations des structures de la famille et, plus largement, de la société.

Depuis quelques années se manifeste un intérêt de plus en plus vif pour les problèmes touchant à l'émancipation de la femme. Certains articles qui traitent de la question sont malheureusement fort peu objectifs et traduisent les préoccupations des auteurs de façon souvent très passionnelle.

D'autre part, diverses études portant sur les conditions de vie des femmes (travail, horaires, etc.), sur leur participation effective à la vie sociale ou politique ou leur rôle dans la famille, ont été effectuées et publiées. Il s'agit ici, pour nous, de mettre en relief non les situations elles-mêmes, mais les attitudes à l'égard de ces situations.

C'est pourquoi il nous a semblé utile d'étudier de façon systématique la manière dont les hommes et les femmes de différents milieux se représentent la place de la femme dans la société. 


\section{La méthode}

$\underline{\text { Retour à la table des matières sur la femme en France }}$

L'étude a donc pour but de rechercher comment évoluent des attitudes et comment se modifient certaines perceptions en fonction de données précises du milieu. Pour cela, on pourrait analyser, dans un milieu relativement constant, les modifications qui se produisent au cours du temps, sous l'influence de courants de pensée nouveaux. On pourrait aussi étudier, à un moment donné, les différences liées à des variations du milieu. C'est surtout cette dernière méthode que nous avons retenue ici. Cependant, au cours d'enquêtes antérieures faites pair le Groupe d'ethnologie sociale sur des milieux identiques, nous avions abordé les mêmes thèmes. Certaines comparaisons dans le temps ne sont donc pas exclues, mais elles sont limitées.

Nous nous appuyons sur une enquête qui permet des études en profondeur sur des ménages et des comparaisons entre divers milieux. L'échantillon est un échantillon stratifié au hasard. Chaque questionnaire constitue à lui seul une petite monographie. Il évite l'inconvénient des études de cas, puisque chaque sujet interrogé est situé à l'intérieur d'un échantillon choisi suivant des critères précis.

\section{a) Les techniques d'enquête}

Chaque enquête portant sur un ménage est composée de deux interviews simultanées du mari et de la femme. Pour éviter que les deux sujets interrogés ne se communiquent les questions et les réponses, un enquêteur interroge l'homme, tandis que sa collègue pose les questions équivalentes à la femme. Cette exigence a rendu bien souvent la tâche des enquêteurs très difficile. Une telle formule serait inapplicable à une enquête extensive, mais elle permet des comparaisons entre les attitudes spontanées des hommes et des femmes, qu'on ne pourrait établir autrement avec autant de certitude,

Après avoir tiré au sort les ménages, les enquêteurs leur adressent une lettre en sollicitant un rendez-vous à leur domicile et en leur expliquant le but et l'intérêt de l'enquête. Les motifs de refus sont enregistrés et font l'objet d'une étude systématique.

Le questionnaire comprend des questions tout à fait ouvertes et des questions semi-fermées, comportant une série de propositions (établie d'après des analyses de contenu de questions identiques posées de façon ouverte dans des enquêtes antérieures). Il est possible de répondre par une nouvelle proposition.

L'enquête dure environ une heure et demie. Une fois qu'elle est terminée, les enquêteurs joignent aux réponses, transcrites aussi exactement que possible, des 
commentaires permettant une meilleure compréhension de l'ensemble. Ils y ajoutent un résumé, décrivant la famille et l'ambiance de l'interview. Si des sujets dépassant le cadre précis de l'enquête sont abordés, l'enquêteur rédige des fiches d'observation par thème, lesquelles seront ensuite regroupées.

À côté de ces enquêtes portant sur des couples, un échantillon formé d'étudiants (filles et garçons) a également été interviewé. Enfin, nous avons procédé à des enquêtes collectives, à l'aide d'un questionnaire restreint, avec des questions fermées et en profitant de réunions du syndicat des familles et de groupes de l'école des parents. Des feuilles de réponses avaient été distribuées aux participants, et les questions, auxquelles ils devaient répondre par écrit, étaient lues oralement.

\section{b) Le choix de l'échantillon}

Retour à la table des matières sur la femme en France

En fonction des études antérieures du Groupe d'ethnologie sociale, divers critères ont présidé au choix de cet échantillon stratifié :

Le niveau socio-économique. Nous avons réalisé un échantillon ouvrier, un échantillon intermédiaire et un échantillon à revenu élevé. Chacun des échantillons comprend 60 ménages, ce qui représente 120 questionnaires. L'échantillon étudiant comprend 100 sujets, ce qui donne un total de 460

Le quartier. On réalise chaque échantillon de ménages en en choisissant une moitié dans un quartier de type ancien, à l'intérieur de Paris, et l'autre dans un quartier de type nouveau, en banlieue.

La présence d'enfants. Tous les ménages choisis doivent avoir des enfants. Le tirage au sort des familles a donc été effectué d'après des listes de recensement en banlieue et d'après des listes administratives à Paris. Les cas possibles sont déterminés dans ces listes grâce aux critères indiqués, puis un tirage est effectué entre ces cas. Lorsqu'il y a refus, le ménage est systématiquement remplacé par un autre qui présente les mêmes caractéristiques.

\section{c) Les caractéristiques des échantillons ouvriers et intermédiaires}

$\underline{\text { Retour à la table des matières sur la femme en France }}$

Les échantillons ainsi réalisés ont des caractéristiques qui motivent parfois les attitudes à l'égard de la condition de la femme, ou, tout au moins, leur sont liées.

La résidence. La moitié des membres de l'échantillon «ouvrier » et la moitié de ceux de l'échantillon « intermédiaire » habitent dans deux petits quartiers du treizième 
arrondissement, les autres dans une cité de type HLM à Villejuif. Le nouveau style de vie qu'imposent les cités nouvelles ou le simple dépaysement permet aux sujets interrogés d'envisager plus facilement des formules nouvelles et les rend souvent plus favorables à l'émancipation de la femme.

Profession des hommes et branches professionnelles. Les trois quarts des ouvriers sont au moins O.S.2, ou ont des qualifications diverses. Plus des deux tiers des ouvriers $(68,3 \%)$ appartiennent aux grandes branches professionnelles -métallurgie, $35 \%$, transports, $20 \%$. [RATP, SNCF, Air France (4cas)], imprimerie et papeterie. Or ces branches professionnelles comptent parmi les plus dynamiques et les plus représentatives du monde ouvrier ; 8,3\%. des membres de l'échantillon ouvrier travaillent dans le bâtiment, 5\%, dans des entreprises de matière plastique, $5 \%$ également sont au service d'artisans et de petits commerçants. Nous avons regroupé en un poste « divers » 5 ouvriers appartenant à des branches professionnelles qui ne correspondent pas à des secteurs ouvriers classiques, par exemple un électricien attaché à une banque, ou un autre à l'hôtellerie, etc. Il existe peu de différence entre les échantillons de Villejuif et ceux du treizième arrondissement en ce qui concerne ces cas divers. La branche des transports est davantage représentée à Villejuif, alors que la papeterie et l'imprimerie se regroupent surtout dans le treizième. La proportion des représentants des autres branches professionnelles est la même dans les deux quartiers.

En revanche, l'appartenance au quartier sépare les membres de l'échantillon intermédiaire en deux groupes, dont les caractéristiques sont différentes du point de vue professionnel. Le treizième est marqué par la présence d'un groupe important d'artisans $(23,3 \%$ et de quelques commerçants. Parmi les autres sujets interrogés, 26,7\% travaillent dans l'industrie (métallurgie, aviation, automobile, béton armé, machines mécanographiques, papeterie). Les branches professionnelles sont nombreuses et diverses dans ce quartier. Nous citerons : l'hôtellerie, le bâtiment, l'enseignement, la préfecture de police, l'armée, les assurances, l'importation-exportation, les soins hospitaliers, etc.

La cité de Villejuif se présente avec beaucoup plus d'unité. Des sujets interrogés, 43,3\%. appartiennent à l'industrie, 33,3\% aux transports. Ce sont soit des techniciens ou des ingénieurs, soit des « administratifs ». Les autres sont commerçants ou agents commerciaux, assistants d'expert-comptable, attachés de direction dans une banque, journalistes, techniciens du bâtiment dans une entreprise de travaux publics (un cas pour chaque profession).

L'échantillon intermédiaire reflète fidèlement l'aspect de deux types de quartier : l'ancien, avec la présence de l'artisanat et de métiers divers, le nouveau, avec une plus grande unité sur le plan professionnel et sur le plan du type de famille. Ce sont aussi les branches professionnelles les plus dynamiques qui relogent le plus leur personnel, du fait de leur esprit d'initiative et de leur économie générale. Enfin, peu de locaux à usage commercial sont prévus dans les cités nouvelles, d'où la difficulté pour les artisans de s'y insérer. Dans notre enquête, plus on s'élève dans l'échelle des catégories socio-professionnelles, plus le changement de type de quartier semble entraîner de différences entre les groupes qui y habitent. Les quartiers anciens ont tendance à regrouper des habitants exerçant des métiers de type plus classique, les quartiers nouveaux, ceux qui appartiennent à de nouvelles industries. Au niveau des intermédiaires, les techniciens de l'électronique et de la construction de radars habitent 
Villejuif, les artisans, le treizième arrondissement. Mais c'est surtout au niveau des hauts revenus que nous avons constaté des coupures marquées.

Pourquoi une telle opposition ne se rencontre-t-elle pas aussi dans le milieu ouvrier ? Probablement parce que les ouvriers, étant donné le peu de moyens économiques dont ils disposent, n'ont pas la possibilité de choisir librement le lieu de leur domicile, comme le font plus souvent ceux qui appartiennent à des classes plus favorisées de la population. Il serait alors normal de trouver plus de divergences de comportement et d'attitudes entre les intermédiaires des deux quartiers qu'entre les ouvriers, si des choix relativement libres correspondent à des aspirations différentes. L'hypothèse peut en être posée au début de cette recherche.

Travail et profession des femmes. Les travaux exercés par les femmes de l'échantillon ne présentent pas la même diversité que les travaux masculins. L'appartenance à l'un ou l'autre quartier ne modifie significativement ni le pourcentage des mères de famille exerçant un métier, ni le type de métier exercé. Dans l'échantillon ouvrier, $43,3 \%$ des mères travaillent. Ce pourcentage est fort, car, dans le département de la Seine, le pourcentage des mères de famille qui travaillent n'est que de $22 \%$. Mais le tiers des femmes actives de l'échantillon ouvrier ne travaillent qu'à temps partiel. Les métiers le plus souvent exercés sont ceux de femme de ménage, de petite employée de bureau, d'ouvrière, de gardienne d'enfants - activités classiques dans une population formée de ménages où l'homme est ouvrier.

Pour les intermédiaires, la proportion de femmes exerçant un métier est un peu plus faible (35\%), mais la différence n'est pas significative. Les professions le plus fréquemment mentionnées sont celles de secrétaire ou de comptable dans un bureau, des professions commerciales ou artisanales (souvent en collaboration avec le mari), la profession enseignante, enfin un cas d'assistante sociale.

Nombre et âge des enfants. Ces femmes, du fait de la sélection opérée, ont toutes des enfants, mais, dans l'échantillon ouvrier, elles continuent plus souvent à travailler que dans l'échantillon « intermédiaire », lorsque le nombre d'enfants s'accroît $(19,3 \%$, des femmes actives de l'échantillon ouvrier ont un enfant, $50 \% 2$ enfants et 30,7\% 3 ou 4 enfants; des femmes actives de l'échantillon intermédiaire, $58 \%$ ont un enfant, $25 \% 2$ enfants et $20 \%, 3$ ou 4 enfants.)

En revanche, les femmes, dans l'échantillon ouvrier, ont des enfants un peu plus âgés que dans l'échantillon intermédiaire, ce qui leur laisse plus de facilités pour l'exercice d'un métier. Le pourcentage de femmes exerçant une activité professionnelle est, en effet, ici, directement lié à l'âge des enfants.

Si l'on considère, dans l'ensemble des deux échantillons, la population formée par les enfants des familles interviewées, les plus jeunes (moins de 6 ans) ont des mères qui travaillent dans 22,3\% des cas. Pour les moyens (6 à 14 ans), ce pourcentage s'accroît : (31,8\% et, pour les plus grands (plus de 14 ans), il atteint 53\%. Villejuif et le treizième arrondissement ne se différencient pas sur ces points.

D'une façon générale, les ouvriers ont, dans cette enquête, des enfants un peu plus âgés que les intermédiaires (59\% de plus de 14 ans contre $41 \%$ ). Ils ont aussi des familles plus nombreuses: $16,7 \%$ des ouvriers ont un enfant contre $40 \%$, des intermédiaires, alors que $26,7 \%$ des ouvriers ont 4 enfants et plus contre $13,3 \%$ des intermédiaires. 
Le quartier aussi différencie les familles sur ce point : à Villejuif, les familles de 4 et 5 enfants composent près du tiers des échantillons (30\%) alors que, dans le treizième arrondissement, elles n'en forment que le dixième. Ce que nous indiquons pour l'ensemble des deux échantillons est valable pour l'un et l'autre pris isolément.

Que les familles nombreuses soient davantage regroupées dans la cité de Villejuif peut s'expliquer du fait du relogement, mais aussi du fait de l'âge des familles : les deux phénomènes sont liés. $77 \%$ des hommes à Villejuif et $52 \%$ dans le quartier du treizième ont moins de 40 ans. Les familles du treizième n'ont pas moins d'enfants parce qu'elles sont au début de la période de fécondité, mais, au contraire, parce qu'elles appartiennent, en partie, à une génération plus âgée, qui a été peu féconde. D'ici quelques années, la différence entre les deux quartiers risque d'être encore plus sensible. De ce point de vue, les caractères des deux quartiers sont beaucoup moins marqués au niveau ouvrier qu'au niveau intermédiaire. Nous avions fait la même remarque en observant la répartition des branches professionnelles. Le regroupement des branches les plus dynamiques et les plus jeunes, à Villejuif, correspond aussi au groupe le plus jeune et le plus fécond.

Âge respectif des hommes et des femmes. Les âges des femmes correspondent à ceux des hommes et les variations indiquées pour ces derniers se retrouvent pour l'autre sexe. Nous n'insisterons donc pas sur ce point. Dans l'échantillon ouvrier et dans l'échantillon intermédiaire, les couples sont du même âge, ou l'homme est un peu plus âgé, ce qui est classique. (Voir tableau I)

Tableau I.

\begin{tabular}{lcccc}
\hline & \multicolumn{4}{c}{ Comparaison de l'âge du couple } \\
\cline { 2 - 5 } & $\begin{array}{c}\text { Égal } \\
\text { (ou 1 an de } \\
+ \text { ou de -) }\end{array}$ & $\begin{array}{c}\text { Homme } \\
\text { plus âgé } \\
\text { de 2 à } 9 \text { ans }\end{array}$ & $\begin{array}{c}\text { Homme } \\
\text { plus âgé } \\
\text { de } 10 \text { à } 18 \text { ans }\end{array}$ & $\begin{array}{c}\text { Homme } \\
\text { plus jeune } \\
\text { de } 2 \text { à } 9 \text { ans }\end{array}$ \\
$\begin{array}{l}\text { Échantillon ouvrier } \\
\text { Échantillon intermédiaire }\end{array}$ & $\begin{array}{c}21,7 \\
31,7\end{array}$ & 51,7 & 6,6 & 20 \\
\hline
\end{tabular}

Les différences entre les deux échantillons ne sont pas significatives.

Les conditions de vie: le logement. Du point de vue des conditions de vie, l'enquête a abordé deux problèmes : les conditions économiques, qui seront traitées dans la deuxième partie de cet article, et l'habitat. Les familles relogées à Villejuif ont des logements mieux équipés et moins surpeuplés que dans le treizième. Si les ouvriers se trouvent plus souvent dans des logEments surpeuplés, leur situation est, dans l'ensemble, plus sombre dans le treizième. La moyenne de l'indice (nombre de personnes par pièce) se situe à 2,25 pour les ouvriers du treizième, à 1,47 pour ceux de Villejuif, et, pour les intermédiaires, à 1,42 et 1,33 dans les mêmes quartiers. Les pourcentages de logement en état de surpeuplement critique sont présentés dans le tableau 2. 
Tableau 2.

Logements en état de surpeuplement critique (treizième et Villejuif).

\begin{tabular}{lcccc}
\hline & \multicolumn{2}{c}{ Ouvriers } & \multicolumn{2}{c}{ Intermédiaires } \\
& Treizième & Villejuif & Treizième & Villejuif \\
Surpeuplement temporaire admis & 20 & 23,3 & 26,7 & 23,3 \\
Surpeuplement critique & 63,3 & 13,3 & 26,7 & 10 \\
\hline
\end{tabular}

Donc, plus de $80 \%$ des ouvriers et la moitié des intermédiaires du treizième habitent des logements surpeuplés, tandis que le tiers seulement des uns et des autres vivent dans de telles conditions à Villejuif Cette proportion reste, toutefois, encore très forte si l'on considère qu'à Villejuif il s'agit de familles relogées. Les divergences entre les deux quartiers sont marquées sur ce point et, une fois de plus, elles reflètent typiquement les caractères des quartiers nouveaux et anciens. Nous décrirons d'une façon plus précise, dans d'autres publications, les conditions de logement de ces familles. Nous indiquons seulement ici quelques particularités des échantillons, afin de mieux comprendre les raisons qui pourront les amener, au cours de l'enquête, à adopter telle ou telle position, à avoir telles réactions ou à s'opposer les uns aux autres.

Les attitudes analysées ici seront, d'une part, les attitudes à l'égard du statut de la femme dans la société contemporaine, d'autre part, les attitudes relatives à l'évolution du statut de la femme. Nous observerons en même temps la façon dont cette évolution est décrite, et les jugements de valeur qui sont émis sur les différents changements perçus.

\section{Les attitudes à l'égard de la condition actuelle}

Retour à la table des matières sur la femme en France

Les attitudes à l'égard de la condition de la femme dans notre société peuvent être exprimées en fonction de principes ou de situations vécues. Il en résulte parfois des contradictions intéressantes entre les différentes réponses d'un même sujet interrogé. Certains n'arrivent pas à se détacher de leur propre situation. Plus le niveau d'instruction des sujets interrogés est élevé, et plus vaste est leur niveau d'information, plus ils s'abstraient facilement de leurs conditions personnelles et plus aussi ils nuancent leurs positions. 
Trois points de vue principaux seront abordés dans cette partie : l'attitude à l'égard du travail professionnel, la gestion du budget et les préoccupations économiques, le problème de l'égalité de la femme et de l'homme.

\section{a) Le travail professionnel de la femme}

\section{Retour à la table des matières sur la femme en France}

Au cours de recherches antérieures, les prises de position exprimées pour ou contre le travail professionnel de la femme sont apparues comme reflétant des aspirations à des structures sociales différentes. Les modifications des conditions du travail féminin, le fait que la femme mariée puisse exercer un métier sont à la fois le signe d'une transformation du statut social de la femme et le signe d'une évolution des rôles au sein de la famille. Le travail est considéré parfois comme une association de libération pour la femme et, parfois, comme une forme d'asservissement. Il nous a donc semblé indispensable d'observer les attitudes à l'égard du travail professionnel, non seulement auprès des militants et chez les intermédiaires, mais aussi dans le milieu ouvrier au sens large.

Question de principe. Pour la question de principe, posée «en général » ${ }^{1}$, sans spécifier la situation de famille, les divergences d'opinion entre les hommes et les femmes des deux milieux (ouvriers et intermédiaires) sont très marquées : $21,7 \%$ seulement des hommes, quel que soit le milieu, approuvent l'exercice d'un métier pour la femme, tandis que $65 \%$ des femmes d'intermédiaires et $52,3 \%$ de celles de l'échantillon ouvrier y sont favorables.

Évidemment, le travail n'est pas contesté à la femme seule, célibataire ou veuve sans enfants. Mais l'engagement professionnel de la mère qui a des enfants en bas âge est refusé par la grosse majorité des hommes ou femmes interrogés, la proportion étant la plus forte chez les ouvriers ${ }^{2}$. Lorsque les enfants atteignent l'âge scolaire, l'opposition est encore assez forte, surtout chez les hommes de milieu ouvrier. ${ }^{3}$

La différence de position de principe entre les deux sexes s'exprime surtout à l'occasion de l'exercice d'une profession pour la femme mariée sans enfant. La quasitotalité des femmes l'acceptent ( $81,7 \%$ et $90 \%$ selon le milieu), tandis que leurs maris y sont nettement moins favorables (un peu plus de la moitié des ouvriers, et les deux tiers des intermédiaires sont « pour »).

S'il existe une opposition de principe assez forte au travail féminin chez les hommes, elle est accompagnée d'un désir de voir au moins la femme, quand elle doit travailler, rester dans un milieu de travail qui lui soit propre. La différenciation des rôles entre les deux sexes semble souvent désirée sur le plan professionnel. Les hom-

«Êtes-vous pour ou contre le travail professionnel des femmes?»

5\% seulement des hommes et des femmes acceptent le travail de la femme mariée avec des enfants en bas âge, tandis que, chez les intermédiaires, 13 des hommes et $10 \%$ des femmes l'approuvent.

$3 \quad 16,7 \%$ des hommes et $25 \%$ des femmes seulement approuvent le travail de la femme mariée avec des enfants d'âge scolaire, tandis que, chez les intermédiaires, $20 \%$ des hommes et $30 \%$ des femmes y sont favorables. 
mes de l'échantillon ouvrier souhaiteraient que leurs femmes travaillent à domicile : ils sont mal informés des inconvénients de ce type de travail, ou bien ils tiennent malgré tout à la présence de la mère au foyer. D'une façon générale, les hommes de l'échantillon ouvrier apparaissent comme les plus hostiles au travail professionnel de la femme et à tout ce qui l'éloigne du foyer. À l'autre extrême, les femmes de l'échantillon intermédiaire sont favorables à tout ce qui peut les rendre plus indépendantes et favoriser leur émancipation. Les ouvriers des deux sexes, se référant à des expériences professionnelles pénibles, s'opposent au travail d'usine pour la femme, ou au moins le limitent à certains postes. Les intermédiaires manifestent moins d'hostilité à ce travail, par principe, pour défendre l'égalité des sexes. Parfois c'est le milieu socioprofessionnel qui influence le plus les attitudes, parfois c'est le sexe.

Reproches adressés aux femmes qui travaillent. Dans le travail professionnel, certains reproches sont adressés aux femmes et certaines qualités leur sont reconnues. Ces jugements correspondent, en partie, à des stéréotypes qu'il est intéressant de souligner, car ils tracent un «modèle » de la femme au travail dans notre société. Quelques-uns de ces jugements traduisent des comportements très fréquents des femmes, qui peuvent s'expliquer en fonction des situations dans lesquelles elles vivent. Les reproches le plus souvent énoncés sont les suivants: les femmes sont trop fréquemment absentes, elles sont une cause de disputes, elles prennent la place des hommes, elles n'ont pas le sens de la mécanique, elles sont incapables de travailler en équipe, elles ont un caractère inégal.

L'absentéisme féminin est un fait connu et étudié ; il est dû à la fois aux difficultés que rencontre la femme dans son foyer (obligation de garder un enfant malade que la crèche refuse, etc.) et au fait que la femme n'a souvent pas un sens suffisant des responsabilités dans son métier. Elle s'absente d'ailleurs de moins en moins à mesure qu'elle a un travail plus « qualifié », donc plus intéressant et mieux rémunéré ${ }^{1}$.

De nombreuses remarques d'ordre qualitatif faites par les sujets interrogés montrent que, si les stéréotypes jouent un rôle dans ces jugements, les femmes n'ont pas non plus toujours un comportement adulte dans leur milieu de travail, du fait d'une subordination à laquelle elles ont été très longtemps habituées. Elles sont d'ailleurs refusées comme supérieures ou camarades par la majorité des hommes, et par une proportion importante de femmes. Une proportion moindre de sujets interrogés des deux sexes acceptent d'avoir affaire «aux deux». Enfin, c'est seulement comme « inférieures » que les femmes elles-mêmes préfèrent se trouver devant « des femmes », dans une proportion assez importante (41\%), mais qui n'atteint même pas la moitié des cas. Ce refus des femmes de la part des hommes et même d'une forte proportion des femmes peut être l'expression de la hiérarchie sociale, dans le travail, entre les deux sexes, à laquelle nous venons de faire allusion.

Activités que les femmes exercent mieux que les hommes. À côté de ces reproches, on reconnaît à la femme des capacités particulières pour l'exercice de certains métiers. Les hommes sont plus formels en ce sens que les femmes ${ }^{2}$. Il s'agit, pour celles-ci, soit d'une méconnaissance de leurs capacités (les «je ne sais pas » atteignent le quart

1 Voir à ce sujet Isambert-Jamati (V). « Les facteurs familiaux et professionnels de l'absentéisme féminin ». Dans : Rev. franç. Trav., 13,1-1959.

286,7 à $90 \%$ des hommes affirment, d'une façon plus ou moins nuancée, que les femmes exercent mieux qu'eux certains métiers, alors que 56,7 à 75\%, des femmes adoptent la même attitude. 
de leurs réponses), soit, plus rarement, d'un refus de distinguer les aptitudes propres à chaque sexe, et, par suite, la spécialisation des métiers.

Les activités où la femme réussit le mieux sont, d'après les sujets interrogés, les suivants : travaux minutieux, soins aux malades, soins aux enfants, travail social, métiers qui mettent en contact avec le public (vendeuses, serveuses, emplois de bureau, couture) [voir tableau 3].

Tableau 3.

Activités où la femme réussit le mieux (d'après les sujets interrogés).

\begin{tabular}{lcccc}
\hline Sujets interrogés & $\begin{array}{c}\text { Travaux } \\
\text { minutieux }\end{array}$ & $\begin{array}{c}\text { Soins aux } \\
\text { enfants, aux } \\
\text { malades, travail } \\
\text { social }\end{array}$ & Couture & $\begin{array}{c}\text { Vendeuse, serveuse, } \\
\text { métiers exigeant des } \\
\text { rapports avec la } \\
\text { clientèle, activités de } \\
\text { bureau }\end{array}$ \\
$\begin{array}{c}\text { Échantillon ouvrier } \\
\text { Hommes }\end{array}$ & $\%$ & $\%$ & $\%$ & $\%$ \\
Femmes & 15 & 15 & 15 & 20 \\
Échantillon intermédiaire & 15 & 11,7 & 6,7 \\
Hommes & 28,3 & 26,7 & 20 & 26,7 \\
Femmes & 5 & 23,3 & 10 & 10 \\
\hline
\end{tabular}

Il y a, pour ces choix, plus de points communs entre les individus du même sexe qu'entre ceux du même milieu, sauf pour les activités concernant les enfants et le travail social, qui sont préférés par les intermédiaires. La plupart des «travaux minutieux » mentionnés sont ceux qui s'effectuent en usine : bobinage, montage, électronique, etc. Le refus, si fréquent, de l'usine pour la femme serait alors surtout dû aux conditions dans lesquelles s'exerce le travail et à l'ambiance qui règne dans les ateliers.

Qualités qui rendent la femme plus apte. Les qualités qui rendent les femmes plus aptes à ces métiers sont décrites beaucoup plus par les hommes que par les femmes, qui, souvent, «ne savent pas » ou n'ont pas répondu. L'analyse de contenu fait apparaître un modèle féminin plus net chez les hommes que chez les intéressées ellesmêmes. Les femmes sont décrites comme « agiles, habiles, adroites, ayant du soin et du doigté, l'amour du détail... ». D'autres sont plutôt sensibles aux dons psychologiques qu'ils ont l'habitude de voir manifester à la femme dans sa vie familiale : "sa compréhension humaine, sa douceur, ses dons auprès des enfants... ». Enfin, quelques sujets interrogés insistent sur la qualité des relations avec autrui, non plus cette fois dans le sens du dévouement, mais dans le sens d'une certaine efficacité : " elle sait y faire, a bon contact avec le client, est aimable... ». Le fait que la femme exerce ou non une profession ne semble pas modifier son attitude, ni celle de son mari, sur ce point.

Métiers où l'on préfère l'homme ou la femme. Après avoir laissé les personnes interviewées décrire des types de métier et des qualités « féminins », nous leur avons 
proposé diverses professions que les deux sexes peuvent exercer en leur demandant s'ils préfèrent y rencontrer un homme ou une femme. La femme est bien acceptée dans les magasins, aux guichets des administrations et comme juge d'instruction. Elle est refusée comme directeur d'entreprise par les ouvriers, pour l'instruction des garçons par tous les sujets interrogés, comme médecin par les hommes, en ce qui les concerne ${ }^{1}$.

Nous avons ensuite demandé si l'on accepterait d'avoir affaire à des hommes dans des métiers habituellement féminins. Pour le service social, les avis sont très partagés; seules les femmes intermédiaires préfèrent un peu plus souvent un service social confié à des femmes. Dans les hôpitaux, les hommes envisageraient plus facilement d'être soignés par des hommes, les femmes le refuseraient davantage. Mais, à la maison, il est amusant de constater que ce sont les femmes qui refusent de voir l'homme effectuer les travaux ménagers. ${ }^{2}$ Elles craignent sans doute son immixtion dans un domaine qui leur est propre, comme les hommes refusent leur présence dans le monde de l'usine... Toutefois, lorsque la femme travaille, elle approuve davantage la participation du mari aux travaux domestiques. D'une part, elle a davantage besoin d'aide, d'autre part, les rôles sont alors moins différenciés, comme nous le verrons plus loin.

Inversement, on accepte assez mal la présence de femmes dans les métiers exercés jusqu'à ce jour presque uniquement par des hommes. Si les sujets interrogés pensent, en majorité, que la femme peut faire un bon chauffeur de taxi, sans doute parce qu'ils l'ont vue à l'œuvre depuis plusieurs années, ils ne l'imaginent ni ajusteur, ni électricien, ni mécanicien d'auto, ni pilote d'Air France. Il n'y a pas de différence entre sexes sur ces points. Les ouvriers sont plus opposés à la femme électricien, les intermédiaires à la femme pilote. Rappelons à ce propos qu'un nombre élevé d'intermédiaires travaillent à Air France, et connaissent bien le milieu de l'aviation. Certaines personnes de l'échantillon décrivent les réussites féminines dans l'une ou l'autre de ces professions, mais ne les considèrent guère autrement que comme des exceptions, et généralisent peu.

La réussite de la femme dans les professions non typiquement féminines est donc ressentie comme exceptionnelle. L'opposition règne en ce domaine à l'égard de la femme et lui rend d'ailleurs le succès difficile.

1 La question posée était : «Accepteriez-vous aussi facilement d'aller chez un médecin femme que chez un médecin homme?»

2 La différence sur ce point entre refus et acceptation selon les sexes est significative à .05. 


\section{Les préoccupations économiques et les aspirations réelles des femmes}

$\underline{\text { Retour à la table des matières sur la femme en France }}$

Motifs du travail féminin. Si le travail féminin soulève bien des questions de principe, il ne faut pas oublier qu'il est, avant tout, une nécessité, tout au moins dans les milieux défavorisés du point de vue économique. Les réponses spontanées des sujets interrogés vont presque toutes dans ce sens. La nécessité économique est donnée comme la cause du travail féminin dans la plupart des cas ${ }^{1}$.

Les femmes sont plus sensibles que les hommes aux questions financières, niais ce sont elles qui, la plupart du temps, tiennent le budget du ménage. Lorsqu'on présente aux sujets interrogés la liste de propositions suivante : «Quelles sont les principales raisons qui poussent les femmes à travailler pour améliorer le budget, pour faire des achats à tempérament, parce qu'elles s'ennuient à la maison, par goût et intérêt pour le métier, pour avoir de l'argent à soi, pour être indépendantes, pour avoir une retraite, pour permettre aux enfants de continuer leurs études, pour d'autres raisons ? », c'est le premier point qui est choisi par la quasi-totalité des intéressés. Les rubriques «indépendance et goût pour le métier »n'apparaissent presque pas dans l'échantillon ouvrier $(3,3 \%$, des hommes, $0 \%$ des femmes), mais sont assez souvent invoquées chez les intermédiaires (28 à $30 \%)$. Ces différences sont très significatives. Pour pouvoir exercer un métier qui lui plaise vraiment, la femme ne doit pas avoir un niveau socio-économique trop bas. Actuellement, la femme de milieu ouvrier, qui a une formation professionnelle souvent inexistante et travaille avec un bas rendement économique, est poussée par la nécessité plus que par le goût pour une profession.

La rubrique «faire continuer les études aux enfants » est également fréquemment choisie. Là aussi, l'idée de nécessité est présente ; les ouvriers la citent plus que les intermédiaires, mais aussi les femmes plus que les hommes, dans le même milieu ${ }^{2}$.

Les autres rubriques sont plus rarement choisies : l'ennui à la maison, par $10 \%$ des intermédiaires hommes et femmes et 6,7 et $1,6 \%$ respectivement des hommes et femmes de l'échantillon ouvrier.

Nécessité réelle et stéréotypes. Ces résultats appellent quelques commentaires. D'une part, il semble que certaines des femmes n'aient pas osé exprimer le goût pour le travail professionnel qu'elles ont manifesté par la suite, au cours du déroulement de l'enquête. La nécessité est sans doute le motif dominant pour elles, mais elles ont obéi

1 Parmi les ouvriers, 68,7\% des hommes et $86,7 \%$ des femmes estiment que le travail de la femme est dû à la nécessité économique; parmi les intermédiaires, 83,3\% et 98,3\%. respectivement.

2 Parmi les ouvriers, $50 \%$ des hommes et $70 \%$ des femmes; parmi les intermédiaires, $30 \%$ des hommes et $28,4 \%$ des femmes. 
aussi à une certaine contrainte de l'opinion, qui tend à présenter la mère au travail comme soumise à un devoir plutôt que satisfaite d'exercer un métier.

D'autre part, l'attitude hostile de certains des hommes à l'égard des femmes qui travaillent renforce cette hypothèse. Voici quelques réponses typiques en ce sens. Les raisons du travail sont, d'après ces hommes : «le vice, la toilette, l'argent mis de côté », « le goût de la recherche, le goût de l'aventure... », " si la femme va à l'usine, c'est pour faire la putain, 45 sur 50 le font ». Ces réponses sont rares ; mais, même si elles n'expriment que l'opinion d'une très faible minorité, elles suffisent à expliquer une réserve chez la femme.

Avantages et inconvénients de la solution choisie: contrainte économique et aspiration. Lorsqu'on demande aux mères les avantages et les inconvénients de la solution qu'elles ont adoptée - travailler ou rester au foyer - celles-ci mettent une fois de plus en avant la question financière, bien plus nettement que leurs maris, dans les deux milieux.

Celles qui travaillent parlent de l'amélioration du budget, celles qui restent au foyer déplorent «le manque d'argent», mais mettent en avant les économies ainsi réalisées. L'éducation des enfants et la tenue de la maison sont les thèmes qui apparaissent en seconde position. Le milieu intermédiaire (et surtout les femmes de ce milieu) s'exprime souvent dans les termes suivants : "vie agréable, plus riche, loisirs, etc. »- ou inversement : « vie limitée, ennui, etc. »- et cela aussi bien sur le plan familial que sur le plan personnel, quelle que soit la solution choisie. Les conditions de vie sont moins contraignantes pour les femmes de ce milieu que pour les hommes et les femmes de milieu ouvrier, qui jamais n'ont répondu de cette façon.

Quels sont donc les désirs des femmes interrogées dans le domaine professionnel : Travailler à domicile, au-dehors ou s'occuper uniquement de leur foyer ? La femme de milieu ouvrier préfère rester au foyer dans la grande majorité des cas, qu'elle y soit actuellement ou qu'elle travaille $(68,7$ à $73 \%)$. La solution vécue ne modifie donc pas le choix. Pour les femmes de milieu intermédiaire, il en va tout autrement. Lorsque la femme est au foyer, elle souhaite un peu moins y rester $(58,9 \%)$ que la femme de milieu ouvrier. Mais, lorsqu'elle travaille, elle ne quitterait son travail que dans $25 \%$ des cas.

Les femmes sont donc beaucoup plus satisfaites d'exercer un métier chez les intermédiaires qu'en milieu ouvrier. De même, en milieu ouvrier, les hommes estiment très fréquemment que la solution adoptée est « une bonne solution », lorsque la femme est au foyer $(73,3 \%$ et beaucoup plus rarement si la femme travaille $(36,8 \%$ En revanche, les intermédiaires approuvent presque toujours la formule choisie, que la femme reste chez elle (95\%) ou travaille $(75 \%)$

Quand la contrainte économique diminue, le libre choix devient possible et la satisfaction s'accroît, non seulement pour les intéressées, mais pour le couple.

L'importance du facteur économique se précise de plus en plus. Il nous a donc semblé indispensable de pousser plus avant l'analyse des préoccupations budgétaires et la manière dont se répartissent dans les couples les rôles en matière de gestion budgétaire. 
Les difficultés budgétaires. La plupart des couples ouvriers n'arrivent pas à «boucler leur budget » ou y arrivent «juste » $(86,7 \%)$. Un peu plus de la moitié $(56,7 \%)$ des intermédiaires éprouvent les mêmes difficultés. À première vue, les intermédiaires, qui ont des revenus plus élevés, ont Plus souvent des budgets en équilibre. Cependant, plusieurs d'entre eux sont issus de familles très modestes et ont progressé grâce à des efforts considérables. Ils ressentent alors le besoin d'avoir un foyer stable et bien équilibré pour soutenir la promotion du chef de famille. D'autre part, dans l'organisation de leur budget, la part des éléments culturels se fait de plus en plus lourde, d'autant plus qu'il s'agit de ménages en voie de promotion sociale. L'apparition de nouveaux besoins leur crée parfois des difficultés budgétaires.

La gestion du budget. Que la femme soit particulièrement attentive aux questions économiques au cours de l'enquête correspond simplement au fait que, dans la plupart des foyers ouvriers et dans beaucoup de foyers d'intermédiaires, c'est elle qui gère le budget. En milieu ouvrier, presque tous les hommes et les femmes approuvent, en principe, une gestion féminine du budget $(80$ à $76,7 \%)$; chez les intermédiaires, la moitié seulement des femmes et 56,7\% des hommes adoptent le même point de vue. Plus du tiers des femmes intermédiaires préfèrent que les deux membres du couple s'occupent ensemble de cette question, alors que les hommes pensent plutôt à prendre eux-mêmes cette question en charge quand ils ne la confient pas à leur femme. Cette tendance est indiquée déjà en milieu ouvrier, mais les différences entre les désirs de l'homme et ceux de la femme ne sont pas. significatives.

Si l'on détaille différents points du budget - grosses dépenses, dépenses courantes, impôts et assurances, loyers et charges, etc. - deux faits se dégagent avec certitude : $1^{\circ}$ la fréquence des sujets interrogés des deux sexes qui sont favorables à la gestion du budget par la femme est significativement (.01) plus élevée en milieu ouvrier ; $2^{\circ}$ parmi ceux qui envisagent une gestion autre que celle de la femme, la gestion par « les deux » membres du couple est souhaitée beaucoup plus par la femme, la gestion «par l'homme », par l'homme lui-même.

La femme, pour le moment, reste la grande responsable de l'économie domestique, surtout en milieu ouvrier. L'homme, en lui remettant son salaire, lui fait confiance et lui donne une grosse responsabilité, tandis que, dans d'autres milieux, la femme se plaint d'être obligée de demander de l'argent à son mari. Mais la gestion d'un budget insuffisant est une très lourde charge, dont l'homme se débarrasse trop aisément. Certaines femmes souhaiteraient davantage la participation de leur mari à cet égard. Le geste qui consiste à tout abandonner à la mère de famille peut être un geste de grande confiance et de partage des tâches de la part de l'homme, ou le geste du seigneur à l'égard de son intendant, ou encore une attitude de démission à l'égard d'une femme plus mûre et plus responsable que lui...

Les hommes des deux milieux ont exprimé à plusieurs reprises l'idée que «si la femme reste à la maison, c'est elle qui assure le budget ; sinon ils se partagent le travail ». La gestion du budget apparaît donc comme une tâche plus que comme un privilège. Certains des sujets interrogés reconnaissent que cette gestion exige des dons qui sont souvent reconnus à la femme, ou bien qu'on doit demander à « celui qui est le plus doué » d'assumer cette charge. Fréquemment, les dépenses courantes sont laissées à la femme, les impôts et les assurances à l'homme, les grosses dépenses incombant le plus souvent «aux deux » (environ 50\% des cas), moins souvent à la femme et encore plus rarement à l'homme. 
L'analyse des comportements des sujets interrogés et de leurs aspirations reflète certaines conceptions des rôles de l'homme et de la femme dans la société. Des questions faisant apparaître directement le terme d' «égalité » entre l'homme et la femme, ou y faisant appel indirectement, ont soulevé des réactions très vives et révélatrices. L'examen des changements d'attitude de la femme qui travaille et de son mari constitue une première approche de ce problème. Les divers plans sur lesquels les sujets interrogés situent cette égalité, la valeur qu'ils lui attribuent méritent une analyse aussi fine que possible. Nous n'exprimerons ici que les premiers résultats d'un travail de longue haleine.

\section{a) La société. La femme et le couple. Le principe de l'égalité des sexes}

$\underline{\text { Retour à la table des matières sur la femme en France }}$

Pour la femme mariée, le travail a été un moyen de s'émanciper. Impliquant des conditions bien souvent pénibles, surtout en milieu ouvrier, meilleures dans d'autres milieux, le travail amène des transformations des rôles traditionnels dans la famille. Mais les bouleversements qui se produisent au niveau des structures sociales ne sont pas sans créer des malaises et des inquiétudes.

Travail de la femme, avantages ou non pour la société. Aussi, les deux tiers des réponses dans les deux échantillons indiquent-elles que le travail de la femme mariée est un mal pour la société ${ }^{1}$. Les femmes, elles, estiment, nettement plus souvent que les hommes, que «c'est un bien » (.05). Les arguments qui justifient ces affirmations sont, d'une part, économiques, d'autre part, relatifs à la vie familiale. En milieu ouvrier, on craint que la femme ne prenne la place des hommes, qu'elle ne soit une source de chômage. Chez les intermédiaires, on voit, dans le travail des femmes mariées, la preuve «que les payes sont de moins en moins grandes pour les maris ». Du point de vue familial, on craint que la vie des foyers n'en pâtisse, « on n'a plus assez de temps pour l'éducation des enfants », «il y a plus de divorces ». En somme, «c'est un mal pour la société car cela casse la famille et chacun sait que la vie familiale, c'est la base de la société ». Quand le travail des femmes mariées est jugé bénéfique pour la société ${ }^{2}$ c'est seulement pour des raisons économiques : «il y a davantage d'argent qui circule ». Un groupe moins important adopte une position plus nuancée : «cela dépend », ou « c'est indifférent ${ }^{3}$.

Travail de la femme et avantages pour elle-même. Pour la femme mariée ellemême, le travail est encore jugé un mal par $60 \%$, des sujets interrogés et un bien par $23 \%$ seulement. Mais, à l'intérieur des échantillons, et selon les sexes, les résultats sont différents. Nous retrouvons ici une progression que nous avions signalée plus haut : $31,7 \%$ des femmes de l'échantillon intermédiaire, $25 \%$ de celles du milieu

\footnotetext{
La question posée à ce sujet était : «Certaines personnes remarquent qu'un ensemble de plus en plus grand de femmes mariées sont obligées de travailler. Est-ce un bien, est-ce un mal pour la société, pour la femme elle-même, pour l'égalité avec les hommes ?»

2 De 13 à $23 \%$. des hommes et femmes interrogés.

3 De 8 à 16\%, selon les sexes et les échantillons. Pas de différence significative.
} 
ouvrier, 18,3\% des hommes de l'échantillon intermédiaire et enfin 17,7\% seulement des ouvriers jugent que le travail est « un bien » Pour la femme mariée elle-même. La position «c'est un mal » varie en sens inverse. Les autres positions sont moins nombreuses (8,3 à 18,3\%). Elles dominent chez les femmes de l'échantillon intermédiaire et décroissent suivant la même progression que l'opinion «c'est un bien ». Souvent l'idée de nécessité apparaît, accompagnée d'un refus de jugement de valeur.

Des commentaires justifient les choix. Les sujets interrogés qui voient dans l'exercice d'un métier « un bien » pour la femme mariée sont favorables à son indépendance économique à l'égard de son mari et à son ouverture d'esprit : "ça les dégourdit», «elles sont au courant de tout», « une femme qui reste chez elle n'évolue pas ».

Les commentaires qui expliquent l'attitude «c'est un mal » portent soit sur la mauvaise tenue du foyer, soit sur la fatigue (ces deux idées sont surtout exprimées en milieu ouvrier), soit encore sur le risque que court la femme de subir de «mauvaises influences », de «mauvais exemples », de « devenir vulgaire », de « se trouver parmi des personnes qui n'ont pas la même culture... une promiscuité morale s'établit ». Toutes ces remarques ont été formulées par des hommes des deux échantillons. Ils considèrent la femme comme un être particulièrement influençable.

Enfin, une dernière attitude révèle la crainte que l'émancipation trop grande de la femme ne crée la mésentente au sein de la famille. La femme «n'est plus si féminine », « est moins attachée à son foyer », « elle ne joue plus son rôle de mère ». Les réflexions traduisent les diverses conceptions qui ont cours touchant les rôles attribués à la femme. La question relative à l'influence du travail sur l'égalité avec l'homme conduit à des réponses encore plus significatives.

Le travail et l'égalité avec l'homme. L'influence du travail sur l'égalité avec l'homme est reconnue par $63 \%$ des ouvriers et $68 \%$ des intermédiaires. Cette fois encore, les femmes de l'échantillon intermédiaire $(75 \%)$ et, un peu moins nettement (65\%), celles de l'échantillon ouvrier sont plus disposées à admettre cette influence que les hommes (61\% dans les deux échantillons). Parmi ceux, peu nombreux, qui ne croient pas à une modification de l'égalité par le travail, trois positions se distinguent. Pour les uns, « hommes et femmes sont égaux, la femme n'a pas besoin de travailler pour cela »; d'autres confondent et refusent les termes d'égalité et d'identité, et insistent sur les différences et la complémentarité des sexes. Enfin, un troisième groupe soutient l'infériorité de la femme par rapport à l'homme, en s'appuyant sur des raisons psycho-physiologiques : «les femmes ont moins de résistance, sont plus soumises à leurs nerfs », etc.

Lorsque l'influence est jugée bonne (60\% des femmes et 51,6\% des hommes de l'échantillon intermédiaire, 50 et $40 \%$ des femmes et hommes de l'échantillon ouvrier), c'est souvent parce que « le travail apporte à la femme un rajustement de sa condition », conçue comme subalterne. L'homme estimerait davantage la femme lorsqu'il lui reconnaît des capacités dans le domaine de la production et peut la mettre sur le même plan que lui : «Il y a trop d'hommes qui ont tendance à croire la femme inférieure... ça leur fait du bien de voir une femme bien réussir. »

Une idée proche de la précédente, mais plus fréquemment exprimée par l'échantillon ouvrier, c'est que la femme accède alors à une véritable dignité humaine et «ne se sentira plus dans une position inférieure », « elle ne sera plus alors contrainte par 
l'homme aux besognes dures et à effectuer seule tous les travaux du ménage ». Ces transformations se répercutent sur la vie au sein du foyer. Les ouvriers, surtout, soulignent que «cela leur permet de revendiquer le droit à la parole pour bien des choses» et que « la femme a évolué en travaillant, le mari était trop maître chez lui ».

Un domaine où cette influence du travail est soulignée est celui de l'argent. Les femmes qui travaillent risquent moins les reproches de leurs maris en ce qui concerne les dépenses du ménage, surtout selon les sujets interrogés dans l'échantillon ouvrier. Les intermédiaires ont une idée plus nette des progrès ainsi réalisés au sein du couple : l'homme et la femme accèdent entre eux à des rapports plus vrais, plus directs et à plus de compréhension.

La tendance à l'égalité des sexes est considérée comme novice par 17 à $20 \%$ des sujets interrogés, mais beaucoup plus dans le quartier de type ancien (27\%) que dans celui de type nouveau $(12,5 \%)$. Les quartiers de type nouveau regroupent des populations appartenant aux branches professionnelles les plus dynamiques et des couples plus jeunes et plus féconds, nous l'avons déjà souligné ${ }^{1}$. C'est aussi là que ces nouvelles formes de couple se dessinent le plus.

Les motifs exprimés pour justifier les craintes à l'égard de l'égalité entre les sexes sont de plusieurs ordres. Ils peuvent, toutefois, se résumer dans l'inquiétude que la disparition d'une hiérarchie établie dans la société et la famille ne se solde par du désordre et par des souffrances pour les deux sexes.

Certains pensent que, si la femme se sent égale, elle voudra souvent « dominer dans le foyer, commander ». Les femmes, bien plus que les hommes, libèrent souvent, à l'occasion de cette question (et d'autres concernant des sujets voisins), une certaine agressivité, qui apparemment se retourne contre elles-mêmes. Elles ne peuvent alors s'imaginer auprès de leur mari, sans manifester un désir de commander à leur tour. L'égalité serait, en conséquence, source de mésentente dans le ménage. Deux postulats paraissent se dégager de cette assertion, d'après des commentaires variés : d'une part, dans le couple un équilibre est impossible à réaliser, l'un cherche toujours à « faire la loi » à l'autre. D'autre part, c'est à l'homme de commander, «c'est l'homme qui doit être au-dessus, c'est naturel ». L'homme, mis en face d'une femme qui travaille et devient par là égale à lui, « est diminué, et ne se sent plus chef de famille », « une femme ne doit pas prétendre gagner comme son mari ».

Pour d'autres, la nécessité est due au fait que la femme «n'a pas besoin d'être toujours sur un pied d'égalité avec l'homme, chacun a son rôle, elle n'a pas besoin d'être égale à l'homme, elle a un autre but, celui d'assurer la marche de son foyer, qui est sa véritable fonction $»$.

Le désir de maintenir une hiérarchie entre les sexes se révèle pour une fraction assez importante des sujets interrogés, mais celui de différencier les rôles est encore bien plus souvent exprimé au cours de l'enquête, et pas seulement dans les réponses aux questions relatives au travail professionnel ${ }^{2}$.

Le niveau d'instruction scolaire des deux membres du couple. Des attitudes très proches des précédentes ont pu être dégagées de questions portant sur l'instruction

Voir p. 31. [du numéro XIV de la revue]. Voir dans cette édition réf page 31.

Voir p. 35. [du numéro XIV de la revue]. Voir dans cette édition réf. page 35. 
scolaire. Les points de vue y sont exprimés avec des nuances affectives marquées. La question était formulée dans les termes suivants : "Vaut-il mieux que la femme ait une instruction moins poussée, plus poussée, ou égale à celle de son mari ? » Elle faisait suite à une question plus générale sur l'importance de l'instruction scolaire pour la vie du couple, qui avait permis aux sujets interrogés des deux échantillons d'exprimer leur grand désir d'instruction.

Plus de la moitié des sujets interviewés des deux échantillons souhaitent que les deux membres du couple possèdent un même niveau d'instruction. Les intermédiaires répondent souvent aussi qu'ils préfèrent, pour la femme, une instruction «pas plus poussée que celle de son mari »- formule que nous avons préférée exprimer telle quelle plutôt que de classer les réponses dans les rubriques «égale » ou « moins poussée » (voir tableau 4)

Tableau 4. Niveau d'instruction pour la femme.

\begin{tabular}{|c|c|c|c|c|c|c|}
\hline \multirow[b]{2}{*}{$\begin{array}{l}\text { Sujets } \\
\text { interrogés }\end{array}$} & \multicolumn{6}{|c|}{ Instruction } \\
\hline & $\begin{array}{l}\text { Moins poussée } \\
\text { que celle du mari }\end{array}$ & Égale & $\begin{array}{l}\text { Pas plus } \\
\text { poussée }\end{array}$ & $\begin{array}{l}\text { Plus } \\
\text { poussée }\end{array}$ & $\begin{array}{c}\text { Pas } \\
\text { d'importance }\end{array}$ & $\begin{array}{l}\text { Pas de } \\
\text { réponse }\end{array}$ \\
\hline \multicolumn{7}{|l|}{ Ouvriers } \\
\hline Hommes & 5 & 63,3 & - & 13,3 & 13,4 & 5 \\
\hline Femmes & 35,3 & 70 & - & 0 & 6,6 & 1,6 \\
\hline \multicolumn{7}{|l|}{ Intermédiaires } \\
\hline \multirow[t]{2}{*}{ Hommes } & 18,3 & 56,7 & 10 & 10 & 15 & 3,3 \\
\hline & \multicolumn{6}{|c|}{66,7} \\
\hline \multirow[t]{2}{*}{ Femmes } & 25 & 51,7 & 20 & 1,6 & - & - \\
\hline & \multicolumn{6}{|c|}{71,7} \\
\hline
\end{tabular}

Si la majorité des hommes et des femmes accordent leurs préférences au même niveau d'instruction, les femmes déclarent assez souvent qu'un niveau inférieur est préférable et n'envisagent pratiquement pas la possibilité d'un niveau supérieur. Certains des maris, eux, accepteraient cette dernière éventualité.

Les résultats quantitatifs se passent de commentaires, mais l'analyse de contenu permet d'atteindre le niveau des motivations des attitudes. Le mari voit l'aspect utilitaire de l'instruction de sa femme, pour la vie du foyer et pour lui-même : « la femme peut avoir une instruction plus poussée, pour pouvoir éduquer les enfants » (ouvrier, homme); «elle peut s'occuper des enfants et donner des cours au mari » (ouvrier, homme) ; cela peut servir au mari, à condition de le faire d'une façon intelligente » (intermédiaire, homme).

La femme pense davantage aux rapports interpersonnels. Elle imagine, ou elle a peut-être constaté, une attitude revendicative ou agressive chez une femme qui, sur ce plan, est supérieure à son mari : « une instruction un peu moins poussée que celle de son mari, ce n'est pas mauvais pour une femme, il ne faut pas qu'elle gagne des points » (ouvrier, femme) ; «dans certains cas, quand la femme est plus instruite, elle peut faire sa maligne » (ouvrier, femme) ; «s'il y a égalité, ils ont des discussions; 
quand l'instruction est plus poussée, le mari se sent inférieur » (ouvrier, femme); «ça le vexerait » (intermédiaires, deux femmes); «elle voudrait prendre la tête du ménage » (intermédiaire, femme).

La crainte des femmes est probablement le signe d'un certain sentiment d'infériorité ; elles se méfient de leurs propres réactions, qui pourraient être hostiles, lorsque la possibilité d'être supérieures sur un point dans le couple leur est donnée. Une femme interrogée, qui elle-même travaille, nous a surpris en déclarant : « la femme doit avoir une instruction inférieure, puisque c'est l'homme qui travaille ». Mais une autre juge la situation avec humour : « tout ça peut très bien s'accorder, mais on peut laisser à l'homme le plus, puisqu'il y tient ».

Bien que deux tiers des couples environ prennent position en faveur d'une même instruction scolaire dans le couple, nous avons insisté sur les choix extrêmes qui reflètent des courants de pensée particuliers, mettent à jour certaines difficultés entre les sexes et expliquent d'autres attitudes, à l'égard du travail par exemple. Rappelons ici qu'à la question «Le travail professionnel modifie-t-il la manière d'agir de la femme à l'égard du mari ? » la grande majorité des sujets interrogés avaient déclaré qu'effectivement la femme changeait et que c'était un mal. Par principe, on refuse difficilement l'égalité de l'homme et de la femme (sauf quand le terme est assimilé à identité), mais, au niveau des situations vécues, l'indépendance et l'affirmation de la femme sont souvent mal supportées. Peut-être y a-t-il une crainte que le mouvement amorcé n'aille trop loin ? Ou encore a-t-on l'image de couples difficiles, de certains excès ? Seule l'étude de la perception de la manière dont la condition de la femme a évolué peut permettre, dans une certaine mesure, de répondre à ces questions.

\section{La perception de l'évolution du statut de la femme en fonction des changements des structures sociales et des conditions de vie}

$\underline{\text { Retour à la table des matières sur la femme en France }}$

Les changements du statut de la femme dans la société, que les sujets interrogés critiquent ou approuvent, vont de pair avec des transformations dans les structures de la société. Quelles transformations juridiques ou sociales ont le plus frappé les deux milieux étudiés ici ? Nous avons essayé, au cours de cette enquête, de saisir la façon dont une évolution est perçue par des populations bien définies.

\section{a) Les changements des droits}

D'après les trois quarts des sujets interrogés, les droits de la femme se sont réellement accrus depuis la guerre de 1939-1945, Sur ce point, les hommes sont encore plus affirmatifs que les femmes ( $85 \%$ contre $74 \%)$, mais les deux échantillons ne diffèrent pas. Les droits évoqués se situent soit sur le plan juridique, soit sur le plan 
de la vie quotidienne, familiale ou professionnelle, et consacrent un nouvel état de fait.

Les milieux ouvriers sont moins sensibles à l'accès au bénéfice des «nouvelles lois sociales » que décrivent les intermédiaires, et surtout à l'accès à de nouvelles responsabilités professionnelles. Mais la plus grande indépendance des femmes dans la vie quotidienne les frappe vivement.

Si les sujets interrogés perçoivent une évolution, ils envisagent ce problème de façon différente. Les uns le centrent autour d'une évolution générale tendant à l'égalisation des sexes, d'autres recherchent quels sont, en fait, les droits légaux acquis. Les premiers pensent que la femme se libère sur le plan des mœurs et devient plus indépendante d'une façon générale. Le mouvement progresse, d'après certains, d'une façon passive, inéluctable; d'après d'autres, il se fait en fonction des transformations de la femme, qui lui viennent soit d'une éducation différente, soit de circonstances exceptionnelles, grâce auxquelles elle a pu se manifester (la guerre par exemple). Les seconds décrivent les droits acquis : droit de vote, droit d'ouvrir un compte en banque, droit au salaire égal, diminution des emplois réservés aux hommes, etc.

Un groupe de sujets interrogés estime que les droits sont obtenus théoriquement, mais ne sont pas passés dans les mœurs. Enfin, quelques-uns nient toute évolution, soit que l'acquis soit insuffisant depuis 1939 pour qu'il mérite d'être mentionné, soit que les femmes aient « toujours pris leurs libertés quand elles en avaient envie ».

Cette évolution des droits et de la liberté de la femme aboutit-elle à une égalité des droits des deux sexes ? Cette fois, les sujets interrogés sont moins massivement affirmatifs : les gens de l'échantillon intermédiaire moins que les ouvriers, les femmes moins que les hommes, les habitants du quartier nouveau un peu moins que ceux du quartier ancien. En somme, ceux qui, tout au cours de l'enquête, se sont révélés le plus désireux de voir la condition de la femme évoluer vers une plus grande libération et vers davantage d'égalité avec l'homme pensent, moins fréquemment que les autres, que cette égalité est déjà réalisée dans les structures actuelles. Leur niveau d'aspiration est plus élevé sur ce point.

\section{b) L'ouverture de nouveaux débouchés pour les femmes}

$\underline{\text { Retour à la table des matières sur la femme en France }}$

Les récentes conquêtes de nouveaux droits, de nouveaux débouchés par la femme peuvent amener certaines transformations de la société. Plus de la moitié des personnes interviewées $(52 \%)$ croient que des changements se produiront. Cette fois encore, les femmes de l'échantillon intermédiaire pressentent cette évolution plus que les autres sujets interrogés.

Les modifications envisagées portent sur le domaine professionnel, pour l'homme et la femme, sur l'économie du pays, sur le plan social, sur la vie familiale ou encore sur l'ensemble des conditions de vie de la femme. Les ouvriers insistent avant tout sur les changements dans l'économie : avec un accroissement du pouvoir d'achat, une 
amélioration du commerce, « cela apportera le bien-être ». En revanche, sur le plan professionnel, la crainte d'une concurrence entraînant du chômage s'est fait sentir à plusieurs reprises.

Dans les deux échantillons, l'idée que les femmes ont un rôle particulier à jouer sur le plan social et politique a été souvent exprimée : "Au point de vue social, les femmes discutent et elles ont meilleure vue » (échantillon ouvrier, femme, treizième arrondissement); "Pour les questions familiales, il faudrait des femmes au gouvernement » (ouvrier, homme, treizième); «Elles participent plus à la vie sociale; le point de vue des femmes est généralement assez différent, pour un bon nombre de problèmes, de celui des hommes. Cela devra être pris en considération. Par exemple, sans la femme, on ne peut faire des progrès essentiels dans les problèmes d'éducation » (intermédiaire, homme, Villejuif); «Les femmes peuvent apporter plus de douceur dans les rapports humains » (intermédiaire, femme, treizième). Ce nouveau rôle attribué à la femme compense la difficulté que les foyers auront à recevoir : «On recevra de moins en moins, il n'y aura plus de contact entre amis. La vie sociale perdra de son charme » (intermédiaire, femme, treizième). Les échanges se feraient alors davantage à l'occasion du travail professionnel, et l'ambiance générale de la société en bénéficierait.

Ceux qui souhaitent une évolution espèrent que, dans l'avenir, cette évolution se répercutera sur la vie familiale : "On finira par admettre que la femme n'est pas un être inférieur » (intermédiaire, femme, Villejuif); «La femme ne sera plus astreinte à être la servante de toute la famille, elle aura un rôle plus important dans la société. Cela changera la structure de la famille » (intermédiaire, homme, treizième); et «Il y aura une plus grande compréhension entre l'homme et la femme » (intermédiaire, femme, treizième). D'autres, désirant conserver à la femme son rôle traditionnel, expriment leurs craintes devant les perspectives d'une société nouvelle : «Les femmes n'auront plus le rôle qui leur est imposé par la nature, c'est-à-dire le rôle de mère, au sens moral du mot » (intermédiaire, homme, treizième) ; «Cela diminuera l'intimité familiale » (intermédiaire, femme, treizième); «Ce serait un mal qu'elle dirige, elle est à sa place maintenant » (intermédiaire, homme, treizième) ; " Plus une femme prend de l'autorité, plus elle a tendance à renoncer à son rôle » (intermédiaire, femme, treizième) ; «Elle pourra dire "je suis autant que toi", cela fait des difficultés dans le ménage » (ouvrier, homme, Villejuif).

La femme elle-même aura « une vie plus indépendante »; « elle aura plus d'ouverture d'esprit »; « ayant des postes plus importants, elles changeront de mentalité ».

Certains sujets interrogés, beaucoup moins nombreux, ne voient pas la société changer $(3,2 \%)$, soit qu'il leur semble que les transformations sont déjà accomplies et ne se poursuivent plus, soit que l'évolution leur apparaisse comme une chose complexe, que l'ouverture de nouveaux débouchés aux femmes ne peut expliquer, ou encore qu'ils refusent toute évolution. Un intermédiaire de Villejuif renforce son affirmation en ce sens : "D'ailleurs, on leur a donné trop de fonctions depuis la Libération. On n'aurait pas dû les faire voter. La femme a ses devoirs, ses charges, l'homme a les siens. La femme doit rester bien définie dans son rôle intérieur. Elle a un rôle de maîtresse de maison » (intermédiaire, homme, Villejuif).

Il est intéressant de comparer cette position de principe stricte et traditionnelle aux aspirations d'une femme de Villejuif, qui décrit une société transformée; il y aurait : «Un mélange étroit des deux sexes, une plus grande liberté dans les mœurs. Cela 
pourrait être un bien, dans la mesure où l'amitié et la solidarité joueraient. C'est peutêtre un peu utopique. On se sent peut-être mieux exister en tant qu'homme et en tant que femme en présence d'individus de l'autre sexe ».

À l'occasion de cette question, les sujets interrogés ont projeté leurs aspirations et certaines de leurs préoccupations. Ainsi, les ouvriers qui ne voient pas de changements dans la société laissent percer parfois un ton un peu désabusé dans leurs commentaires : «Avant que la femme vote, cela n'allait pas bien, maintenant cela ne va pas mieux »; «Cela ne pourra rien changer »; «Cela sera exactement pareil »; «Il y a des années que la femme travaille, cela n'a rien changé ».

D'autres enfin se sont sentis dépassés par la question et n'ont pas su répondre (surtout des femmes de milieu ouvrier : $18 \%$ ).

L'observation de la manière dont les gens perçoivent l'évolution de la condition de la femme sur d'autres plans -plan de la vie familiale, plan du dialogue des sexes - peut aider à mieux comprendre leurs aspirations fondamentales.

\section{c) Les changements dans la vie familiale}

\section{$\underline{\text { Retour à la table des matières sur la femme en France }}$}

La vie familiale est naturellement la première à subir (en bien ou en mal, selon les opinions) les répercussions de ces transformations de la société. Il y a, à la fois, des changements de conditions de vie et des changements d'attitudes. Souvent les sujets interrogés disent qu'ils n'ont plus les mêmes préoccupations.

Changements de préoccupations d'une génération à l'autre. Du point de vue familial, $70 \%$ des ouvriers estiment avoir les mêmes préoccupations que leurs parents; 48,3\% seulement des intermédiaires adoptent la même attitude. Une différence aussi marquée peut tenir au fait que les intermédiaires sont en pleine promotion sociale. Un certain nombre d'entre eux sont d'origine ouvrière. Le fait aussi que, dans le groupe des intermédiaires de Villejuif, on ne rencontre que 35\% de sujets interrogés qui se sentent, de ce point de vue, proches de leurs parents est particulièrement significatif Rappelons, en effet, que c'est dans ce groupe que nous avions trouvé la plus grande fréquence d'hommes travaillant dans les branches professionnelles les plus jeunes et les plus dynamiques. En revanche, en milieu ouvrier, l'appartenance à l'un ou l'autre quartier n'apporte aucun changement d'attitude quant aux préoccupations familiales.

«Autres préoccupations » ne signifie pas moindres préoccupations dans l'esprit des sujets interrogés. Certains les rapportent uniquement à leur situation familiale et surtout au nombre de leurs enfants. Certains ont moins de soucis que leurs parents parce qu'ils ont peu d'enfants et sont issus de familles très nombreuses; pour d'autres, c'est le contraire.

D'autres évoquent le travail professionnel de la femme et la vie au foyer de sa mère, ou, à peu près aussi souvent, le fait contraire. Chez les intermédiaires, on trouve un peu plus souvent des réflexions sur les difficultés de la vie telles que : «la vie plus 
difficile », «plus de soucis, de préoccupations ». Une femme exprime peut-être assez bien l'attitude de quelques intermédiaires : «J'ai plus de préoccupations, plus de mal, ou plus d'ambition. » Certains d'entre eux sont, en effet, en pleine ascension sociale.

Quelques-uns, enfin, indiquent des différences portant sur des considérations générales, sans émettre de jugements de valeur : "c'est différent, le genre de vie n'était pas le même », «mes parents vivaient à la campagne », " cela s'est modernisé », « ce que maman disait est vrai, mais ce n'est plus la même vie », " elle s'occupait davantage de son intérieur, donnait de l'importance à des choses qui me sont égales », « on ne voit plus la vie comme mes parents la voyaient, on a une optique différente, il y a une évolution des mœurs ».

Transformation des activités familiales d'une génération à l'autre. Si l'évolution des préoccupations est perçue par une proportion assez importante des sujets interrogés, la transformation de la façon dont s'opèrent les tâches domestiques est sans doute plus facile encore à saisir. Il s'agit, en effet, cette fois de remarques portant directement sur des comportements.

Pour l'entretien de la maison, plus de la moitié de l'échantillon ouvrier $(54,2 \%)$, quel que soit le sexe, ne remarque pas de différence entre la manière de procéder des femmes de cette génération ou de la génération précédente. L'amélioration des méthodes n'est soulignée que par $31,7 \%$ des membres de cet échantillon qui font allusion soit à l'utilisation d'appareils ménagers, soit au fait de la présence au foyer de la femme «qui fait le ménage tous les jours », alors que «sa mère travaillait» ou « était aux champs », soit encore au fait que la famille comprend moins d'enfants. La position des hommes de l'échantillon intermédiaire est voisine de celle des hommes du milieu ouvrier, mais ils remarquent encore moins le progrès en ce domaine $(23,3 \%$ seulement). En revanche, 43,3\% des femmes de l'échantillon intermédiaire voient une amélioration dans la manière dont elles effectuent les tâches ménagères. Cette amélioration est d'ailleurs encore plus souvent notée à Villejuif que dans le treizième arrondissement, non seulement pour les femmes du milieu intermédiaire (46,7\%), mais aussi pour celles du milieu ouvrier.

Lorsque les familles s'installent dans des logements de type nouveau, elles ont tendance à acheter des appareils ménagers, ce qui peut, en partie, expliquer les remarques des femmes. Les différences entre les deux milieux peuvent avoir pour cause la plus grande facilité des femmes du milieu intermédiaire à acquérir ces appareils, en raison de leur meilleure situation économique. Certaines familles intermédiaires sont d'origine ouvrière et ont des conditions de vie plus faciles que celles de leurs parents et elles se sentent souvent «plus évoluées », tant sur le plan matériel que sur le plan culturel. Cette disposition d'esprit se retrouve constamment au cours de l'enquête et aide le chercheur à dégager la perception de l'évolution, tant du point de vue des comportements que de celui des attitudes.

Hommes et femmes n'ont pas la même attitude, quel que soit le milieu cette fois, quand il s'agit de l'éducation des enfants. La proportion des hommes qui estiment les méthodes employées par leurs femmes et leurs mères identiques est double de celle des hommes qui trouvent meilleures les méthodes appliquées par leurs femmes. En revanche, une proportion beaucoup plus élevée de femmes ont l'impression d'avoir de meilleures méthodes que leurs mères : autant cette fois que de femmes imitant consciemment la génération précédente. 
Les progrès porteraient surtout sur la souplesse de l'éducation, ou sur la meilleure compréhension des enfants : "L'éducation est plus souple, l'autorité ne s'exerce plus de la même façon » (femme de Villejuif) ; « À mon avis, je le fais (exercer l'autorité) sur des bases plus saines. On est plus préoccupé de ce que peuvent penser les enfants, de ce qu'on peut faire pour les aider » (femme de Villejuif); «Il me semble qu'on a une compréhension qu'on n'avait pas avant » (femme du treizième). Les améliorations concerneraient aussi les soins, l'instruction plus poussée. Enfin, certains réagissent en s'opposant à la génération précédente : «On essaie de ne pas faire les erreurs de ma famille »; « on n'empoisonne pas les enfants avec des choses secondaires »; «il y a plus d'entente entre nos enfants et nous; ça fait une grande différence avec les parents ».

Quelques personnes $(8,3 \%)$ regrettent la manière d'agir plus ferme et autoritaire de leurs parents : «Ma femme n'est pas assez sévère » (ouvrier, homme, Villejuif); «Ma mère était plus sévère que moi » (intermédiaire, femme, Villejuif) ; «De mon temps, il n'y avait pas de zazous, on était tenus ». Globalement, la moitié de l'échantillon perçoit un changement dans les méthodes éducatives - changement qui est le plus souvent décrit comme bénéfique.

Les divergences entre sexes, sur ce point, peuvent sans doute être attribuées au fait que les femmes ont eu plus souvent des difficultés avec leurs mères que leurs maris (les conflits mères-filles sont bien connus) - d'où une attitude d'opposition plus forte de leur part. Elles peuvent aussi tenir à ce que le mari s'occupe moins directement de ses enfants, et juge de plus loin la façon de procéder de sa femme.

A côté de ces diverses tâches familiales, nous avons essayé de savoir si une évolution dans les loisirs était également perçue. Ici encore, les sujets interrogés marquent un progrès. En effet, le quart seulement des membres des échantillons ne croient pas à un changement, alors que les modifications qui sont décrites vont presque toutes dans le sens «du mieux ». Comme pour beaucoup des attitudes analysées jusqu'ici, ces améliorations sont ressenties légèrement plus par les intermédiaires que par les ouvriers et plus par les femmes que par les hommes ${ }^{1}$. Pour certains, ce ne sont pas les types de loisirs qui rapprochent les générations, mais plutôt le manque de loisirs qui est le lot commun des femmes : « je n'en ai pas », déclarent plusieurs femmes de l'échantillon ouvrier du treizième arrondissement; «Les loisirs sont maigres, ce sont les promenades du dimanche, tant que les enfants sont petits » (homme de Villejuif). Les loisirs décrits sont surtout la télévision, le cinéma et les vacances.

Les loisirs parfois ne peuvent se comparer, car la situation du couple interviewé est trop différente de celle des parents : par exemple, quand l'origine rurale de la famille est proche ou que le nombre d'enfants est très élevé. Quelques personnes trouvent que le rythme de vie rapide d'aujourd'hui ne laisse pas de place pour des loisirs. En somme, comme le déclare un sujet interrogé : « Nos mères avaient plus de temps, mais on utilise plus facilement le temps maintenant. »

Le regret du « bon vieux temps » est, dans l'ensemble, assez rare, et les conditions pénibles de la vie des femmes des générations précédentes sont beaucoup plus souvent évoquées.

Amélioration des loisirs : échantillon ouvrier, hommes, $35 \%$; échantillon intermédiaire hommes, $43,3 \%$; ouvrier, femmes, $48,3 \%$ intermédiaire,femmes, $51,7 \%$. 
Les modèles de référence. Cette croyance au progrès est-elle à la base de l'attitude des femmes qui, lorsqu'elles ont une difficulté à résoudre à propos de leurs enfants ou de leur maison, essaient de « se débrouiller seules » (65\% des cas), ou avec leur mari $(10,8 \%)$, mais se réfèrent peu à leur mère $(8,3 \%)$ ou à leur belle-mère $(4,1 \%)$ ? Une telle attitude peut être le signe d'un simple besoin de s'affirmer, de se sentir adulte à l'égard des parents et de prendre ses responsabilités. Mais elle peut traduire aussi la coupure de deux générations et le désir de rejeter les modèles anciens.

L'attachement au passé. Pourtant, l'attachement des femmes au passé et, en particulier, à la famille d'origine est affirmé par les sujets interrogés : 51,6\% des hommes et $70 \%$ des femmes estiment l'attachement au passé plus marqué chez la femme que chez l'homme. Les premières seraient «plus sentimentales », " elles aiment à s'accrocher au passé », « à leurs souvenirs, à leur jeunesse », « elles se souviennent, les hommes oublient vite ». "L'homme vit plus au jour le jour, la femme est plus romanesque, elle pense à sa vie de jeune fille »; «c'est l'instinct conservateur de la femme ».

Les femmes concevraient cet attachement sous un aspect très sentimental : souvenirs de famille, d'enfance, de jeunesse, rappel du temps des fiançailles, de la vie de jeunes mariés, etc. Cela n'est probablement pas en contradiction avec le désir d'une évolution favorisant l'émancipation de la femme, comme certaines l'ont exprimé, ou, au moins, améliorant les conditions de vie. Ainsi pense un homme de l'échantillon intermédiaire, séparant les différents plans : «Si l'on parle du passé familial, la femme est plus attachée au passé. Du point de vue social, elle est moins traditionaliste. » Une femme du même milieu est encore plus explicite : «Les femmes mettent plus de temps à se débarrasser des idées reçues, mais elles ont plus d'audace après, tandis que les hommes sont très attachés à la forme de vie et à la société qui les avantagent. Même ceux qui ont des idées très avancées n'arrivent pas à renoncer à leurs anciens privilèges.»

Cette vision de la femme attachée sentimentalement au passé, ou peut-être surtout à son passé personnel et à la jeunesse et, en même temps, souvent consciente d'être plus libre et d'effectuer ses travaux ménagers ou d'éduquer ses enfants mieux que la femme de la génération précédente, correspond sans doute à une situation exacte. Néanmoins, il est probable que ce lien affectif avec le passé s'accompagne de certains modèles dont la femme n'a pas toujours conscience, mais qui peuvent la guider. D'un autre côté, des, souhaits de transformation des structures et de progrès ont été émis par bien des femmes. Des conflits éclatent facilement entre de tels attachements et de telles aspirations.

L'évolution de la vie familiale qui est perçue ou souhaitée pour l'avenir a été envisagée jusqu'ici sur le plan des activités générales. Les transformations des relations entre les personnes et, principalement, entre l'homme et la femme dans la famille et le couple n'ont pas encore été abordées. Elles nous semblaient si essentielles que nous avons évité de les traiter avant d'avoir mis au point l'évolution de certaines structures sociales et de la vie familiale. Cependant, il n'est pas douteux que l'évolution de ces relations est à la fois conséquence et cause des transformations des structures sociales. 


\section{La perception des changements de comportement des deux sexes à l'égard l'un de l'autre}

$\underline{\text { Retour à la table des matières sur la femme en France }}$

Les modifications du comportement de la femme à l'égard de l'homme depuis vingt ans frappent la majorité des sujets interrogés (64\%). Les changements de l'homme à l'égard de la femme sont moins remarqués (48\%). Plusieurs variables (sexe, âge et milieu social) modifient ces résultats.

\section{a) La perception des changements en fonction du sexe, de l'âge et du milieu social, d'origine et actuel}

Les hommes ont à peu près aussi souvent les mêmes attitudes dans l'échantillon ouvrier et dans l'échantillon intermédiaire, tandis que les femmes ont une perception très différente de la situation en milieu ouvrier et en milieu intermédiaire. En effet, celles de l'échantillon ouvrier trouvent beaucoup moins souvent que celles de l'échantillon intermédiaire que les comportements des deux sexes se sont transformés (voir tableau 5)

Plusieurs explications peuvent être données à ces résultats. Si les femmes de l'échantillon intermédiaire ressentent si souvent les modifications de comportement, c'est ou bien parce qu'elles aspirent très vivement à la transformation des structures sociales et sont attentives aux moindres changements, ou bien parce que les comportements des deux sexes ont réellement évolué.

En milieu ouvrier, si les femmes sont moins sensibles aux modifications des comportements, ce peut être soit parce qu'elles sont écrasées par leurs conditions de vie et que «c'est toujours pareil », soit parce que des changements se sont produits en milieu ouvrier avant cette période de vingt ans, c'est-à-dire avant la dernière guerre. Les années 1930 ont correspondu à des prises de conscience de la classe ouvrière et à un grand dynamisme qui n'ont pas leur équivalent dans la période qui a suivi les grèves de 1950-1951. En revanche, les petites classes moyennes, surtout dans le milieu des techniciens, semblent davantage en essor aujourd'hui. Une troisième hypothèse pourrait être, au contraire, que l'émancipation de la femme est moins souvent réalisée en milieu ouvrier que dans les classes moyennes. La réflexion d'une ouvrière va dans ce sens : "L'homme n'a pas changé, il aime bien se croire le maître toujours, même si ce n'est qu'une illusion! Avant, c'était pareil. » Les relations entre les sexes 
paraissent, en effet, à certains soumises à des lois immuables, les modifications dues au milieu étant de faible portée.

\section{Tableau 5}

\begin{tabular}{|c|c|c|c|}
\hline Questions posées et sujets interrogés & Oui & Non & $\begin{array}{c}\text { Ne sait } \\
\text { pas }\end{array}$ \\
\hline \multicolumn{4}{|c|}{ La femme a-t-elle changé depuis vingt ans à l'égard de l'homme? } \\
\hline \multicolumn{4}{|l|}{ Échantillon ouvrier } \\
\hline Homme & 53,3 & 25 & 21,7 \\
\hline Femme & 65 & 18,3 & 16,7 \\
\hline \multicolumn{4}{|l|}{ Échantillon intermédiaire } \\
\hline Homme & 78,3 & 13,3 & 8,4 \\
\hline Femme & 60 & 23,3 & 16,7 \\
\hline \multicolumn{4}{|c|}{ L'homme a-t-il changé depuis vingt ans à l'égard de la femme? } \\
\hline \multicolumn{4}{|l|}{ Échantillon ouvrier } \\
\hline Homme & 36,7 & 40 & 23,3 \\
\hline Femme & 45 & 36,7 & 18,3 \\
\hline \multicolumn{4}{|l|}{ Échantillon intermédiaire } \\
\hline Homme & 66,7 & 23,3 & 10 \\
\hline Femme & 46,7 & 41,7 & 11,6 \\
\hline
\end{tabular}

L'analyse des différences de réponses en fonction d'autres variables peut renforcer l'une ou l'autre de ces hypothèses.

La variable "âge» et les changements de l'homme. La perception de cette évolution est également liée à la génération puisque, dans un même milieu, les deux sexes semblent trouver moins de transformations quand ils dépassent l'âge de 40 ans ${ }^{1}$. S'agit-il alors d'une moindre possibilité d'évoluer ou de voir évoluer ? (Certains, les plus âgés, ne peuvent s'abstraire de leur vie de couple qui n'a pas changé.) S'agit-il du fait que les modifications apparentes sont sans grand poids par rapport aux échanges fondamentaux entre les sexes auxquels plusieurs se réfèrent ? Ou bien se trouve-ton devant un phénomène sociologique, une modification des structures sociales, des comportements et des aspirations, après cette guerre ? Il est bien difficile de trancher en faveur d'une de ces hypothèses.

Les changements de la femme. Les transformations du comportement de la femme sont plus évidentes que celles de l'homme, dont le rôle a moins changé depuis vingt

1 Avant 40 ans, $11 \%$ seulement des femmes trouvent que l'homme n'a pas changé; après 40 ans, $50 \%$. Les proportions, pour les hommes, varient du tiers, avant 40 ans, aux deux tiers après. 
ans. Ici les sujets interrogés ont remarqué l'évolution de la femme dans une proportion beaucoup plus forte que celle de l'homme. Cependant, cette fois encore, ce sont les personnes les plus âgées qui croient le moins à cette évolution ${ }^{1}$.

Comme cela était prévisible, ce sont les femmes de milieu ouvrier nées avant 1919 que les changements frappent le moins et, inversement, les femmes du milieu intermédiaire âgées de moins de 40 ans qui y sont le plus sensibles.

L'origine des parents. La perception d'une évolution pourrait correspondre à un simple fait objectif : le changement de classe sociale des sujets interrogés qui, ainsi, évoqueraient simplement des transformations de façons de vivre. Cette hypothèse a dû être infirmée. En effet, ce sont les sujets interrogés dont le milieu socioprofessionnel d'appartenance et le milieu d'origine sont identiques qui remarquent le plus une évolution. D'une part, le déroulement de l'existence dans un même milieu Permet peut-être de mieux saisir les transformations qui s'y produisent. D'autre part, les comparaisons entre milieux laissent sans doute transparaître des constantes fondamentales dans les comportements entre les sexes, auprès desquelles les nouvelles manières d'agir apparaissent comme peu importantes.

Nous l'avons indiqué à plusieurs reprises : les intermédiaires perçoivent davantage l'évolution. Ce sont donc, ici, les intermédiaires issus du milieu intermédiaire qui, par exemple, estiment le plus que l'homme a changé à l'égard de la femme. Les intermédiaires issus d'autres milieux viennent ensuite, puis les ouvriers dont les parents étaient ouvriers, enfin les ouvriers appartenant à d'autres milieux d'origine. Que les intermédiaires soient issus d'employés ou d'ouvriers, cela n'entraîne pas de différence de perception. En revanche, nous devons faire une place à part aux ouvriers dont les parents appartenaient au milieu intermédiaire. Il s'agit alors de petits intermédiaires, artisans ou contremaîtres; ce groupe ne comprend malheureusement que 13 cas. (Voir tableau 6.)

Tableau 6.

Perception de l'attitude des deux sexes l'un envers l'autre.

\begin{tabular}{|c|c|c|c|c|c|c|c|c|c|}
\hline \multirow{4}{*}{ Appartenance } & \multicolumn{9}{|c|}{ Origine } \\
\hline & \multicolumn{3}{|c|}{ Milieu ouvrier } & \multicolumn{3}{|c|}{ Milieu intermédiaire } & \multicolumn{3}{|c|}{ Autre milieu } \\
\hline & Oui & Non & $\begin{array}{l}\mathrm{Ne} \\
\text { sait }\end{array}$ & Oui & Non & $\begin{array}{l}\mathrm{Ne} \\
\text { sait }\end{array}$ & Oui & Non & $\begin{array}{l}\mathrm{Ne} \\
\text { sait }\end{array}$ \\
\hline & $\%$ & $\%$ & $\begin{array}{c}\text { pas } \\
\%\end{array}$ & $\%$ & $\%$ & $\begin{array}{c}\text { pas } \\
\%\end{array}$ & $\%$ & $\%$ & $\begin{array}{c}\text { pas } \\
\%\end{array}$ \\
\hline Échantillon ouvrier & 48,2 & 31,4 & 20,4 & 53,8 & 23 & - & 34,9 & 43,9 & 21,2 \\
\hline Échantillon intermédiaire (1) & - & - & - & 54,4 & 26,9 & 7,7 & 50 & 35,3 & 14,7 \\
\hline
\end{tabular}

1. Des femmes de l'échantillon intermédiaire, d'origine intermédiaire, $79 \%$ ont répondu « oui ».

1 Un tiers des personnes nées avant 1919 ne pensent pas que la femme ait changé, point de vue que partagent seulement $15 \%$ de celles qui sont nées depuis. 
Or 3 sujets seulement sur ces 13 ne trouvent pas de changements dans le comportement de l'homme. Il semble donc que cette perception dépende de l'information et du milieu culturel. Le milieu d'appartenance prédomine, mais, lorsque le milieu d'origine est mieux informé, plus cultivé que le milieu d'appartenance, il continue à exercer son influence sur l'adulte qui l'a quitté.

Le sexe intervient encore une fois dans le sens présumé : $79 \%$ des femmes de l'échantillon intermédiaire et d'origine intermédiaire décrivent des modifications de comportement. Ce pourcentage atteint $84 \%$ si l'on ne tient compte, dans ce même groupe de femmes, que de celles qui habitent dans la cité nouvelle de Villejuif. En cumulant les effets des variables qui favorisent la perception de l'évolution, on arrive à sélectionner des groupes où la quasi-totalité des sujets perçoivent une situation dans une perspective prévisible. Évidemment, d'autres variables interviennent et les fluctuations individuelles empêcheront presque toujours d'atteindre les $100 \%$

\section{La nature de cette évolution}

$\underline{\text { Retour à la table des matières sur la femme en France }}$

Comment a changé la femme. Ceux qui ont constaté une évolution du comportement féminin la situent, avant tout, sur le plan de l'émancipation, de l'indépendance. Non seulement la femme est «moins craintive » à l'égard de l'homme, elle n'est plus « le petit chien docile d'autrefois », mais « elle se laisse moins faire », « elle a plus d'assurance », «plus d'autorité », « elle n'accepte plus tout sans rien dire et se défend mieux ». Ces types de réponses spontanées à la question ouverte "Comment la femme a-t-elle changé à l'égard de l'homme ? » sont très fréquentes; elles représentent 70 des réponses des femmes de l'échantillon intermédiaire et de 25 à 36 de celles des hommes et femmes des autres milieux. Dans le même ordre d'idées, les réponses évoquant l'égalité avec l'homme et une plus grande liberté à son égard sont aussi exprimées fréquemment ( 28 à $50 \%$ des sujets interrogés qui ont remarqué des changements). Parfois même des sujets interrogés évoquent les transformations du couple : «Elle épaule davantage son mari, l'homme est moins seul », approuve un intermédiaire de Villejuif, alors qu'une femme du même milieu, tout en constatant cette égalité, regrette, semble-t-il, un peu la vie plus sentimentale de sa belle-famille et le style «camarade » de son ménage : «Ma belle-mère s'entendait bien avec son mari, c'était un grand amour ; mais vraiment le mari était le chef, presque le maître. Nous, ce n'est pas pareil, on est plus camarades, plus ensemble. » D'autres sujets interrogés décrivent des changements relatifs à la mode : «elle est moins élégante », ou au contraire « elle est plus coquette », « plus difficile pour s'habiller ».

Les critiques franches à l'égard des nouveaux comportements féminins sont peu fréquentes ( 9 cas) ; elles portent soit sur la légèreté, liée à l'acquisition de la liberté « elle est plus volage », « elle n'est pas toujours émancipée pour le bon », «ce n'est pas un bien, cette liberté, elle leur fait prendre une allure libre »- soit sur l'égalité des rôles avec l'homme : elle devient «moins féminine, elle veut être trop égale à 
l'homme, ce qui est néfaste », ou « elle ramène la paie à la maison, d'où discussions », «elle aime moins son mari ». Enfin, deux hommes craignent les répercussions sur la famille de cet état de fait. Ils estiment la femme «moins obéissante dans la famille » et « la famille, c'est moins sérieux qu'avant». Toutes ces critiques, excepté une, émanent de sujets de sexe masculin.

Les changements des comportements de la femme à l'égard de l'homme sont attribués au travail, à la guerre et aux «événements », aux nouveaux droits acquis, à la « vie moderne » en général, à l'instruction identique des individus des deux sexes, et, exceptionnellement, à la formation politique des femmes. La cause qui, de loin, est le plus souvent invoquée reste le travail et le fait que, par là, la femme contribue au gain et se sent indépendante : «elle dit : - je gagne assez seule » et « elle se sent son égale, elle peut apporter autant d'argent que lui ».

Comment a changé l'homme. L'homme s'est adapté aux nouveaux comportements et aux nouveaux rôles de la femme. «En général, il a accepté l'égalité » et les sujets interrogés des deux sexes jugent l'homme «plus tolérant». Il ne considère plus la femme " comme une bonniche », « comme une esclave », " comme une servante », il « prend la femme comme confidente », «il la considère comme une compagne », « une camarade ». Le travail de la femme est, là aussi, une des causes de cette transformation du comportement. Il a pour conséquence, pour l'homme, la nécessité d'apporter sa contribution au travail domestique. Mais, si J'acceptation de l'égalité avec la femme est reconnue par la moitié des femmes des deux échantillons, un peu plus des intermédiaires du sexe masculin et un peu moins des ouvriers, cette aide à la femme n'est que peu signalée ${ }^{1}$.

Près du quart des sujets interrogés tempèrent leur satisfaction des progrès réalisés sur le plan de l'égalité par certaines critiques des attitudes masculines. L'homme aurait perdu « sa courtoisie », il serait devenu «moins prévenant», « moins galant», « moins attentionné », « moins respectueux », « moins chevaleresque ». Ces remarques émanent des deux sexes. Un ouvrier trouve l'homme «plus mufle. Puisqu'elles ont les mêmes droits (disent-ils), dans le métro, je garde ma place ». Une femme se plaint de la démission de l'homme qui « devient plus insouciant à l'égard de la femme et ne prend plus ses responsabilités ». D'autres croient déceler une certaine jalousie et une rivalité de la part de l'homme, qui considère la femme comme une concurrente dans le travail.

L'évolution qui tend à rapprocher les deux sexes et à abolir les distances qui les séparent n'est pas sans atteindre aussi certains privilèges. D'une part, l'homme, qui se sent un peu détrôné, peut, du même coup, abdiquer toute responsabilité. D'autre part, la femme presque l'égale de l'homme, sans avoir tous ses avantages, n'en reçoit plus protection et hommage, mais peut se sentir camarade ou rivale. Or la galanterie, si elle est un signe de déférence qui s'est perdu avec J'avènement d'une plus grande égalité entre les sexes, a aussi tout un contenu sexuel et sentimental.

Est-ce que les sujets interrogés ne regrettent pas un peu cette forme du dialogue des sexes et ne craignent pas de le voir se ternir ? La crainte de certaines femmes en constatant l'évolution des sexes se réfère parfois à des principes, parfois elle peut être le signe de leur peur, devant des structures nouvelles, de perdre des avantages acquis,

1 Chez les hommes (échantillons ouvrier et intermédiaire), 7 et 11\%. Chez les femmes (échantillons ouvrier et intermédiaire), 17 et $18 \%$. 
alors que le gain n'apparaît pas toujours tellement appréciable, du moins dans les conditions d'existence actuelles. Mais surtout la vie sentimentale semble, à première vue, souffrir un peu du nouvel état de fait.

Les modèles de couple que présente soit la littérature soit le cinéma sont ou bien des modèles semblant vivre un amour authentique dans un style ancien, ou bien des modèles d'hommes et de femmes très libres, mais désabusés et dont l'amour semble voué à l'échec. La vision de couples formés d'hommes et de femmes bien intégrés dans la société et dont la position sociale libre n'entrave pas la vie sentimentale serait fort utile à présenter au public des salles de cinéma et aux lecteurs.

Il n'est pas normal que des femmes aient à choisir entre leur réalisation personnelle dans la société et leur vie de couple. Mais le choix que font la plupart d'entre elles est conditionné par la vision qu'elles ont du « bonheur».

\section{a) Les conséquences. La femme est-elle plus heureuse aujourd'hui ?}

$\underline{\text { Retour à la table des matières sur la femme en France }}$

L'évolution constatée sur des plans différents se solde par un résultat d'ensemble positif, puisque environ les deux tiers des sujets interrogés estiment que la femme « est plus heureuse aujourd'hui qu'autrefois ». Ni le milieu socio-professionnel, ni le sexe ne font varier cette proportion, seuls les intermédiaires masculins semblent un peu moins optimistes et ont tendance à nuancer un peu plus leur « oui ». Chez eux, le pourcentage moindre de « oui » absolus est compensé par un plus grand nombre de « oui, peut-être ».

Ces restrictions portent soit sur le milieu social de la femme («cela dépend des milieux », « oui, mais cela dépend de leur richesse »), soit sur le plan ou l'on peut situer le bonheur. Ces positions ${ }^{1}$ n'ont pas été comptées avec les « oui » absolus ; elles comportent toujours l'idée que, sur un plan ou sur un autre, il y a des femmes plus heureuses aujourd'hui qu'autrefois. Si l'on totalise les oui absolus et les oui relatifs, 75 à $80 \%$ des sujets interrogés trouvent la femme plus heureuse, au moins à certains égards.

Les causes de ce plus grand bonheur le plus souvent évoquées sont avant tout des améliorations des conditions de vie : « la vie est plus facile», «le travail est facilité », « il y a plus de commodités », «plus de progrès », «plus de confort». «Elles ont moins de peine, sont moins fatiguées grâce aux machines, aux frigidaires. » Le tiers des femmes - mais seulement un dixième des hommes-mettent en avant ces avantages dont elles bénéficient. Ce progrès semble lié à l'accroissement du pouvoir d'achat (augmentation des salaires) et aussi à certaines mesures sociales : congés payés, allocations familiales. Le fait qu'elles soient moins obligées de travailler est considéré aussi par quelques femmes comme un progrès. L'ambiance générale paraît, pour

1 De l'échantillon ouvrier, 6,6 à 13,3\%, et 20 à 26,6\% de l'échantillon intermédiaire adoptent cette attitude. 
certains, «plus gaie », il y a « des choses plus belles », « des couleurs extérieures plus claires ». Le cadre extérieur plus coloré contribuerait ainsi à donner de l'optimisme. Enfin, les loisirs seraient plus accessibles : «plus de toilettes, de spectacles », « autrefois, elles avaient moins de distractions, elles étaient plus esclaves de leur foyer ». Enfin, elles ont moins d'enfants et restent jeunes plus longtemps.

Une moindre proportion des sujets interrogés ${ }^{1}$ situe le bonheur de la femme dans son émancipation. Elle serait plus heureuse parce qu'elle est plus libre et mieux considérée : «elles ont davantage leur mot à dire », « elles sont plus libres, moins asservies », «elles obtiennent un peu plus de considération de la part de l'homme », « elles sont moins esclaves », « plus ça va, plus elles sont les égales de l'homme et ont la vie facile à la maison ».

Dans l'ensemble, les femmes seraient donc plus heureuses parce qu'elles ont des conditions de vie générales moins pénibles, plus de liberté, et qu'elles peuvent profiter des loisirs qui s'offrent à elles. La possibilité de se consacrer à un métier ou à une tâche prenante n'est pas évoquée, pas plus que la joie de former avec un mari un couple plus uni. On pense à une femme libérée des tâches ménagères trop dures, des charges professionnelles et de la soumission au mari. Les seules images positives qui se présentent sont les loisirs ou l'amélioration du cadre matériel. Le niveau d'aspiration des populations étudiées est-il très limité ou l'enquête n'a-t-elle abordé qu'un premier niveau?

Dans le questionnaire, la question était sans doute trop rapprochée de celles qui portaient sur l'amélioration des conditions de vie, mais c'est probablement davantage par des études de cas en profondeur qu'il serait possible d'aller plus loin. Enfin, ces résultats peuvent être aussi l'expression d'un grand besoin de détente chez les sujets interrogés.

Les motifs du petit groupe de ceux qui trouvent les femmes moins heureuses correspondent, en grande partie, au négatif des précédents : "autrefois, la femme pouvait rester chez elle, alors que maintenant elle est obligée de travailler », " elles ont moins de temps », " elles disposent peut-être davantage de confort, mais c'est un argent gagné à force de travail », " la vie est plus chère », " autrefois, on ne manquait de rien ». Non seulement on constate un surcroît de travail dans la vie moderne, mais on conteste les avantages acquis par l'augmentation des gains. « Actuellement, on est plus tenté par les choses nouvelles, donc il faut plus d'argent », " maintenant, on se crée plus d'exigences », « autrefois, on se contentait de moins », « la vie actuelle est plus compliquée », « les femmes étaient plus simples, plus heureuses », « on a plus de soucis », « plus de préoccupations », « le modernisme est une illusion ». Les difficultés budgétaires, le manque de temps et de détente et les préoccupations sont les motifs évoqués le plus souvent ici. Seul un homme pense à l'unité du couple : «Les ménages étaient plus unis, on s'entendait mieux. »

Un groupe de sujets interrogés, des intermédiaires ( 5 hommes et 1 femme), se sont dégagés des conditions matérielles, mais ne précisent alors pas ce qu'est le bonheur, qui apparaît parfois comme quelque chose de très individuel, parfois comme une sorte de fatalité : "C'est tellement relatif, tout ça, le bonheur », « le bonheur est une chose variable », « chacun fait son bonheur comme il l'entend », « je crois que la

1 De $12 \%$ (échantillon ouvrier) à 18\%. (échantillon intermédiaire). 
question bonheur n'a rien à voir avec les questions matérielles », «les femmes sont plus heureuses matériellement, mais, sur le plan moral, on est impuissant ».

Une contradiction apparaît ici dans les résultats, qui a déjà été signalée à d'autres reprises : le travail professionnel semble « un malheur » pour la femme, et cela non seulement dans les motifs décrits, mais même au niveau des faits. En effet, les femmes qui restent au foyer, ainsi que leurs maris, trouvent beaucoup plus souvent que la femme est maintenant plus heureuse que les couples où la femme exerce une profession. Or une autre source de bonheur pour la femme est sa plus grande liberté, la considération de l'homme, qui ont été acquises surtout en travaillant ${ }^{1}$. Le travail n'aurait-il comme but essentiel que de transformer le statut des femmes dans la société ? Est-ce que celles-ci l'abandonneront dès qu'elles auront obtenu des positions réellement égales à celles de l'homme ? Ou bien plutôt le travail des femmes est-il simplement, pour le moment, encore trop pénible pour beaucoup d'entre elles pour être accepté avec satisfaction ? C'est alors un bouleversement de la société qui s'opère et celles qui œuvrent pour l'émancipation de la femme, consciemment ou non, n'en sont pas toujours les bénéficiaires. N'oublions pas non plus que bien des conquêtes féminines ont été faites à l'occasion de guerres, où les femmes ont été amenées à prendre des responsabilités dans des conditions tragiques. Actuellement, la société est mal préparée à vivre ce changement de statut féminin.

\section{Conclusion}

$\underline{\text { Retour à la table des matières sur la femme en France }}$

Au terme de cette première étude, certaines conclusions se dégagent nettement, dont voici les principales :

La différence des points de vue suivant le sexe. La femme est généralement favorable à ce qui peut accélérer son émancipation, tout en se méfiant parfois de ses propres réactions agressives, lorsqu'elle se trouve sur un plan d'égalité avec l'homme et surtout sur un plan de supériorité. Elle reste tiraillée entre des aspirations différentes. Elle juge souvent en fonction de son problème personnel, tandis que l'homme voit moins l'intérêt de sa femme elle-même que celui du groupe familial.

La différence de point de vue suivant la catégorie socio-professionnelle. Apparemment les représentants des classes moyennes ont une vision des rôles féminins moins traditionnelle que celle des ouvriers. Mais ces derniers expriment souvent non des aspirations vraies, mais des réactions de défense en fonction de conditions de vie écrasantes. Ils recherchent le meilleur équilibre familial possible, avant d'essayer des formules nouvelles. Au-dessous d'un certain niveau économique, il n'y a pas de choix libre pour la femme. En milieu ouvrier, si elle est mariée et a des enfants, toute activité professionnelle ou militante hors du foyer est bien souvent une charge écrasante ou un luxe irréalisable. D'où la réaction des ouvriers contre les activités

1 Voir p. 59 [de l'édition papier de la revue]. Voir dans l'édition numérique. 
extérieures des mères de famille - réaction qui englobe parfois, d'un même mouvement, la transformation des rôles familiaux. Le modèle idéal est d'ailleurs plus d'une fois en contradiction avec la situation vécue.

Dans les petites classes moyennes, la femme trouve plus aisément la possibilité d'avoir des activités autres que les seules tâches ménagères et les rôles familiaux se transforment. L'homme participe davantage à la vie de la famille : rôle éducatif accru, aide aux travaux domestiques, etc. Une image de la femme plus libre peut donc être appréciée dans ces milieux.

L'influence des conditions d'habitat. La transplantation dans des cités nouvelles, du fait, sans doute, du dépaysement et des nouvelles possibilités offertes, rend les habitants plus sensibles à l'évolution de la situation de la femme et plus favorables à son émancipation. Complémentairement, l'implantation de longue date dans un quartier de type ancien fait obstacle à l'apparition de modèles nouveaux.

L'influence d'autres éléments (âge ou milieu d'origine par exemple). D'une manière générale, diverses images de la condition de la femme se présentent aux sujets interrogés qui portent des jugements de valeur sur elles. Nous connaissons actuellement les facteurs qui sensibilisent la perception de groupes déterminés à telle ou telle image. Nous pouvons ainsi mieux comprendre les aspirations de ces groupes en fonction de certaines conditions de vie et de courants culturels. La reconnaissance d'une évolution, le désir ou la crainte qu'elle se réalise sont des moteurs de la transformation de la société. Le rôle du psycho-sociologue est de découvrir ces moteurs, de les analyser et d'en faire prendre conscience aux intéressés eux-mêmes. 


\section{II \\ Les attitudes à l'égard de la condition de la femme dans les familles ouvrières polonaises}

Les deux articles polonais qui sont présentés ici se complètent pour rendre compte de l'enquête comparative qui a été menée à Lodz, à Varsovie et en Silésie. Mlle A. Kloskowska donne un aperçu du problème général de l'étude des images et des modèles et développe, à partir de données concrètes, des remarques suggestives sur les définitions et l'étude théorique des notions de base. M. Piotrowski centre son article sur les attitudes à l'égard du travail des femmes, qui est l'un des points les plus importants de l'enquête, dans l'état actuel de son déroulement.

I. Antonina Kloskowska, "Généralités. Attitudes à l'égard des rôles respectifs et de l'égalité des deux sexes et à l'égard des enfants"

II. J. Piotrowski, “Attitudes à l'égard du travail des femmes” [Université de Varsovie]

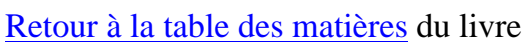


II

Les attitudes à l'égard de la condition de la femme dans les familles ouvrières polonaises

\section{I \\ "Généralités. Attitudes à l'égard des rôles respectifs et de l'égalité des deux sexes et à l'égard des enfants" par Antonina Kloskowska}

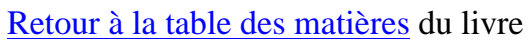

Table des matières : $1^{\text {er }}$ article sur les femmes en Pologne

Introduction

1. Résultats de l'enquête
a) Description de l'échantillon
b) Les conditions de vie et la gestion du budget
c) L'égalité de l'homme et de la femme et les modèles familiaux
d) L'avenir des enfants

2. Considérations théoriques concernant les recherches comparatives sur les modèles $\underline{\text { sociaux }}$ 


\section{Introduction}

$\underline{\text { Retour à la table des matières du } 1^{\mathrm{er}} \text { article sur les Polonaises }}$

Quand, à la fin de la première guerre mondiale, Florian Znaniecki, dans l'introduction au Polish peasant, décrivait la famille paysanne de son pays, cette description était valable pour la majorité des familles polonaises ${ }^{1}$. La Pologne, qui avait recouvré son indépendance politique le 11 novembre 1918, se transformait, dans la période située entre les deux guerres, en un pays agricole. En 1921, la population urbaine ne représentait que $24 \%$ de la population totale ; en 1931 , le pourcentage était de $27 \%$. Le progrès de l'urbanisation était donc lent. La crise économique pesait lourdement sur la population rurale et les traits essentiels de l'organisation traditionnelle de la famille paysanne, liée à une économie agricole assez primitive, devaient demeurer inchangés jusqu'au début de la seconde guerre mondiale.

Cette famille, dépeinte par F. Znaniecki en 1918, et étudiée de nouveau par Joseph Chalasinski en $1938^{2}$, était attachée, par des liens étroits, à l'ensemble du village ; elle se caractérisait par la soumission de ses membres à l'institution familiale considérée comme un tout. Dirigée, dans ses fonctions économiques et sociales, par le père, elle représentait le type de la petite famille patriarcale et laissait peu de place à l'individualité de ses membres. Elle ne favorisait pas l'expression des sentiments : les relations entre les enfants et les parents étaient empreintes de formalisme, de même que les relations entre l'homme et la femme ; celle-ci occupait dans le « modèle » de la famille une place subordonnée ${ }^{3}$.

Malgré les efforts isolés des institutions nationales et des organisations régionales d'adultes et de jeunesse rurale, la distance qui séparait la vie des paysans de celle des autres couches sociales restait très grande. Des différences marquées existaient entre la famille paysanne et la famille de l'intelligentsia, qui présentait toutes les caractéristiques de la famille moderne urbaine, où la femme était complètement émancipée et où les enfants jouissaient d'une large indépendance à l'égard de leurs parents.

Les changements économiques et sociaux d'après guerre ont ébranlé la structure agricole du pays. Entre 1946 et 1958, plus de quatre millions d'habitants des campa-

1 W. I. Thomas ; F. Znaniecki. Polish peasant in Europe and America, 1re édition, New York, 19181920.

2 J. Chalasinski. Mode pokolenie chlopow (La jeune génération des paysans).Warszawa, 1938, 4 vol. Cette oeuvre capitale, conçue dans le cadre de la méthodologie sociologique de l'école de Znaniecki, est basée sur 1500 autobiographies de jeunes paysans.

3 J'ai essayé de préciser la signification des termes «modèle » et «pattern » dans l'article «Changing family models in the popular magazines in Poland », dans Recherches sur la famille, vol. III (Éd. Nels Anderson, Gôttingen, 1958). 
gnes ont émigré vers les villes. La population de grandes villes, comme Wroclaw, à l'ouest de la Pologne, est constituée, dans une proportion de $80 \%$, par des gens venus des petites villes de province et des villages. La population urbaine représente actuellement plus de $46 \%$ de la population totale du pays. Le taux de nuptialité est plus élevé dans les villes qu'à la campagne. Pendant plusieurs années, le taux d'accroissement naturel dans les villes a égalé celui des campagnes et le nombre absolu des naissances a été sensiblement égal dans les villes et les campagnes ${ }^{1}$. C'est la famille urbaine qui représentera désormais le type dominant de la famille polonaise. Le progrès général de l'industrialisation et de l'urbanisation exerce aussi une influence sur la famille paysanne, mai il place la famille ouvrière au centre même de l'intérêt.

L'étude de la famille ouvrière polonaise, qui a été organisée dans le cadre de la recherche comparative présentée plus haut par P. Chombart de Lauwe, porte sur deux groupes de familles habitant deux des plus grandes villes polonaises : Varsovie, la capitale (1 900000 habitants), et Lodz, centre d'industrie textile (700 000 habitants) ${ }^{2}$.

\section{Résultats de l'enquête}

\section{a) Description de l'échantillon}

$\underline{\text { Retour à la table des matières du } 1^{\mathrm{er}} \text { article sur les Polonaises }}$

Le groupe de Lodz est composé de102 ménages; les noms des maris ont été tirés au sort sur la liste des bénéficiaires d'allocations familiales d'une usine textile (laine). L'échantillon peut être considéré comme représentatif des familles d'ouvriers mariés, ayant au moins un enfant d'âge scolaire ou préscolaire, domiciliés à Lodz et employés dans l'usine choisie. Cent ménages de Varsovie représentent les familles d'ouvriers de l'industrie métallurgique travaillant dans des établissements divers.

Selon les principes de sélection, l'âge des maris est compris entre 25 et 60 ans ; à Varsovie, $30 \%$ des maris ont plus de 45 ans, à Lodz, 26,5\% ont dépassé cet âge. Ni Varsovie, ni Lodz n'ont été atteintes par la vague principale d'immigration rurale, qui était dirigée plutôt vers les nouveaux centres industriels et vers les villes de l'Ouest. Mais elles n'ont pas été exclues non plus du processus général d'infiltration campa-

1 En 1931, le taux de nuptialité, en Pologne, était le suivant (pour 1000 habitants) : villes, 7,9; campagne, 8,7 ; en 1956, le taux était de 10,2 pour les villes et de 8,7 pour la campagne. En 1931, le total des naissances était de 183800 pour les villes et de 765800 pour les campagnes ; en 1958, de 328300 pour les villes et de 427200 pour les campagnes. (Annuaire Statistique, 1958 et 1959.)

2 Les recherches, à Varsovie, ont été conduites par la Section sociologique de l'Institut d'économie nationale, sous la direction de M. Georges Piotrowski; M. Piotrowski et son groupe de recherche ont aussi participé à l'élaboration finale de la version polonaise du questionnaire et ont dépouillé les résultats de leurs recherches. 
gnarde, d'autant que, même avant la guerre, la campagne jouait le rôle de réservoir de la classe ouvrière polonaise.

En ce qui concerne les 102 ménages ouvriers de Lodz, 38\%, des hommes et 37\% des femmes sont nés à la campagne, et y ont même habité, au moins jusqu'à leur seizième année ; à Varsovie, les proportions correspondantes sont moins élevées ; toutefois elles dépassent le quart de l'échantillon.

Nos deux échantillons représentent deux branches d'industrie différentes en ce qui concerne les conditions de travail les salaires, et les qualifications moyennes des ouvriers. Les ouvriers du groupe varsovien sont, en général, mieux rémunérés, leurs conditions de logement sont meilleures, leur niveau d'instruction et leur qualification professionnelle sont plus élevés.

Les familles de Lodz comptent, en tout, 217 enfants, Soit 2,12 par famille ; celles de Varsovie, 225 enfants, soit 2,25 par famille. La différence la plus importante entre les deux groupes, du point de vue de cette étude, a trait à la participation des femmes au travail professionnel. Dans l'échantillon de Lodz, 67,64\% des femmes sont employées à l'extérieur, contre $35 \%$ seulement dans l'échantillon de Varsovie. Ces pourcentages sont beaucoup plus élevés, en ce qui concerne les femmes mariées exerçant une profession, que ceux qui sont indiqués dans une étude basée sur les données du recensement de $1931: 31,8 \%$ à Lodz et 16,3\% à Varsovie ; on remarquera, cependant, que le rapport existant entre les deux pourcentages est sensiblement le même dans les deux cas.

Le problème du travail salarié des femmes et des attitudes à l'égard de ce travail à l'intérieur des familles ouvrières polonaises sera traité séparément dans un autre article. Le présent article est consacré à d'autres aspects des opinions et des attitudes des ouvriers polonais à l'égard de la condition sociale de la femme et de l'organisation de la vie familiale.

Nous nous garderons de traiter nos deux échantillons, restreints comme ils sont et choisis d'une manière assez arbitraire, comme s'il s'agissait d'un échantillon parfait de l'ensemble de la population ouvrière polonaise. Cependant, ces groupes homogènes semblent représentatifs, au moins pour les ouvriers mariés, employés dans deux industries de grande importance et habitant de grandes villes. Là où les opinions diffèrent considérablement, les différences peuvent être attribuées, en général, à des facteurs connus de la situation économique et sociale des intéressés. Là où les opinions présentent un niveau élevé de concordance, elles peuvent être considérées comme caractéristiques d'une catégorie assez étendue d'ouvriers polonais.

Une étude comparative ayant pour objet les attitudes familiales et les opinions de l'homme en ce qui concerne la condition sociale de la femme touche à un problème quasi universel et peu révéler plusieurs traits caractéristiques d'une culture, à condition qu'elle tienne compte de ce que représente chaque groupe national considéré dans le cadre général de référence.

Les deux échantillons d'ouvriers polonais représentent la population d'un pays de l'Europe de l'Est qui, par sa culture littéraire et la façon de vivre de ses classes supérieures, était fortement attaché à la tradition occidentale. Il s'agit d'un groupe ouvrier d'un pays où l'industrie s'est développée pendant plus de cent ans au sein d'une économie agricole et de la structure sociale correspondante ; d'un pays, enfin, dont la 
transformation économique et sociale dans le sens de l'industrialisation moderne a été réalisée dans le cadre du socialisme.

\section{b) Les conditions de vie et la gestion du budget}

$\underline{\text { Retour à la table des matières du } 1^{\mathrm{er}} \text { article sur les Polonaises }}$

Les conditions de vie actuelles des familles ouvrières en Pologne et les traditions de la vie polonaise, la politique économique et sociale des autorités et l'influence de l'idéologie socialiste, l'empreinte laissée dans les esprits par les divers moyens d'information, tels sont les facteurs principaux qui motivent les attitudes des sujets interrogés. Notre recherche n'a pas pu pénétrer au fond du mécanisme qui règle le comportement réel; elle se concentre sur les normes ou les modèles des rôles et des relations familiales acceptés dans le milieu ouvrier de deux villes ${ }^{1}$.

Le problème du travail féminin est d'une grande importance pour la recherche portant sur les attitudes des maris à l'égard de la condition de la femme dans la famille et dans la société. L'article consacré exclusivement à ce problème montre que les opinions des ouvriers polonais sont, en principe, très favorables au travail salarié des femmes, mais qu'il existe une ambivalence des attitudes, qui apparaît quand ce problème est traité du point de vue de la situation familiale du sujet interrogé, et non pas dans des termes généraux.

Les opinions exprimées en ce qui concerne les autres aspects du rôle de la femme ne sont pas sans ambiguïté non plus. Nous avons choisi, à titre d'exemples, certains de ces aspects, parmi lesquels le problème de la gestion du budget familial et, plus généralement, des décisions à prendre dans la vie familiale.

Comme M. Chombart de Lauwe l'a démontré pour les familles ouvrières françaises, l'obligation de gérer le budget, qui est imposée à la femme, représente plutôt une lourde charge qu'une preuve de supériorité reconnue. La même conclusion semble justifiée pour les familles ouvrières polonaises qui font l'objet de notre étude. Nous avons trouvé que la proportion des maris qui veulent charger exclusivement leur femme des responsabilités budgétaires est inverse de la proportion des femmes travaillant hors de chez elles. À Varsovie, 59\% des hommes déclarent que la femme doit gérer seule l'argent de la famille, contre $30 \%$ de réponses comparables à Lodz. Les maris de Lodz, beaucoup plus souvent que ceux de Varsovie, déclarent que cette obligation devrait être partagée entre l'homme et la femme (Lodz, 66\% Varsovie, $24 \%)$.

Il n'y a pas de différences aussi marquées dans les réponses concernant les autres décisions à prendre dans la vie familiale. La tendance à imposer les mêmes obligations à l'homme et à la femme est presque aussi forte dans les deux échantillons.

$1 \quad$ Il ne faut pas en conclure que les opinions des sujets interrogés soient toujours plus évoluées que les modes de leur comportement réel. Par exemple, l'aide des maris pour les travaux ménagers, selon le témoignage des épouses, est beaucoup plus étendue que ne l'auraient laissé entendre les déclarations des intéressés concernant les devoirs de l'homme à la maison. 
Voici l'opinion des maris de Lodz sur ce que devrait être la participation de l'homme et de la femme à diverses décisions intéressant le ménage.

$\begin{array}{lcccc} & \begin{array}{c}\text { L'homme doit } \\ \text { décider }\end{array} & \begin{array}{c}\text { La femme doit } \\ \text { décider }\end{array} & \begin{array}{c}\text { Le couple doit } \\ \text { décider }\end{array} & \text { Pas d'opinion } \\ \text { Grosses dépenses } & \% & \% & \% & 88 \\ \text { Éducation des enfants } & 4 & 8 & 89 & - \\ \text { Vacances et congés } & 2 & 8 & 89 & 3\end{array}$

Les attitudes des maris à l'égard de la condition de la femme dans le ménage sont reflétées aussi par leurs opinions quant au niveau d'instruction et au niveau de salaire qu'ils jugent équitables pour la femme par rapport à l'homme. Ici encore les attitudes des deux échantillons sont assez rapprochées, celles des maris de Lodz étant plus égalitaires. Les Varsoviens attachent plus d'importance au facteur éducation et la moitié d'entre eux souhaitent le même niveau d'instruction pour la femme et le mari, $3 \%$ seulement étant en faveur d'une instruction plus poussée pour la femme et $18 \%$ en faveur d'une instruction moins poussée. À Lodz, plus d'un tiers des maris souhaitent une instruction égale, $10 \%$ une instruction plus poussée et $12 \%$ une instruction moins poussée pour la femme; pour le reste des sujets interrogés, le problème ne présente pas d'importance.

\section{c) L'égalité de l'homme et de la femme et les modèles familiaux}

$\underline{\text { Retour à la table des matières du } 1^{\mathrm{er}} \text { article sur les Polonaises }}$

Les maris semblent plus sensibles à la rivalité des deux sexes quand les facteurs de salaire et de hiérarchie professionnelle entrent en jeu : $29 \%$ des maris dans le groupe de Lodz et $15 \%$ dans celui de Varsovie considèrent qu'il est préférable que la femme gagne autant que son mari ; $25 \%$ à Lodz et $41 \%$ à Varsovie considèrent qu'il est préférable que la femme gagne moins ; $15 \%$ à Lodz et $4 \%$ seulement à Varsovie acceptent qu'elle gagne davantage. La condition de la femme, sur le plan professionnel, devrait être égale à celle du mari pour 25\% des hommes de Lodz et pour $14 \%$ de ceux de Varsovie ; elle devrait être inférieure pour $28 \%$ des sujets interrogés à Lodz et pour $30 \%$ des sujets interrogés à Varsovie. Le pourcentage de ceux qui n'attachent pas d'importance à la situation relative de l'homme et de la femme varie, selon la question et le groupe, de $25 \%$ à $50 \%$.

Il est évident qu'une proportion assez élevée de maris ne veulent pas accepter l'égalité des femmes sur le plan professionnel. Cette répugnance est sans doute liée à l'image de la femme au foyer. Le modèle de la femme, ainsi que celui du mari, est une notion générale globale, partiellement indépendante des opinions individuelles sur les attributs particuliers de chacun des conjoints (rôle et condition). 
Ces modèles globaux étaient présentés dans les réponses des intéressés à la question « Comment doit être, selon vous, un bon Père de famille ? » et à la question analogue concernant le modèle de la femme en tant qu'épouse.

Les réponses concernant le modèle du père de famille ont été classées en huit catégories, qui mettaient en évidence les traits de personnalité aussi bien que le mode de comportement. La plus grande partie des réponses entraient dans quatre catégories caractérisant le père de la façon suivante

Il doit gagner un salaire suffisant pour assurer la vie de la famille : Lodz, 64,6\% des sujets interrogés ; Varsovie, 58\%.

Il doit donner un bon exemple à la famille, ne pas boire : $44,5 \%$ et $45 \%$

Il doit être attaché à sa maison : $33,3 \%$ et 40

Il doit s'intéresser à l'éducation des enfants : $23,2 \%$ et 28

Pour le modèle de la femme, la concentration des réponses s'est opérée dans trois catégories :

Bonne ménagère : $75,8 \%$ et 87

Ayant bon caractère : $49,5 \%$ et 39

Bonne mère : $33,7 \%$ et $44 \%$

On pourrait dire que, dans le modèle du père comme dans celui de la mère, les qualités pratiques sont les plus notées. Les demandes d'affection et d'amour n'apparaissent que chez une minorité des sujets interrogés.

Les résultats de l'enquête n'autorisent pas à tirer de conclusions en ce qui concerne le comportement des sujets interrogés, non plus que les modèles réels de leurs relations sociales. On peut en conclure seulement que les normes acceptées dans le milieu ne favorisent pas l'expression des sentiments. Le modèle de la famille est très éloigné du modèle romantique et il souligne surtout l'aspect pratique du mariage et de la vie familiale. Dans le modèle du mari, c'est le rôle du «soutien de famille » (provider) qui prend la place prépondérante ; dans le modèle de la femme, c'est le rôle de la bonne ménagère.

J'ai présenté ailleurs un modèle de la famille rendu populaire, entre 1949 et 1955 , par un magazine féminin polonais à grand tirage. Selon ce modèle, le travail professionnel était bien intégré à la vie familiale et apportait une solution à tous les problèmes personnels de la femme, et non pas seulement aux difficultés matérielles de la famille. Or l'idée que se font les sujets interrogés de la place du travail dans la vie familiale diffère considérablement de ce modèle. Si les hommes reconnaissent aux femmes le droit d'occuper des emplois dans la production, le commerce et dans d'autres branches d'activité professionnelle, s'ils se déclarent même persuadés de la supériorité des ouvrières dans certains métiers, et partisans de l'égalité des salaires, à l'intérieur de la famille ils sont enclins à soutenir la distinction traditionnelle du rôle féminin et du rôle masculin. La majorité des sujets émettent l'opinion que seul l'homme doit gagner la vie de la famille.

En même temps, le modèle familial semble assez éloigné de l'image traditionnelle de la famille patriarcale. Les sujets interrogés n'insistent pas sur la position dominante 
du mari en tant que chef de famille ; 2 seulement à Lodz et 6 à Varsovie emploient de telles expressions en parlant du père. Il faut rappeler ici les opinions sur la question du budget et la participation commune de l'homme et de la femme aux décisions importantes à prendre. Selon les déclarations de la majorité des sujets, la femme doit être aidée par le mari dans les travaux du ménage et dans les soins à donner aux jeunes enfants. À Lodz, tous les hommes sans exception demandent aux enfants le même respect et la même obéissance pour le père et pour la mère; à Varsovie, le nombre des partisans de la prépondérance de l'autorité paternelle n'est pas grand : 7 personnes en tout.

Nos échantillons restreints ne nous permettent pas d'établir une corrélation positive ou négative entre, d'une part, les déclarations égalitaires et, d'autre part, l'âge des sujets et le fait que leurs femmes travaillent actuellement au dehors ou non. Il faut noter, cependant, que les ouvriers de Lodz se montrent, en général, plus égalitaristes dans leurs opinions que ceux de Varsovie. Or les ouvriers de Lodz ont un niveau d'instruction plus bas, ils sont moins qualifiés professionnellement et ils viennent de la campagne dans une plus large proportion que ceux de Varsovie. Leurs attitudes à l'égard de la condition sociale de la femme sont cependant plus évoluées, ou, si l'on veut, plus progressistes.

Une hypothèse semble particulièrement juste pour expliquer cette différence d'opinion : quelle que soit l'expérience personnelle des maris de Lodz en ce qui concerne le travail féminin, ils sont influencés par le milieu, où, traditionnellement, la femme contribue à gagner la vie de la famille et où l'on peut difficilement se passer de son concours ${ }^{1}$. Cette situation n'a pas effacé de l'image du rôle masculin son attribut de «seul soutien de la famille », mais elle a contribué à rehausser la place de la femme dans le modèle familial.

Ce trait met en relief le rôle que le travail féminin peut jouer dans l'établissement de l'égalité des deux sexes, sous certaines conditions. Mais ce n'est pas, certainement, la seule influence qui modifie les attitudes. La propagande officielle en faveur de l'émancipation de la femme peut aussi jouer un rôle, d'autant plus qu'il s'agit ici de déclarations des sujets interrogés et non pas de l'observation de leur comportement réel.

Au cours de l'analyse précédente du modèle de la famille qui était axée sur la place de la femme dans la structure familiale, deux tendances différentes se sont esquissées : l'une visant à Préserver la distinction traditionnelle des rôles féminin et masculin, l'autre visant non seulement à reconnaître aux femmes une condition égale à celle de leur mari, mais à minimiser la différence des rôles respectifs des deux sexes, ou, du moins, la valeur hiérarchique de cette différence. Ce qui est le plus caractéristique dans les attitudes des sujets interrogés, c'est que les deux tendances se manifestent souvent dans les opinions des mêmes personnes. Leur actualisation alternative est visiblement liée aux situations différentes envisagées par les intéressés au cours de l'interview.

V. A. Kloskowska, op.cit. Selon une étude faite en 1927 et portant sur 113 familles ouvrières de Varsovie et 72 familles de Lodz, le salaire du chef de famille représentait 86,3\% des revenus de la famille à Varsovie et $71,1 \%$ à Lodz. 


\section{d) L'avenir des enfants}

$\underline{\text { Retour à la table des matières du } 1^{\mathrm{er}} \text { articles sur la Pologne }}$

La tendance égalitaire devient particulièrement forte quand les sujets interrogés parlent non pas de leur femme, mais de leurs filles, quand ils considèrent les problèmes d'éducation et projettent les conceptions du rôle féminin sur l'avenir de leurs enfants.

La majorité des sujets se déclarent partisans de l'égalité des méthodes d'éducation morale des garçons et des filles, quoiqu'une proportion assez considérable, allant jusqu'à un tiers à peu près à Lodz, pense qu'il faut plus de soins et plus de surveillance pour les filles et des méthodes plus strictes avec les garçons.

En ce qui concerne l'instruction proprement dite, une pareille division des opinions ne se retrouve pas. Plus de $80 \%$ des sujets des deux échantillons - $86 \%$ à Lodz et $83 \%$ à Varsovie - préconisent une égalité absolue du niveau et du programme des cours pour les enfants des deux sexes. Ces déclarations coïncident avec la façon dont les intéressés se représentent l'avenir de leurs enfants.

On se souvient qu'en parlant du modèle de la femme, la plupart des sujets interrogés insistaient sur son rôle de bonne ménagère. Un seul membre de l'échantillon de Lodz et 7 de celui de Varsovie ont parlé du rôle futur de leurs filles dans les mêmes termes. Tous les autres ont souhaité pour leurs filles, comme pour leurs fils, un métier défini. Indiquant les professions envisagées pour leurs enfants, $54 \%$ des sujets de Lodz ont cité une profession exigeant des études supérieures, en parlant de leurs filles, et $59 \%$ en parlant de leurs fils; la différence n'est pas significative et, dans le groupe de Varsovie, la proportion est même inversée.

Il est vrai que le choix des professions n'est pas le même. La profession le plus souvent choisie, pour les filles, est celle de médecin (31\% des sujets interrogés, à Lodz comme à Varsovie) et, pour les garçons, celle d'ingénieur (31\% des sujets interrogés à Lodz et $26 \%$ à Varsovie). Les professions choisies pour les deux sexes ne sont donc pas identiques, mais elles occupent la même place dans l'échelle du prestige social et elles demandent le même niveau d'instruction.

Avant la guerre, les aspirations des familles ouvrières polonaises n'allaient pas si loin. Une enquête effectuée en 1927 avait montré que, sur 113 familles ouvrières de Varsovie, 6,2\% seulement et, sur 72 familles ouvrières de Lodz, 12,5\% exprimaient le désir de voir leurs enfants faire des études supérieures ${ }^{1}$. Ces aspirations ainsi exprimées concernaient, de façon caractéristique, presque exclusivement les garçons,

Les résultats sont indiqués ici pour l'ensemble des familles interrogées. Selon l'opinion des enquêteurs, les indications des parents concernant l'éducation «supérieure » à donner à leurs enfants étaient si vagues que le pourcentage de ceux qui pensaient réellement à une carrière universitaire pour leurs enfants était, en réalité, encore plus faible. [Source : Warunki zycia robotniczego w Warszawie, Lodzi i Zaglebiu Dabrowskim (Conditions de vie ouvrière à Varsovie, à Lodz, et dans la région des charbonnages de Dabrowa Gornicza). Warszawa, 1929.] 
et non pas les filles. Les déclarations des parents ouvriers traduisaient assez exactement les chances réelles qui s'offraient à leurs enfants de faire des études supérieures. En 1934, la jeunesse d'origine ouvrière représentait, en Pologne, 6,4\% des étudiants ; la proportion de l'élément féminin y était minime. Actuellement, malgré des changements importants, on ne peut pas, non plus, parler de l'égalité numérique des sexes dans les établissements d'enseignement supérieur, à l'exception des universités ${ }^{1}$. Cependant, l'évolution du modèle semble plus radicale que celle du pattern de comportement. À la campagne même, on peut entendre exprimer l'opinion qu'il faut donner une éducation plus poussée aux jeunes filles plutôt qu'aux garçons, ces derniers pouvant se débrouiller plus facilement dans la vie. ${ }^{2}$

L'intérêt accordé à l'éducation et au travail scolaire des enfants, dans nos deux échantillons, est un trait nouveau des attitudes de la masse ouvrière polonaise. Son apparition est liée à l'avènement social d'une nouvelle classe. Pour mesurer l'importance de ce phénomène, il faut comparer la situation des parents - dont $49 \%$ dans le groupe de Lodz, ne possèdent même pas le certificat d'études primaires - avec leurs aspirations en ce qui concerne leurs enfants. Nous sommes ici loin du scepticisme des ouvriers d'avant guerre en ce qui concerne les possibilités d'éducation de la jeunesse ouvrière, et plus loin encore de la famille traditionnelle paysanne, dont le père exigeait une soumission absolue de son fils, qui, après sa mort, devait suivre le même chemin dans les cadres, presque immuables, de la vie économique et sociale de son village. Un père ouvrier, sachant à peine lire et écrire, qui destine son enfant à des études universitaires, ne peut plus le traiter de la même façon, même si ses aspirations ne sont pas réalisées.

Les possibilités offertes à la jeune génération, non seulement par le système d'éducation, mais surtout par l'expansion industrielle, font éclater la structure traditionnelle de la famille paysanne. Elles modifient les attitudes et les relations dans la famille ouvrière. Mais les changements rapides constituent aussi une source de confusion et d'ambivalence. L'ouvrier polonais qui manifeste une attitude favorable à l'égard du travail de la femme mariée veut garder sa femme à la maison. Son modèle de la femme est axé autour du rôle de la bonne ménagère, mais il se représente volontiers sa fille comme un futur médecin. Il est peu conscient des changements survenus, au cours d'une génération, dans les méthodes d'éducation et dans les rapports entre parents et enfants et, S'il insiste sur l'obéissance des enfants, dans l'image modèle qu'il présente des rapports parents-enfants, il veut que ses fils et ses filles s'élèvent au-dessus de lui, d'une manière telle que ses propres parents ne l'avaient jamais envisagée pour lui-même. Sur le plan de ses attitudes, comme sur celui des conditions matérielles, sa vie abonde en contrastes.

La proportion d'étudiants d'origine ouvrière dans les établissements d'enseignement supérieur polonais, en 1957-1958, était de 30,1\% l'élément féminin représentait 32,7\% de cette catégorie d'étudiants. Le pourcentage de cet élément, pour l'ensemble des étudiants, était de 37,2\%.

2 Opinion recueillie au cours d'une enquête menée dans un village d'une région montagneuse peu développée, par Z. Wierzbicki [«Oswiata i motywi ksztalcenia dzieci we wsi Zmiaca» (L'éducation et les motifs d'éduquer les enfants chez les paysans de Zmiaca), Przeglad socjologiczny, 1958]. Malgré ces déclarations, qui marquent un important changement d'opinion, le pourcentage des étudiantes est toujours faible dans le groupe des étudiants d'origine paysanne $(28 \%)$. 


\section{Considérations théoriques concernant les recherches comparatives sur les modèles sociaux}

$\underline{\text { Retour à la table des matières du } 1^{\mathrm{er}} \text { article sur les Polonaises }}$

Comme nous l'avons déjà souligné, les recherches entreprises, à Lodz et à Varsovie, sur les attitudes des ouvriers, ont permis de caractériser les opinions et les idées sur la condition de la femme dans la famille et dans la société (plutôt que de présenter les patterns de son comportement véritable dans la vie familiale et professionnelle). Dans les recherches sociologiques qui procèdent surtout par enquêtes, se produit quelquefois cette confusion entre deux structures sociologiques distinctes, que l'on pourrait désigner par les mots «modèles » et patterns. Nous employons ici le terme de pattern pour définir un comportement socio-culturel authentique; par contre, le terme de «modèle » désigne les normes et les idées des membres des échantillons interrogés au sujet de ces comportements.

Un pattern peut être plus ou moins conscient, mais il est toujours la représentation de comportements typiques véritables à l'intérieur d'une division sociale donnée. Un modèle, dans le sens que nous avons adopté ici, est toujours conscient ; qu'il soit formulé en paroles ou exprimé dans l'ensemble des normes ou des idées se rapportant à une sphère déterminée d'action sociale, il sert toujours à «faire connaître » des comportements. Sa fonction ne consiste d'ailleurs pas à rendre compte de la réalité, mais, en accord avec la définition de Levi-Strauss ${ }^{1}$, à propager et à étendre des types de comportements déterminés. Les modèles remplissent donc cette fonction et servent de mesure standardisée pour les comportements individuels, mais ils possèdent aussi une composante morale très nette.

Les modèles se reconnaissent à leur caractère normatif, même lorsqu'ils ne sont pas formulés en langage normatif, à proprement parler : ils expriment nettement de telles intentions à l'aide de jugements de valeur.

La littérature didactique pour enfants a toujours abondé en pareils modèles - qu'il s'agisse des «petites filles modèles » de la comtesse de Ségur ou du «petit garçon bien sage » des histoires lues dans les écoles du dimanche anglo-saxonnes. La littérature populaire de diverses époques abonde aussi en modèles de la vie de famille.

C. Levi-Strauss. Anthropologie structurale, la notion de structure en ethnologie. Paris 1958. La distinction entre modèles, dans le sens adopté ici, et patterns semble indispensable, indépendamment de la distinction des patterns avec prise de conscience et sans prise de conscience(overt et covert) de R. K. Merton, puisque ces overt patterns n'ont pas toujours un caractère normatif. 
Les modèles jouent un rôle important dans la pédagogie et dans la propagande, mais leur intervention ne se borne pas à ces deux domaines de la culture. En considérant les modèles du point de vue de leurs créateurs ou de leurs propagateurs, on peut en distinguer qui sont créés pour être utilisés par autrui. On peut ranger dans cette catégorie ceux qui sont répandus par la littérature, la presse et les autres moyens de grande information; ils sont formulés par des personnes ou des groupes remplissant des fonctions d'éducation ou de propagande, par les professeurs, les prédicateurs, les moralistes, les politiciens, les planificateurs sociaux.

À côté de ces modèles, servant à la propagande et produits par des organismes d'action sociale spécialisés, il en existe d'autres, dépendant du milieu, qui sont justement le produit des groupes au comportement desquels ils se rapportent directement. Ces deux catégories de modèles exercent, en général, une action réciproque l'une sur l'autre, mais la relation entre leurs contenus peut varier de l'identique au contraire. De même, la relation entre les modèles et les patterns de comportement n'a pas un caractère stable. Dans certaines cultures et à certaines époques de l'histoire, dans des conditions de grande stabilité et à l'intérieur de petits groupes homogènes, les modèles peuvent se rapprocher beaucoup des patterns de comportement. Par contre, au sein de groupes étendus et hétérogènes, subissant des transformations rapides, il semble qu'il, faille s'attendre à un décalage important entre les modèles et les patterns. Dans certains cas, les modèles, surtout ceux qui rentrent dans la catégorie "propagande », peuvent avoir un caractère de modèles «purs », sans aucune interaction avec la sphère de comportement social et, par conséquent, avec le domaine des patterns.

L'interview et l'enquête servent surtout à l'étude des modèles, alors que l'observation directe semble avoir une importance fondamentale pour l'étude des patterns de comportement. L'analyse des rapports entre les patterns et les modèles de la société étudiée constitue le véritable travail du chercheur. Cela ne signifie pas, cependant, que les patterns ou les modèles n'offrent pas, indépendamment les uns des autres, des sujets de recherche justifiés. Un travail très attrayant, par exemple, semble être offert par le classement des modèles intervenant dans les divers milieux d'une même société et se rapportant aux mêmes institutions, ou régissant le même genre de rapports sociaux. Un autre domaine non moins intéressant est celui de la comparaison des modèles de cultures différentes.

Les deux études présentées ici, qui portent sur la famille ouvrière polonaise, s'intéressent surtout aux idées et aux opinions de deux groupes d'ouvriers polonais sur la famille et sur la place de la femme dans la société. Elles présentent, en fait, un modèle du milieu familial assez homogène, malgré certaines différences dues à la spécialité professionnelle des deux échantillons.

La confrontation de ces modèles de groupe avec les modèles de propagande semble intéressante. Pour la comparaison, nous nous servirons ici de modèles présentés dans un journal féminin très populaire, qui compte beaucoup de lecteurs dans les deux groupes étudiés ${ }^{1}$. Nous donnerons ailleurs un résumé de l'analyse du modèle de la famille, faite sur la partie littéraire et sur la partie «Courrier du cœur» de ce journal, à deux époques différentes : 1950-1951 et 1956-1957. Nous appellerons ces modèles modèle de propagande no 1 et modèle de propagande no 2.

1 A. Kloskowska. « Changing family models in the popular magazines in Poland», op. cit. 
Le modèle de la famille défini comme modèle de propagande no 1 se caractérisait, avant tout, par une subordination stricte de la famille à un groupe plus large. Il ne représentait pas la conception traditionnelle et institutionnelle de la famille comme la voyait Burgess. Ce qui le distinguait surtout de cet autre type de conception, c'était la réduction du rôle de la famille. Cette réduction intervenait ici à l'échelle macroscopique comme à l'échelle microscopique. Le rôle de la famille se ramenait surtout à la procréation. Le modèle se distinguait par l'égalité des rapports à l'intérieur de la famille, liée au principe de non-accentuation des différences entre les rôles de la femme et ceux de l'homme. Ce principe découlait, à son tour, du fait que, l'accent était mis sur les fonctions extrafamiliales, professionnelles, du mari et de la femme, comme des parents et des enfants. La principale innovation de ce modèle était l'harmonie entre les fonctions familiales et productives, surtout dans le cas de la femme.

Le modèle de propagande no 2 se distinguait assez nettement du premier. Il se rapprochait beaucoup de la représentation nucléaire de la famille, comme la conçoit Parsons. Ici l'accent était mis principalement sur les fonctions expressives et intégratives et, par conséquent, sur le rôle de la femme en tant que personne et en tant que mère, sur le rôle de la famille dans la socialisation et dans l'expression de l'individu, Ce modèle reposait sur le principe d'individualisation des relations interpersonnelles. Les sentiments des époux et ceux des parents et des enfants y occupaient la place principale du point de vue de la valeur.

Le modèle de groupe se distingue du modèle de propagande no 1 par beaucoup de points importants. Une forte majorité des sujets interviewés Sont hostiles à la conception d'une famille dans laquelle la fonction «familiale » serait réduite et remplacée, en partie, par des institutions sociales comme les crèches, les écoles maternelles, les clubs, les cantines.

Le modèle de groupe instaure, en particulier, la différence entre les rôles fonctionnels de l'homme et de la femme au sein de la famille. Le travail en vue de faire vivre la famille constitue, selon ce modèle, le rôle de l'homme exclusivement. La conviction de l'incompatibilité des fonctions professionnelles et familiales de la femme ayant des enfants en bas âge y intervient fortement.

Le modèle de groupe se différencie aussi, par beaucoup de côtés, du modèle de propagande no 2. Dans la définition des rôles du mari et de la femme, il valorise les fonctions instrumentales des deux époux par rapport aux fonctions expressives. Il met donc l'accent sur le rôle du mari en tant que pourvoyeur de la famille et sur le rôle de la femme en tant que maîtresse de maison. Le caractère émotionnel des liens familiaux joue donc très peu ici. Dans les rapports entre époux, comme dans les rapports parents-enfants, on ne parle pas des sentiments pas le premier rang du point de vue de la valeur.

L'élément qui rapproche le modèle de groupe des deux modèles de propagande est celui qui établit un rapport d'égalité entre les époux : le fait que, dans beaucoup de domaines de la vie familiale, la décision appartienne aux deux époux, et qu'il incombe au mari d'aider aux travaux de la maison et de participer aux soins donnés aux enfants les plus jeunes ; ces conceptions éloignent le modèle analysé du type traditionnel de famille patriarcale. Les « modèles » évoqués ici, puisés dans un journal populaire, ne constituent évidemment pas la seule source d'influence par propagande sur les modèles de groupe dans les échantillons étudiés. 
La ressemblance la plus importante entre notre modèle de groupe et les modèles de propagande caractéristiques des structures sociales actuelles en Pologne est sensible lorsque les sujets interrogés considèrent la condition sociale de la femme non pas dans la perspective de ses problèmes personnels, mais à l'échelle plus large des rapports sociaux.

Un phénomène analogue d'ambivalence des attitudes a été observé par V. Talmon-Garber ${ }^{1}$, dans ses recherches sur les patterns et les modèles de la famille dans les kibboutzim d'Israël. Là aussi, la distance entre les modèles de groupe et les modèles de propagande, de caractère idéologique, se transformait selon que les normes et les appréciations formulées par les sujets interrogés se rapportaient à des problèmes de la vie courante ou à des phénomènes sociaux d'ordre plus général.

C'est justement dans les déclarations de caractère général sur l'exercice d'une profession et sur la place sociale de la femme que l'attitude des échantillons étudiés dans le milieu ouvrier polonais diffère le plus sensiblement de celle qui est exprimée par l'échantillon d'ouvriers français interviewés ${ }^{2}$. L'acceptation très répandue du travail de la femme - y compris la femme mariée - formulée comme un principe général dans l'échantillon de Lodz et de Varsovie, fait contraste avec l'attitude des ouvriers français.

Dans l'étude des opinions des deux échantillons polonais, on ne trouve pas du tout d'arguments contre le travail des femmes, comme ceux qui sont avancés par les ouvriers français : par exemple, la crainte de la concurrence et la conviction de l'influence négative du travail en usine sur la moralité des femmes. Les préjugés contre le travail de la femme liés à la peur d'une perte de prestige au sein de la famille, fréquents chez les ouvriers français, sont très rares parmi les ouvriers polonais.

Dans l'état actuel des recherches, il est difficile d'obtenir une analyse plus précise comparant les données françaises et polonaises. Les attitudes à l'égard du statut de la femme dans la famille et dans la société s'appuient, dans chaque échantillon, sur des conditions économiques et sociales différentes. Certaines particularités n'empêchent pas, actuellement, le développement de l'emploi des femmes en Pologne. Les influences des idéologies traditionnelles de la famille, dans les deux pays, diffèrent aussi sous certains aspects.

La rapidité de l'industrialisation et de l'urbanisation en Pologne influe sur l'inégalité de la transformation des patterns et des modèles et trouve son expression dans l'ambivalence des attitudes, qui a été soulignée plus d'une fois dans l'analyse des données polonaises. Une telle ambivalence, constituant l'expression des processus d'accroissement et de développement était certainement moins sensible dans les attitudes des ouvriers français.

V.Talmon-Garber. « Social structure and family size », in Human relations, vol. XII, no 2, 1959.

Les attitudes des mineurs du bassin houiller de Slansk (présentées dans l'articlede M. Piotrowski) diffèrent beaucoup de celles des échantillons de Lodz et de Varsovie. Les attitudes de ce groupe se rapprochent davantage de celles de certains échantillons français. Les mineurs formant un groupe professionnel très spécifique et un groupe religieux très spécifique aussi, leurs attitudes ne peuvent être rapprochées de celles des ouvriers des industries du centre de la Pologne. 
II

Les attitudes à l'égard de la condition de la femme

dans les familles ouvrières polonaises

\section{II}

\section{"Attitudes à l'égard du travail des femmes"}

\section{J. Piotrowski [Université de Varsovie]}

Le problème du travail des femmes, notamment des femmes mariées, et de l'attitude adoptée à cet égard, par les femmes elles-mêmes ainsi que par leurs maris, a fait l'un des principaux objets des études effectuées en Pologne sur les familles ouvrières ${ }^{1}$.

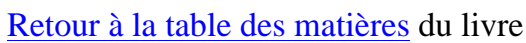

Table des matières du $2^{\mathrm{e}}$ article sur les femmes en Pologne

1. L'emploi des femmes en Pologne

2. Étude des attitudes enregistrées à l'égard du travail des femmes dans les familles ouvrières polonaises

3. L'attitude des maris a l'égard du travail de leur épouse

4. L'attitude des femmes mariées envers le travail

5. Quelques conclusions

1 Voir l'article de A. Kloskowska. 


\section{L'emploi des femmes en Pologne}

$\underline{\text { Retour à la table des matières du } 2^{\mathrm{r}} \text { article sur les Polonaises }}$

En Pologne, le travail des femmes suscite un vif intérêt théorique et pratique en tant que problème sociologique et économique d'actualité.

Les femmes représentent un tiers de l'effectif total des ouvriers et employés. Dès avant la guerre, le pourcentage des femmes était le même par rapport au chiffre total des travailleurs. Cependant, la Pologne d'avant guerre était un pays où les petites entreprises agricoles, ainsi que le petit commerce et l'artisanat, constituaient la partie prépondérante de l'économie, tandis que les travailleurs formaient un groupe relativement peu nombreux au sein de la population. Les femmes qui occupaient un emploi ne constituaient donc qu'une catégorie assez restreinte parmi les femmes en âge de travailler. Après la guerre, à la suite de l'industrialisation et des transformations sociales et économiques qui se sont opérées en Pologne, ces rapports se sont considérablement modifiés. La population active (travailleurs et leurs familles), qui ne représentait, avant la guerre, que $17 \%$ environ du total des habitants, en représente aujourd'hui plus de 55\%, Avant la guerre, sur dix femmes âgées de 16 à 64 ans, une à peine occupait un emploi. De nos jours, une sur quatre a un emploi. Dans les régions urbaines et industrielles, le pourcentage des femmes travaillant hors de leur domicile est naturellement beaucoup plus élevé. Il convient, en outre, de souligner qu'avant la guerre près de $40 \%$ des femmes qui travaillaient étaient employées en tant que domestiques. Aujourd'hui, ce type d'emploi devient de plus en plus rare. C'est là une donnée intéressante, lorsqu'il est question du travail des femmes mariées. En effet, le métier de domestique offrait généralement peu de possibilités aux femmes mariées.

Les femmes ayant acquis des droits égaux à ceux des hommes, y compris le droit à l'instruction et au travail, on peut observer que beaucoup dés femmes qui travaillent possèdent des qualifications professionnelles. D'après les données de 1959, sur 1000 travailleurs, 31 possédaient un diplôme universitaire et 44 un certificat d'études Professionnelles secondaires, alors que, sur 1000 femmes qui travaillaient, 25 possédaient un diplôme d'études universitaires et 94 un certificat d'études Professionnelles. Les jeunes filles constituent $35 \%$ du contingent des élèves sortant des écoles professionnelles secondaires et 30\% des diplômés des universités. La préférence des femmes pour certains métiers est visiblement marquée. Les femmes représentent $28 \%$ du total des employés qui ont fait des études universitaires, mais le pourcentage varie considérablement suivant les professions : 10\% pour les ingénieurs, mais $45 \%$ des diplômés ès sciences et $54 \%$ des diplômés ès lettres. Il y a $19 \%$ de femmes parmi les juristes, $30 \%$ parmi les économistes, $36 \%$ parmi les médecins et le nombre des femmes qui exercent leur activité dans ces trois domaines augmente toujours et est le plus élevé pour la génération la plus récente. Les femmes titulaires d'un certificat 
d'études professionnelles sont surtout nombreuses dans le secteur des activités économiques (66\% du total des employées), dans l'enseignement $(71 \%)$ et dans les services de santé $(96 \%)$.

La demande accrue de main-d'œuvre féminine, qui est le résultat direct du développement économique du pays ${ }^{1}$, offre aux femmes des possibilités toujours plus larges de trouver et de pratiquer le travail qui leur convient le mieux. Pour cette raison, comme pour d'autres, y compris les changements survenus dans les opinions, les mœurs et les idées sous l'influence de l'idéologie socialiste, les femmes mariées sont toujours plus nombreuses à exercer une activité salariée. Le nombre des femmes mariées, travaillant hors de leur domicile a augmenté, durant les quinze années révolues, plus rapidement que celui des femmes non mariées. D'après le recensement de 1931, le nombre des femmes mariées qui travaillaient s'élevait à environ 160000 et représentait environ $16 \%$ du total des femmes qui travaillaient. Aujourd'hui, ce nombre est d'environ 750000 (soit 30\% du total des femmes qui travaillent). Alors que le nombre des femmes non mariées qui travaillent a presque doublé par rapport à l'avant-guerre, le nombre des femmes mariées qui travaillent a presque quintuplé ${ }^{2}$. Pour la population non agricole, la proportion des femmes mariées travaillant hors de leur domicile est estimée à 25 ou $30 \%$. ${ }^{3}$ Presque toutes ces femmes sont employées à plein temps, tout comme leurs compagnes qui ne sont pas mariées. L'emploi des femmes mariées à temps partiel est un fait rare et toujours exceptionnel, quoiqu'un certain progrès se fasse déjà remarquer dans ce domaine.

Le travail des femmes mariées est favorisé, en premier lieu, par de plus grandes possibilités d'emploi, par l'accès à des emplois convenant mieux aux femmes et, enfin, par divers privilèges, découlant, entre autres, du développement d'une législation du travail qui tient largement compte des besoins et des intérêts de la mère qui travaille ${ }^{4}$.

Il semble que l'emploi des femmes devienne un modèle social ${ }^{5}$ (social pattern) de plus en plus généralement reconnu. Au sein de la population non agricole, les femmes célibataires, capables de travailler, ont presque toutes un emploi. Dans les villes importantes, le pourcentage des femmes mariées qui travaillent atteint près de $50 \%$, et, dans la majorité des cas, les intéressées sont employées de façon continue. Il semble qu'un travail rémunéré devienne, de plus en plus, un élément essentiel du rôle

De 1947 à 1959, le nombre des emplois a triplé, passant de 2500000 à 7500000.

Les chiffres exacts ne seront connus qu'après l'étude des résultats du recensement effectué en Pologne en décembre 1960. L'auteur a donc dû s'en tenir à ses propres évaluations, basées sur les données statistiques relatives au versement es allocations familiales. Les résultats de ces évaluations ainsi que la méthode employée ont été présentés dans un article paru dans la Revue des assurances et problèmes sociaux (actuellement : Travail et sécurité sociale) [Przeglad Ubezpiezen Spolecnycg i Zagadnien Socjalnych, n 3, 1959.]

3 À ce résultat a dû contribuer, entre autres, le fait que le pourcentage des femmes mariées s'est sensiblement accru en Pologne depuis la guerre, notamment dans la population non agricole. On voit maintenant les femmes se marier en plus grand nombre et à un plus jeune âge qu'avant la guerre.

4 D'après les sondages effectués par l'auteur, la plupart des femmes mariées qui travaillent ont des enfants en bas âge. Près de la moitié d'entre elles ont des enfants d'âge pré-scolaire.

5 La preuve en est donnée, entre autres, par la continuité croissante de l'emploi, par la moyenne plus élevée de l'âge des femmes qui travaillent et par le niveau plus élevé de leur formation et de leur qualification professionnelles. 
social de la femme mariée dans les milieux travailleurs, de son rôle dans la famille et dans la nations. ${ }^{1}$

\section{2. Étude des attitudes enregistrées à l'égard du travail des femmes dans les familles ouvrières polonaises}

$\underline{\text { Retour à la table des matières du } 2^{\mathrm{r}} \text { article sur les Polonaises }}$

Comment ces problèmes se reflètent-ils sur les opinions et les attitudes des familles ouvrières en Pologne?

Des recherches à ce sujet ont été effectuées essentiellement dans deux communautés : celle de Varsovie et celle de Lodz. Cependant, ces problèmes ont également fait partie d'un vaste programme de recherches sur la famille ouvrière (mineurs), entreprises par l'Institut scientifique silésien de Katowice ${ }^{2}$. Nous disposons donc des résultats de recherches effectuées dans trois milieux ouvriers différents. À Varsovie, l'étude a porté sur 100 familles d'ouvriers métallurgistes, pour la plupart spécialisés et percevant de hauts salaires, qui vivaient dans le milieu très différencié de la métropole. A Lodz, centre important de l'industrie textile, l'étude a porté sur 103 familles d'ouvriers du textile ; dans ce milieu, contrairement au milieu des travailleurs de la métallurgie, le travail des femmes est un phénomène habituel, relevant d'une tradition très ancienne. En Silésie, l'étude a porté sur 172 familles de mineurs, vivant dans deux petits «corons » très homogènes et quelque peu isolés (Murcki et Czerwionka), ayant de vieilles traditions de culture professionnelle.

Parmi les trois milieux étudiés, c'est dans les familles de mineurs que les maris gagnent le plus. C'est parmi les ouvriers du textile, à Lodz, que les revenus des maris sont les moins élevés. Les possibilités d'emploi offertes aux femmes sont vastes et variées, à Varsovie et à Lodz. Dans les corons de Silésie, qui ont fait l'objet de l'étude, les femmes n'ont que des possibilités très restreintes de trouver un emploi dans la localité où elles habitent. Chacun de ces trois milieux ouvriers se caractérise par des rapports différents en ce qui concerne le travail féminin, surtout celui des femmes mariées. Les estimations approximatives dont l'auteur a déjà fait mention indiquaient qu'en Pologne, pour l'ensemble des familles de travailleurs du textile, la proportion des épouses ayant un emploi se montait à 67\%. Pour les familles de mineurs, cette proportion était d'environ $15 \%$ et, pour les métallurgistes, elle était d'environ $29 \%$ Dans les milieux échantillonnés pour l'étude, les chiffres étaient les suivants : $35 \%$

1 Le problème du travail et du ménage, considéré du point de vue du rôle social de la femme mariée, a été approfondi par l'auteur dans une étude qui sera publiée prochainement.

2 Ces recherches étaient effectuées sous la direction de Mme Wanda Mrozkowa, agrégée de 1'Institut silésien. 
des épouses travaillaient à Varsovie (familles de métallurgistes), 68\% à Lodz (ouvriers du textile), $7 \%$ à peine en Silésie (familles de mineurs).

Les attitudes et les opinions révélées par l'étude ont fait apparaître des différences assez marquées entre les milieux de Varsovie et de Lodz, d'une part, et celui de Silésie, de l'autre. Les divergences d'attitudes entre les travailleurs du textile de Lodz et les métallos de Varsovie sont apparues moins prononcées qu'on n'aurait pu le présumer en partant des traditions si différentes de ces deux milieux en ce qui concerne le travail féminin.

\section{L'attitude des maris à l'égard du travail de leur épouse}

$\underline{\text { Retour à la table des matières du } 2^{\mathrm{r}} \text { article sur les Polonaises }}$

Il convient de noter, en premier lieu, qu'aussi bien à Varsovie qu'à Lodz la grande majorité des ouvriers $(90 \%$ se sont prononcés pour une pleine égalité des droits de la femme dans le domaine de l'emploi, y compris le droit au salaire égal pour un travail égal. La plupart des ouvriers considèrent qu'il y a de nombreux métiers dont les femmes s'acquittent mieux que les hommes. Ils soulignent, en même temps, en citant des exemples, qu'il y a bon nombre de métiers qui ne conviennent pas aux femmes et peuvent même leur porter préjudice. Cela ne signifie pas qu'il y ait une distinction entre métiers considérés comme supérieurs ou comme inférieurs. Il s'agit surtout d'opposer les travaux pénibles, moins propres, plus épuisants, fatigants, nuisibles aux femmes, aux travaux plus faciles, plus propres, plus spécialisés et surtout à ceux qui «n'usent pas » les femmes prématurément. Il est curieux que de nombreux ouvriers (35\% à Varsovie et 48\%, à Lodz) aient estimé que les femmes sont capables d'occuper des postes de techniciens et de cadres dans l'industrie et qu'elles conviennent encore mieux à des postes dirigeants dans l'administration (c'est l'opinion de $70 \%$ des ouvriers de Varsovie et de $73 \%$ de ceux de Lodz).

À la question i : «Les femmes non mariées peuvent-elles et doivent-elles travailler ? »94\% des ouvriers varsoviens et $100 \%$ de ceux de Lodz ont donné une réponse positive. Les métallurgistes de Varsovie qui se sont prononcés contre le travail des femmes non mariées avaient d'ailleurs cru qu'il s'agissait de jeunes filles n'ayant pas encore atteint l'âge du mariage et avaient invoqué l'argument selon lequel les intéressées devraient plutôt songer à s'instruire.

Les mineurs de Silésie sont d'avis que les femmes mariées peuvent travailler, mais ils ne pensent pas qu'elles «doivent » le faire, estimant que le rôle véritable de la femme est celui d'épouse et de mère de famille. À leur avis, une fois mariées, les femmes renonceront à leur travail; elles n'ont donc guère besoin d'une formation professionnelle. Et, en effet, il est de règle que, dans les familles de mineurs étudiées, 
les femmes mariées n'aient généralement pas de qualifications professionnelles ; $30 \%$ seulement d'entre elles avaient travaillé avant leur mariage et encore n'étaient-elles employées que comme manœuvres dans les emplois les plus mal rémunérés. Les mineurs silésiens ont gardé la notion de la «jeune fille destinée au mariage », attendant tranquillement son « promis » et se préparant, dans le foyer familial, à son rôle futur de mère et de ménagère. Les femmes elles-mêmes pensent généralement que le mariage les oblige à se retirer dans leur foyer et à renoncer à tout travail hors de leur domicile. Songent-elles parfois à gagner de l'argent ? C'est généralement en tant que couturières travaillant à domicile. La jeune génération, les filles adolescentes des familles sur lesquelles a porté l'étude, font preuve d'attitudes un peu différentes : sur 56 filles non mariées, 19 seulement restent à la maison, en attendant de trouver un mari. Les autres travaillent ou poursuivent leur études. Cependant, le nombre de celles qui tâchent d'acquérir des titres professionnels est toujours relativement restreint.

À Varsovie, 75\% des ouvriers et à Lodz $89 \%$ sont d'avis que les femmes mariées peuvent et doivent même travailler, lorsqu'elles n'ont pas d'enfants. En Silésie, de telles opinions sont rares. Deux seulement parmi les maris interviewés pensent que les femmes mariées sans enfants devraient travailler. À Varsovie et à Lodz semble prévaloir l'opinion : «A quoi bon, pour une femme mariée, rester chez elle, quand elle n'a pas d'enfant ? » En Silésie, par contre, domine l'opinion selon laquelle «ce n'est pas la peine de se marier, si la femme ne doit pas demeurer au foyer, si elle ne s'occupe pas de son ménage ni de son mari ». Il convient de souligner également, à propos des opinions exprimées à Varsovie, comme à Lodz, que les maris sont un peu moins en faveur de l'idée du travail des femmes mariées sans enfant.

Quant au travail des mères de jeunes enfants, les opinions sont nettement négatives : $92 \%$ des ouvriers de Varsovie et $95 \%$ de ceux de Lodz sont opposés au travail de ces femmes. En Silésie, on s'oppose plus énergiquement encore au travail des femmes, en évoquant surtout des traditions, des mœurs et des coutumes (par exemple, l'argument du prestige du mari, père de famille). À Lodz et à Varsovie, on a pu constater des attitudes beaucoup plus rationalistes. Dans ces milieux, si l'on s'oppose au travail des femmes mariées, c'est à cause du préjudice que le travail de la mère peut porter aux enfants, au ménage. On craint que la fatigue n'enlaidisse la femme, ne lui fasse renoncer à soigner son apparence.

Les réponses aux questions concernant non pas le travail des femmes en général, mais celui de la propre femme du sujet interviewé, font apparaître les opinions sous une lumière un peu différente. Là, le principe du droit égal de la femme au travail est limité par les exigences qu'on oppose à la femme au nom de l'intérêt des enfants et du foyer, des rapports entre époux. Presque tous les ouvriers se prononcent pour l'égalité des salaires masculins et féminins. Cela n'empêche pas que $41 \%$ des maris à Varsovie et $25 \%$ à Lodz trouvent qu'une femme doit toujours gagner moins que son mari. Environ $30 \%$ de ces maris préféreraient que la femme occupe un poste subalterne par rapport à celui du mari. Dans le cas contraire, selon eux, les rapports entre époux risquent d'être toujours un peu tendus. La grande majorité des maris estiment également que c'est l'époux qui doit gagner la vie de toute la famille. Cette opinion est d'ailleurs partagée par de nombreuses épouses. 


\section{Attitudes enregistrées dans 100 familles d'ouvriers polonais à l'égard du travail salarié de l'épouse (chiffres relatifs).}

\begin{tabular}{|c|c|c|c|c|}
\hline \multicolumn{2}{|c|}{$\begin{array}{l}\text { Métallurgistes } \\
\text { de Varsovie }\end{array}$} & \multicolumn{3}{|c|}{$\begin{array}{l}\text { Ouvriers du textile } \\
\text { de Lodz }\end{array}$} \\
\hline 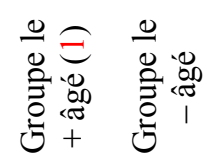 & 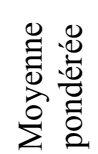 & 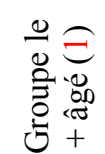 & 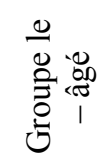 & 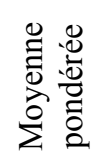 \\
\hline
\end{tabular}

Nombre de familles

Où le mari est content que sa femme travaille

Où le mari voudrait que sa femme continue à travailler (2)

70

20

Où la femme veut continuer à travailler

80

Familles où l'épouse travaille

Motifs et nombre de réponses des épouses qui travaillent en

faveur du travail salarié

«Pour avoir plus d'argent pour le ménage »

« Pour être indépendante et avoir de l'argent bien à vous »

" Parce que votre travail vous semble intéressant»

« Pour fréquenter plus de gens, avoir une vie moins

48

54

$37 \quad 32$

$32 \quad 33$

4

8

$5 \quad 10$

9

monotone »

$\begin{array}{cccccc}70 & 100 & 91 & 100 & 100 & 100 \\ 50 & 16 & 23 & - & 13 & 9 \\ 40 & 4 & 14 & - & 6 & 4 \\ 60 & 20 & 31 & - & 4 & 3\end{array}$

Nombre de familles

Familles où l'épouse ne travaille pas

Où le mari est mécontent que sa femme ne travaille pas

Où le mari voudrait que sa femme commence à travailler

Où la femme voudrait commencer à travailler (3)

$\begin{array}{cccccc}0 & 11 & 8 & 25 & 40 & 36 \\ 5 & 49 & 36 & 50 & 60 & 58 \\ 40 & 69 & 60 & 87 & 72 & 76\end{array}$

Motifs et nombre de réponses des épouses qui voudraient commencer à travailler

« Pour avoir plus d'argent pour le ménage »

$\begin{array}{llllll}55 & 90 & 90 & 100 & 100 & 100\end{array}$

« Pour être indépendante et avoir de l'argent bien à vous »

$87 \quad 15$

33

$\begin{array}{lll}25 & 28 & 28\end{array}$

«Parce que votre travail vous semble intéressant »

$12 \quad 15$

$87 \quad 39$

15

$\begin{array}{lll}25 & 17 & 20\end{array}$

«Pour fréquenter plus de gens, avoir une vie moins monotone »

1. Familles du groupe le plus âgé : celles où le mari a plus de 45 ans.

2. La question était ainsi formulée: «Si les revenus de votre mari s'accroissaient au point de suffire à l'entretien du ménage, continueriez-vous à travailler?»

3. La question était : « Voudriez-vous travailler à l'avenir ?» 
Comme il a déjà été dit, des attitudes nettement négatives vis-à-vis du travail des épouses se manifestent parmi les mineurs. Dans les familles de métallurgistes et d'ouvriers du textile, bon nombre de maris font preuve d'hésitation et d'incohérence dans leur opinion ${ }^{1}$, lorsqu'il s'agit du travail de leur épouse (voir le tableau, p. 85)

Comme on peut le voir par les réponses, les attitudes défavorables au travail des épouses dominent; cependant, les maris satisfaits de ce que leur femme travaille, de même que ceux qui verraient avec plaisir travailler la leur, constituent un pourcentage assez considérable. Ce qui est frappant, c'est la divergence entre les réponses relatives à la satisfaction du mari, du fait que sa femme travaille ou ne travaille pas, et les réponses faites à la question de savoir si le mari désire que sa femme travaille. Les maris contents de voir leur femme travailler sont relativement nombreux, mais peu d'entre eux voudraient que leur femme continue à travailler. En revanche, dans les familles où les femmes ne travaillent pas, on trouve des maris qui, bien que contents de voir leur femme rester au foyer, n'en voudraient pas moins qu'elle travaille.

On remarque donc une ambivalence visible d'opinions, découlant d'une certaine rationalisation des attitudes. N'étant plus fondées sur des coutumes, les opinions deviennent fréquemment indécises. Les arguments sont considérés et reconsidérés. La plupart des maris voient non seulement les effets négatifs mais aussi les effets positifs du travail de leur femme. On énumère, comme effets négatifs, l'abandon des soins du ménage, des enfants, du mari. Parmi les côtés positifs, on voit surtout les avantages d'ordre financier ${ }^{2}$.

Il est intéressant d'observer à ce propos que le pourcentage des maris désireux de voir travailler leur femme est beaucoup moins élevé dans les familles où la femme travaille que dans celles où elle ne travaille pas. À cet égard, la situation de Lodz ressemble à celle de Varsovie, quoique les maris qui voudraient voir leur femme accroître quelque peu le revenu familial soient un peu plus nombreux à Lodz. Les besoins non satisfaits s'expriment plus nettement dans les attitudes que les besoins satisfaits. (Ce qui ne veut pas dire que les premiers soient plus importants.) Lorsque la situation financière de la famille est meilleure, notamment parce que la femme travaille, le mari est plus sensible à « l'abandon du ménage » et veut y remédier en faisant renoncer sa femme au travail. Lorsque, au contraire, le ménage est bien tenu par une femme qui reste au foyer et ne travaille pas, mais que la situation financière est plus précaire, on voit le mari songer plus volontiers au travail rémunéré de sa femme, surtout dans les jeunes ménages en train de s'installer ${ }^{3}$. On voit donc que les réponses sont visiblement influencées par les situations concrètes.

Le fait que les maris qui approuvent le travail de leur femme soient plus nombreux parmi les ouvriers du textile que parmi les métallurgistes semble n'avoir rien d'étonnant. Ce qui peut nous surprendre, c'est plutôt que les attitudes des maris diffè-

Un autre symptôme de l'incohérence des opinions et des attitudes apparaît dans le désir, généralement exprimé, des parents d'assurer à leurs filles une instruction souvent supérieure à une simple formation professionnelle : ce désir n'est pas compatible avec une opinion défavorable au travail des femmes mariées.

2 On trouve aussi quelques considérations sur l'effet négatif ou positif du travail sur la personnalité de la femme. Les premières sont plus fréquentes que les secondes.

3 Cela ne signifie pas que l'accroissement du revenu familial puisse, à lui seul, freiner l'activité professionnelle des femmes. Il faut voir là l'effet d'une multitude de facteurs, parmi lesquels le désir d'un niveau de vie toujours plus élevé n'est pas le moins important. 
rent si peu, malgré la grande différence qui sépare les traditions professionnelles des deux milieux, notamment en ce qui concerne le travail des femmes mariées. Il est non moins intéressant que des femmes mariées, ouvrières du textile, aient une attitude moins positive à l'égard du travail que des femmes mariées à des métallurgistes.

\section{L'attitude des femmes mariées envers le travail}

$\underline{\text { Retour à la table des matières du } 2^{\mathrm{r}} \text { article sur les Polonaises }}$

Dans les familles ouvrières de Varsovie, comme dans celles de Lodz, les femmes qui expriment le désir de continuer à travailler ou d'entreprendre de le faire sont plus nombreuses que ne semblent le souhaiter leurs maris.

Parmi les ménages interviewés à Varsovie, 26 maris seulement ont exprimé le désir de voir travailler leur femme, tandis que 56 femmes voudraient avoir un emploi, A Lodz, 25 maris voudraient que leur femme travaille, 28 femmes voudraient travailler. Les réponses des femmes ne sont pas strictement comparables à celles des maris, du fait que les questions adressées aux maris avaient été formulées un peu différemment. Cependant une analyse plus détaillée des réponses, tenant compte du contexte des autres réponses recueillies au cours des interviews, démontre que souvent - et, le plus souvent, dans le milieu de Varsovie - la femme voudrait travailler, en dépit de l'attitude de son mari. À Varsovie, dans la plupart des cas (29 sur 56), la femme voudrait travailler, mais le mari ne le veut pas. Ce fait se remarque surtout dans les ménages les plus âgés. Le plus souvent, c'est dans les jeunes ménages, désireux d'améliorer leur situation financière par un effort commun et un travail rémunéré des deux époux, qu'on voit ceux-ci se mettre d'accord pour que la femme trouve un emploi aussitôt qu'elle pourra régler la question des soins à donner à ses enfants.

Dans les attitudes des femmes mariées envers le travail salarié on peut observer le même phénomène que dans les attitudes des maris : la plupart des épouses qui travaillent voudraient renoncer à leur travail, tandis que la plupart de celles qui ne travaillent pas voudraient le faire. Cela se remarque tout particulièrement dans les familles des ouvriers du textile, où, sur 69 femmes mariées qui travaillent, 3 seulement voudraient continuer à le faire. Des 33 femmes mariées qui ne travaillent pas, 25 voudraient trouver un emploi.

Dans les réponses obtenues, le désir de renoncer au travail n'est formulé qu'à la condition «que la situation de la famille le permette ». Une femme mariée exprimant ce désir ne considère donc pas nécessairement une telle perspective comme vraiment possible. Il faut attribuer une signification analogue au désir de trouver un emploi, exprimé par des femmes mariées qui ne travaillent pas. 
Il est caractéristique que la différence la plus marquée entre le milieu de Varsovie et celui de Lodz se manifeste dans les attitudes des femmes qui travaillent. A Varsovie, environ $50 \%$ des épouses, surtout parmi les jeunes (du fait des enfants en bas âge) voudraient cesser de travailler. Presque toutes expriment ce désir à Lodz. Ce désir (ou peut-être ce rêve ?) de renoncer au travail paraît étonnant chez les ouvrières du textile : il semble peu réalisable dans ce milieu où tarit de femmes mariées travaillent depuis des années. Le désir des mères d'abandonner leur travail semble être plus vif à mesure que la nécessité de travailler devient plus accentuée pour des raisons économiques.

Des trois milieux considérés, c'est celui du textile où les maris gagnent relativement moins. Les revenus des femmes, n'étant pas de beaucoup inférieurs - ou étant même égaux - à ceux des hommes, ont une plus grande influence sur la situation financière de la famille. Les métallurgistes et, naturellement, les mineurs, ayant des salaires très élevés, les sommes que peuvent gagner leurs femmes ne peuvent être que relativement modiques et n'ont pas une telle importance pour le niveau de vie de la famille. Les différences d'attitudes envers le travail des femmes jeunes et des femmes, plus âgées sont plus marquées dans les familles de métallurgistes de Varsovie. Parmi les femmes qui travaillent, celles qui veulent continuer à le faire sont, pour la plupart, des femmes âgées, tandis que, parmi celles qui ne travaillent pas, ce sont, en majorité, des jeunes qui désireraient travailler. On ne trouve pas ces différences d'opinion parmi les ouvrières du textile.

En considérant les motifs (voir tableau, p. 85) qui, selon les réponses des femmes, les inclinent à effectuer un travail salarié ou le leur font désirer, il convient de constater que c'est le désir de gagner de l'argent pour la famille qui domine visiblement. Tous les autres motifs, d'ordre plus personnel, y compris le désir d'avoir de l'argent bien à soi, cèdent le pas à ce motif d'ordre économique et familial. Ce phénomène se remarque plus distinctement parmi les femmes mariées de Lodz. Il faut souligner que, lorsque les femmes répondent «plus d'argent pour le ménage », elles pensent souvent «plus d'argent pour les enfants ». Les besoins des enfants, le désir de leur assurer une vie meilleure, sont un motif très important qui encourage les femmes à travailler.

Le désir d'avoir «plus d'argent pour le ménage » a été le seul motif avancé par presque toutes les femmes qui effectuent un travail salarié et qui auraient désiré abandonner ce travail, si les revenus de leur mari s'amélioraient sensiblement. D'autres motifs, notamment le désir d'atteindre une certaine indépendance personnelle et d'avoir des contacts sociaux, sont avancés plus fréquemment par les femmes qui désirent travailler et par celles qui ne travaillent pas. A Varsovie, une différence assez marquée a pu être notée entre la motivation des femmes mariées, selon qu'elles étaient plus âgées ou plus jeunes. Les plus âgées soulignent plus fortement que les plus jeunes que leur travail est motivé par des raisons autres que l'aide financière qu'elles apportent au ménage. Pour les Varsoviennes du groupe le plus âgé qui ne travaillent pas, la nécessité de contribuer aux ressources du ménage cède le pas à d'autres motifs. 


\section{Quelques conclusions}

$\underline{\text { Retour à la table des matières du } 2^{\mathrm{r}} \text { article sur les Polonaises }}$

Les observations faites lors de l'étude présentée ci-dessus exigent d'être vérifiées par des recherches plus vastes et plus approfondies. Elles n'en inclinent pas moins à formuler certaines hypothèses et certaines conclusions.

1. Lorsque le pattern traditionnel devient désuet, en fonction d'un processus inexorable de changements sociaux et économiques, il en résulte que la manière d'agir (behaviour) se rationalise et s'individualise. La place et le rôle de la femme ne sont plus déterminés par les coutumes. Le processus de rationalisation est accompagné d'une incohérence et d'une diversité d'attitudes à l'égard des femmes mariées qui travaillent, tout particulièrement à l'égard de la propre épouse du sujet interrogé. On observe également une ambivalence de jugements et d'opinions. On voit les sujets interrogés peser les raisons qui peuvent être avancées pour et contre le travail des femmes mariées.

2. Les attitudes des maris envers le travail des femmes mariées sont plus conservatrices que celles de leurs épouses. Mais, là encore, ces attitudes ne semblent pas être fondées sur l'opposition à l'effondrement des coutumes traditionnelles. La source en paraît être l'inquiétude des maris en ce qui concerne leur propre situation dans la famille, jointe à des considérations utilitaires. L'aspiration des femmes mariées à entreprendre un travail salarié hors de leur domicile semble être plus générale que la volonté des maris d'accepter cette aspiration.

3. Les mères mariées assument le rôle de soutien - ou plus souvent de cosoutien de la famille. Dans la conscience d'une mère mariée, ce rôle se rattache principalement au souci du bien des enfants.

4. Les attitudes des femmes mariées envers le travail salarié, ainsi que les motifs avancés en faveur de ce travail, semblent dépendre de la situation particulière de la famille et du degré de développement qu'elle a atteint. Ils sont différents pour les jeunes mariées, dont la famille se développe, et pour les épouses plus âgées, dont les enfants sont devenus des adultes qui quittent la famille.

5. Bien que le motif économique («l'argent pour le ménage ») domine sensiblement, d'autres motifs ne sont pas moins mis en évidence. Ils sont d'autant plus soulignés que les besoins dont ils découlent sont moins satisfaits (par exemple : le désir de s'émanciper et de nouer des contacts sociaux, chez les femmes mariées qui ne travaillent pas), et que les nécessités économiques sont moins pressantes (par exemple, dans les familles les plus âgées des métallurgistes de Varsovie). 
6. Les réponses recueillies dans les interviews sont assez fortement conditionnées par la situation de la personne interviewée. Les désirs et les besoins non satisfaits se manifestent plus distinctement dans les opinions et les attitudes - et sans doute aussi dans la conscience - des intéressés que les désirs et besoins qu'ils ont déjà pu satisfaire. Cela ne veut pas dire que ces derniers jouent un rôle moins important dans la détermination des attitudes et, par suite, des actions. Un changement de situation peut entraîner des modifications considérables dans les opinions, les jugements, l'orientation et la motivation de la même personne.

Il subsiste, enfin, un doute d'ordre général : Peut-on considérer que l'étude présentée dans cet article ait vraiment révélé les «attitudes » à l'égard du travail des femmes ? Le terme « attitude » fait songer à certaines tendances, à certaines dispositions, à une manière d'agir définie. Cependant, nos recherches ont fait apparaître que les opinions relatives au travail des mères mariées, que nous avons recueillies dans les familles étudiées, étaient : a) contraires à la réalité existant dans ces familles (un exemple frappant en est fourni par les ouvriers du textile, dont la majorité se prononcent contre le travail de leurs femmes, bien que la plupart de celles-ci travaillent, pour ainsi dire, par tradition); $b$ ) incohérentes et changeantes (par exemple : le mari est content que sa femme travaille, mais il voudrait qu'elle y renonce; ou bien des femmes qui travaillent voudraient abandonner leur emploi et, au contraire, celles qui ne travaillent pas, voudraient en trouver un ; c) contradictoires, selon qu'il s'agissait de femmes de la même catégorie qui travaillent ou ne travaillent pas. 


\section{III \\ Femmes et professions au Maroc}

Au Maroc, un groupe de jeunes chercheurs marocains et français ont pu travailler en commun à une première enquête pilote. Mlle Forget présente ici une partie des résultats obtenus. Le temps a manqué pour permettre à plusieurs membres de l'équipe de s'exprimer comme nous l'aurions désiré. Seule Mlle Khadidja Nouacer a pu donner son point de vue et ajouter des remarques importantes aux données recueillies sur le terrain. D'autres chercheurs, pris par leurs travaux universitaires, ont demandé un délai pour apporter leur contribution. Ainsi en est-il, par exemple, de Mme Wassinck-Graef, assistante à la faculté des lettres de Rabat, de M. Khatibi, de Mlle Fatima Mernissi, de Mlle K. Brotzkis et d'autres jeunes sociologues qui se préparent à poursuivre, au Maroc, des études qui, dans l'avenir, seront du plus haut intérêt pour les comparaisons internationales.

I. Nelly Forget, “Attitudes à l'égard du travail professionnel de la femme au Maroc"

II. Khadidja Nouacer, "Évolution et travail professionnel de la femme au Maroc" 
III

Femmes et professions au Maroc

\section{I. \\ "Attitudes à l'égard du travail professionnel de la femme au Maroc" par Nelly Forget,}

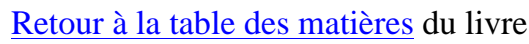

Table des matières du $1^{\text {er }}$ article sur les femmes au Maroc

Introduction

1. Méthodologie

2. Choix des métiers
a) Le travail à domicile

3. Critiques, rejets et restrictions
a) La promiscuité
b) Perturbations dans le monde du travail
c) Manque d'aptitudes et de formation
d) Répercussions familiales
e) Bouleversement des rôles sociaux

4. Motivations
a) Les besoins économiques familiaux
b) Décalage entre les modèles et les comportements
c) La notion d'utilité sociale
d) L'intérêt personnel de la femme

Bibliographie 


\section{Introduction}

$\underline{\text { Retour à la table des matières du 1er article sur les Marocaines }}$

Cette brève étude s'insère dans l'enquête internationale, dont les principes et les modalités ont été exposés plus haut ${ }^{1}$.

Répondant aux mêmes préoccupations que les recherches menées dans d'autres pays, elle adopte le même point de vue : essayer de dégager les attitudes à l'égard de la condition de la femme dans le Maroc d'aujourd'hui, en insistant particulièrement sur ce qui a trait au travail professionnel, et en se limitant à ce qui est observable en milieu citadin.

Dans un pays en pleine transformation comme le Maroc, il ne saurait être question de dissocier le problème du travail des autres aspects fondamentaux du statut féminin et des bouleversements de la société globale. Il est évident, même pour l'observateur non averti, que la claustration, la survivance des structures patriarcales, l'indépendance nationale, l'urbanisation et la naissance du prolétariat - pour ne citer que quelques faits - et les jugements qu'on porte sur ces phénomènes, déterminent, en grande partie, les attitudes qu'on adopte à l'égard du travail professionnel de la femme.

C'est pourtant ce dernier problème - et seulement celui-là -que nous allons étudier ici, nous réservant d'aborder, dans une étude ultérieure, les autres aspects de l'évolution féminine.

Qu'on ne s'attende donc pas à trouver ici un exposé des situations de fait - mises à part quelques références incidentes, destinées à illustrer certaines attitudes. Plutôt qu'avec les structures nouvelles ou anciennes, dont il serait, bien entendu, très intéressant de faire le bilan, nous essaierons de faire connaissance avec les diverses réactions qu'engendre la situation évolutive du pays, d'analyser l'interpénétration des modèles traditionnels et des nouveaux idéaux, de préciser ce qui, dans les mentalités originales et souvent conflictuelles qui en sont issues, constitue des aspirations dynamiques ou, au contraire, des zones de freinage.

En tout ceci, la présente étude obéit aux mêmes préoccupations que les enquêtes menées en France et en Pologne sur les attitudes à l'égard du travail de la femme. Elle ne peut, cependant, leur être comparée, dans la mesure où elle ne prétend pas à la même rigueur scientifique et aux mêmes résultats qu'une véritable enquête. Il s'agit, en fait, d'un essai d'observation, reposant sur l'étude d'une série de cas regroupés suivant des situations socio-économiques caractérisées, d'une étude typologique préliminaire à une enquête systématique.

1 Voir p. 13 de l'édition papier de la revue. Voir, dans cette édition numérique : Introduction : 2. 
Il est nécessaire de bien préciser les limites de ce travail préliminaire, pour éviter tout malentendu sur sa portée (en particulier, on ne doit pas accorder plus de valeur qu'ils n'en ont aux pourcentages cités pour illustrer un certain nombre d'observations qualitatives). Le peu de temps disponible et la médiocrité des moyens mis en oeuvre expliquent que, pour le moment, l'étude n'ait pas dépassé le stade préparatoire. Les critiques que l'on peut formuler à l'encontre de la méthode suivie peuvent, à notre avis, être regroupées en trois points ${ }^{1}$.

En premier lieu, l'échantillon de population interrogé n'a pas été constitué selon des normes rigoureuses, permettant d'isoler des variables indiscutables, et il ne peut être considéré comme représentatif de catégories précises de la population citadine. La seconde difficulté est d'ordre linguistique. Le questionnaire de la présente enquête a été présenté en français aux sujets bilingues, et traduit oralement en arabe dialectal aux sujets qui ne parlaient pas français. En troisième lieu, on peut se demander si les techniques d'enquête par questionnaire, qui ont fait leurs preuves dans les pays d'Europe occidentale, peuvent être appliquées telles quelles en pays arabe. Peuventelles l'être, d'ailleurs, valablement par une étrangère à double titre, non musulmane et non marocaine ?

L'expérience tentée apporte quelques éléments de réponse à cette dernière question, qui reste cependant posée. A aucun moment, nous ne nous sommes heurtés à des refus de répondre, ni même à des réticences. Sans vouloir négliger ce qui, dans cette attitude, peut être attribué à l'extrême courtoisie des Marocains et à leur sens de l'hospitalité, qui peuvent recouvrir des réserves profondes, nous pouvons, cependant, prétendre qu'au cours des entretiens d'une ou plusieurs heures que nous avons eus avec chacun des sujets interrogés, il aurait été impossible de ne pas déceler les refus de rapports et les réticences.

Nous devons la qualité de cet accueil, en premier lieu, à l'aide apportée par les Marocains et les Marocaines qui nous ont introduite dans des milieux divers - et il convient ici de remercier tous ceux qui ont bien voulu nous aider dans ce travail : la Direction de la jeunesse et des sports, qui nous a permis de faire appel à la collaboration précieuse du personnel des foyers féminins, les directions des écoles d'infirmiers et d'infirmières, et des stages du Ministère du travail, la Circonscription de l'urbanisme et de l'habitat, le Service de la statistique, la direction des usines Tezier, et les jeunes étudiantes marocaines, ainsi que les jeunes femmes françaises et les instituteurs qui, à titre individuel, ont participé à l'enquête.

Un autre facteur a joué en notre faveur : l'actualité du sujet traité. Le problème de la femme est central. Tout le monde a, sur ce problème, une opinion, souvent passionnée. Tout le monde en discute; notre enquête n'était, pour la plupart des sujets interrogés, que le prolongement de discussions récentes ou l'occasion d'exprimer des préoccupations personnelles, qui sont réelles et profondes. Plusieurs directeurs d'établissement scolaire ou de stage nous ont signalé que, dans les discussions libres, le thème choisi spontanément était presque toujours relatif à la femme. Au cours d'une récente conférence à l'Institut de psychologie sociale de Paris, où il présentait les premiers résultats d'une étude sur l'influence des variables socio-culturelles sur un

1 Critiques qui appelleraient quelques mises au point que nous n'avons pas la place de développer ici. 
groupe de jeunes au Maroc ${ }^{1}$, J. Selosse indiquait qu'en tête des 27 préoccupations communes à son échantillon, se détachait le problème de la situation de la femme.

Ce n'est d'ailleurs pas un débat théorique ; les problèmes soulevés par le travail de la femme se posent à un nombre grandissant de familles. Les statistiques font apparaître, en effet, que les femmes qui travaillent ne sont plus des exceptions. Le recensement de 1952 dénombrait 160000 femmes qui se répartissaient comme suit dans les divers secteurs d'activité ${ }^{2}: 85000$ dans l'artisanat et l'industrie, Soit $27 \%$ de la main-d'œuvre totale employée dans cette branche d'activité ; 46000 dans les soins personnels et les services de santé, soit 64\%; 22000 dans la manutention et les transports, soit $8 \%$; 3000 dans le commerce, soit 2\%: 1800 dans l'administration et les emplois intellectuels, soit $2 \%$.

Suivant un autre mode de répartition, la main-d'œuvre féminine (toujours en 1952) aurait été utilisée de la façon suivante : $30 \%$ de domestiques, dans des maisons européennes; $20 \%$ d'ouvrières d'entreprise industrielle; $20 \%$ de main-d'œuvre saisonnière; $30 \%$ occupant divers emplois (13\% dans le travail de la laine [filage, tissage], $7 \%$ de couturières, $4 \%$ dans le commerce [surtout le petit commerce), $1 \%$ dans les professions libérales [infirmières, monitrices, maîtresses d'école, etc.], enfin $4 \%$ occupant divers emplois indéfinissables).

En rapprochant ces chiffres de la population en âge de travailler, on constate qu'environ une femme sur 8 travaille $[4, \mathrm{p} .41]^{3}$. La proportion diffère suivant les milieux : elle est beaucoup plus élevée dans les milieux populaires [I, p. 134 ; II, p. $24 ; 13$, p. 224] ${ }^{4}$. Elle est surtout en pleine évolution, car les filles vont de plus en plus nombreuses à l'école et s'y préparent à exercer une profession.

Jusqu'en 1944, les effectifs scolaires féminins ont stagné, comme d'ailleurs les effectifs masculins (1939 : 5300 filles scolarisées ; $1944: 10$ 300). À partir de cette date, on assiste à une poussée, encore faible $(1945: 10,300 ; 1950: 23500 ; 1954: 55$ 500 ), mais régulière (de l'ordre de $15 \%$ par an), Mais le vrai démarrage a lieu au lendemain de l'indépendance $(1956$ : $92500 ; 1957$ : 138 000; 1959 : environ 180 000). Dans un collège technique de Casablanca, en 1958, 15 Marocaines entraient en classe de troisième de l'enseignement secondaire, en 1959, 53, et l'on en prévoyait 75 pour la rentrée de 1960. Dans le même établissement, dans la classe de fin d'études commerciales, on comptait 15 Marocaines en I958-1959, et 37 en I959-I960. En 1953, 12 femmes avaient bénéficié d'une formation pédagogique dans un établissement spécialisé ; en 1960, le chiffre correspondant était de 148.

Notons, cependant, que la progression des effectifs scolaires féminins s'inscrit dans un mouvement général, sans que le pourcentage des filles ait beaucoup augmenté dans l'ensemble de la population scolaire (1944:20\%;1950:20\%; $1954: 23 \%$;

En cours de publication dans le Bulletin de la Société française de Psychologie.

Non compris les 771000 femmes concourant à la production agricole (753000 non salariées et 18000 salariées).

Les chiffres entre crochets renvoient à la bibliographie en fin d'article.

D'après R. Montagne, 50\% des femmes en milieu prolétarien travaillent. D'après A. Adam, un sondage portant sur 187 femmes qui habitent le bidonville de Ben Msick, à Casablanca, établit que $47,6 \%$ d'entre elles ont une activité professionnelle. Mathieu et Maneville, dans un autre sondage fait aux Carrières centrales, à Casablanca, et portant sur 73 familles, dénombrent 46 femmes qui travaillent. 
1959 : 26\%) ${ }^{1}$. Mais la progression des chiffres absolus nous paraît beaucoup plus importante que celle des pourcentages. En s'ouvrant aux masses citadines et rurales, l'école, réservée auparavant à quelques milliers de privilégiées ou d'audacieuses, est en train d'opérer une mutation brusque de la société féminine - et de la société globale marocaine. «Un enfant scolarisé sur 20, cela suffit pour mettre en faillite l'éducation traditionnelle reçue par les 19 autres ; mais, si les 20 enfants trouvent place dans une école moderne, ils pourront tenir le coup dans une société moderne, tous les $20 »[17$, p. 631. Bien sûr, en ce qui concerne les filles, elles ne sont pas encore «toutes les 20 » à l'école, mais il y en a beaucoup plus d'une sur 20, et les profonds changements qui en découleront immanquablement seront perceptibles dans quelques années, lorsque la masse des petites filles qui s'assoient aujourd'hui sur les bancs de l'école seront devenues des femmes.

\section{Méthodologie}

$\underline{\text { Retour à la table des matières du 1er article sur les Marocaines }}$

Nous ne traiterons ici que de l'enquête par questionnaire, bien qu'elle ne représente qu'un des moyens mis en œuvre -l'interview libre de personnalités, les observations de caractère ethnographique et la recherche de documents ayant joué un rôle au moins aussi important dans nos travaux.

Le questionnaire a été mis au point en prenant pour base celui qui avait été établi pour l'enquête française par M. P. Chombart de Lauwe et le Groupe d'ethnologie sociale. Après des adaptations successives qui ont été mises à l'épreuve dans différents milieux, une rédaction définitive a été établie. Elle reste assez proche du document utilisé en France pour ce qui ressortit aux problèmes professionnels, mais elle s'en éloigne fortement pour les questions relatives au mariage et à la vie familiale. Le questionnaire est rédigé en français. Sa traduction en arabe dialectal a été enregistrée, de façon que les différents interprètes utilisent les mêmes mots clés.

Comme nous l'avons déjà indiqué, l'échantillon n'a pu être constitué suivant des normes rigoureuses. Dans la perspective qui était la nôtre dès le départ - celle d'une enquête pilote - il nous a paru préférable de multiplier les terrains d'enquête, de nous adresser à des milieux aussi divers que possible, de façon à disposer d'un éventail de cas aussi large que nous le permettaient les circonstances et les introductions dont nous bénéficiions. Nous n'avons pas tenu compte de la règle appliquée dans la recherche française, où l'homme et la femme d'un même couple étaient interrogés conjointement : pour des raisons pratiques, ce procédé aurait singulièrement compliqué notre tâche, et réduit encore le nombre des interviews.

Tous ces chiffres sont tirés des tableaux statistiques du Ministère de l'éducation nationale (non publiés). 
Les enquêtes menées à Rabat, Fès et Casablanca ont permis de réunir 63 questionnaires, se répartissant comme suit

D'après le sexe (35 femmes et 28 hommes).

D'après la situation matrimoniale (célibataires : femmes, 12; hommes, 19 ; mariés ${ }^{1}$ : femmes, 23; hommes, 9). Parmi les femmes mariées $22 \%$ n'avaient pas d'enfant, 22\% avaient 1 enfant, $13 \% 2$ enfants, $17 \% 3$ enfants, 22\%, 4 enfants et $4 \%$ plus de 4 enfants.

D'après l'âge. La variable « situation matrimoniale » recouvrait la variable âge : en effet, chez les femmes, les célibataires avaient toutes moins de 21 ans (moyenne : 17 ans et demi), et les femmes mariées de 20 à 43 ans (moyenne : 28 ans). Chez les hommes, les célibataires avaient entre 18 et 27 ans (moyenne : 21 ans), et les hommes mariés, entre 23 et 40 ans (moyenne : 31 ans).

D'après le milieu socio-économique. Femmes (12 ouvrières, dont Ici travaillant en usine, à Fès ${ }^{2}$; 13 fonctionnaires appartenant aux cadres moyens [santé ou enseignement] ; 10 femmes au foyer, dont 8 appartenant à la bourgeoisie de Fès, et 2 travaillant occasionnellement chez elles). Hommes ( 2 ouvriers de l'industrie ; 22 fonctionnaires de rang moyen [santé, travail, justice et intérieur] ; 3 élèves des grandes classes ; 1 commerçant).

La présentation des critiques qui s'expriment contre le travail de la femme et qui aboutissent soit à un rejet pur et simple, soit à des restrictions, précédera le bilan des attitudes positives et des motivations qui conduisent à accepter avec des nuances très différentes, allant de l'enthousiasme à la résignation, l'accès de la femme à la vie professionnelle, avec toutes ses implications familiales et sociales. Mais, auparavant, le problème sera abordé d'une façon aussi concrète que possible, par l'étude du choix des métiers.

Nous avons compté au nombre des femmes mariées les femmes divorcées ou veuves, cette situation étant temporaire, car les remariages sont très fréquents.

2 Nous aurions souhaité pouvoir établir la comparaison avec un échantillon de même catégorie socio-professionnelle, pris dans une autre ville, Casablanca par exemple. Les circonstances ne l'ont pas permis. Nous le regrettons beaucoup, car, dans les réactions fortement caractérisées des ouvrières, il est difficile de démêler ce qui revient à l'influence de la ville de Fès, dont la tradition puissante et originale n'est pas sans rayonner sur les bidonvilles de la périphérie. 


\section{Choix des métiers}

$\underline{\text { Retour à la table des matières du 1er article sur les Marocaines }}$

L'enquête proposait une liste de 15 métiers ${ }^{1}$, qui sont effectivement exercés par des femmes dans le Maroc actuel : métiers traditionnels ou modernes, demandant ou non une qualification professionnelle, donnant droit à un salaire médiocre ou relativement élevé, ressortissant au genre de vie traditionnel ou à l'économie moderne.

Parmi les métiers appartenant à cette dernière catégorie, on trouve les suivants :

Secrétaire. Profession en pleine expansion, puisque la marocanisation accélérée des administrations et des entreprises privées augmente considérablement les offres d'emploi et, partant, le niveau des salaires ${ }^{2}$.

Institutrice. La croissance des effectifs ${ }^{3}$ manifeste le prodigieux développement de cette profession ; elle ne risque pas d'être saturée de sitôt, étant donné les immenses besoins que suppose la scolarisation d'une jeunesse toujours plus nombreuse.

Ouvrière d'usine. Les femmes appartenant à cette catégorie exercent leur activité dans l'industrie du textile, mais, plus encore, dans les industries de transformation des produits alimentaires : conditionnement des agrumes, fabrication de pâtes, de couscous, conserves de fruits, de légumes, de poisson, etc. Le travail, saisonnier le plus souvent, peut s'échelonner sur quelques semaines ou sur plusieurs mois de l'année, ce qui ne va pas sans entraîner de graves déséquilibres familiaux et sociaux (migrations temporaires, désintégration familiale, mariages saisonniers eux aussi, etc.). Les statistiques de 1952 dénombrent près de 10000 femmes employées dans les métiers relevant de l'alimentation et plus de 15000 dans les secteurs de la manutention. Les métiers relevant du textile et de l'habillement groupent 70000 femmes, sans que les chiffres donnés permettent de différencier les innombrables fileuses, brodeuses, couturières, etc., à domicile de celles qui sont employées dans des ateliers semiindustriels.

À cette première liste s'ajoutent les professions suivantes : infirmière, sagefemme, fonctionnaire (cette dernière choisie en vue de faire ressortir l'intérêt attaché

Secrétaire, domestique, porteuse d'eau, institutrice, ouvrière d'usine, vendeuse de magasin, gardienne de hammam, moallema (maîtresse de broderie), sage-femme, artiste de théâtre, fonctionnaire, infirmière, commerçante, brodeuse, serveuse de restaurant.

2 La directrice d'un collège technique de Casablanca nous signalait que les élèves de la section commerciale, sollicitées par les employeurs, abandonnent souvent leurs études en cours d'année, sans avoir besoin d'attendre leur diplôme, pour gagner immédiatement des salaires s'échelonnant de 35000 à 45000 anciens francs par mois.

3 Dans le personnel de l'enseignement primaire, on comptait 54 Marocaines en 1947, 98 en 1954, 819 en 1958, 2352 en 1960, dont 786 « qualifiées » et 1566 non qualifiées (enquête de l'Unesco). 
au salaire et à la stabilité de l'emploi, indépendamment des fonctions remplies), vendeuse de magasin, serveuse de restaurant.

Parmi les métiers traditionnels, étaient cités les suivants :

Brodeuse. Cette activité est l'une des plus fréquemment exercées par les femmes qui restent chez elles. La broderie fait partie de la formation traditionnelle de toute jeune fille citadine : art d'agrément pour les plus fortunées, elle offre des débouchés dans les innombrables productions de l'artisanat (linge de maison, babouches, cafetans, etc.). La rémunération reste très faible ${ }^{1}$.

Porteuse d'eau. On trouve des représentantes de cette corporation surtout dans les bidonvilles, où la rareté des points d'eau oblige à de longs parcours et à de longues attentes [I, p. 134 ; II, p. 24 ]

Gardienne de hammam. Préposée à la gestion du bain, qui est, par excellence, le foyer des relations sociales féminines, où se colportent les nouvelles, où se concluent bien des mariages, la gardienne, au carrefour de tous ces échanges, est souvent amenée à s'entremettre dans la vie des familles. Quelques autres professions sont ambivalentes, dans la mesure où elles peuvent revêtir des aspects différents, tantôt modernes, tantôt traditionnels.

Telle la commerçante, qui peut tenir boutique, mais qui peut également être colporteuse de tissus et de nouvelles, entremetteuse tout autant que marchande.

Telle l'artiste : elle est ou bien danseuse anonyme (toujours réputée de mœurs légères) d'une troupe traditionnelle - et l'on fait appel à elle pour rehausser les festivités familiales (mariage, circoncision), - ou bien vedette du disque et du film, universellement connue de Bagdad à Rabat - et elle appartient alors à l'univers des stars, mais aussi, quasiment, à celui des héros nationaux. C'est elle, en effet, qui remet à la disposition de chacun, grâce aux moyens de communication modernes, un patrimoine culturel d'expression arabe, en même temps qu'elle vulgarise et renforce de nouvelles façons de sentir, de nouvelles aspirations, de nouveaux modèles sentimentaux $[5,13-170]$.

Telle encore la domestique, dont la condition varie beaucoup suivant l'employeur. Bonne à tout faire salariée, chez les Européens, elle est plutôt intégrée dans les familles marocaines, avec tous les avantages et les contraintes que suppose ce statut de quasi-adoption. Elle peut également être employée dans une entreprise ${ }^{2}$.

Les sujets interrogés devaient répondre à la question : « Est-ce bien ou mal pour une femme de faire tel métier? » (ceci pour chacune des 15 professions énumérées).

Les ouvrières ont répondu positivement pour presque tous les métiers (à l'exclusion de celui de serveuse de restaurant et de celui d'artiste), avec ce commentaire qui revient souvent : «Pourvu qu'il donne de quoi vivre, tout travail est bon. » Une

Voir p. 102 de l'édition papier de la revue. Dans l'édition numérique, voir la section 2a.

2 Voici, à titre indicatif, l'horaire de femmes de ménage employées à l'entretien des bureaux à Casablanca (les bureaux sont situés au centre de la ville, tandis que les femmes de ménage résident dans les bidonvilles de la périphérie) : lever vers 3 heures du matin ; 8 kilomètres à pied pour gagner le centre de la ville ; travail de 5 heures à 9 heures; retour à la maison; travail de 18 à 20 heures quatre trajets dans la journée, toujours à pied, soit une trentaine de kilomètres). 
femme peut faire tel ou tel métier «si elle n'a pas d'autre ressource », « uniquement parce que c'est mieux que de mendier », « mieux que de laisser ses enfants mourir de faim $\gg$.

Le reste de l'échantillon féminin exprime des opinions à la fois plus nuancées car tous les métiers ne bénéficient pas de la même approbation massive et indifférenciée - et plus fermes, dans la mesure où les choix positifs sont moins souvent assortis de restrictions. Il en va de même pour les hommes; la hiérarchie et le pourcentage de leurs options sont tout à fait comparables à ceux des femmes non ouvrières, mises à part quelques légères différences (les métiers de brodeuse et d'ouvrière d'usine sont plus prisés par les femmes, tandis que celui d'artiste de théâtre est mieux accepté par les hommes).

Ces choix communs font ressortir trois grandes catégories de métiers :

1. Ceux qui sont fréquemment rejetés : commerçante (54\% de rejets), gardienne de hammam, artiste, domestique (entre $60 \%$ et 70 porteuse d'eau, serveuse de restaurant (plus de 90\%).

2. Un second groupe de professions est largement choisi (entre $65 \%$ et $75 \%$ de choix positifs) : fonctionnaire, maallema (maîtresse de broderie), vendeuse, ouvrière d'usine ( $65 \%$ chez les femmes, $42 \%$ chez les hommes).

3. Enfin, ralliant plus de $90 \%$ des suffrages, plusieurs professions sont au-dessus de toute critique : brodeuse (100\% chez les femmes, $65 \%$ chez les hommes), institutrice, sage-femme, infirmière, secrétaire.

Mise à part la brodeuse, qui représente l'image traditionnelle de la femme au foyer, il s'agit de professions nouvellement introduites au Maroc, qui s'inscrivent dans le cadre de l'économie moderne ; elles supposent une scolarisation poussée, s'exercent en dehors du domicile et représentent une rupture avec les modes de vie traditionnels. Cependant, sont-elles si éloignées du rôle traditionnellement imparti à la femme ? Elles rallient, en tout cas, à elles les plus fermes adversaires de l'émancipation féminine, si l'on se réfère à un arrêt des docteurs d'Al Ahzar qui, dans une fetwa ${ }^{1}$ du 11 juin 1952 [15, no 3, mars 1956], relative aux droits et devoirs de la femme, après avoir dénié à celle-ci le droit d'accéder à des fonctions publiques, « à cause de sa féminité qui l'expose à sortir des voies de la raison et de la mesure », admettent cependant qu'elle exerce certaines professions « où la force du jugement et de la volonté ne se déploie pas » (par exemple : professeur pour jeunes filles, médecin ou infirmière s'adressant à une clientèle féminine).

Pour de tout autres raisons, plus positives en tout cas, la faveur dont jouissent ces professions se manifeste à propos d'une question incidente : «Est-ce que vous accepteriez aussi bien d'avoir affaire à une femme qu'à un homme dans tel métier ? » Des hommes, $20 \%$ seulement refuseraient de se faire soigner par une femme médecin. Encore est-ce par pudeur, la question de la compétence n'étant jamais mise en doute (sauf par $8 \%$ des femmes). C'est aussi la pudeur qui, dans bien des cas, conduit les femmes à préférer une autre femme : «Pas un homme, en tout cas, pour les maladies au-dessous de la ceinture », nous précise une femme qui a, effectivement, refusé un examen gynécologique urgent, parce qu'il aurait été pratiqué par un homme. Quant

Arrêt, décret. 
aux hommes, s'ils préfèrent une femme, c'est parce qu'elle est plus compréhensive, « parce qu'elle a un coeur doux ».

Dans les carrières de l'enseignement, les hommes accordent généralement une plus grande confiance à la femme qu'à l'homme : $82 \%$ accepteraient de confier leur fils à une institutrice, alors que 50\% seulement accepteraient qu'un instituteur s'occupât de leur fille. L'échantillon féminin semble se montrer plus conformiste ; sans faire preuve de défiance systématique à l'égard des représentantes de leur propre sexe (puisqu'elles leur donnent la préférence en ce qui concerne l'éducation des filles), les femmes paraissent satisfaites de la ségrégation traditionnelle (40 seulement acceptent que les rôles habituels soient intervertis, aussi bien pour l'institutrice que pour l'instituteur).

Est-ce conformisme, ou faut-il y voir un refus de se laisser cantonner dans un secteur d'activités restreint, de se laisser enfermer dans l'archétype de la femme maternelle, douce, compréhensive - archétype qui peut aussi apparaître comme une limite à dépasser ?

Est-il possible, à partir de cette liste de préférences, de définir les critères qui président aux choix et qui expriment des attitudes fondamentales à l'égard du travail professionnel de la femme?

L'intérêt pécuniaire est-il primordial ? La motivation économique est très forte, nous l'avons vu. Mais le métier de brodeuse, qui vient en tête des choix féminins, est l'un des moins rémunérés, alors que sont rejetées des professions beaucoup plus rentables, telles que celles d'artiste et de commerçante.

Le prestige dont jouit l'instruction détermine-t-il une orientation vers les professions qui exigent un niveau culturel relativement élevé ? Si, effectivement, les métiers rejetés ont en commun de ne pas nécessiter de titres particuliers, là encore, la place prééminente faite au métier de brodeuse et celle, relativement importante, qui est faite au métier d'ouvrière d'usine, contrediraient la valeur absolue de ce critère.

Est-ce l'attrait du modernisme qui joue ? S'il est vrai que les professions les plus fréquemment choisies appartiennent au secteur moderne, on ne peut oublier que certaines des professions de cette catégorie sont largement rejetées et que, dans le groupe de tête, on trouve également le métier de brodeuse.

Les préférences iraient-elles alors, sinon aux métiers traditionnels, du moins a ceux où, dans un cadre moderne, se perpétue une certaine image de la femme, où peut se transposer, dans la vie sociale, le rôle qui lui est habituellement dévolu dans une société patriarcale? C'est ce que laisserait supposer la faveur dont jouissent les carrières médicales et celles de l'enseignement, si l'on tient compte des restrictions (public ou clientèle exclusivement féminins) que préconisent les oulémas d'Al Ahzar. Mais, dans cette perspective, la secrétaire, soumise à tous les aléas de la promiscuité, ne devrait pas bénéficier de telles préférences.

Il est prématuré d'apporter une réponse à ce stade de la recherche. Bornons-nous, pour le moment, à constater la multiplicité des critères de choix et leur conjonction conflictuelle. Et poursuivons l'analyse, en examinant les positions adoptées à l'égard d'un problème complémentaire du choix des métiers : celui du travail à domicile. 
Cette notion met en cause la claustration de la femme et, au-delà, les modèles traditionnels.

\section{a) Le travail à domicile}

$\underline{\text { Retour à la table des matières du 1er article sur les Marocaines }}$

Les voix sont également partagées entre le travail à domicile (50\% chez les hommes, $57 \%$ chez les femmes) et le travail à l'extérieur. Mais les choix qui se portent sur le travail à domicile sont tempérés par des considérations économiques : de préférence à domicile, à condition que l'intéressée puisse y gagner un salaire suffisant ; d'aucuns ajoutent : « mais c'est rare ! ».

En effet, les salaires des travailleuses à domicile sont incomparablement plus bas que les autres. À Fès, une domestique gagne entre 300 et 500 anciens francs par jour (plus la nourriture); les ouvrières d'usine que nous avons interrogées reçoivent environ 460 anciens francs par jour (y compris les primes). Une travailleuse à domicile, qui consacre cinq à six heures à son ouvrage, peut rarement se faire plus de 150 anciens francs par jour. Il faut une journée de travail pour broder une paire de babouches : elle est payée 150 anciens francs ; une journée de travail pour broder sur tissu un écheveau de soie : le travail d'un écheveau est payé de 120 à 250 anciens francs, suivant le point de broderie et la finesse du tissu ${ }^{1}$. Quant aux fileuses, travaillant par équipe de deux, elles ne peuvent guère, en une journée, nettoyer, carder et filer plus d'un kilogramme de laine, qui leur est payé 15o anciens francs (soit 75 anciens francs par personne).

Encore s'agit-il là (à l'exception des fileuses) de ce que peut espérer gagner une ouvrière qualifiée, capable de réaliser des travaux raffinés et sans défaut - ce qui n'est possible qu'après un long apprentissage, durant lequel l'intéressée ne reçoit aucun salaire. Seules les maallemas (maîtresses de broderie) qui dirigent et commercialisent les travaux d'une équipe de femmes - ou, plus souvent, de petites filles, éternelles apprenties bénévoles peuvent prétendre à des gains satisfaisants.

Pour la majorité des sujets interrogés, la médiocre rentabilité économique du travail à domicile pèse plus lourd que ses avantages, qui sont cependant grands au regard de la tradition, puisqu'il permet à la femme de ne pas sortir. C'est parce qu'il procure plus d'argent que certains donnent leurs préférences au travail à l'extérieur. C'est encore une argumentation économique qu'utilisent ceux qui se refusent à faire un choix théorique : "Quand elle est obligée de travailler, il faut que la femme prenne le travail là où il se trouve. »

Malgré ce grave défaut, le travail à domicile n'en paraît pas moins enviable à de nombreuses ouvrières d'usine. À leur avis, ce n'est qu'en désespoir de cause qu'une femme va travailler à l'extérieur : «Si elle a reçu une éducation », « si elle a appris un métier », « elle peut travailler chez elle comme brodeuse ou couturière ».

1 Tarifs d'une coopérative de Fès, où les femmes sont avantagées par rapport a celles qui travaillent pour le compte d'une maallema. 
Pour les autres femmes, la proposition est exactement inverse : « Si une femme est instruite, qu'elle aille travailler à l'extérieur, elle recevra un bon salaire ». Sinon, sans éducation, elle ne pourra que rester chez elle et travailler comme brodeuse ou couturière.

Apparente contradiction, qui révèle deux niveaux sociaux différents, deux niveaux d'aspirations différents. L'instruction à laquelle aspirent les enquêtées signifie tantôt l'apprentissage manuel de plusieurs mois qui leur permettrait de s'affranchir des servitudes de l'usine, tantôt les années de scolarité nécessaires pour accéder à une profession moderne. Pour les ouvrières des bidonvilles, la promotion passe par la vie traditionnelle de la bourgeoise de la medina - carcan dont les autres estiment qu'il faut se libérer, lorsque la chance, c'est-à-dire l'instruction reçue, le permet.

En revanche, nous avons rencontré d'autres travailleuses, en particulier des domestiques, à Casablanca ${ }^{1}$, qui n'ont pas du tout la nostalgie du travail à domicile, mais sont, au contraire, très conscientes des avantages de leur situation : pouvoir sortir de la maison et échapper à l'emprise familiale, s'enrichir par des contacts, s'initier à de nouvelles façons de vivre et de faire les choses. Pour elles, la promotion rêvée consisterait non point à retourner au fuseau ou à l'aiguille, mais à acquérir des qualifications modernes (infirmière, par exemple).

Une autre question permettait de préciser le sens des aspirations, en projetant les choix vers l'avenir : «Quel métier aimeriez-vous que vos filles fassent plus tard ?»

On sollicitait d'abord une réponse spontanée, puis on proposait 19 métiers, qui reprenaient, en partie, ceux de la première liste, en y incluant d'autres professions qui, à l'heure actuelle, ne sont pas encore exercées par des femmes, au Maroc ${ }^{2}$.

Qu'il s'agisse des réponses spontanées ou de la liste précodée, les préférences exprimées confirment les choix précédents, en se portant, dans leur immense majorité, vers les professions médicales et paramédicales, et vers les carrières de l'enseignement. Deux professions d'avenir s'y ajoutent : avocate et ingénieur. Sont rejetés les métiers de domestique, de serveuse de restaurant, de coiffeuse, d'artiste, de commerçante, ainsi que la proposition de « rester chez soi et ne pas travailler » (8 à $10 \%$ seulement de personnes interrogées souhaitent ou envisagent la possibilité que leurs filles ne travaillent pas).

Cette orientation générale est commune aux hommes et aux femmes, mais il existe une notable différence dans les choix respectifs des deux sexes. Les hommes ont, pour leurs filles, des aspirations conformes à leurs options actuelles. L'ordre de leurs préférences et la fréquence de leurs choix sont identiques pour le présent et pour

Nous regrettons, une fois de plus, de ne pas disposer d'un échantillon d'ouvrières casablancaises, qui nous aurait permis d'isoler la variable « espace socio-géographique ». A priori, il semble que le poids et le charme des formes traditionnelles jouent à Fès un rôle attractif considérable, alors qu'à Casablanca les immigrants du bled sont directement mis en présence des modèles de la civilisation technique occidentale.

2 Les propositions étaient les suivantes : ne pas travailler, exercer une profession à domicile, infirmière, domestique, secrétaire, couturière, brodeuse, professeur de medersa, sage-femme, médecin, artiste de théâtre ou de cinéma, assistante sociale, fonctionnaire, coiffeuse, danseuse, avocate, institutrice, commerçante, ingénieur, serveuse de restaurant. 
l'avenir (à cette différence près qu'ils introduisent des métiers qui n'étaient pas cités dans la première liste : avocate, ingénieur, travailleuse à domicile).

En revanche, les femmes ne conçoivent pas que, dans l'avenir, l'échelle des valeurs soit exactement la même qu'aujourd'hui. La profession de couturière et brodeuse, par exemple, qui, actuellement, est approuvée à 100\% et vient au premier rang, ne bénéficie plus que de $8 \%$ des suffrages et vient au neuvième rang dans les professions que les femmes interrogées souhaitent pour leurs filles. De même pour le métier de secrétaire (94\% quatrième rang, et $8 \%$ neuvième rang). À l'intérieur des professions médicales, les femmes souhaitent une promotion, en donnant la préférence à la profession de médecin $(31 \%)$ sur celles d'infirmière $(20 \%$, de sagefemme et d'assistante sociale (14\%). Au contraire, les hommes, ne voyant aucune raison de bouleverser la hiérarchie actuelle, préfèrent que leur fille soient infirmière, assistante sociale (45\%), ou sage-femme (35\%), plutôt que médecin $(28 \%)$.

Cette tendance statique des hommes et évolutive des femmes se manifeste également à propos du travail à domicile. Lorsque la question, déjà abordée sous un angle général, leur est posée d'un point de vue plus personnel - «Souhaiteriez-vous que votre fille travaille à domicile ? » - les hommes restent à peu près tous fidèles à leur position de principe : $40 \%$ d'entre eux souhaitent que leur fille puisse travailler chez elle. Le travail à domicile leur paraît donc une solution durable, présentant des avantages solides, stables, bien adaptée aux exigences fondamentales de la vie familiale. Les femmes, au contraire, qui, rappelons-le, se montrent plus favorables que les hommes au principe du travail à domicile, lorsqu'elles souhaitent travailler ou continuer à travailler, rejettent, dans la pratique, cette solution lorsqu'on leur demande si elles accepteraient effectivement ce genre de travail pour elles-mêmes $(95 \%)$ ou pour leurs filles $(100 \%)$. Ne faut-il pas voir là le sentiment, plus ou moins conscient, que, malgré ses avantages, le travail à domicile, qui signifie claustration, absence d'indépendance économique et de scolarisation (celle-ci n'étant, en tout cas, pas nécessaire) ne peut être l'instrument de l'évolution de la femme, et que, dans l'avenir : celle-ci devra briser ce cadre traditionnel pour réaliser ses aspirations ?

Cette première approche du problème nous permet de dégager quelques indications générales.

En premier lieu, le groupe des ouvrières se différencie nettement des autres strates de l'échantillon féminin. Au contraire, les hommes et les femmes appartenant aux mêmes milieux sociaux adoptent des positions similaires, à cette réserve près - et combien importante que les femmes ont des points de vue plus évolutifs que les hommes.

En second lieu, un accord unanime se réalise autour d'un petit nombre de professions, qui paraissent concilier les aspirations nouvelles et les exigences de la tradition : carrières médico-sociales et enseignement. À propos des autres métiers, divers critères de choix entrent en conflit. Ils font ressortir l'importance de la motivation économique, d'une part, et indiquent, d'autre part, la permanence des interdits et des modèles traditionnels, sous l'aspect de la crainte du déshonneur et de la promiscuité.

Ces deux derniers points viendront en tête de chacune des parties qui vont suivre, l'une regroupant les motifs d'opposition au travail de la femme, l'autre essayant de dégager les motivations positives de ce même travail. 


\section{Critiques, rejets et restrictions}

\section{a) La promiscuité}

$\underline{\text { Retour à la table des matières du 1er article sur les Marocaines }}$

Une des critiques les plus fréquemment exprimées à l'encontre de certains métiers a trait aux dangers que font courir à la femme des milieux de travail où « elle peut s'amuser avec n'importe qui ». Les risques impliqués par la promiscuité revêtent une telle importance que nous allons les étudier à part, en nous référant à une question qui abordait directement le problème : «Est-ce qu'une femme peut travailler avec des hommes : N'importe où ? jamais ? Dans certains postes de travail seulement?»

Une très faible minorité (uniquement les ouvrières et quelques hommes) opte pour la ségrégation totale des sexes. Quand elles ne choisissent pas cette proposition, les femmes estiment qu'elles peuvent travailler n'importe où avec des hommes (49\%). Un moins grand nombre d'entre elles donnent leur préférence à une solution plus nuancée : «Dans certains postes seulement». Il en va tout autrement avec les hommes, qui, dans leur grande majorité, n'acceptent la promiscuité que sous certaines conditions $(71 \%)(18 \%$ choisissent « N'importe où »). La promiscuité leur apparaît condamnable, surtout lorsqu'elle est assortie de publicité. La secrétaire, la fonctionnaire dans son bureau, l'infirmière à l'hôpital se trouvent en relation avec un petit nombre d'hommes, tous connus, des collègues appartenant généralement au même milieu social. Et surtout, cette promiscuité n'est pas étalée au grand jour. Nul, dans le voisinage, ne peut savoir si la femme a effectivement des collègues masculins. En revanche, la vendeuse, la serveuse de restaurant se trouvent en contact avec un public non choisi, et tout le monde peut en être témoin.

Or l'opinion publique, dont chacun redoute le verdict, exerce sa censure contre les femmes qui travaillent. "Elles s'amusent avec les hommes », entend-on dire un peu partout, et $35 \%$ des hommes sont convaincus du bien-fondé de ce reproche. Des femmes, $50 \%$ reconnaissent l'avoir entendu formuler (mais 17\% seulement le reprennent à leur propre compte, surtout les femmes restant au foyer). Quant à ceux ou celles qui récusent cet argument, ils sont suffisamment pénétrés de son importance pour éprouver immanquablement le besoin de s'en justifier : "Pour un homme qui a confiance en sa femme, peu importe l'endroit où elle travaille ». " De toute façon, si la femme veut faire le mal, elle trouvera toujours le moyen de le faire. »

Pourquoi prône-t-on les professions médicales et celles qui relèvent de l'enseignement ? En grande partie, parce que, dans ces professions, la femme n'est en contact qu'avec d'autres femmes ou avec des enfants, nous répond un groupe de jeunes instituteurs ; ils reconnaissent manquer de confiance à l'égard de la femme, qui, à leur avis, doit être protégée contre ses propres faiblesses. Un autre groupe de jeunes 
adjoints sanitaires donne son accord restrictif à des professions aussi différentes qu'employée de bureau et ouvrière d'usine, à condition qu'elles s'exercent en milieu purement féminin. L'important n'est pas que les femmes accomplissent telle ou telle tâche, mais « qu'elles ne puissent Pas aller rôder avec les hommes ».

Les témoignages abondent dans ce sens. On nous a cité, à diverses reprises, l'exemple de familles bourgeoises où la femme cultivée pourrait prétendre à des emplois intéressants et utiles au pays. Le mari s'y oppose, de crainte que la femme ne soit placée sous l'autorité d'un homme autre que lui. En revanche, il va jusqu'à l'encourager à avoir une vie sociale très active, au sein d'organisations charitables, qui sont animées par des femmes (et à titre bénévole, ce qui supprime toute possibilité d'indépendance économique - autre problème litigieux).

Une jeune fonctionnaire nous dit la difficulté qu'ont la plupart de ses collègues à se faire accepter dans leur travail. On croit difficilement qu'elles puissent s'intéresser réellement à leur tâche. On leur prête des mobiles intéressés : elles ne peuvent être guidées que par le désir de se marier ou de chercher des aventures. Dans une autre question, proposant également une liste de métiers, il est symptomatique de constater que la profession de pilote d'avion est jugée mieux convenir aux femmes que celle de chauffeur de taxi : ces deux professions sont comparables, dans la mesure où elles font toutes deux appel au «sens de la mécanique », généralement dénié aux femmes, mais bien différentes par la résistance nerveuse et les connaissances requises; comme, d'autre Part, de nombreuses Marocaines conduisent déjà leur propre voiture, il semblerait plus facile d'admettre qu'une femme soit chauffeur de taxi; mais ce dernier métier exige, par définition, que l'on ait affaire à des clients pris au hasard - et le danger de promiscuité qu'il implique pèse plus lourd que les exceptionnelles aptitudes réclamées par l'autre.

Pourquoi ce manque de confiance persistant envers la femme ? Pourquoi va-t-il souvent de pair avec des prises de position révolutionnaires, en rupture complète avec la tradition, dans d'autres domaines ? S'agit-il d'une défense de privilèges où l'égoïsme masculin trouve son compte ? On ne peut nier le rôle important joué par cet argument. Mais pourquoi alors tant de jeunes Marocains épousent-ils des Européennes et prônent-ils la femme européenne comme modèle ? Ce qui distingue, en effet, l'Européenne de la Marocaine, c'est précisément sa liberté de mouvement et de contacts avec le monde masculin.

À plusieurs reprises, on nous a fourni l'explication suivante : en Europe, les gens s'épousent par amour. Ici, c'est encore la règle de la contrainte familiale. On n'a aucune raison de faire confiance à une femme dont on sait qu'elle vous a épousé par obéissance à ses parents, ou parce qu'elle ne pouvait rien faire d'autre. Il semble bien, en effet, que les règles traditionnelles du mariage pèsent d'un poids déterminant, non seulement dans le type de relations qu'elles instituent entre les époux, mais encore dans tous les rapports sociaux. Ces règles sont d'ailleurs fortement remises en question par les jeunes générations et leur réforme vient au premier rang des transformations souhaitées.

Quand il s'agit de l'avenir qui se prépare à l'école, la ségrégation a peu de partisans, aussi bien parmi les hommes que parmi les femmes. On lui préfère la mixité complète ou limitée (dans ce cas, jusqu'à la fin du cycle primaire) : "C'est bien, car cela prépare à connaître l'homme, son évolution, ses faiblesses »; «Si la fille est vertueuse, peu lui importe qu'elle soit ou non au milieu de garçons ». 
Ce régime d'enseignement mixte est d'ailleurs le plus courant dans le Maroc actuel, tant dans les établissements primaires du bled que dans certains lycées où les effectifs féminins ont jusqu'alors été trop faibles pour justifier l'ouverture d'établissements réservés aux filles. Cela ne va pas sans difficulté. Les parents du bled en sont parfois mécontents : ils retirent les filles de l'école dès la puberté ; dès que les effectifs le permettent, on crée des écoles séparées. Mais, c'est un fait. Pour le moment, toute une génération est élevée dans ce compagnonnage et, d'après ce qu'indique notre sondage, sans susciter d'opposition profonde de la part des citadins.

Le problème de la Promiscuité a été traité à part, car c'est lui qui est invoqué le plus fréquemment pour justifier les opinions émises.

Mais il existe d'autres motifs d'opposition au travail professionnel de la femme. Une liste de critiques, laissant place aux réponses spontanées, étaient proposées aux sujets interviewés, qui devaient indiquer s'ils les entendaient fréquemment formuler autour d'eux et dans quelle mesure ils les jugeaient eux-mêmes valables.

Les critiques, spontanées ou proposées par la liste, exprimées à l'encontre des femmes qui travaillent peuvent être classées sous trois rubriques.

On redoute que l'intrusion des femmes ne perturbe soit le marché du travail, soit la vie de l'entreprise. Ces arguments d'ordre économique sont les moins nombreux. On critique surtout les répercussions du travail sur la vie familiale et sur la vie personnelle de la femme, d'un point de vue pratique - «Elle néglige son foyer » - et d'un point de vue relatif à la morale sociale traditionnelle - «Elle sort de son rôle ».

\section{b) Perturbations dans le monde du travail}

$\underline{\text { Retour à la table des matières du 1er article sur les Marocaines }}$

Parmi les propositions citées, certaines avaient trait aux relations de travail. Que les femmes soient une cause de disputes dans la vie de l'entreprise, c'est un fait qui est reconnu par de nombreux hommes, mais qui ne l'est pas par les femmes. Que celles-ci prennent la place des hommes, les uns comme les autres le ressentent. Nous verrons plus loin que cette critique peut avoir une acception autre qu'économique. Mais on la retrouve aussi clairement formulée de cette façon. «Si son mari travaille, elle prend la place d'un autre homme en travaillant à son tour ». La femme est accusée de vouloir prendre le rôle de l'homme, mais en trichant, car ce ne serait pas pour gagner un salaire vital qu'elle irait au travail, mais « pour se balader » ou « pour avoir de l'argent à elle ». Cette critique est surtout exprimée par des hommes qui veulent voir, dans le travail de la femme, un simple prétexte pour s'affranchir de son rôle traditionnel. Elle est reprise par les ouvrières, d'une manière plus nuancée : « Si la femme travaille pour gagner son pain et celui de ses enfants, on ne peut rien lui dire ; mais, si c'est pour sortir, on peut lui faire des reproches ». Il faut se rappeler que le Maroc souffre de chômage endémique et, dans ces conditions, il peut paraître scandaleux que plusieurs salaires entrent dans le même foyer, alors que de nombreux chefs de famille cherchent désespérément un emploi. C'est, en tout cas, le point de vue des ouvrières, 
dont la plupart, nous le verrons par la suite, sont elles-mêmes chefs de foyer ${ }^{1}$. Parmi les fonctionnaires et les cadres moyens, le principe du double salaire paraît accepté; en tout cas, dans ce milieu, les femmes qui travaillent sont mariées à des hommes ayant un emploi, eux aussi. Peut-être un autre facteur joue-t-il en la circonstance. Alors que les ouvrières occupent des postes de manœuvre non qualifié, que n'importe quel homme pourrait tenir, les infirmières, les institutrices, les monitrices d'éducation féminine remplissent des fonctions où les hommes pourraient difficilement les remplacer.

\section{c) Manque d'aptitudes et de formation}

$\underline{\text { Retour à la table des matières du 1er article sur les Marocaines }}$

Il faut souligner qu'on dénie très rarement aux femmes les aptitudes nécessaires pour remplir certaines tâches - qu'il s'agisse de qualités physiques ou de dispositions intellectuelles. Cela relève-t-il d'un sentiment foncier d'égalité entre les sexes, qui, par-delà les différences tenant à une phase historique, recèleraient des qualités, des virtualités équivalentes ? En fait, les obstacles dressés par la morale traditionnelle à l'émancipation de la femme, l'inégalité des droits reconnus et la différenciation des rôles sont d'une évidence si aveuglante qu'il n'est point besoin de faire appel à des explications vaguement physiologiques. On peut, cependant, imaginer que, dans la perspective de l'évolution actuelle, qui reconnaît de plus en plus de droits et de liberté à la femme, les objections qui se veulent scientistes, aujourd'hui reléguées dans la pénombre, feront un jour leur apparition.

Si l'on accorde peu d'importance au problème des aptitudes, en revanche les femmes - et les femmes seules - rappellent que le manque de formation constitue actuellement pour elles un obstacle qui les empêche d'accéder à certains métiers. Si la femme recevait une instruction suffisante, elle pourrait, à leur avis, être pilote, contremaîtresse, commerçante, conseillère municipale et même caïd. On pourrait lui attribuer des responsabilités syndicales, si elle était suffisamment instruite : "Ce n'est pas une question de confiance, mais de savoir-faire », "C'est bien, pour une femme instruite, de travailler, car alors elle peut faire un beau métier ». Si la femme se résigne à accepter des tâches qui ont un relent de déshonneur, c'est uniquement parce qu'elle n'est pas suffisamment instruite pour faire autre chose.

Ces réactions sont, avant tout, le fait des ouvrières, toutes analphabètes, qui ressentent vivement l'absence d'instruction comme un obstacle majeur, au-delà duquel se situent toutes leurs chances d'évolution. Il est intéressant de souligner que les ouvrières qui, d'autre part, font souvent preuve du conformisme le plus traditionaliste, consacrant l'homme dans sa supériorité intangible, perçoivent, malgré tout, qu'il existe des moyens de sortir de leur condition de mineures. Et c'est sur ces moyens ou plutôt sur ce moyen essentiel qu'est l'instruction - qu'elles reviennent constamment, traduisant ainsi un désir profond (peut-être obscur pour elles-mêmes) d'aboutir

Voir pp. 115 et 116 de l'édition papier de la revue. Dans cette édition numérique, voir la section $\S 4 \mathrm{a}$ de cet article. 
réellement à un changement, tout en négligeant de mettre en accord cette tendance avec leur position de principe conservatrice. Au contraire, les hommes se montrent souvent plus libéraux sur les questions de principe, mais se désintéressent des moyens pratiques d'aboutir.

L'impérieuse nécessité d'accélérer la scolarisation féminine a été proclamée par les autorités marocaines. La famille royale a montré l'exemple et, dès avant l'indépendance, en présidant souvent à la création d'écoles ou d'œuvres éducatives, le roi et les princesses ont indiqué l'intérêt primordial qu'ils attachaient à de telles réalisations.

Les partis politiques, eux aussi, reprennent ce thème : le droit de la femme à l'instruction figure dans leurs programmes (voir les résolutions du congrès de l'Istiqlal du 2 novembre 1955 [15, no 4, avril 19561, et celles du congrès de l'Union des femmes arabes de l'été 1957 17, $\mathrm{n}^{\circ}$ 291, 20 nov. 1957]. Les sections féminines des différents partis souhaitent consacrer au moins autant d'efforts à l'alphabétisation et à l'éducation domestique de leurs adhérentes qu'à leur formation politique.

La population marocaine a répondu avec faveur à ces appels, depuis quelques années, depuis l'indépendance surtout, comme en témoignent les statistiques scolaires ${ }^{1}$. On peut constater, en outre, des changements dans les attitudes à l'égard de l'enseignement féminin. Pour persuader les parents de laisser instruire leurs filles, il fallait, dans les premiers temps, aménager des programmes spéciaux, consacrés presque exclusivement aux travaux traditionnels de broderie et à l'éducation ménagère. L'enseignement général devait être donné presque à la dérobée [9]. Des diplômes spéciaux consacraient la disparité - et l'infériorité - de l'enseignement donné aux filles (certificat d'études primaires musulmanes des fillettes, par exemple). On entendait dire : «Il faut préparer les filles à leur vocation d'épouse et de mère, non pas à une vie professionnelle », « Il faut que les programmes scolaires soient orientés vers la préparation aux tâches ménagères » [ 10 chap. IV].

Depuis ces temps héroïques, les points de vue se sont complètement modifiés; les parents portent un intérêt actif aux études de leurs filles, les futurs fiancés changent d'attitude : «La famille du fiancé accepte souvent d'attendre un ou deux ans que la jeune fille ait poussé plus avant ses études, au lieu de l'empêcher de sortir » $(9$, p. 16). Un instituteur du bled nous citait le cas d'une de ses élèves, dont le père, lorsqu'elle avait été reçue au certificat d'études, avait exigé - et obtenu - du fiancé un sedaq ${ }^{2}$ plus élevé. Mais, surtout, on n'envisage plus que l'enseignement donné aux filles soit un enseignement au rabais : «La broderie, la couture, ce n'est pas l'école », avons-nous entendu dire bien souvent. Les jeunes filles font maintenant leurs études dans la perspective de se préparer à une vie professionnelle, quitte à l'abandonner une fois mariée. A la question que nous avons posée - « Est-ce que l'enseignement donné aux filles devrait être le même que celui qui est donné aux garçons ? »- seuls $23 \%$ des hommes et $14 \%$ des femmes ont répondu par la négative : la plupart considèrent que la jeune fille doit recevoir, en plus, une formation ménagère - mais cela ne la dispense pas d'acquérir une solide formation générale, «car elle a les mêmes devoirs que l'homme ».

1 Voir p. 94 de l'édition papier de la revue. Dans la présente édition numérique, voir l'introduction de cet article, du $12^{\mathrm{e}}$ au $15^{\mathrm{e}}$ paragraphes.

2 Dot maritale. 


\section{d) Répercussions familiales}

$\underline{\text { Retour à la table des matières du 1er article sur les Marocaines }}$

Plus fréquentes que celles qui viennent d'être examinées sont les critiques qui font état des répercussions fâcheuses du travail professionnel de la femme sur la vie familiale.

Foyer négligé. «Elle néglige son foyer». Cette considération d'ordre pratique vient au premier rang des critiques retenues par les hommes (64\% l'ont entendue, et $35 \%$ la tiennent pour exacte). Elle est reprise par les femmes, mais avec une moindre fréquence (et davantage par les femmes mariées et par celles qui restent au foyer).

La fatigue et la nervosité de la femme qui travaille affectent son comportement à l'égard de ses enfants (les hommes y insistent), ou de son mari (ce sont surtout les femmes qui soulignent ce point). On évoque aussi les perturbations de la vie du couple. Les contacts avec le monde du travail mettent la fidélité de la femme dangereusement à l'épreuve : «Son travail lui permet de connaître d'autres hommes qui sont mieux que son mari : ça l'incite à changer de mari. » Quelques isolés perçoivent cependant des changements bénéfiques : «Comme elle voit moins souvent les membres de sa famille, elle se montre plus gentille », «Elle peut leur acheter tout ce dont ils ont besoin »; et puis, elle acquiert dans son métier des notions utiles pour ses enfants et, surtout, elle peut mieux comprendre la nécessité, pour eux, d'être instruits (opinion surtout masculine). Ces attitudes positives sont celles d'une notable proportion de femmes sans profession $(30 \%)$, d'une petite minorité de femmes fonctionnaires et d'hommes (entre 10 et $15 \%$ ); mais aucune ouvrière ne les adopte.

L'autorité et l'indépendance économique. Les hommes redoutent que la femme n'acquière une autorité nouvelle, qui bouleverse les rapports traditionnels (30\%). Cette préoccupation se retrouve chez les femmes, mais d'une façon moins dominante (17\%). L'indépendance économique que le salaire apporte à la femme détermine, en grande partie, ce changement d'attitude : "Comme elle n'a plus besoin de demander de l'argent à son mari, elle ne le respecte plus »; «Elle n'accepte plus qu'il lui donne des ordres »; «Elle veut être plus libre, elle devient plus exigeante »; « Certaines ne veulent plus s'embarrasser d'un mari chômeur et préfèrent être libres ».

Tous ces reproches se résument dans la formule : «Elles prennent la place des hommes »- critique ambivalente, dont nous avons déjà vu la résonance économique, mais qui, le plus souvent, est significative d'autres préoccupations. "Ce n'est pas le rôle de la femme de travailler » et «C'est un déshonneur pour l'homme de faire travailler sa femme».

Dans le droit coranique, en effet, la femme n'est pas tenue de contribuer aux dépenses du ménage, quelle que soit sa propre fortune, et quelle que soit l'indigence du mari. Ce dernier lui doit l'entretien (nafaça) et, s'il ne satisfait pas à cette obligation 
légale, l'épouse est en droit de demander la dissolution du mariage (c'est un des seuls cas où la femme peut prendre l'initiative du divorce) ou de réclamer l'exécution forcée de son entretien sur les biens du mari. Notons, toutefois, que cette obligation maritale cesse, si la femme se réfugie chez ses parents, si elle entreprend un voyage seule, si elle travaille dehors toute la journée [12, pp. 327-343]

Obligé par la loi, ainsi que par la morale et par l'opinion publique - toutes choses indissolubles en Islam - l'homme digne de ce nom doit donc subvenir seul aux besoins de la famille. En revanche, il est le seul maître des dépenses, c'est lui qui gère le budget et fait les achats ${ }^{1}$. Dans le cas de la famille large, dont il existe encore de fréquents exemples, même dans les villes, ce privilège appartient à l'ancien. « Les fils mariés, même pères de famille, lorsqu'ils vivent sous le même toit, remettent leur argent au père, qui décide lui-même l'achat d'une robe pour sa belle-fille ou d'une chemise pour ses petits-enfants ${ }^{2}$. »Ce sont là des coutumes liées à la famille patriarcale, qui est une unité économique, fortement hiérarchisée, où « pas plus que les jeunes ne peuvent s'émanciper par un travail indépendant », " la femme, accaparée par son rôle biologique, ne peut trouver son indépendance économique par un travail dans le salariat » [19, p. 1756].

Une petite remarque, d'ordre linguistique, nous paraît symptomatique des attitudes que nous avons essayé de décrire. Nous avons rarement entendu parler de la femme comme sujet libre, "autodéterminé », des actions qu'elle engage en travaillant. Un homme ne dit pas «Il est bon ou mauvais qu'une femme travaille », mais «Il est bon ou mauvais de faire travailler sa femme ».

\section{e) Bouleversement des rôles sociaux}

$\underline{\text { Retour à la table des matières du 1er article sur les Marocaines }}$

Domestique, porteuse d'eau, vendeuse, artiste, serveuse, «c'est un déshonneur ». Ambassadeur, ministre, caïd, "jamais ». Aux deux pôles de l'échelle sociale, on retrouve la même opposition, résumée en une formule lapidaire, qui sonne comme un verdict sans appel.

Les fonctions représentatives ou d'autorité sont rejetées par une très forte majorité des sujets interrogés (ambassadeur et ministre, $70 \%$ de rejets ; caïd, 93\%) ; «Elles sont faites pour les hommes »; «Avec les femmes, rien ne marcherait »; «Les Marocains n'aiment pas être commandés par des femmes ». Il ne s'agit, pour certains, que d'une incapacité temporaire, due au contexte social, dont tout le monde se rend compte qu'il évolue à grands pas : «Si les gens l'écoutaient, une femme pourrait être caïd »; «Pas encore, mais oui, dans l'avenir ». Mais, pour beaucoup d'autres, cette attitude est définitive et repose sur des notions religieuses, intangibles, comme

Nous verrons plus loin qu'il y a une forte tendance à partager les responsabilités dans ce domaine. Mais ici, nous essayons de définir les positions traditionnelles, qui sont encore assumées telles quelles par beaucoup de Marocains, et qui permettent d'expliquer nombre de réactions, même chez ceux qui se veulent modernistes.

2 Documents de FIRAM sur le douar Oujda de Salé, p. 16. 
l'explique très bien le décret d'Al Ahzar auquel nous avons déjà fait allusion ${ }^{1}$. Se référant à un hadith - " jamais le peuple qui confie ses affaires à une femme ne connaîtra le succès » - et à un verset du Coran (IV, 34) - «Les hommes décident pour les femmes, étant donné que Dieu a accordé sa préférence aux uns sur les autres »les oulémas considèrent que la loi religieuse confie l'autorité aux hommes et interdit à la femme l'accès des fonctions publiques.

Tous les «docteurs de la loi » n'approuvent cependant pas cette interprétation rigoriste des textes sacrés. Dès la fin du XIXe siècle, des juristes, des théologiens arabes ont été les plus fermes soutiens du mouvement d'émancipation féminine [5 ${ }^{2}$ ], dont ils pouvaient retrouver les pionnières dans les siècles d'or de l'islam. Le MoyenOrient compte maintenant des femmes médecins, diplomates, ingénieurs, professeurs. En Irak, pour le premier anniversaire de la révolution (14 juillet 1959), une femme a été nommée ministre des municipalités 15, p. 166]. Et ce n'est pas seulement en tant que roi du Maroc, mais en tant qu'iman des croyants, que S. M. Mohammed V a prêché pour l'évolution des femmes de son pays. "Victime d'une interprétation erronée de notre sainte religion, victime de ce qu'on peut nommer les fausses traditions, longtemps tenue à l'écart, la femme a saisi toutes les occasions pour prendre une part active à la vie du pays, s'intéresser aux choses publiques, aux activités de l'esprit et faire ses preuves dans divers domaines » [18, p. 152].

Ce bilan, aussi complet que possible, des critiques et des restrictions opposées au travail de la femme ne doit pas dissimuler le fait qu'elles ne sont pas unanimement approuvées. Un pourcentage non négligeable des sujets interrogés leur dénie toute valeur: $10 \%$, des hommes ne reprennent aucune de ces critiques à leur propre compte, bien que tous reconnaissent en avoir entendu formuler autour d'eux. Certains, d'ailleurs, restreignent la portée de cet opprobre social à quelques groupes jugés retardataires : "Ce sont les vieux qui disent ça »; «Dans le milieu ouvrier, on ne critique pas les femmes qui travaillent». Nous avons pu constater que, dans l'ensemble, la fréquence des attitudes négatives chez les hommes est notablement plus élevée que chez les femmes. Près de la moitié de celles-ci rejettent toute critique : «Il vaut mieux travailler que de se priver, soi et ses enfants » ou «que de traîner »; «C'est par jalousie qu'on fait des reproches », «parce que tout le monde voudrait bien travailler ».

Comme nous l'avons déjà noté plus haut, les questions directes engendrent des réactions de défense, qui amènent les intéressées, personnellement visées par ces reproches - en particulier les ouvrières - à dépasser les points de vue de la morale traditionnelle, à laquelle elles se montrent attachées à propos de beaucoup d'autres questions moins directes.

Quant aux femmes sans profession, elles se montrent beaucoup plus critiques que la moyenne du groupe féminin à l'égard des femmes qui travaillent, avec toute la fierté de bonnes ménagères qui, elles, ne négligent pas leur intérieur, et de femmes sérieuses qui, elles, ne sortent pas. C'est l'occasion - passagère - de valoriser, à leurs propres yeux, le genre de vie qu'elles mènent et dont elles mesurent, d'autre part, les imperfections, puisqu'une grande partie d'entre elles souhaiteraient travailler.

\footnotetext{
1 Voir p. 100 de l'édition papier de ce numéro de la revue. Dans l'édition numérique de cet article, voir la section $\$ 2$ [choix des métiers].

2 Voir le chapitre intitulé «Intercession de la femme ».
} 
Même si les femmes ont parfois des positions de principe en retrait sur celles des hommes, elles perçoivent manifestement que leur condition est en pleine transformation et elles adhèrent à cette évolution. C'est ce qui ressort de l'intérêt qu'elles portent aux moyens pratiques de modifier leur sort - à l'instruction en particulier. Les hommes, à un moindre degré, prennent, eux aussi, conscience de cette évolution, comme en témoignent leurs opinions sur la mixité scolaire.

Mixité ou ségrégation des sexes : problème litigieux entre tous et pierre de touche de la transformation des mentalités, puisque les principales restrictions et critiques tournent autour de la promiscuité dans le travail. Mais aussi, problème interdépendant d'autres problèmes, dont les solutions sont nécessairement liées à l'évolution de la structure familiale et des règles du mariage ; problème qui dénote un état de malaise, né du conflit entre les modèles et les situations vécues, entre les modèles traditionnels et les aspirations nouvelles - conflit qu'on tranche le plus souvent par des dérobades. Dans des situations entièrement nouvelles, qui répondent aux besoins d'une économie et d'une société modernes, on continue, dans l'ensemble, à juger en fonction des règles de la morale traditionnelle.

Le temps ne serait-il pas venu de tracer une voie nouvelle ? C'est a quoi s'efforcent un certain nombre de penseurs et de dirigeants qui, sans rien renier de leur héritage, tentent d'établir un pont entre le passé et l'avenir, en retournant aux sources de la religion, invoquée par d'autres comme la justification de l'immobilisme, ou bien en cherchant, dans les ébranlements de l'évolution moderne, à frayer de nouvelles voies.

\section{Motivations}

$\underline{\text { Retour à la table des matières du 1er article sur les Marocaines }}$

Il est déjà apparu à plusieurs reprises que les préoccupations économiques interviennent largement pour modifier les positions de principe à l'égard du travail de la femme. Une question nous permet d'aborder plus directement ce problème : « À votre avis, quelles sont les principales raisons qui poussent les femmes à travailler ?». (On sollicitait une réponse spontanée, puis on soumettait une liste de réponses proposées.)

\section{a) Les besoins économiques familiaux}

Les réponses spontanées, aussi bien que les réponses choisies dans la liste proposée, font ressortir la prépondérance d'un facteur : les besoins économiques familiaux (réponses spontanées : 74\% chez les femmes, 64\% chez les hommes; liste : $82 \%$ chez les femmes, $78 \%$ chez les hommes). Si la femme travaille, c'est «par besoin », «à cause de la misère », " parce que son mari ne gagne pas assez ou parce qu'il ne 
travaille pas », « pour se vêtir, se nourrir et acheter du savon »; « certaines, c'est le mari qui les pousse à travailler ; celles qui sont seules, c'est pour manger ».

Cette nécessité économique est ressentie au niveau des besoins élémentaires de la famille : se nourrir, se loger. Les besoins moins impératifs sont rarement évoqués (14\% chez les hommes et les femmes) : «pour acheter des bijoux », « pour suivre la mode », «pour s'acheter de beaux meubles ». Le salaire féminin joue rarement un rôle d'appoint permettant à la famille de vivre dans une plus grande aisance, ou à la femme de satisfaire quelques fantaisies : c'est un salaire vital, au sens propre; comme nous l'avons déjà noté, cette attitude est surtout le fait du milieu ouvrier, où la femme qui travaille est, le plus souvent, la seule à apporter un salaire dans une famille plus ou moins nombreuse.

Voici, à titre d'exemple - combien significatif quelles sont les ressources des 12 ouvrières interrogées. (Rappelons que les 12 ouvrières d'usine ont été choisies au hasard dans un établissement qui en emploie 112.) Il y a 9 foyers qui vivent avec le seul salaire de la femme (dans les 3 autres foyers, d'autres personnes contribuent également aux dépenses du ménage) : 2 femmes (30 et 43 ans) vivent seules ; 2 (16 ans et 29 ans) ont une personne à charge (l'une sa mère, l'autre sa sœur) ; 2 (30 ans et 35 ans) ont deux personnes à charge (l'une un fils et son mari chômeur depuis trois ans ; l'autre une petite fille et son mari, chômeur partiel) ; 1 (40 ans) a trois personnes à charge (une petite-fille, sa mère et son mari, grand infirme) ; 1 (18 ans) a quatre personnes à charge (mère, frère et sœur en bas âge, beau-père chômeur) ; 1 (23 ans) a six personnes à charge (ses deux enfants, une sœur, sa mère, son père et un frère chômeurs).

Ces chiffres, qui peuvent paraître excessifs, sont cependant confirmes par d'autres enquêtes, notamment celle qui a été menée, dans les bidonvilles de Casablanca, par A.-M. Baron et H. Pirot. A propos de la situation des femmes mariées qui travaillent, ceux-ci constatent que, dans $50 \%$ des cas, le mari ne travaille pas. Il est vieux, malade, chômeur temporaire ou permanent. Soulignons que ce pourcentage ne concerne que les femmes mariées ; on obtiendrait, évidemment, un pourcentage nettement plus élevé et coïncidant probablement avec ceux de notre échantillon si l'on prenait en considération les femmes veuves ou divorcées qui sont chefs de famille. "Cette notion de femme seule, chef de foyer, est, en effet, une caractéristique du prolétariat. Quelles en sont les causes ? Tout d'abord, l'émiettement plus grand de la famille : la femme divorcée ne trouve plus toujours refuge au sein du groupe familial. C'est ensuite, dans une certaine mesure, la carence de l'homme et la nécessité, pour la femme, de gagner la vie de sa famille. La proportion des femmes seules, chefs de foyer, est d'autant plus grande qu'on descend dans les différentes couches du prolétariat... et atteint le chiffre de $42 \%$ (contre $58 \%$ d'hommes chefs de foyer) dans les foyers très pauvres. » $\left[4\right.$, p. $43 ; 8$, p. $\left.153^{1}\right]$

La satisfaction des besoins économiques de la famille est reconnue, par tous les sujets interrogés, comme un facteur déterminant. Rappelons ici ce que nous avons dit à propos du choix des métiers : beaucoup d'entre eux ne sont acceptés que par nécessité, parce qu'on a besoin d'argent et qu'on ne dispose d'aucune autre possibilité de gagner sa vie. De même, la nécessité économique infléchit les positions à l'égard du travail à domicile, auquel vont les préférences de principe, mais que, dans la

1 Dans les bidonvilles de Port-Lyautey, sur environ 900 femmes qui travaillent, 134 sont chefs de famille. 
pratique, on rejette, à cause de sa médiocre rentabilité. De même, elle domine les attitudes prises en fonction des diverses situations familiales. Tout le monde est pour le travail de la femme seule $(100 \%)$ avec quelques réserves si elle a des enfants, et une majorité s'élève contre celui de la femme âgée, qui n'est accepté que par $42 \%$ des hommes et $32 \%$ des femmes. Le travail de la femme mariée sans enfant et celui de la jeune fille recueillent exactement le même pourcentage d'approbations ( $75 \%$ chez les hommes; $85 \%$ chez les femmes). Les oppositions au travail de la femme mariée avec enfant sont un peu plus nombreuses, surtout chez les hommes, qui ne l'approuvent que dans $60 \%$ des cas (femmes : $75 \%$ ).

Cette énumération appelle quelques commentaires. On peut affirmer, sans grand risque d'erreur, que l'unanimité en faveur du travail de la femme seule constitue l'un des signes de la dislocation des structures familiales traditionnelles. On considère que la femme seule doit subvenir à ses propres besoins et à ceux de ses enfants. Il ne paraît plus normal qu'elle soit prise en charge par sa propre famille, en attendant un remariage, alors que, traditionnellement, la veuve ou la divorcée était sûre d'être accueillie au foyer de son père, de son frère ou, aussi bien, d'un oncle ou d'un cousin. C'était là un devoir familial indiscutable qui, aux yeux de nombreux juristes, était la justification de la dot versée par le mari au moment du mariage [6, pp.110-119].

Autre fait significatif : il y a une égale opposition au travail de la jeune fille et à celui de la femme mariée sans enfant. Pourquoi ? Dans l'ensemble,nous l'avons dit, le travail de la femme est uniquement conçu en fonction de la nécessité économique et, dans cette perspective, la jeune fille, n'ayant pas de charges familiales, n'a pas besoin d'un salaire. C'est du moins l'optique des ouvrières (65\% en faveur du travail de la jeune fille, avec de fortes restrictions, et $75 \%$, en faveur du travail de la femme mariée sans enfant). À ce propos, il faut souligner une fois de plus la forte divergence des diverses strates de l'échantillon féminin. Les femmes fonctionnaires sont favorables au travail de la jeune fille (100\%). Les femmes sans profession ont la même tendance que les ouvrières et les hommes : plus favorable au travail de la femme mariée qu'à celui de la jeune fille.

Quant à la présence d'enfants au foyer, elle intervient également, de façon contradictoire, suivant les différentes strates. Pour tout l'échantillon, à l'exception des ouvrières, la présence d'enfants diminue la fréquence des choix positifs en faveur du travail de la femme. Cela est particulièrement net chez les fonctionnaires (pour le travail de la femme mariée sans enfant : $90 \%$, dont 25\% assortis de restrictions ; pour le travail de la femme mariée avec enfants : $33 \%$, toujours avec des restrictions). La pression économique étant moins forte, le premier devoir de la femme n'est pas de sortir pour gagner le pain de ses enfants, mais de rester auprès d'eux. C'est d'ailleurs dans ces strates qu'on prend conscience de problèmes non économiques soulevés par le travail de la mère de famille - par exemple, la garde des enfants - alors que cette préoccupation n'apparaît jamais chez les ouvrières. Pour ces dernières, les enfants sont autant d' « excuses » au travail de la femme (100\% de choix positifs, si la femme mariée a des enfants ; $75 \%$ si elle n'en a pas). En ce qui concerne la femme seule, les choix positifs sont de l'ordre de $90 \%$ dans les deux hypothèses, mais les restrictions sont plus nombreuses si l'intéressée n'a pas d'enfant.

Les ouvrières ne peuvent imaginer le travail hors des perspectives d'une écrasante contrainte économique, qui ne laisse place à aucune autre aspiration et qui en compromet probablement l'éveil. Comment pourraient-elles d'ailleurs concevoir leur 
épanouissement dans les conditions de travail qui sont les leurs actuellement, et ne pas souhaiter y échapper, dès qu'elles en ont la possibilité matérielle ?

\section{b) Décalage entre les modèles et les comportements}

\section{$\underline{\text { Retour à la table des matières du 1er article sur les Marocaines }}$}

Mais, dans cette représentation contraignante du travail qui, certes, a des bases réelles, on pourrait peut-être déceler le désir des intéressés d'avoir bonne conscience à l'égard des impératifs de la morale traditionnelle : ne pas prêter le flanc à la critique, en assumant la pleine responsabilité de leur condition, mais se représenter, à soimême et aux autres, comme soumises à une nécessité extérieure dont personne ne peut leur faire grief. En se conformant ainsi aux modèles traditionnels - seule la femme claustrée est honorable - les ouvrières ne peuvent que chercher à se justifier de leur condition, et non pas à en dégager les valeurs positives. Mais, ce faisant, ne compromettent-elles pas leurs chances d'évolution ? A-t-on compris toute l'importance qu'il y aurait à formuler explicitement les valeurs positives que recèle le travail féminin, à leur donner vie en leur donnant un nom, à les répandre avec insistance dans le public, pour faire évoluer les mentalités ?

Il existe, bien sûr, des failles dans ce comportement traditionaliste. Nous avons déjà signalé que ces mêmes femmes reconnaissaient l'importance de l'instruction, qui est un des moyens essentiels d'améliorer leur sort. On assiste même à une prise de conscience plus nette et plus complète dans des groupes restreints, comme le mouvement syndical, où l'on s'efforce de convertir les mentalités traditionalistes et de faire expérimenter aux intéressées leurs forces vives, leurs valeurs propres, soit dans la défense de leurs intérêts individuels, soit dans les manifestations de masse. Le Ier mai, les femmes de ménage, en blouse de travail ou en djellaba, balai en main, participent au défilé, et leur responsable nous déclarait : "C'est un bien pour l'avenir du Maroc et pour les femmes elles-mêmes qu'elles travaillent. Nous espérons qu'il y en aura de plus en plus. »

Mais on doit constater que, dans leur grande majorité, les intéressées se montrent encore incapables de prendre conscience de leur dignité de femme qui travaille. Telle cette jeune ouvrière de 16 ans, à qui son abnégation vaudrait ailleurs tous les prix de vertu : depuis l'âge de 10 ans, c'est elle qui fait vivre sa mère. Mais, loin d'en tirer vanité ou même seulement de prendre conscience de son utilité, elle a honte d'ellemême et dit d'un air malheureux : "je ne suis pas une femme bien, puisque je travaille. » Telles la plupart de ces jeunes femmes, dont le tableau précédent a indiqué les responsabilités familiales, et qui souffrent de ce même sentiment d'infériorité. Chez la plupart d'entre elles, on dénote également la même aspiration à ne plus travailler, si les conditions économiques le leur permettaient. À la question : "Quelle femme souhaiteriez-vous être ?», nombreuses sont celles qui répondent : "Une femme heureuse qui reste chez elle et que son mari nourrit. » En s'enfermant dans les modèles traditionnels, non seulement elles ne discernent pas les valeurs positives de leur condition actuelle, mais, du même coup, elles détournent leur énergie des moyens propres à l'améliorer. 
Il faut reconnaître que, sauf de rares et éclatantes exceptions, la plupart des femmes cultivées appartenant au milieu bourgeois ne font pas profiter leurs concitoyennes de leurs propres acquisitions, mais continuent à leur donner comme modèle celui de la femme consacrée à la vie privée. L'état d'esprit le plus généralement répandu consiste à rechercher les circonstances atténuantes aux infractions que constituent ces conditions de vie nouvelles, plutôt qu'à élaborer un nouveau code, qui aide les femmes à prendre conscience de leur dignité de travailleuses. Celles-ci sont en proie à de perpétuels tiraillements, essayant tant bien que mal d'accorder les impératifs des situations nouvelles avec leur conscience et l'opinion publique.

$\mathrm{Si}$, à juste titre, tous les efforts convergent vers le développement économique du pays, il est évident que cet élan est souvent freiné par des comportements, des mentalités archaïques, qu'il faut convertir pour obtenir l'adhésion active des hommes condition indispensable de l'efficacité matérielle. L'apport original et irremplaçable des femmes à ce mouvement ne peut être obtenu qu'en les aidant à y trouver une place clairement et publiquement définie. Le dynamisme de l'émancipation féminine peut alors être un puissant moteur au service de la nation.

Mais le service du pays est-il actuellement perçu comme une motivation du travail féminin ?

\section{c) La notion d'utilité sociale}

$\underline{\text { Retour à la table des matières du 1er article sur les Marocaines }}$

La notion d'utilité sociale, de service national, atteint la même fréquence que les motivations précédentes chez les hommes $(75 \%)$, les femmes fonctionnaires (95\%) et les femmes qui restent au foyer $(60 \%)$. Elle est inexistante chez les ouvrières.

Il faut noter, cependant, que cette préoccupation est moins immédiatement sensible que les précédentes, car si, lorsqu'elle est suggérée par les listes de choix, les « oui » sont très nombreux, elle ne se manifeste pas au niveau des réponses spontanées (3\% chez les hommes, 6\% chez les femmes fonctionnaires, $0 \%$ chez les ouvrières et les femmes restant au foyer).

Les autorités marocaines ont pourtant fortement insisté sur la nécessité d'associer les femmes au développement du pays, tant pour le bien du Maroc - qui ne peut se priver de l'apport irremplaçable des femmes - que pour les femmes elles-mêmes, qui, en associant leur émancipation à celle de tout un peuple, se prémunissent contre les dangers d'une évolution anarchique, en opposition avec leur milieu. La princesse Lalla Aïcha déclarait : "L'émancipation ne consiste pas surtout à briser une tutelle, une entrave, mais, avant tout, à se libérer en tant qu'individu, à s'affirmer en tant que membre d'une collectivité, titulaire de droits, et appelé à remplir des devoirs... Cette émancipation voulue, consentie par la femme, doit néanmoins être orientée, pour s'insérer dans le cadre des institutions du pays, s'effectuer en harmonie avec ses lois fondamentales, dans le respect de ses saines traditions. » [18, p. 153] 
Faut-il conclure du décalage entre les réponses spontanées et celles qui sont suggérées par les listes proposées que ces notions, sur lesquelles reviennent les autorités - peut-être parce que, justement, elles n'ont pas encore fait leur chemin - sont acceptées, reconnues, mais n'ont pas encore suffisamment pénétré les consciences pour se manifester spontanément et pour gouverner les comportements ? Relevons cette réponse, faite par une femme appartenant elle-même à la haute bourgeoisie, mais qui travaille dans un établissement d'éducation : "Rares sont celles qui pensent à l'intérêt national. Pourtant, une femme instruite, même riche, devrait faire profiter les autres de son instruction et penser au bien du pays »; et la réflexion d'un homme : «Elles ne pensent pas à être utiles à leur pays. Ça a un sens vague. Les femmes de demain, peut-être »; celle d'un militant syndicaliste chevronné : «C'est le fait d'un petit nombre de femmes progressistes. »C'est le même militant qui retient l'utilité nationale comme seul critère dans le choix des métiers : «La femme peut travailler n'importe où, même dans les barrages, si c'est l'intérêt du pays. » D'une façon moins absolue, certains tiennent compte de ce critère pour approuver des professions (qui bénéficient, d'autre part, d'un préjugé favorable), telles que celles d'institutrice, d'infirmière, de fonctionnaire : "De cette façon, la femme sert son pays : c'est un honneur. » Citons encore la discussion qui se déroulait au cours d'un stage d'animateurs ruraux (paysans chargés de responsabilités particulières dans leur village). Un des participants empêchait sa femme (institutrice ou infirmière) d'exercer sa profession ; il s'est trouvé une majorité de paysans pour affirmer qu'on devrait obliger l'homme à laisser travailler sa femme, au nom de l'intérêt de la collectivité villageoise à laquelle elle pourrait rendre grand service.

Mais ces réactions sont isolées. Elles sont le fait d'une petite minorité consciente du dynamisme d'une telle motivation. Il est même assez surprenant que celle-ci ne soit pas plus répandue, quand on considère l'intérêt passionné avec lequel les femmes ont suivi les péripéties qui ont précédé l'accession de leur pays à l'indépendance et la part, directe ou indirecte, qu'elles ont prise dans la lutte; quand on considère également combien ce thème est vivant au Maghreb, en Algérie particulièrement, et en d'autres pays arabes, comme en témoignent de nombreuses professions de foi - entre autres, celle de Leïla Baalbaki, jeune Libanaise qui, prétendant dévoiler le vrai visage de la jeunesse arabe, rejette avec véhémence la famille et la tradition, comme un carcan insupportable aux aspirations des jeunes. Cependant, son individualisme forcené se combine avec un sentiment très fort de la solidarité nationale et de ses devoirs à l'égard de l'État : "L'État est comme la mer, la miche de pain, le bouquet de pensées»; « Nous voulons insuffler l'effervescence de notre sang dans les artères desséchées de l'État »; « Nous ne réussirons jamais à imposer le respect à la grande famille humaine aussi longtemps que... nous n'apporterons pas le concours de nos bras et de nos épaules, afin d'achever l'édification de l'État nouveau » [3, pp. 158$160]$. 


\section{d) L'intérêt personnel de la femme}

$\underline{\text { Retour à la table des matières du 1er article sur les Marocaines }}$

Les autres motivations revêtent beaucoup moins d'importance.

Est-ce que la femme recherche des satisfactions personnelles dans l'exercice d'une profession? Seules les femmes fonctionnaires de la santé et de l'enseignement pensent qu'une femme peut travailler par goût pour une profession $(70 \%$ réponses spontanées : $6 \%)$. C'est une considération étrangère aux ouvrières $(0 \%)$, de peu d'importance pour les femmes qui restent au foyer (20\%; réponses spontanées : $0 \%$ ) et pour les hommes (3\% réponses spontanées : $0 \%$ ).

On envisage rarement que la femme puisse travailler pour échapper à l'ennui ; cette idée est exprimée plus fréquemment par les fonctionnaires $(46 \%)$ que par les femmes restant au foyer (30\%) et par les hommes (32\%) [ouvrières : $0 \%$ Les commentaires sont peu abondants. Retenons celui-ci : «La femme développe son esprit et apprend ce qu'est la vie. »

Pareillement, la recherche de l'indépendance - et, plus particulièrement, de l'indépendance économique - apparaît comme un facteur négligeable. Seules les femmes restant au foyer citent ce motif aussi souvent que les besoins économiques familiaux et les études des enfants (réponses suggérées par la liste proposée : 60\%). Cette attitude traduit les préoccupations de femmes qui peuvent rarement disposer d'argent, puisque, ainsi que nous l'avons déjà indiqué, traditionnellement, la femme ne gère pas le budget familial et n'a donc jamais d'argent entre les mains. Chez les femmes qui travaillent, cette aspiration est beaucoup plus rarement exprimée (ouvrières : 8\% ; fonctionnaires : 20\%). Peut-être ce besoin est-il maintenant satisfait. Peut-être les intéressées ont-elles fait l'expérience que, leur salaire étant entièrement absorbé par les besoins familiaux, elles ne pouvaient, de toute façon, pas en disposer pour ellesmêmes. Peut-être craignent-elles de se faire mal juger en mettant en avant des aspirations qui ne soient pas strictement liées aux besoins de la famille. Chez les hommes, les hommes mariés (55\%), nettement plus que les célibataires $(21 \%)$, font état de cette motivation. Ces derniers, plus jeunes, ont une autre conception du partage des responsabilités familiales et souhaitent, dans leur ensemble, que les ressources soient mises et gérées en commun.

La place des aspirations personnelles est donc médiocre, aussi bien aux yeux des hommes qu'à ceux des intéressées elles-mêmes. Il semble bien que, dans le travail, la femme ne recherche pas tellement des satisfactions, un moyen d'épanouir sa personnalité, et qu'elle n'y voit pas davantage l'arme essentielle de son émancipation. On constate d'ailleurs que, dans les revendications qu'expriment, en particulier, les résolutions d'assemblées féminines, si le droit au travail n'est jamais omis, il n'apparaît jamais non plus au premier plan.

Est-ce seulement pour prévenir les critiques répandues contre le travail féminin critiques qui, rappelons-le, tournent essentiellement autour des répercussions 
fâcheuses de ce travail sur la vie familiale ? Est-ce une réaction provisoire, susceptible d'évoluer rapidement vers d'autres formulations ? Toujours est-il qu'actuellement le travail féminin est conçu, avant tout, dans la perspective du service de la famille et, éventuellement, d'une collectivité plus large : la nation. La femme n'y voit pas une façon de s'affranchir de son rôle traditionnel, mais plutôt le moyen d'assumer ce rôle dans le cadre d'une situation nouvelle. Les modalités sont différentes, car la transformation de la société globale crée des exigences nouvelles, mais les objectifs restent identiques.

L'extension du travail féminin et l'indépendance économique qui en découle aboutiront-elles, en fait, cependant à transformer radicalement le statut de la femme en l'affranchissant de son rôle traditionnel ? Nous ne prétendons pas en débattre ici, mais, restant fidèle à notre propos initial, nous nous sommes limitée à présenter les attitudes qui s'expriment actuellement.

Après avoir passé en revue les différentes attitudes que suscite le travail de la femme, est-il possible d'en tirer des conclusions ? Il va de soi que les « oui » des ouvrières et les «non » des femmes restant au foyer ne peuvent pas s'additionner pour faire une moyenne. Mais il est évident aussi qu'à l'intérieur d'une même strate et chez un même individu on relève des contradictions. Les critiques annulent-elles les motivations ou bien la force de ces dernières réduit-elle les critiques à des oppositions purement verbales, d'autant plus violentes qu'elles interviennent seulement à titre compensatoire ? Les niveaux auxquels se situent critiques et motivations sont bien différents; entre le bien moral, identifié à la norme traditionnelle, et les besoins économiques, une transaction est-elle possible ? On pourrait reprendre encore beaucoup d'exemples d'attitudes qui semblent s'annuler l'une l'autre, entrer en conflit ou être tellement décalées qu'il paraît impossible d'en faire la synthèse. En tout cas, ces difficultés sont significatives du débat profond que soulève, dans le pays et au sein même de chaque individu, ce problème saisi en pleine évolution, au moment où les directions se cherchent, dans le cadre d'une société elle-même en pleine transformation.

Nous allons présenter ce qui nous paraît quand même être une synthèse celle que réalise chaque individu lorsque, tiraillé entre des forces contradictoires, il est quand même obligé de faire un choix personnel. En guise de conclusion, nous allons donc présenter brièvement les réponses obtenues à la question la plus directe : «Et vous, souhaitez-vous travailler? (ou que votre femme travaille ?) ».

Des femmes et des hommes, 60\% répondent positivement. Dans leur ensemble, les hommes se montrent un peu plus favorables, car leurs refus sont souvent assortis de restrictions, alors que, chez les femmes, ce sont, à l'inverse, les choix positifs qui sont restrictifs. Chez les femmes, nous retrouvons la divergence que nous avons relevée tout au long de cette étude - deux tiers des ouvrières préféreraient cesser de travailler, alors que deux tiers des femmes restant au foyer souhaiteraient, au contraire, ne plus rester chez elles ; la proportion des fonctionnaires qui veulent continuer à exercer leur profession est encore plus forte $(80 \%)$.

Compte tenu de tous les arguments positifs, de toutes les critiques et les restrictions, c'est finalement dans un sens favorable au travail féminin que la majorité des sujets interrogés font leur choix, s'engageant ainsi eux-mêmes, avec leur famille, et, par là même, avec tout le pays, sur un chemin irréversible. 


\section{Bibliographie}

$\underline{\text { Retour à la table des matières du 1er article sur le Maroc }}$

1. ADAM, A. «Le bidonville de Ben Msik », contribution à l'étude du prolétariat musulman au Maroc. Annales de l'Institut d'études orientales, t. VIII, 1949-1950. Alger, Faculté des lettres, Éd. Jules Carbonel. 140 p., photos, glossaire. (Tirage à part.)

\section{Annuaire statistique du Maroc.}

3. BAALBAKI, Leïla. « Nous, sans masque, ou la jeunesse arabe dévoilée ». Orient, $\mathrm{n}^{\circ}$ 11, 3e trim. 1959, pp. 145-163. Conférence traduite de l'arabe par M. Barbot.

4. BARON, A. M. ; PIROT, H. « La famille prolétarienne » in : La famille marocaine. Rabat, Les cahiers de faits et idées, 1955, pp. 25-55

5. BERQUE, J. Les Arabes d'hier à demain. Paris, Éditions du Seuil, 1960, 285 p.

6. BOUSSER; KHELLADI. «Enquête sur le trousseau (choura) et la dot (sadaq) au Maroc ». Revue africaine, no 390-391 (1er et 2e trim. 1942), pp. 104-155

7. Documents nord-africains. 6, rue Barye, Paris.

8. ÉTIENNE, J. D'; VILLÈME, L.; DELISLE, St. L'évolution sociale au Maroc. Paris, Éd. Peyronnet, 230 p., ill. (Collection Les cahiers de l'Afrique et de l'Asie.)

9. La femme musulmane aи Maroc (manuscrit anonyme, 1954?) 58 p. bibl.

10. KOUBI, R. Évolution de la femme musulmane au Maroc, 31 mars 1956.Mémoire tiré au duplicateur, $38 \mathrm{p}$.

11. MATHIEU, Dr J.; MANEVILLE, R. « Niveaux de vie et alimentation des prolétaires musulmans de Casablanca ». Manuscrit, février-décembre 1949, 161 p., cartes, photos, glossaire, bibl.

12. MILLIOT, L. "Introduction à l'étude du droit musulman ». Paris, Recueil Sirey 1953, 822 p., bibl., glossaire, table analytique des matières, table alphabétique.

13. MONTAGNE, R. Naissance du prolétariat marocain, enquête collective 1949-1950. Paris, Peyronnet, 290 p., bibl., index des noms propres, nombreux graphiques, cartes. (Collection Cahiers de l'Afrique et de l'Asie.) 
14. MONTEIL, V. L'arabe moderne. Paris, Klincksieck, 1960, 386 p., index des notions et des termes techniques arabes. (Études arabes et islamiques, études et documents, III.)

15. Revue de presse, 46, rue Bencheneb, Alger.

16. TAHIR, El Haddad. « Notre femme dans la loi et dans la société ». Revue des études islamiques, 1935, cahier III, pp. 202-230. Traduction analytique de M. Mutafassig.

17. TILLION, G. L'Algérie en 1957. Paris, Éditions de minuit, 1957, 121 p.

18. TOUMLILINE II. Aspects de l'éducation. Rabat, monastère de Toumliline 1957.

19. VIEILLE-MICHEL, A. «Famille, société industrielle et démocratie ». Esprit, nov. 1960. 
III

Femmes et professions au Maroc

II

\section{"Évolution et travail professionnel de la femme au Maroc"}

Khadidja Nouacer,

Retour à la table des matières du livre

Table des matières du $2^{\mathrm{e}}$ article sur les femmes au Maroc

1. L'opinion publique devant l'accession des femmes à la vie professionnelle

a) L'opinion dans les différents milieux sociaux

b) Prestige attribué aux différentes professions

$\underline{\text { Retour à la table des matières du 2e article sur les Marocaines }}$

L'évolution de la femme au Maroc revêt divers aspects; l'accès au travail professionnel en est un des plus importants, des plus lourds de conséquences. L'opinion publique ne s'y trompe pas, qui réagit vigoureusement - et diversement - devant ce fait nouveau.

Cette évolution ne peut s'éclairer qu'en fonction de l'historique de l'apprentissage et de l'enseignement qu'ont reçus les jeunes filles marocaines. 


\section{Les différentes étapes de l'enseignement et du travail féminins}

$\underline{\text { Retour à la table des matières du 2e article sur les Marocaines }}$

L'éducation de toutes les fillettes marocaines se limitait jadis à l'apprentissage de la couture et de la broderie. À cet effet, les parents confiaient leurs enfants à une maallema qui devait les éduquer et leur apprendre les travaux féminins. Le choix de ces maallema impliquait une certaine discrimination, une hiérarchie établie entre les différentes catégories d'éducatrices.

Les maallema qui faisaient des ouvrages raffinés travaillaient pour la classe bourgeoise. C'est en fonction de leur clientèle et de la qualité des travaux présentés dans les familles que les parents les choisissaient pour leur confier leurs fillettes, à qui elles enseignaient la broderie, la couture, la dentelle.

Ensuite venaient les maallema tapissières ; elles étaient moins appréciées et se voyaient confier les enfants de la classe moyenne ou de la classe populaire. Quant aux parents qui désiraient tout simplement que leurs fillettes gagnent leur vie le plus tôt possible, ils les plaçaient chez des femmes qui faisaient de la confection et qui avaient comme clients les commerçants. Ces femmes étaient moins bien considérées, car elles allaient elles-mêmes chercher leur travail, discutaient avec le client ; ou encore, très souvent, le client leur rendait visite, venait leur apporter le travail à domicile - ce que les voisins voyaient avec peu de sympathie.

L'organisation des écoles et des ateliers d'apprentissage et des ouvroirs a marqué la première étape de l'évolution. À chaque école ou ouvroir était attachée une maallema, et c'est à elle que les parents s'adressaient pour inscrire leurs fillettes dans l'établissement, de préférence à la directrice ou à l'institutrice. Ils demandaient qu'on mette leurs enfants à l'atelier de broderie ou de tapisserie, le plus tôt possible. Il était très difficile de faire admettre, à l'époque, que les fillettes douées pussent continuer leurs études ou simplement suivre des cours d'enseignement général, parallèlement à l'apprentissage.

La deuxième étape a été marquée par l'organisation de l'enseignement libre moderne de langue arabe. En général, les écoles étaient mixtes et préparaient à un examen (le certificat d'études primaires des écoles libres, distinct de celui de l'enseignement public). Les enfants qui fréquentaient ces écoles appartenaient, au début, à des familles aisées, car l'enseignement était payant. Mais, petit à petit, fonctionnaires et cadres subalternes y inscrivirent leurs fillettes, et les directeurs de certains établissements admirent des enfants dont les parents ne pouvaient pas acquitter les droits de scolarité. Dans ces écoles, il n'y avait pas d'enseignement ménager; c'est dans ces établissements qu'apparurent les premières femmes institutrices, en général toutes issues de familles bourgeoises. Ces nouvelles enseignantes firent l'objet 
d'attaques et de critiques. Mais cette époque est liée à la révolution et à la lutte pour l'indépendance; c'est aussi à ce moment-là que le roi et les partis politiques prêchaient pour l'évolution de la femme et que la princesse Lalla Aïcha encourageait les femmes à prendre leurs responsabilités et à s'affranchir.

Cependant, l'enseignement d'État donné en langue française et en arabe s'organisait, de son côté ; le certificat d'études primaires était créé pour les filles ; les ateliers d'apprentissage étaient remplacés par des cours d'enseignement ménager et les fillettes accédaient au lycée, au collège, au cours complémentaire. L'enseignement technique, sanctionné d'abord par un certificat d'aptitude professionnelle, ensuite par un brevet, se substituait à l'enseignement artisanal et aux ateliers professionnels.

La troisième étape a été marquée par l'indépendance et par l'accès des femmes à la fonction publique, en nombre relativement important. Des cours de dactylographie ont été organisés en langue arabe et en langue française. Les collèges d'enseignement technique ont ouvert des cours du soir, où les femmes sont venues en assez grand nombre. C'étaient, en général, des jeunes filles qui travaillaient déjà dans un bureau et qui désiraient se perfectionner ou acquérir une qualification (sténo-dactylo).

La formation des sages-femmes et des infirmières a été lente à démarrer et à s'organiser. Cette formation a d'abord été donnée directement dans les hôpitaux, et les jeunes filles qui la recevaient n'étaient pas très bien vues; cependant, l'organisation des écoles, d'une part, les résultats obtenus par les quelques premières sages-femmes et infirmières, d'autre part, ont fait admettre ces professions. Ensuite il est devenu inconcevable qu'il n'y ait pas d'infirmières ou de sages-femmes marocaines.

L'évolution de l'enseignement et du travail féminins, ainsi que du rôle social de la femme, a eu pour conséquence de transformer la situation de celle-ci au sein de la famille. La réforme de l'enseignement et, en particulier l'organisation d'un apprentissage plus rationnel, mieux adapté aux besoins actuels, l'accès de la femme à un certain nombre de professions et sa participation à la vie de la nation ont brusquement amené les jeunes filles à disposer elles-mêmes de leur avenir. La jeune fille, comme le jeune homme d'ailleurs, prend de plus en plus d'importance dans le groupe familial et acquiert une certaine autonomie. Les parents considèrent que leur fille a plus d'expérience qu'eux-mêmes, qu'elle est davantage au courant de la vie, de l'évolution. Aussi, de plus en plus lui laisse-t-on choisir elle-même sa profession, alors que, jusqu'à ces dernières années, la décision revenait aux seuls parents. Quand elle commence à exercer sa profession, quand elle dispose d'un salaire, la jeune fille s'impose encore davantage à sa famille et acquiert au sein de celle-ci une personnalité plus marquée. 


\section{L'opinion publique devant l'accession des femmes à la vie professionnelle}

Devant cette évolution rapide du travail féminin, l'opinion publique marocaine a réagi diversement. La conception que chacun se fait du rôle de la femme se reflète dans les jugements que les différents milieux sociaux portent à l'égard de la femme qui travaille. Les professions jouissent d'un préjugé plus ou moins favorable. Enfin, indépendamment du métier exercé, la conduite et la personnalité de la femme jouent également un rôle non négligeable.

\section{a) L'opinion dans les différents milieux sociaux}

$\underline{\text { Retour à la table des matières du 2e article sur les Marocaines }}$

Dans le milieu populaire, il faut distinguer deux catégories.

Une partie de la population, assez peu nombreuse, mais d'idées très avancées, est formée d'ouvriers qualifiés, de petits employés et fonctionnaires qui prennent une part active au mouvement syndical et aux autres mouvements nationaux. L'accès de la femme au travail symbolise à leurs yeux l'évolution du pays. Ils sont favorables au travail de la femme, acceptent qu'elle accède à diverses professions ; ils en reconnaissent même l'utilité et cherchent à revaloriser les femmes qui travaillent. Mais ils exigent un comportement convenable de la part des intéressées : ils veulent qu'elles sachent se faire respecter. Leur attitude est en accord avec les opinions qu'ils professent : ils établissent des relations avec l'infirmière, l'assistante sociale ou l'institutrice qui s'occupent de leur famille et de leurs enfants. Leurs filles vont régulièrement à l'école et sont préparées à une profession : "Quand tu seras grande, disent à leur fillette les représentants de cette catégorie sociale, tu seras institutrice ou infirmière. » Quand l'intéressée atteint quinze ou seize ans, ils n'hésitent pas à consulter la directrice (ou le directeur) de l'école ou l'assistante sociale, pour demander conseil en vue de son orientation, et, si l'enfant est douée, ils sont prêts à faire des sacrifices pour lui permettre de poursuivre ses études.

Une deuxième catégorie de la classe populaire est formée des manœuvres, des petits artisans, des commerçants ambulants. Ceux-ci sont beaucoup plus réservés visà-vis de la femme qui travaille; ils émettent à son égard des critiques, qui ont pour seul fondement des motifs de ce genre : «La femme musulmane ne doit pas travailler; ce n'est pas à la femme d'entretenir la famille; les femmes qui travaillent ne sont pas sérieuses. » Les fillettes de ce milieu font leur apprentissage chez une maallema ou dans un atelier ; si elles sont scolarisées, elles suivent très vite des cours d'apprentissage artisanal. Le travail traditionnel paraît, seul, avoir de la valeur pour 
cette catégorie sociale. Il est très difficile de savoir exactement sur quoi repose l'attitude de cette importante fraction de la classe populaire, mais il est certain qu'elle est fortement influencée par le milieu traditionaliste.

Ce dernier, malgré le contrecoup des événements qui ont conduit à l'indépendance du Maroc, reste assez fort et évolue lentement. On y porte des jugements assez catégoriques contre la femme qui travaille et surtout contre celle qui travaille hors du foyer : «La femme est faite pour rester au foyer, s'occuper de son intérieur, de ses enfants. Si elle désire travailler, elle peut prendre des travaux à domicile. Le travail hors du foyer rend la femme indépendante; elle est en contact avec des hommes et devient ainsi l'égale de l'homme, alors que ce dernier doit être son supérieur. » $\mathrm{Ce}$ milieu fait souvent appel à la religion pour justifier son attitude. En réalité, il tient surtout à la tradition et ne voit dans l'accès de la femme au travail qu'un affranchissement qui risque de rompre un certain équilibre en donnant l'égalité aux deux sexes.

Le milieu formé par les cadres moyens de l'administration et par les commerçants ayant un certain niveau de vie est le plus favorable au travail de la femme. En général, c'est un milieu de jeunes où, très souvent, mari et femme travaillent. Les jeunes gens de ce milieu épousent, de préférence, une jeune fille qui travaille, ou encore, si leur fiancée est instruite en arabe ou en français, une fois mariés, ils l'encouragent à exercer une profession. Ils pensent que leur jeune épouse pourra acquérir ainsi plus d'expérience, qu'elle sera, par son travail, en contact plus étroit avec les problèmes de la vie et avec les changements de l'époque, et que l'accès des femmes au travail favorise l'évolution de la nation. Il faut aussi signaler que c'est le milieu social le plus dynamique, qui est très souvent à l'avant-garde de cette évolution.

\section{b) Prestige attribué aux différentes professions}

$\underline{\text { Retour à la table des matières du 2e article sur le Maroc }}$

Parmi les métiers exercés hors du domicile, les professions les mieux appréciées sont celles de professeur, d'institutrice, d'infirmière, de monitrice de foyer féminin. On considère que les femmes y sont à leur place et qu'elles peuvent y rendre service plus qu'un homme.

Les femmes qui travaillent dans les bureaux sont plus ou moins bien acceptées : elles sont en contact avec les hommes et cela ne plait pas. Dans certains milieux, même après avoir suivi un apprentissage de dactylographie, les jeunes filles cherchent à se diriger vers un autre métier. Les critiques les plus dures atteignent les employées des PTT. Cela est certainement dû à la promiscuité avec les hommes, qui est beaucoup plus poussée dans ce service que dans les autres bureaux, ainsi qu'aux horaires de nuit.

Les ouvrières d'usine jouissent de peu de considération, d'abord parce qu'elles font les mêmes travaux que les hommes et, en second lieu, parce qu'elles n'ont pas de qualification : «Elles sont incapables de faire autre chose », dit-on. Elles sont l'objet de critiques assez sévères, surtout si elles sont mariées. «Elles travaillent pour sortir, 
ou encore pour entretenir leur mari », disent les voisines. Dans ce cas, le couple ne jouit pas de la sympathie du quartier. Les ouvrières des usines textiles ou des fabriques de tapis sont cependant moins critiquées : d'une part, leur travail est considéré comme un véritable métier, et, d'autre part, elles sont moins appelées à travailler avec des hommes (très souvent, dans les bazars de tapis, elles ont des ateliers isolés du reste de l'entreprise).

L'opinion publique établit une hiérarchie entre les diverses professions exercées à domicile. Elle accorde la première place à celles qu'on appelle « de véritables métiers », c'est-à-dire, le plus souvent, aux métiers d'art, représentés par les brodeuses, les dentellières, les couturières, les tapissières. Mais le travail à domicile reste peu rémunéré ; aussi les couturières cherchent-elles souvent à faire autre chose, ou encore, comme à Casablanca, elles se groupent et ouvrent une boutique en ville, pour recruter une clientèle plus importante qu'à domicile. Le métier de brodeuse s'exerce de moins en moins, car le travail est long et rapporte peu. Seules les tapissières et tisseuses forment une véritable corporation; elles sont très soutenues par les inspecteurs de l'artisanat, leurs ouvrages sont soumis à l'estampillage, qui permet d'établir un classement des travaux et un ordre de prix; c'est une des professions à domicile les mieux organisées et les mieux défendues.

On apprécie beaucoup moins les métiers qui ne demandent pas une grande qualification, ceux qu'exercent les confectionneuses, les fileuses, les cardeuses de laine. Ces femmes, qui travaillent très souvent pour des commerçants ou pour des tapissières et des tisseuses, sont exposées à l'exploitation. Certaines vendent elles-mêmes leurs travaux sur les marchés. Il leur arrive fréquemment d'interrompre leur activité habituelle pour s'engager dans des travaux saisonniers, à l'usine ou à la campagne, ou pour faire des ménages.

Si les jugements portés par l'opinion publique se diversifient selon les catégories sociales et selon les professions, ils n'en tiennent pas moins compte de l'attitude personnelle de la femme au travail. Cette dernière est, de toute façon, très surveillée ; sa réputation est établie en fonction de sa conduite, de ses relations, de ses réactions dans son travail et hors de son travail. La façon dont elle s'impose à son entourage, à ses collègues, au public, à sa clientèle compte beaucoup. Les femmes qui travaillent hors de leur domicile sont les plus exposées à la surveillance, à la critique et, très souvent, des jugements hâtifs sont portés sur elles, pour un simple geste, pour un simple mot. Le milieu familial a beaucoup d'importance; si sa famille jouit d'une bonne réputation, la jeune femme ou la jeune fille est à l'abri de certaines critiques et bénéficie d'une certaine confiance de la part des gens.

En conclusion, si le travail de la femme - et surtout le travail hors du domicile commence à être admis, on doit reconnaître qu'il ne l'est qu'avec beaucoup de réserves. On s'incline, parce que l'émancipation de la femme est devenue un symbole de l'évolution générale du pays, et on en proclame la nécessité. Mais il reste encore bien des obstacles à franchir - aussi bien ceux que dressent les anciens, encore hostiles à l'émancipation des femmes, que ceux qui viennent des réserves des jeunes : théoriquement, ceux-ci désirent cette émancipation, mais, pratiquement, ils la craignent. 
Images de la femme dans la société

\section{IV \\ Trois études préliminaires : Canada, Côte d'Ivoire, Togo}

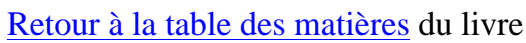

À la suite des premières enquêtes qui viennent d'être présentées, une nouvelle phase de recherche s'est ouverte devant nous. Les comparaisons Est-Ouest et les comparaisons Europe-Afrique sont incomplètes. Comment vont-elles se préciser, sur quels terrains, en fonction de quel plan?

Plusieurs réponses sont données ici, pour le Canada, la Côte-d'Ivoire, le Togo, l'Autriche, la Yougoslavie. Pour chaque pays, de nouveaux problèmes d'orientation et de méthode se posent, bien que demeure inchangé l'axe de recherche choisi au départ. En lisant ces essais, le lecteur pourra suivre le dialogue qui s'est établi entre les chercheurs appartenant à des cultures différentes.

Comme nous l'avons signalé dans l'introduction, les textes de Mme Burié, de L. Rosenmayr et de R. Clignet utilisent les données d'enquêtes particulières, entreprises parallèlement à l'enquête principale. En fonction des questions posées, ces auteurs ont introduit un aspect nouveau dans le développement des recherches, en exprimant leur point de vue sur l'étude des images et de la perception de l'évolution. G. Rocher, qui a participé à la préparation de l'enquête internationale dès l'origine, donne les grandes lignes d'un programme canadien et précise, lui aussi, ses positions sur le problème de l'étude du statut de la femme.

Les enquêtes portant sur l'Afrique noire, telles qu'elles sont proposées par $\mathrm{N}$. Agblemagnon, ou déjà en partie réalisées par R. Clignet et l'équipe de Côte-d'Ivoire, devront être rattachées à l'ensemble des travaux poursuivis depuis longtemps par d'autres chercheurs sur l'évolution de la femme dans la vie rurale et la vie urbaine par exemple, aux travaux des congrès féminins qui se sont tenus en Afrique, aux études parues récemment sous la direction de D. Paulme, aux remarques contenues dans l'ouvrage publié sous la direction de D. Forde, etc. Comme pour le Maroc, nous 
verrons rapidement de jeunes sociologues africains prendre en charge des recherches portant sur leur propre pays.

I. Guy Rocher, "Les modèles et le statut de la femme canadienne-française"

II. R. Clignet, "Les attitudes de la société à l'égard des femmes en Côted'Ivoire"

III. F. N'Sougan Agblemagnon, "Recherche sur les attitudes vis-à-vis de la femme togolaise" 
IV

Trois études préliminaires: Canada, Côte d'Ivoire, Togo

\section{I. \\ "Les modèles et le statut de la femme canadienne-française" par Guy Rocher [sociologue, Université de Montréal]}

[texte reproduit avec l'autorisation de l'auteur]

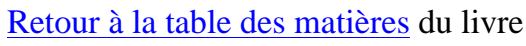

Table des matières de la $1^{\text {re }}$ étude préliminaire: les femmes canadiennes

1. Le travail professionnel et la femme canadienne-française

2. La femme canadienne-française et la vie politique

3. Le statut de la mère canadienne-française

Pour qui vit de l'intérieur les transformations sociales que connaît le Canada français, il est évident qu'il s'y produit de profondes modifications en ce qui concerne le statut de la femme. Les modèles de la femme canadienne-française avaient été, auparavant, la mère, la religieuse et l'institutrice. C'était donc dans la famille, l'éducation de la jeunesse et les oeuvres de bienfaisance qu'elle trouvait ses champs d'activité exclusifs et que se définissaient ses rôles. Elle n'avait part ni à la vie économique, ni à l'action politique du pays. On disait couramment que «la politique était une histoire d'hommes » et que les femmes « ne comprenaient rien aux affaires »; ce sont là des expressions qui vivent encore dans notre folklore.

Depuis le début du siècle, cependant, une profonde évolution s'est manifestée dans le statut de la situation de la femme canadienne-française. Mais aucune étude n'a encore cherché à donner une vision de ce processus en quelque sorte à travers les yeux de ceux qui le vivent, c'est-à-dire en analysant si et comment les changements sont perçus par les femmes elles-mêmes, quelles réactions ils provoquent chez les 
hommes, quelles nouvelles attitudes communes ou différentes s'élaborent. S'il est vrai que la femme participe davantage à l'activité économique ou politique du pays, peuton dire que l'ensemble de la société canadienne-française en a pris conscience ? Cette conscience serait-elle plus vive dans certaines couches horizontales ou certaines sections verticales de la population ? Quelle signification attribue-t-on à ce changement et comment le juge-t-on?

Il est très probable qu'on n'observerait ni le même état de conscience, ni les mêmes attitudes dans les différents groupes et les différentes catégories de la population. Tout d'abord, les changements n'ont touché que certains secteurs de la société, par exemple le secteur urbain plus que le secteur rural, certaines régions plus que d'autres. De plus, même dans une ville comme Montréal, toutes les couches de la société ne sont pas prêtes à réagir de la même façon à un changement de cette nature ; chacune l'assimile en fonction d'un contexte socio-culturel qui lui est propre. Le statut et les rôles de la femme ayant été définis avec des nuances différentes chez les ouvriers et les membres des professions libérales, les changements ne seront pas interprétés de la même façon.

Ce sont là autant de questions auxquelles nous n'aurons pas de réponse à donner, aussi longtemps qu'une étude en profondeur, du genre de celle qui a été entreprise, par exemple, en France par le Groupe d'ethnologie sociale, n'aura pas été faite chez nous. Seule une telle étude permettrait de comprendre, par l'intérieur, les changements en cours, d'en saisir la signification et d'en prévoir peut-être les orientations futures.

Dans l'attente de cette recherche, nous ne pouvons, pour le moment, que décrire le contexte dans lequel elle se déroulerait, c'est-à-dire formuler certaines hypothèses sur la base des renseignements objectifs que nous possédons déjà ou des simples impressions qui tiennent encore lieu de conclusions. Nous présenterons donc quelques brèves observations, qui permettront de saisir certains traits de l'évolution actuelle, en particulier dans trois secteurs : le travail professionnel de la femme canadienne-française, sa participation à la vie politique et le statut de la mère dans la famille moderne.

\section{Le travail professionnel et la femme canadienne-française}

$\underline{\text { Retour à la table des matières de l'article sur les femmes canadiennes }}$

L'aspect sous lequel le changement est le plus frappant, du moins à première vue, est la part grandissante de la femme dans la population active. Quelques chiffres aideront à préciser ce phénomène. Au recensement de 1901, le pourcentage de la population féminine de dix ans et plus qui exerçait une activité dans la province de Québec (province française du Canada) s'élevait à 15\% - ce qui représentait un peu plus du dixième de l'ensemble de la main-d'œuvre de la province. Cette proportion était à peu près la même pour l'ensemble du Canada, Depuis ce temps, le pourcentage de la population féminine de quatorze ans et plus qui exerce une activité n'a cessé de 
s'accroître de façon constante, pour s'élever à 28\% à la fin de l'année 1960 - soit plus du quart de l'ensemble de la main-d'œuvre du Québec ${ }^{1}$, tandis que le nombre de femmes mariées employées dans les industries non agricoles du Canada a presque triplé de 1946 à $1959^{2}$.

C'est dire qu'au début du siècle les femmes ne travaillaient que dans les cas de nécessité (veuvage, séparation, maladie du chef de famille, célibat). De façon assez générale, quoique peut-être plus encore dans les familles «bourgeoises » en particulier, les normes sociales dominantes ne permettaient pas qu'une jeune fille travaillât « en attendant de se marier » ou qu'une mère de famille songeât à s'embaucher sans raisons financières impérieuses. On doit constater que la mentalité a très fortement changé sur cette question. L'évolution a été si marquée, particulièrement en ce qui concerne la jeune fille, que, dans toutes les couches de la société canadienne-française, on considère comme normal qu'elle travaille, au moins jusqu'à son mariage. La jeune fille ne confectionne plus de ses mains son trousseau de mariage elle le gagne par son travail.

L'habitude se répand aussi, pour la jeune femme, de continuer à travailler après son mariage, jusqu'à ce qu'elle ait un ou deux enfants. Cette habitude s'observe surtout aux Etats-Unis d'Amérique et chez les Canadiennes anglaises. Elle se répand cependant beaucoup plus lentement chez les femmes canadiennes-françaises. Un fait est très frappant à cet égard. Au recensement de 1951, la province de Québec venait en huitième position parmi les dix provinces canadiennes pour la proportion de femmes mariées occupant un emploi rémunéré, alors qu'elle était en deuxième position pour la proportion des femmes dans l'ensemble de la main-d'œuvre. C'est dire que, dans la province de Québec, le nombre de femmes qui travaillent est élevé par rapport au reste du Canada, mais qu'il s'agit, pour la très grande majorité, de jeunes filles. Ce qui amenait les auteurs d'une étude sur le travail de la femme canadienne, publiée par le Ministère du travail du Canada, à conclure en ces termes : «Il est à remarquer que, dans les provinces où une proportion élevée de l'ensemble des femmes travaillent, la proportion des femmes mariées en emploi est également susceptible d'être élevée. Il n'y a pas de doute que la disponibilité d'emploi joue ici un rôle important. Le Québec est l'exception frappante à cette règle générale. C'est une province hautement industrialisée, et une proportion de femmes plus élevée que pour l'ensemble du Canada y travaillent ; cependant, la proportion des femmes mariées en emploi hors du foyer est bien au-dessous de la moyenne nationale. Ce fait résulte des traditions sociales et culturelles de la province $»^{3}$.

Ce sont précisément ces «traditions sociales et culturelles » qu'il faudrait pouvoir expliciter, puisqu'on leur attribue une telle importance dans l'explication du comportement «exceptionnel » des femmes québécoises. Que recouvre exactement cette expression ? Seule une étude détaillée des attitudes du milieu canadien-français à l'endroit du travail féminin permettrait d'apporter plus de contenu à cette explication trop vague.

Nous pouvons cependant ajouter que certains points de repère nous sont déjà fournis. Une étude faite récemment par le Ministère canadien du travail portant sur un échantillon de femmes mariées qui occupaient un emploi rémunéré, dans huit villes

La main-d'œuvre, Bureau fédéral de la statistique, décembre 1960, J. 4.

Ibid., septembre 1960, J. 2.

Ministère du travail du Canada, La femme canadienne au travail, 1958, pp. 19-21. 
du Canada, a révélé que la femme québécoise qui continuait de travailler après son mariage tendait à se retirer de la population active lorsqu'elle avait des enfants, à moins d'être le seul ou le principal gagne-pain de la famille. Une plus forte proportion de femmes mariées canadiennes-anglaises gardent leur emploi lorsqu'elles ont de jeunes enfants ou reviennent travailler lorsque leurs enfants ont atteint l'âge scolaire ${ }^{1}$. D'autre part, on a constaté, dans la même étude, que le motif le plus fréquemment invoqué par les femmes pour expliquer leur travail était le besoin économique. Cependant, les auteurs observent : "Le motif de beaucoup le plus courant pour expliquer le travail des femmes hors du foyer - surtout des femmes ayant des enfants consiste dans le désir de hausser le niveau de vie familial. Dans quelques cas, la contribution de la femme change sensiblement les choses, doublant presque le revenu familial ; mais, dans le cas d'un grand nombre de familles, les gains de la femme permettent de se procurer du « superflu », dont autrement on se serait passé ${ }^{2}$ » Les auteurs considèrent comme assez caractéristique cette réponse de l'une des femmes interviewées : "L'argent supplémentaire constitue toute la différence entre l'obligation de ménager et la liberté de s'accorder quelque superflu ${ }^{3} \gg$.

Nous touchons ici un intéressant problème de valeur. Il est certain que les familles canadiennes-françaises ne jouissent pas d'un revenu supérieur à celui des foyers canadiens-anglais. L'option se ferait donc entre le confort supplémentaire qu'apporte le travail de la femme et le rôle traditionnel de la femme dans la famille, et c'est cette option qui tendrait à être différente dans les deux milieux. Les Canadiens-Français accorderaient à la place de la femme au foyer et à son rôle auprès des enfants une valeur qui contre-balancerait les aspirations à un niveau de vie plus élevé. Telle est, du moins, l'hypothèse que permettent de formuler les données que nous possédons actuellement. Seule une étude plus qualitative et détaillée permettrait d'éclairer cette hypothèse et de lui donner un contenu.

\section{La femme canadienne-française et la vie politique}

$\underline{\text { Retour à la table des matières de l'article sur les femmes canadiennes }}$

La femme canadienne-française jouit, depuis 1940, de droits politiques égaux à ceux de l'homme. Elle a le droit de vote pour l'élection du gouvernement provincial et est éligible à toutes les fonctions gouvernementales. L'histoire de l'obtention de ces droits méritera cependant d'être écrite ; elle constitue un exemple typique des résistances d'une société traditionaliste à une innovation dangereuse. On peut dire, en effet, que ces droits furent finalement acquis après trente années d'une lutte soutenue et tenace.

Ministère du travail du Canada, Les femmes mariées en emploi rémunéré, 1958, p. 63.

Ibid., p. 54

Ibid., p. 56. 
Dès 1918 , le gouvernement canadien avait octroyé le droit de vote aux femmes pour les élections fédérales et leur avait permis l'accès aux fonctions gouvernementales. Mais le gouvernement de Québec demeura opposé à un projet semblable au niveau provincial durant vingt-deux ans - et cela, en dépit d'une intense activité de la part de diverses associations féminines. Le Comité provincial du suffrage, la Ligue pour les droits de la femme, l'Alliance canadienne pour le rôle des femmes de Québec et d'autres groupements réussirent à faire présenter, à chaque session parlementaire, un projet de loi, qui aboutit chaque fois au même échec. Une génération de femmes actives et intelligentes s'est usé les dents à cette lutte désespérante contre le préjugé et la passivité ${ }^{1}$.

On peut d'ailleurs dire que le droit de suffrage fut finalement accordé aux femmes de Québec grâce, surtout, à une conjoncture politique favorable (la victoire du parti libéral de M. Godbout, en 1939), bien plus que par suite d'un vaste mouvement populaire. Il est, en effet, remarquable que la province de Québec n'ait jamais élu une seule femme ni au gouvernement provincial, ni au gouvernement fédéral, alors que la chose s'est produite dans presque toutes les autres provinces canadiennes. L'actuel gouvernement fédéral compte même une Canadienne-Anglaise parmi les ministres du cabinet. Sans doute les femmes ont-elles assez activement participé à la vie politique et aux élections de ces dernières années. Ainsi, le chef provincial du parti CCF (Parti socialiste) a été, durant plusieurs années, une femme, Mme Pierre Casgrain, qui avait auparavant pris une part très active aux luttes pour l'obtention du droit de vote. De même, le parti libéral a créé, en 1950, une Fédération des femmes libérales du Québec, qui déploie une activité grandissante. Mais, avec l'obtention du droit de vote, la lutte contre le préjugé et la passivité n'a pas été terminée - c'est peut-être chez les femmes elles-mêmes qu'elle doit maintenant se poursuivre, pour développer chez elles une conscience politique plus éclairée et plus aiguë. Les intéressées n'ont pas encore assimilé le statut égalitaire qui leur a été octroyé et n'ont pas su lui donner sa pleine réalité.

Il faudrait ajouter ici que, si la femme canadienne-française a acquis l'égalité des droits politiques, elle est encore considérée comme une mineure pour ce qui concerne les droits juridiques. Le code civil de la province de Québec, qui est calqué sur le code Napoléon et n'a pratiquement pas connu d'amendement depuis 1866, accorde au mari tous les droits juridiques. Sans l'autorisation du mari, la femme ne peut ni acheter, ni faire ou accepter une donation, ni commercer, ni intenter d'action, ni contracter, ni administrer ses biens meubles et immeubles. Nous sommes donc dans cette situation paradoxale : la femme canadienne-française peut être élue pour administrer les biens de la nation, mais elle ne peut gérer les affaires de son ménage.

Ce paradoxe est bien l'indice d'une situation en pleine évolution.

Mme Florence F. Martel, qui participa activement à ces luttes, en donne un bref historique dans un article intitulé : «Il y a vingt ans, les libéraux accordaient le droit de vote à la femme du Québec », La réforme, 23 avril 1960. 


\section{Le statut de la mère canadienne-française}

$\underline{\text { Retour à la table des matières de l'article sur les femmes canadiennes }}$

Cette évolution n'affecte pas seulement la femme dans ses rôles à l'extérieur du foyer; elle touche aussi son statut dans la dynamique même des relations familiales. L'analyse doit ici se faire plus subtile et nuancée, car nous ne sommes plus en présence d'un phénomène aussi aisément observable que les précédents. Ce n'est plus à l'échelle de l'ensemble de la société, mais à celle de la vie familiale quotidienne que les observations doivent être recueillies. De plus, l'étude diachronique est beaucoup plus difficile, parce que les documents nous manquent pour nous renseigner sur le statut antérieur de la mère canadienne-française. Il faut se fier trop souvent au témoignage déformant de la mémoire.

On peut cependant facilement observer une certaine ambiguïté de l'univers culturel canadien-français en ce qui concerne la mère. D'une part, notre notion traditionnelle de la famille a toujours mis l'accent sur l'autorité paternelle. Le père est décrit comme le chef de la communauté familiale, la figure dominante, entourée de respect et de soumission. La mère, tout autant que les enfants, est subordonnée à cette autorité. Dans la division des tâches, le père est exempté des travaux domestiques ; la femme sert son mari à table et dans la maison, où l'homme préside en grand seigneur. C'est à l'extérieur du foyer que celui-ci déploie son activité ; la maison est le lieu de son repos et le royaume sur lequel il règne ${ }^{1}$.

Mais, en même temps, la figure de la mère est hautement valorisée comme étant l'âme du foyer, sur qui reposent l'unité et la solidité de la famille. C'est elle qui assure l' « esprit de famille », auquel on attache une très grande importance. Elle est considérée comme le lien qui unit entre eux, grâce a elle, les membres de la communauté. C'est sur le plan des relations affectives que la mère joue un rôle dominant; elle resserre les liens familiaux par les rapports d'amour et d'affection qu'elle maintient et catalyse.

Ainsi, sans reconnaître à la mère l'autorité formelle qui est dévolue au père, on lui en attribue tous les pouvoirs. Soumise au mari en apparence, elle tient en main les fils essentiels de l'autorité parentale. C'est par la puissance des liens affectifs qu'elle régit la communauté familiale, sous l'autorité officielle du père. C'était d'ailleurs réduire celui-ci à n'avoir aucun pouvoir réel dans la maison que de le servir. Notre folklore familial connaît bien cette sorte de complicité des femmes et des enfants, pour contourner ou diminuer l'autorité paternelle.

C'est dans le contexte de cette image de notre famille traditionnelle que se produisent les changements qui affectent le statut de la mère. La famille moderne se veut plus «égalitaire », c'est-à-dire qu'elle veut accorder à la femme un statut qui ne soit plus fait de soumission et de subordination. Dans la jeune famille canadienne-

1 Guy Rocher, « Le père », Food for thought, vol. 14, no 6, mars 1954. 
française, le mari n'apparaît plus comme le chef que l'on sert. Il doit prendre une part beaucoup plus active aux travaux domestiques et à l'éducation des enfants. Le mari partage aujourd'hui beaucoup plus avec sa femme les préoccupations, les fatigues et les joies de la vie domestique et de l'éducation; lui-même se veut présent dans l'univers de ses enfants, non seulement comme y représentant l'autorité, mais aussi par les liens affectifs établis avec eux.

Une des conséquences de ces transformations, c'est de rompre le très subtil équilibre des pouvoirs au sein de notre famille traditionnelle. Ce qu'a perdu le père en autorité officielle, il semble le retrouver en pouvoir réel, de sorte que l'égalitarisme de notre famille moderne entraîne peut-être finalement un affaiblissement de l'emprise que la mère exerçait auparavant : elle ne domine plus la dynamique complexe des relations entre les membres de la famille.

L'évolution de notre famille moderne impose ainsi à la mère non seulement de nouvelles conditions de travail (logement, appareils ménagers, horaire, etc.) et de nouvelles responsabilités (civiques, politiques, professionnelles), mais aussi un statut bien différent de celui qu'elle a connu dans le passé. Vu sous son aspect négatif, ce nouveau statut se traduit peut-être surtout par le fait que la mère n'est plus la figure dominante de la vie familiale quotidienne.

L'analyse qui précède peut étonner. Nous croyons, cependant, qu'elle devrait constituer l'une des hypothèses directrices pour l'étude du statut de la femme. La vie familiale a connu, au Canada français, des transformations si profondes que le statut de la mère doit être radicalement mis en question, au moins dans l'élaboration des hypothèses de travail, si l'on veut mesurer l'évolution et suivre le processus de changement.

C'est l'état présent de cette évolution que permettrait de saisir une analyse en profondeur de la perception du statut de la femme. Nous croyons qu'une telle étude ferait apparaître les contradictions entre la conception traditionnelle de la femme, avec son imagerie et son folklore, et la nouvelle figure de la femme canadiennefrançaise, dans le contexte des structures sociales en cours de transformation. Par cette étude de perception, nous rejoignons ici, comme à travers l'idéologie de Mannheim *, les points de rupture dans une organisation sociale en voie d'évolution rapide. C'est cette organisation qui se reflète, en même temps qu'elle se construit, dans et par les attitudes et les perceptions. Le statut de la femme constitue l'un des points névralgiques dans la transition d'une société traditionnelle à une société industrielle moderne. Ce passage suppose une profonde modification à la fois des catégories mentales à travers lesquelles est perçue la femme et des normes ethniques selon lesquelles elle est jugée. L'étude de la difficile révision de ces catégories et de ces normes imposée à la conscience d'une société permet de saisir, en quelque sorte par l'intérieur, de «comprendre »- au sens allemand de verstehen - les changements de structure et les brisures qu'ils entraînent dans un milieu en rapide évolution.

Voir Idéologie et utopie. Une introduction à la sociologie de la connaissance (1929), de Karl Mannheim, disponible en version intégrale dans Les Classiques des sciences sociales [Note : JMT] 
IV

Trois études préliminaires: Canada, Côte d'Ivoire, Togo

\section{II \\ "Les attitudes de la société à l'égard des femmes en Côte-d'Ivoire"}

par R. Clignet

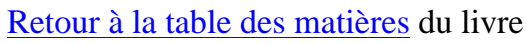

La promotion de la femme africaine est devenue un leitmotiv des discours politiques, des sermons religieux et des déclarations de chefs d'entreprise. Une telle insistance reflète l'image de l'électrice, de la mère, de la salariée. Si les pouvoirs publics, occasionnellement aidés par des initiatives privées, ont ouvert de nombreuses écoles en vue de faire des femmes des maîtresses de maison et des ménagères accomplies, des cadres sociaux destinés à l'enseignement, aux hôpitaux et à des services tels que les affaires sociales, le secteur privé n'accepte que très partiellement d'utiliser de la main-d'œuvre féminine : si la «femme » est utilisée comme domestique au Gabon, si elle est utilisée comme hôtesse de l'air dans la même région, si elle est employée comme ouvrière dans une usine d'allumettes de Dakar, elle doit se contenter, pour le moment, de faire une entrée encore discrète et timide dans certains emplois de bureau du secteur privé de la Côte-d'Ivoire. Certes, l'administration utilise ses compétences dans l'enseignement, les services sociaux et hospitaliers. Mais les restrictions dont on fait preuve, en ce qui concerne son emploi, semblent indiquer qu'à des résistances propres à la société ivoirienne viennent s'ajouter des stéréotypes d'importation européenne.

Ces stéréotypes se retrouvent d'ailleurs au niveau des modèles retenus pour l'établissement des écoles de formation ménagère ou sociale. Ces modèles sont ambigus. Ils trahissent, la plupart du temps, la persistance d'un mythe assimilationniste. Dans le souci de donner aux femmes africaines des droits, une culture, une éducation équivalents à ceux de la femme métropolitaine, on oublie que cette démarche représente un mouvement d'adaptation qualitativement et quantitativement 
beaucoup plus important pour les intéressées que pour leurs homologues métropolitaines. Le terme d'assimilation - intégration de la réalité à un schéma préétabli - est sollicité par une confusion sociocentrique, puisque son contenu évoque beaucoup plus l'idée d'accommodation, c'est-à-dire d'une modification des schémas de la pensée traditionnelle aux prises avec les valeurs et la situation européennes ${ }^{1}$.

Cette confusion résulte d'ailleurs partiellement de la rotation trop fréquente du personnel chargé de la formation des élites locales ; cette rotation entraîne, en effet, une ignorance des valeurs, des modèles et des stéréotypes des sociétés constitutives de la nation ivoirienne. Cette ignorance ne peut être compensée que par la négation de la spécificité ivoirienne. Il n'est, en fait, pas dit que les mesures assimilationnistes soient souhaitées par les intéressées.

Toute réponse rationnelle au problème de la promotion de la femme suppose un inventaire préalable des attitudes adoptées à l'égard de celle-ci. De là l'intérêt pratique de l'enquête proposée par le professeur Chombart de Lauwe. De plus, cette enquête menée longitudinalement (dans divers pays) et transversalement (en fonction des variables stratégiques constitutives de l'acculturation) permet éventuellement d'esquisser une analyse théorique des relations entre les structures sociales et cette catégorie d'attitudes.

L'approche d'un tel problème suppose donc une description préalable des phénomènes relevant de cette sphère dans la société traditionnelle, pour les confronter ensuite aux principaux facteurs qui ont le plus affecté la société ivoirienne.

La plupart des anthropologues ont insisté sur la primauté de la famille nucléaire dans toutes les sociétés humaines ${ }^{2}$.

Les caractéristiques de ce groupe sont définies comme étant la communauté de résidence, la coopération économique de ses membres et la reproduction. Murdock distingue, au sein d'un tel groupe, huit types de relations interpersonnelles ${ }^{3}$, parmi lesquelles on peut accorder une place privilégiée aux relations unissant le mari et la femme.

Ces relations comprennent l'ensemble des mécanismes de contrôle et de régulation des satisfactions sexuelles. Mais elles constituent surtout un aménagement dans le système de répartition de la nourriture au sein du groupe dans lequel s'insère la famille nucléaire ${ }^{4}$. De ce fait, le mariage apparaît moins comme un accord entre un homme et une femme que comme une relation globale d'échanges entre deux groupes d'hommes, la femme n'étant qu'un des objets de ces échanges généralisés. Le poids relatif de la sexualité et de la coopération économique dans les relations inter-époux dépendrait ainsi de l'importance des déséquilibres alimentaires dans la société globale.

Ces termes, issus de l'œuvre de Piaget, sont définis par Lagache dans Bull. de Psych. vol. 9, p. 531.

2 Löwie, Sociologie primitive, p. 76 ; Murdock, Social structure, p. 1.

3 Ibid., p. 3.

4 Lévi-Strauss, Structures élémentaires de la parenté, p. 48. 
Ainsi apparaissent deux dimensions importantes du rôle attribué à la femme ivoirienne : la nature des tâches qui lui sont demandées et la reproduction, destinée à assurer une main-d'œuvre plus abondante, nécessaire à une agriculture extensive.

À l'origine, surtout dans l'ouest du pays, les activités de cueillette, de chasse et de pêche étaient le fait des hommes, tandis que les femmes devaient assurer l'ensemble des activités domestiques (cuisine et activités annexes, soins donnés aux enfants) et des tâches agricoles - celles-ci ne concernant que les cultures vivrières. Situant ce phénomène dans un contexte plus large, Murdock ${ }^{1}$ l'interprète en soulignant que la première catégorie de travaux suppose une connaissance approfondie des terrains appartenant au groupe et que cette connaissance est un apanage masculin.

Dans l'est du pays, l'homme s'est trouvé associé plus tôt à des activités agricoles telles que la culture du palmier. Dans le cadre de cette culture ${ }^{2}$, la femme n'en reste pas moins attachée à des tâches qui ne l'éloignent pas du village : cultures vivrières, traitement des oléagineux.

De la même manière (tant à travers les institutions qu'à travers les formes de sociabilité spontanées), la fécondité apparaît être une valeur essentielle de la femme. La richesse du droit en la matière, la complexité des rites et des cures pour éviter la stérilités ${ }^{3}$ en témoignent. Interviewées par un groupe d'élèves de l'École normale ménagère, la grande majorité d'un échantillon expérimental de 213 femmes de la Basse-Côte voient, dans le fait d'avoir des enfants, un avantage essentiel du mariage.

Si la femme est perçue comme source de travail et comme procréatrice d'enfants donc comme source de richesse - elle est aussi, en tant qu' « objet de communication », signe de richesse. Ainsi apparaissent deux facteurs constitutifs de la polygynie.

Cette forme de famille, cependant, est liée à une conception particulières des structures politiques et économiques ${ }^{4}$ de la société globale. Murdock insiste ainsi sur l'importance de la corrélation entre cette institution et la concentration des pouvoirs politiques et financiers entre les mains des hommes.

Le même auteur constate également que la règle patrilocale de résidence 5 augmente les probabilités de la polygynie; ainsi s'expliquerait le fait que, quel que soit le système de transmission des biens en vigueur dans les groupes constitutifs de la société ivoirienne (matrilignage ou patrilignage), le taux de polygynie soit partout le même. Tous les groupes, en effet, suivent cette règle de résidence.

Dans une telle perspective, la polygynie semble donc liée à une relative réussite matérielle que, dans la majorité des cas, l'âge seul permet d'atteindre ${ }^{6}$. De là vient la différence d'âge sensible entre mari et femme, le nombre de femmes disponibles dans la classe d'âge du mari et ce qu'elles représentent ne pouvant permettre la satisfaction des besoins de prestige nourris par l'intéressé.

Social structure, p. 213; et Löwie, dans : Oceanic monographs, no I, p.. 31.

Boutiller et Dupire, L'homme adioukrou et sa palmeraie.

Kerharo, Sorciers et féticheurs de la Côte-d'Ivoire.

Löwie, op. cit., p. 130 et suiv.

Murdock, Op. cit., p. 207.

Mercier, dans : Cahiers d'études africaines, no 1, p. 39. 
Cette importante différence d'âge infléchit le type des relations interpersonnelles entre mari et femme : le mariage doit apparaître beaucoup plus comme un mariage institutionnel que comme un mariage-compagnonnage ${ }^{1}$. Au sein du même échantillon expérimental étudié en Côte d'Ivoire, il semble que l'autoritarisme résultant de cette situation soit accepté par les femmes. Le degré de concurrence entre les diverses générations d'hommes pour l'acquisition des femmes détermine, cependant, le niveau d'acceptation de cet autoritarisme par les femmes.

Ainsi apparaissent des différences entre les sociétés patrilinéaires de l'ouest du pays (Bétés) et les sociétés matrilinéaires (Agnis-Baoulés, Abourés, etc.) : au sein des premiers groupes, la concurrence acharnée à laquelle se livrent les hommes pour acquérir une femme - concurrence exprimée par le montant élevé de la contreprestation matrimoniale -entraîne une tendance accentuée des femmes à rechercher et à obtenir une indépendance de fait. Il n'en va pas de même dans le deuxième groupe, où la femme semble plus disposée à accepter la tutelle à laquelle elle se trouve soumise. La signification de ces différences, mesurées sous l'angle de l'âge réel du mari, du montant de la contre-prestation et des attitudes de la femme vis-à-vis de ces deux variables, fait actuellement l'objet d'un test portant sur un échantillon de 1100 femmes bété et 800 femmes abouré.

La situation inférieure de la femme apparaît encore à travers le système de transmission des biens. Quel que soit le système choisi, matrilignage ou patrilignage, la femme demeure « objet de communication et d'échanges ». L'illustration la plus frappante de ce phénomène est l'institution du lévirat (obligation pour l'héritier d'épouser la veuve du de cujus), que l'on rencontre dans les deux types de société en Côte-d'Ivoire. Cette institution semble cependant plus répandue dans le groupe bété (patrilinéaire), que dans le groupe abouré (matrilinéaire), où la femme, à la mort de son époux, semble être souvent revendiquée par son groupe d'origine.

C'est que les échanges ont un caractère précaire ; chacun des époux reste attaché à son groupe d'origine - ce qui entraîne sans cesse des conflits de loyauté ${ }^{2}$. De nombreux auteurs - Radcliffe Brown, notamment - insistent sur le fait que, dans les sociétés matrilinéaires et, en particulier, chez les Ashantis, les confidences sont beaucoup plus importantes entre frères et sœurs qu'entre époux ${ }^{3}$. La forme la plus courante de communication entre époux est l'indifférence. De la même manière, la femme bété doit toujours prendre le parti de son lignage contre son mari ${ }^{4}$. Les décisions les plus importantes de la vie familiale sont, en tout état de cause, prises par le chef de lignage ou le mari.

Ainsi, les principes de division du travail, la fertilité, les règles de mariage, de résidence et d'héritage constituent les principales variables de la société traditionnelle, significatives de l'infériorité du statut accordé à la femme. Si les valeurs sous-jacentes à certaines de ces variables apparaissent acceptées par les femmes (fécondité, différence d'âge), il n'en va pas de même pour toutes. En face des groupes d'hommes, les femmes ont leur dynamisme et leurs formes de groupement propres. Imposé par

Burgess, Recherches sur la famille, de J. C. B. Mohr, p. 69 et suiv.

Denise Paulme, dans -. Cahiers d'études africaines, no 1, p. 26 et suiv.

Systèmes matrimoniaux en Afrique noire, p. 360.

Kobben, Le planteur noir, études éburnéennes, p. 102. 
leurs partenaires ${ }^{1}$ le contexte dans lequel se situent les attitudes à l'égard du statut de la femme est donc originellement différent du contexte européen.

L'acculturation a eu pour effet de modifier nombre de ces variables. Il semble donc indispensable d'en cerner avec précision les contours essentiels, en fonction du problème posé.

En Côte-d'Ivoire, l'introduction des cultures industrielles a bouleversé l'équilibre et les structures des divers groupes sociaux. Modifiant les régimes de propriété dans le sens d'une individualisation progressive, elle a également modifié les règles de division du travail ${ }^{2}$. De l'activité de cueillette ou de chasse, l'homme est passé à la culture vivrière, très rapidement, pour aboutir à la plantation du café ou du cacao.

Le grand principe de division du travail en fonction de la connaissance du terrain est demeuré : les hommes débroussent et défrichent le terrain de la plantation et se chargent de la plantation proprement dite. Les femmes s'occupent des cultures vivrières, à proximité du campement ou du village : elles interviennent sur les plantations seulement pour le désherbage et la cueillette. Leur rôle, par contre, est fondamental en ce qui concerne le séchage, le décortiquage et le triage des deux genres de produits. Il en résulte une possibilité de tension entre les époux. En effet, si la règle traditionnelle de division du travail ne posait aucun problème dans le cadre d'une économie de subsistance, il n'en va plus de même dans le cadre d'une économie de profit. Boutiller ${ }^{3}$ et Dupire ont parfaitement analysé les tensions créées par l'introduction d'une huilerie dans la région de Dabou, au sein du groupe adioukrou. Les revenus de la femme étaient, jusqu'à ces dernières années, assurés par sa participation aux cultures industrielles (elle était payée comme salariée, par son mari, sur la base de 2 pagnes par an); par la vente, au marché, de produits des cultures vivrières; et surtout par la fabrication et la vente, dans les mêmes conditions, d'huile tirée des palmiers.

Traditionnellement, donc, si la femme assurait le transport et la transformation des régimes, l'homme soignait la plantation et assumait la majorité des tâches liées à la récolte. La mise en place de l'huilerie a abouti à l'enlèvement direct des régimes, sur les plantations, par des véhicules appartenant à l'entreprise. Dans la mesure où l'extraction de l'huile était assurée, dans la proportion de $80 \%$ par les femmes, il en résulte un déséquilibre considérable dans la distribution des revenus entre les deux sexes. Dans le domaine du palmier, la seule rémunération dont la femme peut disposer présentement résulte d'activités de transport, et cette rémunération n'offre aucune garantie; il est donc explicable que soient remis en cause les principes traditionnels de division du travail.

Pour des raisons analogues de profit, Binet ${ }^{4}$ constate qu'au Cameroun certaines femmes revendiquent des cacaoyers.

L'économie de profit a également modifié la structure des groupes qui ont introduit ces cultures industrielles. Mais une telle modification ne joue pas partout dans le même sens. Kobben, dans Le planteur noir, montre ainsi que la société agni, dans l'ouest du pays, a rapidement adopté les valeurs et les techniques de la libre entre-

Binet, « La femme et l'évolution de l'Afrique », Revue de l'action populaire, juin 1960, p. 747.

Kobben, op. cit.

Op. cit., dernier chapitre.

Op. cit., 13, 752. 
prise. Le bénéfice tiré de l'exploitation de la terre est réinvesti dans l'achat de terrains, dans la location de main-d'œuvre étrangère et dans l'amélioration des conditions d'habitat ${ }^{1}$. De ce fait, les signes traditionnels de richesse et de prestige commencent à perdre leur signification.

Le même bénéfice, dans la société bété, sert à satisfaire le «complexe de femmes ». L'introduction des produits manufacturés a entraîné une augmentation considérable des contre-prestations matrimoniales. L'accroissement de la circulation monétaire coïncidant avec des conflits assez violents entre les diverses générations, l'argent sert, au niveau des chefs de lignage, à maintenir leur autorité chancelante, en payant ce genre de contre-prestation aux membres du lignage placés sous leur dépendance. Les femmes ont parfaitement analysé les éléments constitutifs de la situation et sont souvent les complices de leur propre enlèvement ${ }^{2}$. Les jeunes gens en sont souvent les auteurs. Ils obligent ainsi la génération qui détient actuellement le pouvoir à satisfaire à ses obligations. De son côté, le mari trompé a une attitude extrêmement ambiguë. Si sa femme n'est pas convoitée, il pense qu'il a fait là une mauvaise affaire et, si elle est enlevée, il revendique des dommages-intérêts pour le préjudice moral qui lui est infligé. Il est vrai que Kobben voit, dans la complexité de cette situation, de nombreux éléments inhérents à la situation de jeu. Quoi qu'il en soit, ce type de relation donne à la femme bété des possibilités d'autonomie dont elle est prête à profiter.

La relation entre le montant de la contre-prestation et la répartition de l'activité au sein de la famille nucléaire, d'une part, et le climat affectif, d'autre part, fait actuellement l'objet d'un test portant sur la population abouré et bété. Les résultats ne sont pas encore connus.

Si l'introduction des cultures industrielles et de l'économie de profit a contribué à modifier le système des relations interfamiliales, il en va de même pour la résidence ${ }^{3}$ : l'individualisation de la propriété, le développement de la culture extensive, qui mène à la création de campements, l'urbanisation ont abouti à ce que Murdock appelle la règle néo-locale : le nouveau ménage a tendance à choisir une nouvelle résidence ${ }^{4}$. Cette règle est bien plus appliquée à la ville qu'à la campagne ; cependant, elle commence à supplanter la patrilocalité, qui était la règle universelle en Côte-d'Ivoire, jusqu'à présent.

Cette nouvelle règle se traduit par une augmentation de la distance qui sépare la famille nucléaire de la famille étendue. La force qu'exerce le lignage sur le mari et la femme diminue donc d'autant. Parallèlement, les rôles se différencient. Les absences du mari du foyer se font plus longues (s'il est salarié); les possibilités que rencontre la femme de s'insérer dans une économie de profit vont donc s'accompagner à la fois d'une redistribution des rôles et des règles d'autorité et d'une redistribution des recettes et des dépenses. De plus, la primauté de l'intégration économique sur l'intégration sociale amène une baisse sensible de la polygynie, qui n'est plus perçue comme signe de richesse mais comme cause de dépense. Toutes ces modifications auront des implications sur le plan des attitudes.

Enquête sur le niveau de vie et la nutrition à Bongouanou, p. 117.

Kobben, op. cit., p. 136.

Murdock, op. cit., p. 201 et suiv.

Mercier, dans : Études africaines, no 1, et dans: Aspects sociaux de l'industrialisation et de l'urbanisation en Afrique au sud du Sahara, p. 543. 
En effet, les systèmes de rôles se séparent du système de parenté ${ }^{1}$ et les changements relatifs à la famille ne sont que les reflets de changements plus profonds, touchant le système social dans son ensemble. La société de la Côte-d'Ivoire, fondée sur des principes de particularistic ascription, évolue peu à peu vers un modèle de société industrielle fondée sur le principe de l'universalistic achievement. Les statuts individuels tendent à être déterminés plutôt par des critères relationnels que par des critères de classification des qualités. La motivation est essentiellement axée sur la réussite obtenue à partir de facteurs cognitifs.

La mobilité géographique des individus augmente et leur stratification s'opère sur la base d'un système de classes sociales ouvertes, tandis que diminue l'intégration des croyances religieuses dans le système social.

Cet ensemble de traits culturels caractérise bien la société française. Il est donc de plus en plus aisé de faire une comparaison entre les attitudes adoptées à l'égard de la femme dans la société ivoirienne et dans les sociétés européennes mises en cause par l'enquête, en faisant toutefois trois réserves : a) le système de structuration sociale «à l'européenne » est encore récent ; b) il est plus global et moins différencié ; c) il s'appuie sur une culture historiquement différente.

La première dimension examinée dans l'enquête française sur les attitudes de la société à l'égard de la femme est relative à l'âge du mariage. La différence d'âge entre mari et femme constitue, en effet, un excellent indice du modèle retenu pour définir la relation inter-époux : autoritaire ou coopératif.

Sur l'échantillon expérimental de 213 familles ${ }^{2}$, il a été remarqué que l'âge moyen du mariage se situait, pour les filles, avant 18 ans et, pour les garçons, après 23 ans. Cette différence d'âge importante diminue au sein du groupe agni et il est remarquable que cette population soit à la fois la plus scolarisée et la plus représentée dans toutes les professions libérales en Côte-d'Ivoire. D'une manière générale, les filles semblent décidées à se marier jeunes et à rechercher un mari plus âgé qu'elles.

On peut interpréter ce phénomène comme traduisant, dans une large mesure, l'acceptation, par les femmes, d'un modèle autoritaire dans leurs relations avec leur mari.

Trois variables agissent en sens contraire : a) le degré d'acculturation du groupe ethnique auquel appartiennent les femmes ; b) le système de lignage en vigueur dans le groupe en cause : dans la mesure où les groupes patrilinéaires mettent en valeur une relation hiérarchique entre générations, alors que les groupes matrilinéaires insistent sur une relation plus égalitaire entre frères et sœurs, on peut se demander si le système de matrilignage ne détermine pas une différence d'âge plus faible entre époux (cette hypothèse fait présentement l'objet de tests portant sur les populations abouré et bété) ; c) le degré d'acculturation de la femme; la poursuite d'études - qui correspond à la recherche d'une certaine autonomie financière et à l'évasion hors des contraintes du milieu familial -empêche, par là même, la femme scolarisée de se marier jeune et d'accepter les prémisses d'une union autoritaire.

Talcott Parsons. Theory of action, p. 178.

Femmes ivoiriennes. Études expérimentales sur l'évolution de la famille en Côte-d'Ivoire, p. 11. 
L'enquête sur l'échantillon abouré et bété tendra également à étudier la relation existant entre la procédure de choix du conjoint et l'âge de la femme, en vue de contrôler précisément l'effet de l'âge sur le degré de liberté dont dispose la femme. Cette enquête, malheureusement, ne touche que les femmes. En ce qui concerne la technique de mariage pratiquée, une enquête par interviews systématiques portant sur 80 filles candidates à différentes fonctions dans les services sociaux indique que les intéressées désirent le mariage selon les règles du droit civil français, pour pouvoir plus facilement divorcer. On notera donc que ces filles, avant leur mariage, considèrent l'association avec l'homme comme risquée et semblent adopter une attitude guidée par un sentiment assez fort d'insécurité:

L'activité professionnelle exercée par les femmes semble être le plus souvent une activité de caractère commercial, destinée à leur assurer des revenus propres : la fonctionnarisation des femmes est encore un phénomène trop récent pour qu'on puisse étudier dans quelle mesure il est accepté et recherché par les membres de la famille d'origine et de la famille du mari.

La deuxième dimension a trait aux attitudes qu'adopte la société en ce qui concerne l'éducation des femmes et leur profession. Les informations actuellement disponibles sont peu nombreuses. On notera toutefois que, parmi les jeunes filles candidates à un poste dans les services sociaux, un quart ont dû affronter l'opposition de parents et, plus particulièrement, d'ascendants collatéraux. Il apparaît également que le choix des études a été opéré par les intéressées elles-mêmes, dans $30 \%$ des cas. Les progrès en matière de scolarisation impliquent une opposition assez vive entre générations - la génération parentale ne possédant pas souvent tous les éléments d'information souhaitables pour prendre une décision dans ce domaine. Cette opposition est surtout perceptible dans les milieux ruraux.

Dans le cadre de l'enquête portant sur la population bété et abouré, deux questions ont été posées dans ce domaine : la première consistait à savoir si les filles doivent recevoir la même éducation que les garçons. Il semble qu'une majorité écrasante des femmes interviewées acceptent l'hypothèse d'une égalité d'éducation. Cependant l'opinion de leur mari sur ce sujet n'est connue qu'à travers leur propre perception. Toutefois, il est intéressant de noter qu'interrogés sur ce problème en 1959, les élèves ivoiriens de la classe de cinquième du Collège d'orientation d'Abidjan ont adopté une attitude nettement moins favorable à l'égalité des sexes en matière d'éducation que leurs homologues métropolitains. Dans leur ensemble, les garçons étaient moins favorables à cette thèse que les filles. Mais, inversement, il faut noter que, dans l'échantillon expérimental, les maris les plus occidentalisés choisissent leur épouse en fonction de son niveau d'instruction : $44 \%$ des femmes de fonctionnaires savent lire et écrire, contre $5 \%$ seulement des femmes de paysans. Il reste, bien sûr, à savoir si cette modification correspond, chez l'homme, à la recherche d'un prestige extérieur ou si elle traduit une inquiétude devant les tâches qui incombent à un ménage occidentalisé - inquiétude supposant une redistribution de la division du travail et, par conséquent, de l'autorité. La jeune fille instruite semble pourtant occuper une position ambiguë auprès des hommes : si on la considère avec une certaine admiration, on la redoute aussi, comme étant difficile à diriger.

La deuxième question posée était relative à la spécialisation de l'éducation en fonction du sexe du parent et de l'enfant («La femme s'occupe-t-elle des filles et l'homme des garçons ? »). Une telle spécialisation impliquerait certainement un cloisonnement et un retrait des filles de la vie sociale et serait sans doute préjudiciable 
à une égalisation des rôles. L'analyse des réponses à cette question, en fonction de l'origine ethnique, de la résidence, du nombre d'enfants et de la catégorie socioprofessionnelle, est actuellement en cours.

Peu d'informations sont encore disponibles sur les attitudes à l'égard du travail de la femme. Toutes les jeunes filles qui étudient pour occuper un poste dans les services sociaux comptent continuer à travailler après leur mariage. Les raisons invoquées sont, dans $47 \%$ des cas, purement économiques et, dans $22 \%$ des cas, à la fois économiques et culturelles. Pour les intéressées, il s'agit, avant tout, de sauvegarder leur indépendance, dans le cadre de l'opposition entre sexes si bien analysée par Binet.

L'analyse plus poussée du choix d'un métier et des motivations sous-jacentes demeure à faire.

La troisième dimension a trait à la distribution du pouvoir dans la famille; en ce qui concerne le pouvoir financier, il est exercé, dans la moitié des cas étudiés dans l'échantillon expérimental, par le mari seul. Dans le tiers des cas, il est exercé séparément par chacun des deux époux, selon les recettes dont il dispose. Ces proportions sont identiques dans les familles des élèves ayant choisi une carrière sociale. En outre, le pouvoir budgétaire détermine les règles de gestion budgétaire. C'est ainsi que les investissements familiaux de quelque importance (vêtements) sont toujours assurés par le mari, s'il exerce le pouvoir financier. L'enquête sur la population bété et abouré doit permettre de mesurer avec plus de précision toutes les attitudes de la femme à l'égard de ce problème : cette enquête est d'ailleurs plus poussée puisqu'elle contient des questions relatives à l'achat du matériel de cuisine, à l'achat d'une maison, etc.

Le pouvoir en matière d'éducation est exercé, dans plus de la moitié des cas, par le mari. Toutefois, la participation de la femme à ce type de décision dépend de son niveau de culture.

On peut donc constater que, dans l'ensemble, le mari est la figure d'autorité dominante dans la société ivoirienne actuelle (54\% des cas). Cette position privilégiée du mari se retrouve à un niveau sentimental. Les femmes sont très tolérantes à l'égard des sorties du mari. Elles accompagnent une telle tolérance d'une attitude fataliste à l'égard d'éventuelles incartades conjugales. Cette double caractéristique se traduit par le fait que le tiers des femmes interrogées pensent que leur propre infidélité est plus grave que celle de leur mari.

Les femmes semblent donc ne pas se sentir en position de demander des comptes à leur époux, car elles subiraient des sanctions contre lesquelles elles ne pourraient rien faire (divorce, réprimandes du lignage). Toutefois, on notera que, là encore, la position des femmes de fonctionnaires et des femmes instruites est plus forte et que leur attitude est moins tolérante et plus exigeante à l'égard du mari. L'attitude des étudiantes qui se destinent aux services sociaux est caractéristique à cet égard, puisque $48 \%$ d'entre elles estiment que l'infidélité est aussi grave dans un cas que dans l'autre.

En conclusion, il semble donc que la femme, en Côte-d'Ivoire, tente d'améliorer son statut social en se mariant à un âge plus avancé (ce qui lui permet de résister plus efficacement aux sollicitations de son groupe d'origine) et en accroissant ses possibilités de s'assurer un revenu économique indépendant, soit en acquérant une 
meilleure éducation (il est significatif que les études aient pour conséquence de soustraire légalement le sujet aux rites et aux fêtes de son groupe d'origine) ${ }^{1}$, soit en exerçant une activité économique autonome (artisanat, commerce).

Cette amélioration se traduit par un nouveau partage de l'autorité et des tâches familiales. Ayant pour effet de diminuer la cohésion du groupe, l'urbanisation éloigne souvent le mari de son foyer, pour de nombreuses raisons, tant économiques que sociales et affectives. La femme et les enfants demeurent alors les seuls éléments stables de la famille ${ }^{2}$. L'acculturation de la femme semble donc avoir pour effet éventuel, compte tenu de l'opposition déjà analysée entre les sexes, d'augmenter son pouvoir et ses tâches dans une perspective séparatiste. Les pouvoirs des époux, relativement égaux quantitativement, sont alors exercés sans aucune collaboration. Il arrive aussi que la femme devienne la figure d'autorité dominante du ménage. Cette tendance apparaît déjà timidement à Treichville. C'est, semble-t-il, une constante du monde noir, puisque Binet, à propos des Antilles, et de nombreux sociologues, aux États-Unis, ont fait des remarques analogues. La tendance unitaire de la société européenne se heurte ici à une réaction de double opposition : entre les lignages et entre les sexes. Il apparaît donc normal que l'attitude à l'égard des femmes évolue dans des directions différentes.

Nous avons donné un aperçu du problème posé par l'attitude des femmes envers leur statut au sein de la société ivoirienne. L'enquête sur les populations bété et abouré doit permettre de préciser de nombreux points et d'obtenir des informations plus cohérentes.

En ce qui concerne l'analyse de l'attitude de l'homme vis-à-vis du même problème, deux enquêtes sont actuellement en préparation :

a) Une enquête auprès des hommes bété de la région de Gagnoa, dont les femmes ont été interrogées. Cette enquête a un double objet : elle doit permettre, d'abord, de déterminer si le statut attribué à chaque sexe infléchit son système de perceptions relatif à la vie familiale ; elle doit permettre, ensuite, de déterminer ce que les hommes attendent de leurs relations avec leurs femmes.

b) Une deuxième enquête, qui doit porter sur une population d'employés de commerce. Elle analysera surtout les attitudes de l'homme à l'égard du travail féminin (vers quels métiers la femme doit-elle être orientée et en fonction de quels critères ?). Ces attitudes seront analysées en corrélation avec les attitudes touchant la répartition des pouvoirs à l'intérieur de la famille.

Ainsi sera étudiée la motivation de cette catégorie socio-professionnelle à l'égard du rôle à attribuer aux femmes dans la société globale.

Le rapport d'ensemble sera publié ultérieurement. Les variables indépendantes étant l'âge, le sexe, le niveau culturel, la profession et la résidence, les sujets analysés seront - le mariage (procédure et âge), les problèmes posés par le travail des femmes et les relations entre époux (autorité, climat affectif).

1 La jeune fille adioukrou qui a reçu une certaine instruction est systématiquement remplacée, dans les fêtes qui intéressent son groupe d'âge, par une parente illettrée, du même âge ou plus jeune.

2 Binet, op. cit., p. 750. 
IV

Trois études préliminaires: Canada, Côte d'Ivoire, Togo

\section{III}

\section{"Recherche sur les attitudes vis-à-vis de la femme togolaise" \\ F. N'Sougan Agblemagnon}

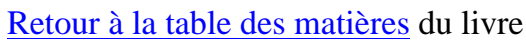

Table des matières de la 3e analyse préliminaire au Togo

1. La personnalité sociale de la femme africaine
a) La femme dans les circuits matrimoniaux
b) La situation juridique de l'épouse
c) Le rôle de la femme dans le circuit économique
d) Les attitudes vis-à-vis de l'éducation de la jeune fille
e) La femme dans le système politique et le circuit des alliances de la communauté
f) La femme et le système des rituels

2. La situation de la femme dans la société éwé

3. Comment la femme est-elle perçue dans les sociétés africaines traditionnelles?
a) Quelles sont les attitudes qui découlent de cette perception (pour les hommes) ou
qui la renforcent ou la justifient (pour les femmes elles-mêmes) ?
Perturbation mais non suppression ou disparition de cette perception et des attitudes traditionnelles

4. Suggestions en vue d'une enquête éventuelle sur les attitudes vis-à-vis de la femme dans une société africaine : le cas des éwé du Sud-Togo
a) Comment la femme perçoit-elle le monde féminin ?
b) Comment l'homme perçoit-il le monde féminin? 
$\underline{\text { Retour à la table des matières de l'article sur les femmes togolaises }}$

En 1958, du 15 au 25 juillet, s'est tenue à Lomé, capitale du Togo, une importante réunion féminine, groupant « 300 représentantes de 10 États de l'ouest de l'Afrique Cameroun, Congo belge, Congo français, Côte-d'Ivoire, Dahomey, Ghana, Guinée, Haute-Volta, Nigeria, Togo »- avec la participation de « Mlle du Rostu, présidente de l'Union mondiale des organisations féminines catholiques, qui avait pris l'initiative de ces journées ». Pour la première fois, solennellement, les femmes africaines démontraient que non seulement elles pouvaient penser, mais qu'elles pensaient leurs propres problèmes. En effet, comme le dit la première phrase du résumé de ces journées : «La femme africaine est sur la sellette ${ }^{1}$. » Mais nous devons avouer que ses problèmes n'ont pas toujours été abordés avec la clarté qui s'impose. jusqu'ici, la femme africaine a surtout été perçue dans un circuit d'échanges; quelquefois, on s'est plu à la penser à travers une philosophie égalitaire, en se demandant dans quelles conditions elle était ou n'était pas l'égale de l'homme, pris abstraitement d'ailleurs; on s'est évertué à montrer sa position dans le ménage, que ce soit par rapport au problème de la polygamie ou par rapport à celui de la dot; mais nous devons avouer que ces théories sur la femme africaine sont, le plus souvent, des opinions d'hommes et d'ethnologues évoquant des situations précises, intéressantes, mais qu'en règle générale elles font peu de cas des attitudes et, en premier lieu, des attitudes des femmes elles-mêmes.

Or il nous semble que, tant pour une sociologie de la famille que pour une sociologie de la connaissance, il est plus que jamais indispensable d'entreprendre une étude sur les attitudes de la femme africaine. En effet, cette étude, pour la première fois, pourra faire intervenir activement les femmes dans l'enquête, au lieu de leur réserver un rôle passif, soit en les décrivant dans les structures sociales, soit en récapitulant ce que les hommes pensent d'elles. Il faut d'ailleurs avouer aussi que, dans beaucoup de cas, les travaux de certains ethnologues sur la femme africaine n'ont fait que justifier les préjugés que ces mêmes ethnologues avaient sur la femme de leur propre société. Dans les lignes qui vont suivre, l'unique question que nous nous posons est la suivante - Comment peut-on amorcer une étude sur les attitudes vis-à-vis de la femme en Afrique noire, qu'il s'agisse des attitudes de la société traditionnelle ou des attitudes à l'égard de la femme dans la situation existant actuellement. Dans une autre partie, nous envisagerons - ou plutôt nous poserons - la question de savoir comment, concrètement, pourrait se dérouler une enquête sur les attitudes et les perceptions dans un pays africain comme le Togo.

1 Femmes africaines, Paris, Édition, du Centurion, 1959, p. 4. 


\section{La personnalité sociale de la femme africaine}

Tout d'abord, nous allons éviter autant que possible les généralités et une certaine confusion qui consiste à ne pas dissocier ce dont on veut parler de ce dont on veut entreprendre la recherche. En effet, le problème de la femme est un des plus vastes : S'agit-il de la condition humaine de la femme dans les sociétés africaines ? S'agit-il de l'apprentissage du métier de femme dans ces sociétés ? S'agit-il des étapes de la vie de la femme africaine ? S'agit-il des problèmes spécifiques de la fille, de l'épouse, de la mère, de la citoyenne ? S'agit-il des processus d'inclusion de la femme dans les sociétés africaines, à la fois dans son propre lignage, dans le lignage de son mari, dans la société globale, dans les associations spécialisées ? S'agit-il de la femme au travail ? S'agit-il de la femme et du mariage ? S'agit-il de la femme et de l'éducation ou plutôt de l'éducation de la femme ? S'agit-il du statut de la femme dans les diverses structures africaines ? Ou encore, pour terminer, s'agit-il des rythmes de la vie de la femme, que ce soit sur le plan biologique, sur le plan social ou sur le plan professionnel ? On voit qu'il importe, pour l'étude de cette personnalité sociale de la femme africaine, de nous limiter volontairement à quelques points précis.

\section{a) La femme dans les circuits matrimoniaux}

$\underline{\text { Retour à la table des matières de l'article sur les femmes togolaises }}$

Il est incontestable que, dans toutes les sociétés africaines, la femme occupe un statut privilégié et joue un rôle prépondérant dans les circuits matrimoniaux, que ce soit parce que sa propre personne est l'enjeu de ces relations matrimoniales ou que ce soit par son pouvoir d'intervention dans la conclusion des accords matrimoniaux, en tant que fille donnant ou ne donnant pas son consentement, en tant que mère, tante ou parente, donnant ou ne donnant pas son approbation.

\section{b) La situation juridique de l'épouse}

Beaucoup d'études montrent que, dans les sociétés africaines, la femme, tout en entrant dans la famille de son mari, continue d'appartenir à sa propre famille - et ceci avec les avantages et les désavantages qui s'y attachent. Désavantages : elle se sent toujours étrangère dans le groupe de la société du mari, dans le lignage du mari, où elle ne s'inclut que provisoirement; avantages : au moment des crises, elle aura 
toujours l'ultime recours de retourner dans sa propre famille, où elle traite, en quelque sorte, les affaires les plus importantes.

\section{c) Le rôle de la femme dans le circuit économique}

Bien sûr, les prestations qui sont provoquées ou qui entrent en jeu à l'occasion de l'établissement ou du fonctionnement des circuits matrimoniaux concourent déjà à situer la femme, malgré elle, dans un premier circuit économique, mais il y a aussi un autre aspect du problème - volontaire celui-là : Comment la femme s'inclut-elle ellemême dans les circuits économiques ? Dans la plupart des sociétés africaines, on sait qu'elle possède, en propre, ses gains, qu'il s'agisse de ce que son mari lui donne ou des fruits de l'élevage ou du petit commerce auquel elle se livre - et que sa situation est celle d'une personne indépendante.

\section{d) Les attitudes vis-à-vis de l'éducation de la jeune fille}

Ce problème est très important. Dans la société traditionnelle, nous voyons partout un système qui réglemente rigoureusement l'éducation de la jeune fille. Nous savons que, dans beaucoup de sociétés africaines, l'éducation de la fille incombe, dans la majorité des cas, à la mère, alors que celle du garçon incombe au père. Cette scission de deux mondes - un monde féminin et un monde masculin - à partir, précisément, de la première enfance peut avoir des résonances sur la manière dont les deux groupes, masculin et féminin, vont s'opposer dans la vie concrète. Y a-t-il là un modèle d'opposition des sexes ? Cette opposition, en tout cas, ne peut pas être affirmée ; il existe, sur ce plan, un rapport de complémentarité plutôt que d'opposition. Le problème moderne concerne la manière dont la jeune fille doit avoir accès à la société moderne et il s'agit de savoir si la société africaine veut la favoriser ou la freiner dans son élan.

\section{e) La femme dans le système politique et le circuit des alliances de la communauté}

\section{$\underline{\text { Retour à la table des matières de l'article sur les femmes togolaises }}$}

La femme africaine, en entrant dans un circuit matrimonial, établit des rapports entre deux communautés plutôt qu'entre deux individus. À ce titre, elle n'est qu'une sorte de monnaie des alliances et de la bonne entente entre des groupes et, en quelque sorte, une espèce de «monnaie diplomatique » précieuse, maintenant des équilibres politiques précaires; mais nous savons aussi qu'elle peut, par son intelligence et par sa personnalité, jouer un grand rôle au sein de sa propre communauté, si ce n'est entre plusieurs communautés. Bien que les situations de la femme africaine soient très diverses, on peut, d'une manière générale, dire que les systèmes sociaux africains ont fait à la femme une place que souvent on n'a pas suffisamment mise en lumière. 


\section{f) La femme et le système des rituels}

Au niveau des mythes, au niveau de la pratique rituelle, et même de la conception du monde, de la philosophie cosmique, la femme entre dans le système religieux, et son rôle (l'image de la vieille femme, de la sorcière) peut être important dans beaucoup de cas. Par conséquent, ni le système économique, ni le système politique, ni le système du lignage, ni le système religieux ne mettent la femme à l'écart. Finalement, nous percevons, au niveau des consciences africaines et à travers la littérature orale africaine une image idéalisée de la femme sous l'aspect de la mère. L'importance de l'idée de la mère a d'ailleurs été fort bien soulignée par divers auteurs (voir notamment le livre qui vient d'être publié par D. Paulme ${ }^{1}$.

Prenons comme exemple la situation de la femme dans une société africaine du sud du Togo, la société éwé.

\section{La situation de la femme dans la société éwé}

$\underline{\text { Retour à la table des matières de l'article sur les femmes togolaises }}$

Cette société patrilinéaire et patrilocale repose sur une complémentarité assez heureuse des sexes. On peut dire que la femme n'y est jamais frustrée à cause de son sexe. Elle n'a pas eu à combattre pour assurer sa place dans le système traditionnel; plusieurs dispositions juridiques attestent la volonté de cette société d'inclure la femme en tant qu'élément très important du système social. Cette place est attestée par l'importance spécifique reconnue à chaque sexe dans l'équilibre social (division harmonieuse du travail). Le principe démocratique, élément essentiel de cette société, s'applique non seulement dans les relations entre hommes, mais dans les relations entre hommes et femmes. Dans le mariage, la femme, en allant vivre dans la famille de son mari, ne cesse pas, pour autant, d'appartenir à son propre lignage. Ce groupe d'appartenance est aussi le groupe où elle trouvera immédiatement refuge lorsqu'elle quittera la maison de son mari (pour une raison ou pour une autre), notamment si celui-ci la maltraite. Elle peut pousser sa protestation jusqu'au divorce et personne, même le chef, ne peut l'obliger à réintégrer contre son gré le domicile de son ancien mari. Il n'y a pas, à proprement parler, de rouages sociaux capables de forcer sa volonté et de lui imposer ce pourrait juger contraire à ses intérêts et à ses sentiments.

1 D. Paulme. Femmes d'Afrique noire. Éd. Mouton, Paris et La Haye, 1960, 282 p. 
Elle aide son mari aux champs, puisque l'activité traditionnelle est surtout agricole. Dès la fin du siècle dernier, J. Spieth était fortement impressionné par l'image du père, de la femme et des enfants travaillant ensemble aux champs ${ }^{1}$ : la famille éwé est une communauté et cette communauté est aussi un groupe de travail.

La femme peut d'ailleurs cultiver, pour son propre compte, un lopin de terre dont elle vend les produits et conserve les fruits. Elle peut augmenter ce pécule, soit par le commerce ${ }^{2}$ de menus objets sur la place publique du village ou dans la rue de la ville, soit par un véritable commerce plus ou moins important ${ }^{3}$.

Homme et femme concourent aux frais du mariage. Dans les ménages paysans, le mari fournit généralement l'igname, le maïs et la viande, tandis que la femme constitue la provision de piment, de sel et achète le poisson au marché.

Dans le Sud, l'évolution moderne a souvent entraîné un curieux renversement des rôles. Il s'est, en effet, constitué un groupe puissant de « revendeuses », affirmant un type nouveau de femmes, qui non seulement ont plus de moyens matériels que leur mari, mais manifestent à tous égards leur indépendance. Nous avons pu prendre conscience de la manière dont ce groupe conçoit l'évolution de la femme et de l'Afrique, grâce à l'interview que nous a accordée la présidente du syndicat de ces revendeuses. Nous avons aussi pu observer concrètement la manière dont cette indépendance de la femme affecte la vie des ménages. Ainsi la femme du « commis », qui attend « l'argent du mois » de son mari, est plus dépendante de celui-ci que la revendeuse qui non seulement peut se passer de cet argent, mais peut aider son mari à sortir d'un embarras financier passager. D'une manière générale, cette indépendance de la femme est entièrement tolérée par le mari : l'intéressée peut se rendre à un marché lointain, s'absenter toute une journée ou même plusieurs jours.

Dans la vie politique, la femme manifeste une personnalité distincte, indépendante de celle de l'homme et de celle du mari. Il existe de puissantes organisations féminines, dont les tâches sont multiples et souvent politiques. Et c'est en tant que citoyenne consciente que la femme fait partie de ces diverses associations. Il semble même que la société traditionnelle ait prévu, d'une manière plus rigoureuse encore, la place et le rôle de la femme. Souvent autrefois, à côté du chef de village, une femmechef était élue. Cette dernière avait droit aux mêmes honneurs que le chef homme. Elle traitait, en priorité et en premier ressort, toutes les questions concernant des femmes. Les attributions de cette femme-chef pouvaient souvent aller très loin. Si normalement, en temps de paix, le rôle de la femme en tant que citoyenne consistait surtout à maintenir la propreté, à veiller au respect du code moral et à l'éducation des enfants (surtout des filles), en temps de guerre, ce rôle était souvent accru. Certes, la femme restait souvent à la maison, en compagnie des vieillards et des enfants, sous la responsabilité d'un personnage désigné à cet effet, pendant que les guerriers étaient au combat. Pour l'essentiel, les femmes remplaçaient les hommes absents. Elles n'étaient pas seulement les gardiennes de la maison, mais constituaient un groupe ayant pour tâche à veiller au moral des guerriers. Si un fuyard revenait discrètement à la maison, un groupe de femmes se chargeait aussitôt de le ramener dans le droit chemin. Si le déserteur tardait à s'exécuter, il était hué, menacé et même souvent mis à mort. Un certain nombre de femmes accompagnaient les guerriers au combat. Leur rôle

Jakob Spieth. Die Ewe Stämme, Berlin, 1906.

Agbonusi ou «marché du pas de la porte».

Asitsatsa ou «pratique qui consiste à parcourir les marchés ». 
consistait à remplir les tâches relevant de l'intendance, à faire la cuisine, à s'occuper des blessés et des morts. On voit, par ces quelques exemples, que la femme, à tous les échelons, s'intégrait parfaitement dans la vie de la communauté éwé.

Ces caractéristiques de la société traditionnelle, jointes aux manifestations d'initiative dont font Preuve, dans le domaine économique, dans le domaine politique et dans le domaine social, les femmes du sud du Togo, militent puissamment en faveur d'une enquête sur les attitudes vis-à-vis de la femme dans cette partie de l'Afrique.

\section{Comment la femme est-elle perçue dans les sociétés africaines traditionnelles?}

$\underline{\text { Retour à la table des matières de l'article sur les femmes togolaises }}$

Tout ce que nous venons de dire - à savoir que la femme était perçue sous les traits complémentaires et contradictoires que nous venons d'énumérer - reste encore valable ; mais la femme était, avant tout, perçue par les sociétés africaines comme épouse. En effet, la petite fille pouvait être, on le sait, fiancée dès le berceau ; l'image que les hommes s'en faisaient n'était pas seulement celle d'une petite fille, mais celle de la future épouse de quelqu'un ; on la préparait à ce rôle d'épouse ; l'image que sa communauté paternelle en avait était celle d'une personne qui, tôt ou tard, quitterait le lignage, mais pour renforcer, d'une certaine manière, des alliances avec d'autres lignages ou d'autres groupes; et ce que la société en attendait, c'étaient les devoirs de la bonne épouse, c'est-à-dire de la bonne mère qui donnerait beaucoup d'enfants à la communauté ; pour le mari, la question était de savoir dans quelle mesure elle pourrait contribuer à accroître le nombre de ses enfants (prestige) et, le cas échéant, sa richesse. Par conséquent, dans les sociétés africaines traditionnelles, pour des raisons différentes, la femme est d'abord perçue comme épouse ; c'est pourquoi, en Afrique, l'accent primordial est mis sur la vertu par excellence de l'épouse, à savoir la fécondité.

Or ce à quoi nous assistons par la suite, c'est l'altération de ce qui apparaissait comme le plus important des critères de la vision de la femme dans les sociétés africaines. 


\section{a) Quelles sont les attitudes qui découlent de cette perception (pour les hommes) ou qui la renforcent ou la justifient (pour les femmes elles-mêmes)?} mêmes.

Il faut considérer : a) les attitudes des hommes; b) les attitudes des femmes elles-

Les attitudes des hommes. Elles sont connues. C'est l'attitude du père envers sa fille (façon dont il accepte la naissance d'une fille), l'attitude du mari vis-à-vis de sa femme, l'attitude de la famille de la jeune femme à son égard, l'attitude de la famille du mari vis-à-vis de la jeune femme et l'attitude globale de la société vis-à-vis de la femme en tant que telle (place qui lui est faite dans le système religieux, dans le système économique et dans le système politique).

Les attitudes des femmes. Il s'agit, tout d'abord, de ce que les femmes pensent de leur propre condition (telle qu'elle est ou telle qu'elle a été dans les sociétés traditionnelles) et, deuxièmement, des critiques qu'elles adressent à l'ancien système ou des suggestions qu'elles font pour améliorer leur situation actuelle. D'une manière générale, on peut dire qu'actuellement il y a un problème de la femme, du fait de la transformation générale des systèmes sociaux et de la transformation de la mentalité féminine.

\section{b) Perturbation mais non suppression ou disparition de cette Perception et des attitudes traditionnelles}

$\underline{\text { Retour à la table des matières de l'article sur les femmes togolaises }}$

La situation coloniale a ignoré presque totalement la femme; celle-ci a subi une régression sur beaucoup de points, dans la mesure où sa situation sociale dépendait de l'ancien ordre de choses, des structures traditionnelles. Même aujourd'hui, dans une certaine mesure, les femmes sont plus conservatrices que les hommes. Est-ce parce qu'elles sont moins touchées par l'éducation moderne ? En tout cas, c'est une question qui reste posée.

La femme ressentira plus fortement que l'homme les changements qui ont des répercussions directes sur sa vie de jeune fille, d'épouse, de travailleuse, de citoyenne, etc.

Elle acquiert une nouvelle mentalité par rapport à elle-même et par rapport à sa situation - ce qui se traduit souvent par la naissance d'un complexe d'infériorité, de domination ou de frustration et par l'apparition de la conscience d'un retard à rattraper; elle est ainsi amenée à s'en prendre soit au colonisateur, soit à l'homme, soit à la société traditionnelle. 
Mais, en même temps, la condition de la femme s'est différenciée. On peut distinguer plusieurs catégories de femmes : la femme illettrée de la campagne (paysanne traditionnelle) ; la femme instruite de la campagne; la jeune fille illettrée de la campagne; la jeune fille instruite de la campagne; la jeune fille citadine illettrée; la jeune fille citadine instruite; la jeune femme citadine instruite mais n'ayant reçu aucune formation professionnelle; la jeune femme citadine instruite et ayant reçu une formation professionnelle (dactylo, sténodactylo, secrétaire, infirmière, etc); la jeune femme appartenant aux cadres moyens; la jeune femme appartenant aux professions libérales (professeur, etc.); la commerçante aisée (homologue du planteur masculin).

Outre l'éducation et la profession, d'autres facteurs interviennent dans ce processus de différenciation de la condition féminine : le poids de la culture locale, le système religieux, la structure du ménage (polygamie, monogamie, etc.), les attitudes des pouvoirs publics et le rôle des organisations féminines, ainsi que des mouvements paraféministes. On peut dire que les attitudes vis-à-vis de la femme se manifestent spontanément à propos des points suivants : éducation des femmes, relations de la femme et du mari, structure du ménage et image du ménage idéal, égalité des sexes, travail de la femme (ceci est valable pour certaines catégories seulement, l'expression étant prise dans son acception occidentale), prérogatives de la citoyenne.

\section{Suggestions en vue d'une enquête éventuelle sur les attitudes vis-à-vis de la femme dans une société africaine : le cas des éwé du Sud-Togo}

$\underline{\text { Retour à la table des matières de l'article sur les femmes togolaises }}$

Remarquons d'abord que, pour des raisons tenant à l'état actuel des structures sociales africaines, il serait souhaitable que cette étude tende à donner une vue globale du problème. Nous suggérons donc qu'elle porte sur les points suivants :

\section{a) Comment la femme perçoit-elle le monde féminin ?}

À travers quel système de modèles - africain ou européen - telle ou telle femme perçoit-elle la femme?

Que pense chaque femme des femmes de sa propre catégorie et des femmes des autres catégories ? (L'objet de cette question est de faire apparaître les critiques, les préjugés, les stéréotypes féminins.) Que pense la femme des hommes, en général, et des hommes de son milieu en particulier? 
Comment les femmes jugent-elles la situation actuelle de la femme?

Que souhaitent-elles : sur le plan du mariage, sur le plan du travail, sur le plan politique, etc. ?

\section{b) Comment l'homme perçoit-il le monde féminin ?}

\section{$\underline{\text { Retour à la table des matières de l'article sur les femmes togolaises }}$}

Il est indispensable de recouper les attitudes féminines par les attitudes masculines, car, dans la société globale, réelle, c'est de la rencontre ou de l'interférence permanente de ces deux catégories d'attitudes que résulte la situation concrète de la femme. Il s'agit de saisir, en quelque sorte, l'image de la femme modèle dans la société actuelle (compte tenu du fait qu'à la vision du présent se mêlent intimement les aspirations d'avenir). Cette image doit être saisie sur le plan domestique, sur le plan conjugal, sur le plan économique, sur le plan politique, sur le plan de l'éducation et sur celui de la hiérarchie sociale.

À ces deux points, il conviendrait d'ajouter : une étude des groupements féminins modernes et des mouvements féminins (syndicats, associations de revendeuses, Union des femmes togolaises, chorales féminines, sections féminines des partis politiques, etc.); et une étude de biographies de femmes types (par exemple : la revendeuse, la femme exerçant des fonctions de dirigeante moderne (notamment de syndicat), la paysanne traditionnelle, la jeune fille illettrée des villes, la jeune femme employée comme manœuvre à la ville (Lomé), la jeune dactylo, la jeune infirmière, l'institutrice, la couturière, la conseillère municipale, etc.).

Ces biographies ou autobiographies auraient pour objet de fournir des éléments complémentaires qu'une enquête, forcément rapide, ne pourrait dégager suffisamment.

C'est à partir de l'ensemble des suggestions ainsi faites et du contexte culturel africain que l'on pourra évidemment élaborer le questionnaire destiné à cette étude. 


\title{
V \\ La femme autrichienne son rôle et l'image qu'on s'en fait
}

\author{
par Léopold Rosenmayr
}

Retour à la table des matières du livre

Table des matières de l'article sur la femme autrichienne

1. Conflits entre les taches fonctionnelles pratiques et les images résultant des traditions culturelles

2. Données relatives à la femme qui travaille et a la mère en ce qui concerne l'Autriche

3. Propositions et hypothèses en vue de nouvelles recherches

\section{Conflits entre les taches fonctionnelles pratiques et les images résultant des traditions culturelles}

$\underline{\text { Retour à la table des matières de la femme autrichienne }}$

Dans les discussions générales ainsi que dans les recherches sociologiques, on s'est préoccupé plus souvent du rôle de la femme que de celui de l'homme, peut-être parce que toute étude sur les activités extrafamiliales de la femme - en particulier sur le travail féminin - soulève immédiatement la question de savoir comment la femme qui travaille s'acquitte des tâches qui lui incombent dans son ménage et au sein de sa famille. L'étude du rôle de la femme a porté principalement sur les difficultés et les 
conflits qui résultent essentiellement de la double fonction d'épouse et de mère qui travaille.

Dans quelle mesure le mari ou le père est-il empêché, par l'exercice de sa profession, de remplir ses obligations familiales ? Personne n'a encore étudié la question, parce qu'on admet plus volontiers la situation «marginale » de l'homme dans la famille. Peut-être y a-t-il à cela deux raisons : d'une part, le mari s'occupe, en principe, beaucoup moins que la femme des soins du ménage; d'autre part, bien qu'on le reconnaisse moins généralement et moins franchement, on considère qu'il n'exerce qu'une influence secondaire sur le bien-être et l'éducation des enfants.

En outre, ce n'est que depuis quelques dizaines d'années que les femmes participent directement à la vie collective, et non plus par l'intermédiaire de leur mari, qu'elles jouissent de l'indépendance juridique et politique et qu'elles s'intègrent dans la production et l'économie extrafamiliale ; ce sont des faits qui ne manquent pas de provoquer quelque tension. La conciliation entre le rôle du mari et celui de l'homme appelé à travailler loin de sa famille s'est opérée plus tôt et avec moins de difficultés cette évolution étant étroitement liée à l'industrialisation et à l'urbanisation ${ }^{1}$. Si, dans les premières phases de l'industrialisation, il n'était pas rare que des femmes des classes inférieures travaillent en usine, ce n'est qu'au moment où l'industrie qui s'était créée en Europe occidentale et centrale et aux États-Unis d'Amérique dans la seconde moitié du XXe siècle s'est largement développée et étendue que ses besoins en maind'œuvre ont eu pour effet d'attirer les femmes des classes moyennes et même des classes supérieures de la société. Alors que l'homme marié et père de famille a répondu à l'appel de l'industrie il y a une centaine d'années et qu'il est devenu le « gagne-pain » séparé de la famille, l'emploi des femmes dans la production industrielle, dans l'administration et dans le système de distribution engendre, à notre époque, de nombreuses difficultés. Celles-ci tiennent probablement à deux causes. Tout d'abord, la société est devenue plus sensibles aux fâcheux effets psychiques et fonctionnels qui peuvent résulter des conflits provoqués par le double rôle de la femme, partagée entre son travail hors du foyer et ses obligations familiales. En second lieu, la séparation entre le foyer et le lieu de travail, qui est devenue «naturelle » pour l'homme, ne peut aller au-delà d'un certain point pour la femme, sans quoi il lui serait tout à fait impossible de remplir au foyer les fonctions qui créent les liens entre les différents membres de la famille.

Les conflits qui surgissent entre les fonctions de la mère et celles de la femme qui travaille ne sont d'ailleurs pas dus aux seules difficultés pratiques inhérentes au partage du temps et des forces de l'intéressée entre des activités différentes et souvent divergentes. La littérature et les arts de l'Europe ont glorifié la femme, en qui l'on voyait la gracieuse compagne que l'homme cherchait à conquérir et à laquelle il pouvait prétendre si sa situation économique et sociale était satisfaisante ; après le mariage, la femme était considérée comme le centre et l'animatrice du foyer; parée de toutes les vertus de l'épouse fidèle, elle savait concilier harmonieusement toutes les nécessités pratiques du «foyer» et de la famille ${ }^{2}$. Ces deux aspects de la femme, avant et après le mariage, se manifestaient dans le domaine de la vie privée. Dans l'histoire sociale de l'Europe, le fait pour l'homme de se marier, de conquérir une

Voir R. Stadehnann et W. Fischer, Die Bildungswelt des deuischen Handwerkers um 1800, Berlin, 1955 ; et L. Rosenmayr, Wohnen in Wien, Wien, 1956.

2 Voir Otto Brunner, «Das ganze Haus und die alteuropäische Okonornik »Neue Wege der Sozialgeschichte, Göttingen, 1956; et G. Weippert, Das Dorf in der industriellen Entwicklung der Gegenwart, Wiener Studien zur Agrarpolitik und Agrarsoziologie, Band 1, Göttingen, 1957. 
femme et de s'en assurer la possession était lié à des idées de loisirs et de distractions, à l'espérance de l'intimité hors du travail. Nous savons, il est vrai, que, même au moyen âge ${ }^{1}$, les femmes accomplissaient certains travaux hors du foyer et que, dans la société préindustrielle, une part des travaux agricoles revenait également aux femmes. Cependant, dans la société ancienne, les principaux attraits que présentait la femme pour les paysans, malgré tout le réalisme de ceux-ci, ne différaient guère des jugements de valeur et des idéaux de la classe urbaine, moyenne ou supérieure, où ce que l'homme demandait à la femme était qu'elle répondît à son désir de beauté et de plaisir, et à son besoin d'ordre et de repos après le travail. On voit donc combien le mode de vie qui résulte de l'intégration de la femme dans le mécanisme de la production et des opérations industrielles s'écarte de la conception idéale du rôle de la femme, telle qu'elle s'exprimait dans les arts et la littérature, et telle qu'elle était transmise par les traditions sociales et même par les éthiques. Les obligations qu'impliquent les fonctions de mère et de travailleuse contrastent violemment avec l'image de la femme qui avait été, de tout temps, chère au cœur de l'homme. Pour étudier les tensions psychologiques et sociologiques qui résultent du rôle de la femme, il faut examiner non seulement l'effort qu'exigent un trop grand nombre d'obligations, mais aussi les différences qui existent entre la réalité nouvelle de l'emploi en masse des femmes hors du foyer et l'image traditionnelle que l'on se faisait de la femme. Outre la description des conditions sociales, des tâches et du comportement, le sociologue devra donc étudier «l'image de la femme et de son rôle », telle que la conçoivent les différents groupes de la société.

L'étude des «images directrices » (Leitbilder) des valeurs qui déterminent le rôle de la femme se trouvera certainement facilitée si l'on commence par décrire brièvement ce rôle, tel qu'il se présente et qu'il est tenu, en fait, dans une certaine société. Nous exposerons rapidement ensuite la façon dont il conviendrait d'étudier les images de ce rôle. De cette première partie - analyse du rôle de la femme, tel qu'il existe et est tenu en fait - il sera déjà possible de déduire certaines valeurs, car tout rôle est un compromis entre les projets et la réalité, entre des attitudes (qui sont toujours liées à des valeurs) et la pression des conditions socio-économiques ${ }^{2}$.

Les recherches menées par le Centre de recherches sociologiques de l'Université de Vienne ont fourni une documentation sur les images qu'on se forme du rôle des femmes, grâce à l'étude de ce rôle tel qu'il est tenu en fait; dans la section ci-après, certains de ces résultats seront brièvement résumés. Dans la troisième et dernière partie du présent article, figureront une proposition concernant l'étude systématique des images qu'on se forme du rôle de la femme et quelques hypothèses relatives à l'évolution de certains aspects de ce rôle dans la société moderne, compte tenu de la continuité ou de la modification des images de ce rôle.

J. Leclercq et J. David, Die Familie, Zürich, 1955.

Par rôle, nous entendons un ensemble d'actions qui se répètent et la manière dont sont accomplies les tâches nécessaires au maintien et à la perpétuation d'un groupe social ou d'une institution dans une société. Les rôles sont tenus dans un cadre culturel prédéterminé, qui résulte à la fois de l'expérience qu'une société fait quotidiennement d'«idées directrices » et de «modèles d'action », et du degré d'attraction que conservent ces «modèles d'action ». Ce cadre n'a d'effet sur le plan social que si certaines conditions de caractère biologique, démographique et socio-économique sont remplies - autrement dit, seuls sont aptes à jouer certains rôles les gens qui sont d'un sexe et d'un âge déterminés et qui atteignent un certain niveau en matière d'éducation, de revenus et de biens. 


\section{Données relatives à la femme qui travaille et à la mère en ce qui concerne l'Autriche}

$\underline{\text { Retour à la table des matières de la femme autrichienne }}$

On trouvera, dans le tableau i, les données démographiques qui servent de base à l'étude du rôle des femmes en Autriche.

Tableau 1.

Répartition des femmes qui travaillent, par genre d'occupation, en Autriche (1).

Femmes exerçant une profession lucrative

$\begin{array}{cccc} & \text { Travaillant } & \text { Travaillant dans } & \text { Femme } \\ \text { Employées } & \text { à leur } & \text { une entreprise } & \text { n'exerçant pas } \\ \text { compte } & \text { familiale } & \text { de profession } \\ & \text { lucrative }\end{array}$

Ensemble des femmes (3 717 000)

Femmes âgées (2 308 000)

Femmes mariées (1 539 000)
20

31

13
5

6

4
11

19

16
54

44

67

1. Recensement de 1951 (Volkszählungsergebnisse, 1951). publié par l'Oesterreichisches Statistisches Zentralamt, Wien, 1953, Heft 14 (Text-band); et Hertha Firnberg, « Wesen und Wandel der Sozialschichtung 0esterreichs », dans : Oesterreichs Wirtschaft gestern, heute, morgen, publié sous la direction de Wilhelm, Weber, Berlin 1961, p. 125

Comme on le voit, 56\% des femmes âgées de 15 à 60 ans exerçaient, en 1951, une profession lucrative, et il est probable que cette proportion a beaucoup augmenté depuis lors. Mais près de la moitié de ces femmes travaillaient dans une entreprise familiale (exploitation agricole, entreprise artisanale ou petite affaire commerciale), soit pour leur propre compte, soit, plus souvent encore, pour aider leur mari, leur père, etc. Or les femmes qui entrent dans cette catégorie restent au sein de leur famille, même lorsqu'elles travaillent ; en général, le foyer et le lieu de travail sont, pour elles, beaucoup plus proches l'un de l'autre que ce n'est le cas pour la femme employée à l'extérieur. Le nombre des femmes travaillant dans ces conditions est bien plus élevé dans les régions rurales. Dans le tableau I, le pourcentage des femmes mariées exerçant une profession lucrative semble relativement faible, mais ce fait est dû, dans une large mesure, à la proportion particulièrement élevée de femmes âgées de plus de 60 ans en Autriche ${ }^{1}$. Ajoutons, pour clarifier notre problème central, que

1 Voir L. Rosemayr et E. Koeckeis, Leben und Wohnen alter Menschen in Heimstätten, Wien, 1960. 
les femmes représentaient, à l'époque considérée, 39\%, de la main-d'œuvre autrichienne.

Ces chiffres prouvent déjà qu'il existe en Autriche un problème général, relatif au rôle de la femme qui travaille, étant donné que, pour un très fort pourcentage de la population féminine, la vie quotidienne est marquée par un travail régulier accompli hors du foyer.

Depuis cinq ans, nous avons, mes collaborateurs et moi, mené in situ plusieurs enquêtes dont les résultats ont trait directement ou indirectement au rôle de la femme ${ }^{1}$. Nous pouvons résumer ces résultats de la manière suivante :

L'emploi des femmes correspond à une nécessité économique qui dépend de ce qu'on peut appeler le budget familial «de base ». Selon une étude effectuée à Vienne en 1955, les raisons financières qui incitent la femme à travailler sont évidemment en rapport avec le revenu du mari. Plus le gain du mari est élevé, moins les motifs économiques jouent de rôle dans la décision qui amène la femme à travailler à l'extérieur.

Sur la base de cette constatation, nous pouvons poser en principe que tout jugement porté par les femmes elles-mêmes sur leur travail montrerait que les motifs financiers qui les ont incitées à prendre un emploi l'emportent sur tous les autres.

L'étude de 3623 cas (hommes et femmes) nous a permis de comparer l'attitude des hommes et celle des femmes à l'égard de leur travail. Dans la mesure où cette étude a mis en lumière les motivations, nous avons constaté que le pourcentage des femmes qui exerçaient un emploi «parce qu'elles aimaient leur travail » était beaucoup plus faible que le pourcentage des hommes qui manifestaient de l' « enthousiasme » pour leur métier ${ }^{2}$.

La même étude a montré que le pourcentage des femmes qui déclaraient travailler pour des raisons autres que financières (intérêt pour leur travail, satisfaction personnelle, avantages sociaux) était très élevé dans l'enseignement, chez les artistes, dans les professions paramédicales, chez les assistantes sociales, etc., moyen chez les employées de bureau et le plus bas chez les travailleuses manuelles.

1 Wohnen dans Wien, Wien, 1965. L. Rosenmayr, «Die wiener Familie der Gegen-wart », in : Wiener Zeitschrift für Nervenheilkunde und deren Grenzgebiete, XIII /4, 1957, pp. 337-369. « The role of the woman and mother in the Viennese family of today », rapport présenté à la 5e réunion du stage d'études de l'Unesco consacré aux recherches sur la famille, Madrid, 1958. Der Wiener Geburtenrückgang im Lichte soziologischer Forschung, Internationaler Bevölkerungswissenschaftlicher Kongress, Wien, 1959 ; Wie lebt unsere Jugend? (en préparation). Ces documents décrivent les cas types et les méthodes de recherche qui ont permis d'obtenir les données mentionnées dans le présent article.

2 Il convient aussi d'interpréter ces résultats en tenant compte du fait que les femmes sont moins payées que les hommes pour un même travail ou pour un travail comparable. 
Tableau 2.

Motivation du travail.

\begin{tabular}{lcccc}
\hline \multicolumn{2}{c}{ Hommes } & \multicolumn{2}{c}{ Femmes } \\
& $\begin{array}{c}\text { Travaillant } \\
\text { à leur compte }\end{array}$ & Employés & $\begin{array}{c}\text { Travaillant } \\
\text { à leur compte }\end{array}$ & Employés \\
Raisons financières & 42 & 57 & 58 & 73 \\
Goût du travail effectué & 58 & 43 & 42 & 27 \\
& 100 & 100 & 100 & 100 \\
\hline
\end{tabular}

Ces résultats ont été précisés également, dans une large mesure, par une étude limitée à 240 entretiens psychanalytiques. Cette étude a montré que les mobiles financiers étaient mentionnés le plus souvent par les mères ayant trois enfants ou davantage. Cette constatation nous a permis de découvrir le conflit de fonctions suivant : plus le nombre des enfants est élevé, et plus pressante est la nécessité financière qui incite la mère à travailler à l'extérieur, alors que le besoin de sa présence au foyer se fait plus vivement sentir.

Il résultait clairement de notre enquête que la plupart des femmes (l'échantillon était de 240 sujets) étaient prêtes à quitter leur emploi au cas où leur mari le leur demanderait (59\% le quitteraient avec plaisir; $24 \%$ le quitteraient, mais préféreraient le garder ; $15 \%$ ne le quitteraient pas; 2 n'ont pas répondu).

Toutes ces données font nettement apparaître que, notamment dans les familles où le mari gagne peu ou qui comptent beaucoup d'enfants, le travail de l'épouse hors du foyer représente une extension du rôle qu'elle joue au sein de la famille.

Dans plusieurs études effectuées en Autriche, dans diverses régions et dans des classes sociales différentes, nous avons pu comparer le niveau d'instruction des maris et celui des épouses. Nous avons ainsi constaté ce qu'on peut appeler une «différence de niveau d'instruction entre époux ». Nous pouvons en déduire qu'il y a quelques dizaines d'années la majorité de la population devait admettre qu'en principe le rôle de la femme exigeait beaucoup moins d'instruction que celui de l'homme.

Notre étude ne nous a pas encore permis de déterminer comment l'homme conçoit l'activité de la femme sur le plan social. Cependant, nous constatons que, dans le domaine des relations amicales et sociales, l'homme exerce une influence dominante. Moins d'un quart seulement des relations sociales des ménages étudiés avaient été établies ou nouées par la femme. A Vienne, le choix des connaissances et des amis dépend surtout de l'homme, à la différence de ce qui se passe dans certaines classes de la société aux États-Unis, par exemple, où la femme exerce, en principe, une plus grande influence à cet égard - le très grand rôle joué par le voisinage dans la formation du réseau des relations sociales constituant, dans ce pays, un facteur qui est loin d'être négligeable. Naturellement, cette influence dominante de l'homme est peut-être due aux conceptions viennoises traditionnelles du rôle de la femme, selon lesquelles « le royaume de la femme est le foyer », alors que les relations à l'extérieur doivent être déterminées par le mari. Cette hypothèse se trouve confirmée par l'étude de l'histoire sociale de Vienne. Il y a seulement une cinquantaine d'années, il était encore 
tout à fait rare de voir une jeune fille ou une femme entrer seule (ou en compagnie d'une autre femme) dans un des cafés, alors fort populaires, de Vienne, bien que ces établissements ne fussent pas réservés aux hommes (comme le sont ou l'étaient les pubs anglais) : une femme n'y pénétrait jamais qu'en compagnie d'un homme. Jusqu'à la première guerre mondiale on a pu constater que les femmes qui appartenaient aux classes inférieures ou moyennes de la société viennoise ne prenaient qu'une part limitée aux distractions populaires; à cette époque fleurissaient les sociétés et les clubs d'hommes, où ceux-ci se rendaient le dimanche, tandis que la femme et les enfants restaient à la maison.

Il est intéressant de se faire une idée des valeurs qui s'attachent au rôle de la femme en analysant la culture populaire, telle qu'elle se manifeste dans le logement et l'ameublement, d'une part, et dans les activités récréatives, d'autre part. Plusieurs des enquêtes que nous avons faites à Vienne sur le logement confirment l'hypothèse selon laquelle certains éléments traditionnels de l'atmosphère agréable et de la Gemütlichkeit qu'apprécient tant les étrangers dans les distractions publiques de Vienne ne peuvent exister qu'au détriment du foyer et de certains aspects de la vie familiale. Dans ces conditions, le rôle de la femme perd l'appui de certains facteurs, et sa portée s'en trouve réduite. Nous pouvons en conclure que, si la femme, en tant qu'épouse et mère, est glorifiée à Vienne, on l'identifie moins au foyer qu'à la ville en tant que telle. La chanson Mei Muatterl war a Weanerin... (Ma mère était viennoise) où l'on trouve l'expression générale des idées qu'évoque la mère (de l'attachement du jeune homme tant à sa mère elle-même qu'à l'image qu'il s'en fait) se termine par la description d'une scène où la mère, du haut de l'une des collines qui entourent Vienne, montre la ville à son petit garçon. Ce qui est lié à l'image de la mère, c'est l'image de la ville, avec ses signes extérieurs de religion et de divertissement, plutôt que la réalité immédiate, plus sensible, de la Gestaltung culturelle et de l'influence de l'atmosphère du foyer.

Cependant, ce n'est pas seulement la mère qui évoque l'idée de la ville, c'est aussi la fille - ou les filles qui, comme le disent les chansons, personnifient le mieux le charme de la ville. La « cité des rêves » s'identifie aux "filles de rêve » dans la chanson populaire « Vienne, ma ville » (Wien, Wien, nur Du allein ... ). 


\section{Propositions et hypothèses en vue de nouvelles recherches}

$\underline{\text { Retour à la table des matières de la femme autrichienne }}$

Dans ce bref exposé des résultats de nos recherches, j'ai défini le type de travail féminin le plus répandu en Autriche comme étant « une extension du rôle que la femme joue au sein de la famille ». J'ai voulu indiquer par là que l'industrialisation, telle que la connaît actuellement la société autrichienne, a modifié la structure de l'emploi dans l'ensemble de la société (par exemple, la population agricole a diminué, la population industrielle a augmenté, les emplois de bureau sont plus nombreux, etc.) et qu'elle a aussi notablement modifié la structure de l'emploi féminin. La nature de ces changements (pour Vienne) apparaît nettement dans le tableau 3

Tableau 3.

Types d'emploi des femmes à Vienne (1).

\begin{tabular}{|c|c|c|c|c|c|c|}
\hline \multirow{2}{*}{\multicolumn{3}{|c|}{$\begin{array}{l}\text { Année et nombre total de } \\
\text { femmes }\end{array}$}} & \multirow{3}{*}{$\begin{array}{l}\text { Femmes travaillant } \\
\text { à leur compte } \\
20\end{array}$} & \multicolumn{3}{|c|}{ Femmes employées } \\
\hline & & & & \multirow{2}{*}{ Bureaux } & \multirow{2}{*}{$\begin{array}{l}\text { Travaux } \\
\text { manuels } \\
31\end{array}$} & \multirow{2}{*}{$\begin{array}{c}\begin{array}{c}\text { Travaux } \\
\text { domestiques }\end{array} \\
47\end{array}$} \\
\hline & 1880 & $(158501)$ & & & & \\
\hline & $1893(3)$ & (304 766) & 35 & 2 & 35 & 28 \\
\hline & 1900 & (365 70 & 39 & 4 & 30 & 27 \\
\hline & 1910 & $(478608$ & 42 & 7 & 30 & 21 \\
\hline & 1934 & (371 39 & 16 & 24 & 46 & 14 \\
\hline & 1951 & (329 511) & 14 & 33 & 47 & 6 \\
\hline \multicolumn{7}{|c|}{$\begin{array}{l}\text { (1) F. Olegnik, Historisch-Statistische Uebersichten von Wien, Mitteilungen aus Statistik und } \\
\text { Verwaltung der Stadt Wien, Sonderheft I, 1956, pp. 82, 84. }\end{array}$} \\
\hline \multicolumn{7}{|c|}{$\begin{array}{l}\text { (2) Y compris celles qui aident leur mari, leur père, etc., travaillant à son compte. } 3 \text {. Les changements } \\
\text { survenus de } 1880 \text { à } 1890 \text { sont principalement dus à une extension des limites administratives de la } \\
\text { zone métropolitaine. }\end{array}$} \\
\hline
\end{tabular}

En raison de ces changements et des besoins nouveaux de la société, il y a aujourd'hui moins de femmes qui travaillent dans des exploitations agricoles, dans des entreprises familiales (artisanales ou commerciales), ou comme bonnes ou domestiques dans les familles des classes supérieures. Cependant, celles qui travaillent hors du foyer le font encore, le plus souvent, dans l'intérêt de leur famille, tout comme autrefois ; naturellement, la vie familiale se modifie (certains travaux tels que 
la cuisine, l'aménagement du foyer, la couture et le raccommodage ne se font plus comme jadis); la famille nombreuse prend un caractère marginal, dans le cas des mères qui travaillent; on recherche de nouveaux types de coopération avec la génération précédente (parents, beaux-parents); il est probable aussi que certains aspects des relations de la femme avec son mari et ses enfants vont se transformer. Toutefois, les divers résultats de l'étude que nous avons consacrée à la sociologie de la jeunesse m'ont amené à penser que, si l'éducation est, en général, négligée, il ne faut pas en chercher la raison dans le travail de la mère hors du foyer.

Nous voudrions ajouter, comme corollaire à la thèse de «l'extension du rôle que joue la femme au sein de la famille », une autre observation générale, qu'il serait peutêtre intéressant d'étudier plus à fond : d'une part, la femme qui travaille hors du foyer le fait dans l'intérêt de la famille et, d'autre part, certaines fonctions sociales se modifient parce qu'elles sont exercées par des femmes. L'extension du rôle que joue la femme au sein de la famille a pour conséquence complémentaire que certaines fonctions sociales prennent un caractère maternel. Du fait qu'elles sont entrées dans le personnel enseignant, le corps médical, les services d'assistance sociale, les partis politiques et l'administration, les femmes ont modifié le caractère de certaines fonctions sociales ou, du moins, contribué à ouvrir de nouveaux horizons dans l'exercice de ces fonctions.

Nous ne pouvons nous étendre ici sur ce phénomène général, mais nous ne saurions terminer notre exposé sans signaler le conflit important - apparent ou réel ou même la contradiction qui existe entre les différents types actuels de la femme moderne. D'une part, les idées et les tendances favorables à la notion d'association entre égaux sont appuyées et encouragées (par l'enseignement mixte, les sports, l'emploi des femmes hors du foyer). D'autre part, le souci de distractions qui caractérise la civilisation moderne a fait des femmes l'élément principal de toutes les entreprises qui cherchent à stimuler l'intérêt du public et qui, en conséquence, tendent à séparer de bien des manières l'homme de la femme, afin d'exalter l'attrait de celle-ci. Les cover-girls, les vedettes qui vantent un produit, les mannequins, les hôtesses de l'air, les reines de beauté, les innombrables starlettes, enfin l'exploitation des sentiments qu'inspirent les «femmes malheureuses » (reines, princesses, etc.) - courante dans les magazines illustrés - tout cela met en valeur les caractéristiques de la femme et va à l'encontre de l'idée ou de la pratique de l' «association » et de la «camaraderie ». Il me semble que cette coexistence paradoxale de l'idée d'association, d'une part, et de l'exploitation de la féminité, d'autre part, représente une importante et significative constellation de rôles idéaux et de «modèles culturels » pour les femmes, qui appelle de nouvelles observations et de nouvelles études.

Paul Chombart de Lauwe a eu le mérite d'ouvrir un nouveau champ de recherches en proposant l'étude systématique de la conception que l'homme se fait du rôle de la femme. Il a également préconisé des recherches sur l'idée que se fait de son rôle la femme elle-même. Dans l'une des études que nous avons menées à Vienne, nous avons demandé à des femmes de comparer leur rôle avec les obligations et les tâches de leurs mères. Les résultats de cette enquête ont été très proches de ceux qu'avait obtenus Chombart à Paris ${ }^{1}$. De nouvelles recherches pourraient également donner des résultats intéressants, si l'on étudiait, par exemple, comment les parents envisagent l'avenir de leurs filles. Inversement, les images idéales de la mère que se forment les enfants ou les adolescents pourront faire l'objet d'une étude, en vue de laquelle l'un de

\footnotetext{
1 Voir, dans le présent numéro, l'article de P. Chombart de Lauwe, p. 7.
} 
nos projets de recherches sur les relations familiales des adolescents en Autriche fournira certaines données. D'autre part, une enquête sur ce que les adolescents attendent de leur futur mariage nous permettrait sans doute de tirer quelques conclusions sur les idées qu'ils se font, en général, du rôle de la femme.

Outre l'analyse des idées, des espoirs et des désirs des maris quant au rôle de la femme - éléments psychologiques qui sont tous fortement influencés par des facteurs subjectifs - il serait également souhaitable d'étudier la manière dont les institutions sociales conçoivent officiellement le rôle de la femme. Il y aurait donc intérêt à procéder à une enquête à ce sujet auprès des Églises, des syndicats, des partis politiques, des milieux d'affaires, etc. Enfin, il conviendrait d'interpréter les données ainsi obtenues à la lumière de l'histoire sociale et culturelle de la région considérée, car on rencontre souvent, notamment lorsqu'il s'agit de valeurs, les survivances d'anciennes traditions qui ne peuvent être comprises que dans leur contexte historique. Bien que cette façon d'opérer puisse encombrer la recherche d'un grand nombre de détails et risque de rendre les comparaisons encore plus difficiles sur le plan international, l'étude des valeurs bénéficiera, en fin de compte, des avantages d'une méthode à la fois complexe et exhaustive, et fournira ainsi au sociologue une base plus solide pour fonder ses jugements. 


\title{
VI \\ Les attitudes à l'égard de la condition de la femme en Yougoslavie
}

\author{
par Olivera Buric
}

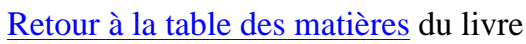

Table des matières de l'article sur la femme en Yougoslavie

1. Méthodologie de la recherche

a) Choix de l'échantillon

b) Technique de l'interview

c) Caractéristiques de l'échantillon

$\underline{\text { Retour à la table des matières de la femme yougoslave }}$

Dans le cadre d'une recherche ${ }^{1}$ organisée par l'Institut des sciences sociales de Belgrade, nous avons étudié, entre autres, les attitudes à l'égard du travail de la femme. L'étude de ce phénomène social nous a mis en contact avec un groupe de sociologues, qui sous la direction de P. H. Chombart de Lauwe, de l'École pratique des hautes études, de Paris, a mené une enquête internationale. Quoique notre participation ne fût pas prévue par le plan, l'analogie de nombreux éléments dans l'une et

Le thème de cette enquête est «L'influence du travail des femmes hors de leur domicile, sur la famille ». Elle a été effectuée aux mois de juin et de juillet 1960 et ses premiers résultats ont été présentés au vie Stage d'études international de la recherche sur la famille, qui a eu lieu en septembre 1960 en Yougoslavie. 
l'autre recherche permet d'inclure notre étude individuelle dans cette étude collective ${ }^{1}$.

Le travail de la femme est au centre de notre étude. Ce problème a été traité du point de vue de l'influence que l'industrialisation exerce sur les changements de la vie familiale, y compris les changements dans les attitudes à l'égard du travail de la femme. Nous avons entrepris de mesurer le processus de ces changements, car il était difficile d'effectuer une recherche empirique en vue de construire une image fixe du statut de la femme dans la société contemporaine. La société yougoslave se trouve soumise depuis vingt ans déjà a une évolution rapide. Le problème se complique encore du fait que le rythme de cette évolution varie suivant les région yougoslaves. En ce qui concerne notamment le statut de la femme, les attitudes sont intimement liées à l'arrière-plan ethnique, historique et religieux, qui est fort varié. C'est que, d'un côté, il existe des attitudes générales de la société concernant la question du statut de la femme -qui sont formulées dans une législation plus progressiste que beaucoup d'autres en vigueur dans des pays dont l'industrie est de loin plus développée que l'industrie yougoslave - tandis que, de l'autre côté, les mêmes individus qui professent, en principe, des attitudes aussi progressistes s'en tiennent, intimement, surtout lorsqu'il s'agit de leur propre femme, à une idéologie datant de l'ancienne société patriarcale.

Pourquoi nous sommes-nous arrêtés justement sur le travail de la femme comme facteur de la transformation des attitudes traditionnelles en attitudes modernes ? En premier lieu, parce que la place sans cesse croissante du travail féminin en Yougoslavie prouve qu'il s'agit là d'un processus social largement répandu. Mais la raison la plus importante, c'est que nous avons considéré la situation spécifique de la Yougoslavie comme particulièrement favorable à une telle analyse. En effet, au degré actuel de développement du pays, il est possible d'isoler le facteur «travail de la femme » et d'en évaluer l'influence sur les changements survenus dans les attitudes relatives à la condition de la femme dans la société. Nous ne croyons pas que la situation soit également favorable dans les autres pays où le processus de l'industrialisation s'est situé principalement avant l'inclusion en masse de la main-d'œuvre féminine dans l'économie et où les attitudes à l'égard du problème de la condition de la femme dans la société ont été modifiées sous l'influence de divers facteurs autres que l'industrialisation. En Yougoslavie, le processus de l'industrialisation se développe parallèlement au processus de l'inclusion rapide de la main-d'œuvre féminine dans l'économie, et l'idéologie patriarcale, en ce qui concerne les femmes, n'avait pas subi de changements sensibles avant l'intervention de ce dernier facteur qui, dans la plupart des cas, agit sur une population récemment arrivée d'un milieu rural à l'idéologie traditionnelle. Lorsque, dans une telle population, on compare les attitudes des femmes qui travaillent et celles de leurs maris avec les attitudes des femmes qui ne travaillent pas et celles de leurs maris, il devient possible d'isoler et de mesurer le rôle du facteur «travail des femmes » dans le changement des attitudes à l'égard de la condition de la femme dans la société. Cela nous a permis de formuler certaines hypothèses que le présent travail de recherche s'est attaché à vérifier.

Dans cet article, on se bornera à donner quelques détails sur les investigations touchant les changements dans l'idéologie de la famille ou, plus spécifiquement encore, les changements qui se sont produits dans les attitudes et les points de vue des

Antérieurement à l'enquête, des échanges de vues avaient eu lieu entre Mlle Kloskowska et M. P. H. Chombart de Lauwe sur certains problèmes de la recherche. 
femmes mariées et de leur mari en ce qui concerne le statut de la femme dans la société et la famille.

On a utilisé, dans les interviews des maris et des femmes, plusieurs espèces de questions, les unes se rapportant aux attitudes et opinions sur le statut général de la femme dans la famille, les autres aux attitudes et opinions des femmes concernant leurs propre travail, ou à celles des maris concernant le travail des épouses. À cette troisième catégorie appartient une question projective que l'on posait au mari et qui intéressait l'attitude du milieu en ce qui concerne le travail des femmes en général. À titre d'illustration, nous citerons certaines de ces questions. La quinzième ${ }^{1}$, par exemple, comprend 9 points touchant les rôles fondamentaux que la femme doit jouer dans la famille. À la femme interrogée on précisait toujours qu'elle devait répondre compte tenu des rôles qu'elle estimait souhaitables d'une façon générale et non pas des rôles qu'elle jouait réellement dans sa famille. De l'ensemble de ces réponses on déduit comment les femmes mariées se représentent la condition d'une épouse-mère dans la famille. Ces points étaient approximativement conçus en ces termes :

1. L'éducation des enfants incombe à la mère et non au père.

2. La femme doit faire la cuisine et nettoyer la maison, tandis que le mari doit gagner l'argent de la famille.

3. Il est honteux pour un mari d'aider sa femme dans les travaux domestiques (ménage et cuisine).

4. Lorsque le mari rentre de son travail, il est absolument nécessaire de lui créer des conditions favorables au repos, sans égard à la situation qui règne dans la maison.

5. Si la femme a des enfants, sa place est au foyer et elle ne doit pas travailler audehors.

6. Il est contraire à la nature humaine que la femme jouisse d'une autorité égale à celle de l'homme, soit à son poste de travail, soit à la maison.

7. La femme doit laisser son mari s'occuper de la politique et des questions sociales, car il est plus versé qu'elle en ces domaines.

8. Le mari qui passe ses loisirs avec la femme est un «pantouflard ». Il doit sortir avec d'autres hommes.

9. C'est le mari qui doit prendre les décisions importantes dans la famille.

Les définitions des rôles respectifs de la femme et du mari ont été formulées d'une façon négative, afin d'éviter de suggestionner les sujets interrogés. Dans les réponses à ces questions, on a prévu cinq degrés, selon Likert, depuis l'approbation totale et l'absence de toute attitude définie,jusqu'au refus complet. Suivant les réponses, un classement des femmes est établi allant de celles qui possèdent une idéologie de famille fort traditionnelle à celles qui ont atteint le plus haut degré d'émancipation. Comme nous l'avons déjà souligné, nous ne sommes pas en mesure d'exposer les résultats définitifs, car les recherches se poursuivent, mais, à en juger d'après l'expérience acquise jusqu'ici, cette question promet d'être fructueuse.

1 Le Dr Liros Hoffman de l'Université d'Ann Arbor, Michigan (États-Unis), avait posé une question analogue lors d'une enquête effectuée sur un échantillon de la population américaine. Dans notre étude, la question posée diffère de celle du Dr Liros en ce qu'elle a été adaptée à la situation nationale. Elle n'a pas, dans le cadre de l'étude entière, la même fonction de contrôle. Elle vérifie pas, néanmoins nos hypothèses relatives aux causes des attitudes. 
Les questions 26 à 33 permettent de demander aux femmes qui travaillent ainsi qu'aux ménagères ce qu'elles pensent de leur emploi ou de leur inoccupation, quels sont les avantages et les défauts du statut de la femme qui travaille et de celui de la femme-ménagère. Aux unes on demande encore si elles désirent obtenir un emploi et, si oui, ce qui constitue à leurs yeux le plus grand obstacle à cet égard. Aux autres on demande si elles désirent conserver leur emploi ou le quitter.

Dans un questionnaire destiné spécialement aux maris, on demande à ceux-ci ce qu'ils pensent de l'emploi des femmes en général, s'ils l'approuvent ou le désapprouvent, ce qu'ils pensent de l'emploi de leur propre femme, s'ils désirent qu'elle le quitte ou, dans le cas où elle n'en a pas, qu'elle en obtienne un. On les invite à préciser les motifs de leurs attitudes. On demande également aux maris s'ils pensent que les gens avec lesquels ils sont en contact (collègues, concitoyens, voisins, parents, etc.) préfèrent voir les femmes travailler hors du domicile conjugal ou ne pas travailler.

Il existe une autre question ${ }^{1}$ que l'on pose sous une forme identique aux deux époux et qui est conçue en ces termes : " Si votre femme a pris un emploi au cours de votre vie conjugale, quels changements se sont produits de ce fait dans votre famille ? » Les réponses à cette question sont rares, car elle intéresse seulement parmi les femmes qui travaillent celles qui ont pris un emploi depuis leur mariage, ainsi que leurs maris. Or la plupart des femmes travaillant hors de chez elles ont pris leur emploi avant de se marier.

Toutes ces questions ont pour objet de vérifier des données spécifiques qui se rapportent à l'élaboration d'une hypothèse fondamentale sur l'idéologie de la famille.

On suppose, tout d'abord, que le facteur «travail des femmes » agira de telle sorte que les femmes employées seront d'avis, plus souvent que les femmes sans emploi, que l'éducation des enfants n'est pas exclusivement du ressort de la mère. On suppose, parallèlement, que ces femmes estimeront que leur rôle dans les tâches quotidiennes du ménage doit être allégé au détriment du mari, tandis que leur rôle dans la prise des décisions importantes touchant la vie familiale et sociale doit augmenter. Ces attitudes générales doivent se refléter non seulement sur les attitudes concernant l'emploi personnel, mais aussi sur la situation réelle dans la maison. On suppose aussi que les femmes de cette catégorie considéreront que leur emploi produit plus d'effets positifs que négatifs sur la famille, particulièrement sur le plan matériel, et qu'elles ne seront pas prêtes à le quitter, mais désireront plutôt le garder. On suppose que le rapport entre les attitudes générales et personnelles sera également harmonieux chez les maris. On s'attend que ceux-ci projettent leurs attitudes également sur leur entourage et l'on pense que la plupart des gens avec lesquels ils sont en contact seront favorables à l'emploi de la femme. On présume qu'on mentionnera comme principaux désavantages de l'emploi des femmes les conditions peu satisfaisantes de l'éducation des enfants en l'absence de la mère et la négligence à l'égard de la maison et des travaux domestiques.

L'insertion de cette question m'a été suggérée par le Dr Viola Klein, de la London School of Economics, de Londres. Elle sert en fait à vérifier les résultats d'une enquête que le Dr Klein a déjà effectuée sur une population anglaise, en n'interrogeant que les maris des femmes qui travaillent hors du domicile conjugal. (Voir: Viola Klein, Working wives, Institute of Personnel Management, 80 Fetter Lane, London W.C.4. ) 
Chez les femmes qui n'ont pas d'emploi hors de leur foyer, aussi bien que chez leurs maris, on s'attend que les attitudes concernant l'emploi des femmes en général, ainsi que leur propre statut de travail, soient plus discordantes que dans les familles où la femme a un emploi. Les femmes et les maris déclareront sans doute que les femmes devraient en principe exercer un emploi, tout en faisant savoir que, dans la pratique, ils ne tiennent pas à ce que les femmes travaillent. On présume que la question projective concernant l'opinion de l'entourage sur l'emploi des femmes s'accordera, comme ce fut le cas des maris dont les femmes travaillent, avec les attitudes personnelles concernant cette question.

Il est intéressant de mentionner, d'après des données encore incomplètes, que ce sont les maris, dans les deux groupes de familles, qui se plaignent du travail de la femme, plutôt que les femmes elles-mêmes. Si la femme exerce un emploi, elle veut généralement le garder; elle n'attend aucune aide de la part du mari dans les travaux du ménage et ne se plaint jamais :elle compte plutôt être secourue par la société. Si elle n'est pas employée,elle désire, dans la plupart des cas, le devenir. Les principaux obstacles auxquels se heurte la réalisation de ce désir sont, d'une part, sa préoccupation à l'égard des enfants et, d'autre part, le manque de qualifications.

Outre ces questions et hypothèses qui se rapportent aux attitudes, il existe un nombre considérable de questions qui se rapportent à la situation concrète dans la maison. Entre autres, elles doivent permettre d'établir si le statut de la femme dans la famille est en harmonie avec les attitudes générales et personnelles des époux. Après les hypothèses formulées à ce sujet, on s'attend que, dans la famille où la femme travaille hors du domicile conjugal, les attitudes générales du mari et de la femme et la situation concrète dans la maison seront plus en harmonie que dans les familles où la femme, ne travaillant pas hors de chez elle, s'occupe seulement du travail ménager. A en juger d'après les premières analyses, cette hypothèse s'est révélée juste. Les femmes travaillant hors du domicile conjugal ont groupé leurs réponses à la question no 15 , mentionnée ci-dessus, dans la partie qui est opposée à l'idéologie patriarcale, c'est-à-dire que leurs attitudes touchant la situation du mari dans la famille reflètent une idéologie égalitaire des rapports entre mari et femme. Les autres questions ont permis d'établir que la situation de ces mêmes femmes dans leur famille est effectivement caractérisée par une plus grande égalité de droits et par davantage d'autorité. On le sent particulièrement dans les réponses du mari à la question "Que faites-vous lorsque votre femme vous agace ? », lorsqu'il choisit, entre 9 points possibles, ceux qui montrent qu'il essaie de se contrôler, même s'il est en colère, et qu'il se permet de moins en moins d'être grossier dans ses querelles avec sa femme. Par contre, les femmes qui ne travaillent pas hors de chez elles attribuent une situation dominante à leur mari, dans la famille, et bénéficient, en même temps, d'une moindre autorité et de moins de respect de la part de leur mari, qui se conduit plus grossièrement avec elles.

Nous ne serons pas en mesure de donner des résultats précis ni de tirer des conclusions des hypothèses formulées avant que toutes les données recueillies aient été analysées. 


\section{Méthodologie de la recherche}

La méthode employée s'appuie sur une étude en profondeur et son caractère est avant tout explicatif. L'enquête n'est pas extensive, elle porte sur une assez petite population et vise à dégager le rapport causal existant entre le travail des femmes et les changements survenus dans la vie et l'idéologie de la famille. Comme instrument de mesure, on a utilisé l'expérimentation ex post facto. Elle s'est effectuée en une seule période avec comparaison de deux groupes de familles : les familles où la femme travaille hors du domicile conjugal représentent « le groupe expérimental » et les familles où la femme ne travaille pas hors du domicile conjugal, c'est-à-dire où elle s'occupe uniquement des travaux du ménage, "le groupe de contrôle ». La comparaison entre ces deux groupes permet d'apprécier l'effet du facteur « travail de la femme ».

Afin d'éviter les défauts inhérents à ce genre de recherches (impossibilité de mesurer avant et après l'effet du facteur), nous avons introduit, autant que possible, des éléments de contrôle, non seulement dans le choix de l'échantillon, mais aussi dans l'accomplissement des analyses.

\section{a) Choix de l'échantillon}

Retour à la table des matières de la femme yougoslave

Divers critères qui avaient pour but d'obtenir une population autant que possible homogène afin de permettre la comparaison expérimentale ont présidé au choix de l'échantillon:

1. Niveau socio-économique : toutes les familles appartenaient à la classe ouvrière et avaient des revenus comparables.

2. Résidence : toutes les familles avaient les mêmes conditions de logement, elles occupaient des habitations allouées par les entreprises dans les diverses banlieues de Belgrade.

3. Présence d'enfants : toutes les familles avaient au moins un enfant âgé de moins de 15 ans.

4. Origine ethnique : tous les sujets interrogés appartenaient à la nationalité serbe. 
5. Toutes les familles étaient des familles complètes, c'est-à-dire qu'au moment de l'enquête le mari, la femme et les enfants vivaient ensemble.

Les familles ainsi choisies étaient interrogées deux par deux, selon le statut du travail de la femme, les qualifications du mari et le nombre et l'âge des enfants.

\section{b) Technique de l'interview}

L'enquête a été menée dans les logements des ouvriers. Le principal interviewé était l'épouse. L'enquête durait environ une heure et demie. Tandis qu'on interrogeait la femme, un enquêteur expérimenté remettait au mari, si celui-ci se trouvait chez lui à ce moment-là, un questionnaire spécial, plus court, qu'il devait remplir tout seul, dans la pièce contiguë, pour éviter que les époux ne s'influencent réciproquement. Mais les résultats de cette interview ne couvrent qu'un sous-échantillonnage, qui se faisait au hasard, car l'enquête se limitait aux maris présents à la maison au moment où l'on interrogeait la femme.

\section{c) Caractéristiques de l'échantillon}

$\underline{\text { Retour à la table des matières de la femme yougoslave }}$

L'échantillon comprenait 206 familles (206 femmes et 120 maris, soit 326 personnes en tout).

Des premières analyses effectuées, on peut conclure que l'homogénéité des deux groupes a été réalisée à un très haut degré. D'abord, ils sont tout à fait identiques par le nombre des interviewés : 103 femmes et 60 maris dans chacun. Cette homogénéité a été obtenue également en ce qui concerne les caractéristiques suivantes :

Appartenance sociale (mesurée selon l'occupation et l'éducation du mari).

1. Occupation du mari : dans la totalité des 206 familles, les maris sont des travailleurs manuels qui se répartissent ainsi : ouvriers spécialisés, 41,7\% ouvriers qualifiés, $49,5 \%$ ouvriers semi-qualifiés ${ }^{1} 8,8 \%$ Leur répartition dans les groupes d'expérimentation était complètement identique.

2. Pourcentage des familles selon l'éducation du mari (quatre années d'école primaire, $15,6 \%$ quatre années d'école secondaire, $8,7 \%$; école professionnelle formant des ouvriers qualifiés, 47,6\% école professionnelle formant des ouvriers spécialisés, $28,1 \%$. Leur répartition entre les deux groupes d'expérimentation était presque identique. Dans chacun, le degré le plus bas de l'éducation du mari correspondait à quatre années d'école primaire et le degré le plus élevé à l'école professionnelle formant des ouvriers spécialisés.

1 Le très petit nombre d'ouvriers semi-qualifiés et l'absence totale d'ouvriers non qualifiés sont dus au fait que ces catégories comprennent essentiellement des jeunes gens célibataires et sans enfants. 
Origine sociale (mesurée selon l'appartenance sociale du père du mari).

1. Occupation du père (paysan, 66,9\% autre occupation, 33,1\%). La répartition des sujets entre les deux groupes d'expérimentation est presque identique (prédominance de l'élément paysan).

2. Éducation du père (aucune instruction scolaire, 26,7\% ; quatre années d'école primaire, 54,4\% ; autres écoles et inconnu, 18,9\%. La répartition entre les deux groupes d'expérimentation est presque identique (pourcentage très élevé de pères n'ayant reçu aucune éducation ou une éducation rudimentaire à l'école primaire).

Présence d'enfants. Nous avons bien pris soin d'assurer une répartition régulière des enfants dans l'un et l'autre type de famille et nous avons obtenu une homogénéité complète.

1. Pourcentage des familles comptant 1,2 et 3 enfants ${ }^{1}$ (1 enfant, 44,7; 2 enfants, 43,7\%; 3 enfants, 11,6\%).

2. Pourcentage d'enfants dans les familles ( 1 enfant, 26,7\%; 2 enfants, $52,4 \% ; 3$ enfants, 20,9\%).

3. Pourcentage des familles d'après l'âge du plus jeune enfants ${ }^{2}$ (jusqu'à 2 ans, $21,3 \%$; de 3 à 5 ans, 21,3\% de 6 à 10 ans, 44,7\%; de 11 à 14 ans, 12,7\%; de 15 ans et plus ${ }^{3}$.

4. Nombre d'enfants selon l'âge (jusqu'à 2 ans, $12,8 \%$ de 3 à 5 ans, 17,5\%; de 6 à 10 ans, 43,6\%; de 11 à 14 ans, 21,85 de 15 ans et plus, 4,3\%). Les enfants sont répartis par âge presque identiquement dans les deux groupes d'expérimentation. Une petite différence apparaît seulement dans le groupe d'enfants âgés de plus de 6 ans.

5. Nombre d'enfants selon les qualifications du père (ouvrier spécialisé, $43 \%$ ouvrier qualifié, 46,7\% ; ouvrier semi-qualifié, 10,3\%). La répartition est tout à fait identique dans les deux groupes.

On a réussi ainsi à réaliser une répartition presque identique selon le nombre d'enfants, leur âge et les qualifications du père.

Âge des époux. Il se situe presque toujours entre 20 et 45 ans. La répartition est aussi presque identique dans les deux types de famille (familles où la femme travaille : femme, $98 \%$; mari, $94,2 \%$; familles où la femme ne travaille pas : femme, $99 \%$ mari, $90,3 \%$ )

1 Les familles comptant plus de 3 enfants étaient faiblement représentées et, par conséquent, tenues pour insignifiantes du point de vue statistique, elles étaient laissées à l'écart de l'échantillon.

2 Pour la classification des familles comptant un enfant, nous avons pris l'âge de cet enfant unique, tandis que, pour la classification des familles de plusieurs enfants, nous avons pris l'âge du plus jeune. Nous avons agi ainsi parce que nous sommes d'avis que le cycle vital d'une famille est déterminé par l'âge du plus jeune des enfants.

3 L'enquête comprend également des enfants âgés de plus de 15 ans, mais, conformément aux critères adoptés, dans aucune famille cet enfant n'est le plus jeune. 
Si un échantillon choisi de cette façon présente des avantages, il n'est pas plus exempt d'inconvénients que les investigations analogues sur les autres groupes socioéconomiques et ethniques de Yougoslavie. Nous espérons que telle quelle l'enquête permettra d'étendre les résultats de la recherche à une plus vaste population et d'obtenir ainsi des généralisations d'une validité supérieure.

S'il est possible d'établir les comparaisons sur un plan encore plus vaste, en l'occurrence sur le plan international, certaines généralisations pourront s'affirmer davantage. Nous sommes heureux d'avoir pu participer à un travail de ce genre et nous espérons qu'une fois terminées nos analyses seront utiles au groupe de sociologues qui ont entrepris la tâche difficile d'examiner comment les hommes perçoivent et se représentent la société et l'évolution des structures sociales.

Fin du texte 